\title{
Enquêter, former, publier au cœur de la cité
}

Sous la direction de Monica Battaglini, Stéphanie Fretz, Eva Nada et Laurence Ossipow Préface de Monique Eckmann

ies éditions

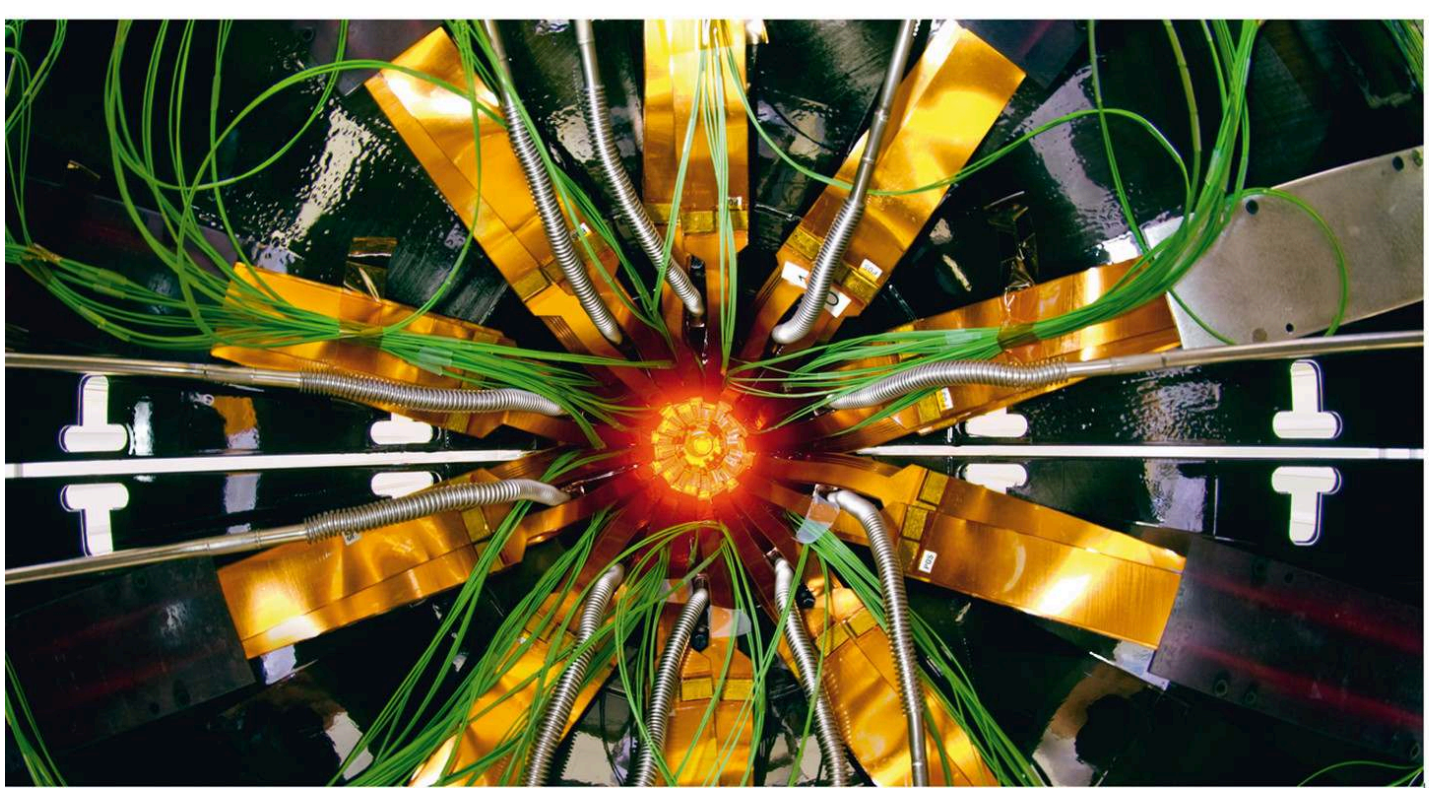




\section{Enquêter, former, publier au cœur de la cité}

Monica Battaglini, Stéphanie Fretz, Eva Nada et Laurence Ossipow (dir.)

DOI : 10.4000/books.ies.2101

Éditeur : Éditions ies

Année d'édition : 2018

Date de mise en ligne : 18 février 2019

Collection : Le social dans la cité

ISBN électronique : 9782882241368

\section{QboOoks}

http://books.openedition.org

\section{Édition imprimée}

ISBN : 9782882242006

Nombre de pages : 256

\section{Référence électronique}

BATTAGLINI, Monica (dir.) ; et al. Enquêter, former, publier au cœur de la cité. Nouvelle édition [en ligne]. Genève : Éditions ies, 2018 (généré le 01 février 2021). Disponible sur Internet : <http:// books.openedition.org/ies/2101>. ISBN : 9782882241368. DOI : https://doi.org/10.4000/books.ies. 2101. 
Cet ouvrage est diffusé en accès ouvert dans le cadre du projet OpenEdition Books Select.

Ce programme de financement participatif, coordonné par OpenEdition en partenariat avec Knowledge Unlatched et le consortium Couperin, permet aux bibliothèques de contribuer à la libération de contenus provenant d'éditeurs majeurs dans le domaine des sciences humaines et sociales. La liste des bibliothèques ayant contribué financièrement à la libération de cet ouvrage se trouve ici :

https://www.openedition.org/22515.

This book is published open access as part of the OpenEdition Books Select project.

This crowdfunding program is coordinated by OpenEdition in partnership with Knowledge Unlatched and the French library consortium Couperin. Thanks to the initiative, libraries can contribute to unlatch content from key publishers in the Humanities and Social Sciences. Discover all the libraries that helped to make this book available open access:

https://www.openedition.org/22515?lang=en.

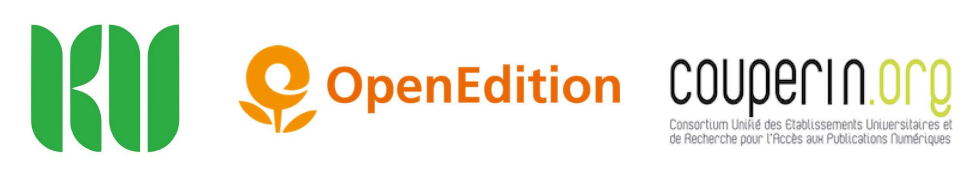


Enquêter, former, publier au cœur de la cité 


\section{Le Centre de recherche sociale}

Depuis sa création en I969, le Centre de recherche sociale (CERES) mène des enquêtes sur des questions de société - en lien avec les notions de cohésion, de norme, de déviance, de diversité, d'inégalité, de vulnérabilité, etc. - interrogeant et donnant à voir que la relation entre l'action sociale et ses publics cibles demeure marquée et travaillée par les contextes dans lesquels elle se situe et qu'elle contribue à transformer.

Par la déconstruction de certaines évidences, la mise en lumière de réalités cachées, l'explicitation de normativités, l'analyse d'ambivalences, la restitution et la mise en valeur de savoir-faire..., les connaissances produites par le CERES entendent contribuer à une analyse approfondie de réalités relatives aux politiques sociales, à l'intervention sociale et aux populations concernées par ces premières.

Cette démarche réflexive de recherche s'inscrit dans le cadre d'une collaboration étroite avec les milieux institutionnels, professionnels ou les publics intéressés. Elle souhaite concourir à la reconnaissance et au renforcement des capacités critiques, créatrices et innovantes à l'œuvre dans l'action sociale, et à consolider l'expertise déployée par les champs professionnels.

\section{h $\quad$ e $\quad t \quad s$}

Haute école de travail social

Genève

Centre de recherche

sociale (CERES) 
le social dans la cité 27

Enquêter, former, publier au cœur de la cité

Sous la direction de

Monica Battaglini, Stéphanie Fretz,

Eva Nada et Laurence Ossipow

Préface de

Monique Eckmann 
Ouvrage publié avec le soutien de la Haute école spécialisée de Suisse occidentale (HES-SO)

\section{Hes.so}

Haute Ecole Spécialisée
de Suisse occidentale Fachhochschule Westschweiz University of Applied Sciences and Arts
Western Switzerland

Responsable de collection: Monica Battaglini

Révision linguistique: Alexandra Rihs

Mise en page: Gaia Biaggi et Marc Logoz

Couverture: Jean-Marc Humm

Impression et reliure: Prestige graphique

() 2018 Editions ies

Tous droits de traduction, d'adaptation et de reproduction d'un extrait quelconque de ce livre, par quelque procédé que ce soit, réservés pour tous les pays.

ISBN: 978-2-88224-200-6

ISSN: 1663-9499

Dépôt légal: octobre 2018

Editions ies

Haute école de travail social, Genève

editions.hets@hesge.ch

www.hesge.ch/hets/editions-ies 


\section{Sommaire}

7 Préface

Monique Eckmann

15 Introduction

Monica Battaglini, Stéphanie Fretz, Eva Nada et Laurence Ossipow

Première partie

La recherche dans la cité

27 Quels liens entre recherche et travail social?

Claudio Bolzman

45 La recherche dans les hautes écoles, entre "débat académique" et "débat citoyen" Monica Battaglini et Laurent Wicht

67 Enquêter en «terrain difficile» Arnaud Frauenfelder, Géraldine Bugnon et Eva Nada

89 Posture du ou de la chercheur.e dans la relation d'entretien avec des jeunes ayant des parcours migratoires difficiles Théogène-Octave Gakuba

Deuxième partie

Recherche et enseignement

111 La recherche anthropologique dans l'enseignement en travail social Laurence Ossipow 
131 La posture professionnelle et la réflexivité en travail social envisagées sous l'angle ethnographique et esthétique Francis Loser

151 Faire se rencontrer recherche, intervention et formation Sylvie Mezzena et Kim Stroumza

173 L'imbrication heuristique entre terrain, recherche et enseignement

Sophie Rodari et Laurence Bachmann

193 Prévenir la maltraitance envers les personnes vulnérables Manon Masse

Troisième partie

Et au-delà

219 A la recherche du livre perdu Stéphanie Fretz

237 Recherche en contexteS Postface d'Eva Nada

253 Présentation des contributrices et contributeurs 


\section{Préface}

\section{Monique Eckmann}

Les chercheur.e.s sont incité.e.s à fonctionner au sein d'un circuit qui se déploie ainsi: dépôt de requête pour recherche de fonds (si fonds trouvés), récolte de données, transcription ou description des données, analyse, puis rédaction de rapports ou autre publication, tout en effectuant de concert la prochaine requête pour obtenir de nouveaux fonds.

Ainsi, les questions fondamentales que soulève cet ouvrage restent souvent en arrière-plan de ces préoccupations, voire même impensées dans le feu de l'action, alors qu'elles traversent de manière fondamentale chacune des recherches menées à la Haute école de travail social (HETS), et au-delà.

Ayant travaillé pendant de nombreuses années à la HETS comme professeure et chercheure, et ayant dirigé les Editions ies, j'ai été moi-même prise dans le circuit. Je livre ici quelques points ressortis de ma lecture des contributions de mes collègues, points qui me semblent constituer des enjeux particuliers pour la recherche et le développement (Ra\&D) dans une haute école spécialisée.

Parcourons donc quelques-uns de ces enjeux et questions soulevés, à savoir la question de la genèse des recherches et de la circularité entre formation, prestations de service et recherche, la place des diverses actrices et acteurs dans les dispositifs de recherche, les relations de pouvoir auxquelles les chercheur·e·s sont confronté·e·s et, enfin, la restitution de la recherche et ce qu'elle peut amener à la cité.

\section{Genèse des recherches et circularité}

La première dimension qui frappe est la diversité des origines et des genèses des recherches initiées à la HETS. 
D'un côté, une grande partie des recherches prennent leur source dans la connaissance des terrains professionnels, des problématiques des usagères et usagers, des professionnel.le.s ou des politiques sociales et éducatives. Si l'on peut le formuler ainsi, la «chance» des HES est leur lien étroit avec les terrains professionnels, qui se concrétise notamment par l'accompagnement de formations pratiques des étudiant·e•s, par les analyses de situations amenées par ces derniers durant les études, que ce soit en formation de base, durant le master ou en formation continue. Ainsi, les chercheur·e.s-enseignant.e.s sont sans cesse confronté.e.s à l'actualisation des problèmes tels qu'ils sont vécus par les partenaires des milieux du travail social. Ce sont donc les observations des travailleuses et travailleurs sociaux, les difficultés et obstacles auxquels ils et elles sont confronté.e.s qui sont une des sources premières des projets de recherche. A cela s'ajoute que les institutions et associations partenaires, tout comme les pouvoirs publics, peuvent se constituer en demandeuses et demandeurs d'études, mettant au concours des mandats de recherche. Ces demandes sont également issues d'interrogations concernant l'exercice des professions sociales, ou encore des questions de politiques publiques.

De l'autre côté, on trouve, comme dans toute institution académique, des recherches en lien avec des objets spécifiques aux chercheur·e•s, basées sur leurs travaux précédents, liées à leurs questionnements théoriques, leurs interrogations pratiques ou leurs outils méthodologiques.

Les opportunités que procurent les appels d'offre de type PNR, lancés par le Fonds national de la recherche scientifique suisse par exemple, optimisent la possibilité de combiner ces deux types de genèses de projets de recherches.

Ainsi, la recherche s'inscrit-elle dans une circularité où formation, recherche, formation continue et prestations de service s'alimentent mutuellement. Une circularité qu'on peut schématiser ainsi:

Contacts par la formation avec les milieux professionnels $\rightarrow$ problématisation $\rightarrow$ requêtes auprès de fonds de recherche/mandats publics ou privés $\rightarrow$ publications et autres valorisations $\rightarrow$ enseignement en formation de base et continue; prestations de service $\rightarrow$ nouvelles questions et nouvelles requêtes ou mandats de recherche, etc.

Si cette situation constitue indéniablement, à mes yeux, une opportunité tant pour la recherche que pour la formation, cela nécessite cependant que les divers secteurs impliqués dans cette circularité se tiennent 
au courant réciproquement et cultivent un dialogue nourri au sujet des questions théoriques et pratiques, des méthodologies et des résultats des recherches. Cela nécessite de rapprocher non seulement formation de base et recherche, mais aussi formation continue et prestations de service et recherche, sans négliger le service de publication des Editions ies, qui constitue un maillon de diffusion crucial dans cette circularité.

\section{Dispositif de recherche et place des actrices et acteurs impliqué.e.s}

Une deuxième dimension qui peut être mentionnée est celle du dispositif de recherche. Un dispositif de recherche inclut non seulement des méthodes de récolte de données et des méthodes d'analyse de ces dernières, mais également l'agencement des places occupées par les divers partenaires, ainsi que les fonctions qui leurs sont assignées en lien avec des droits et des devoirs. Et les actrices et acteurs sont souvent nombreux: responsables institutionnel.le.s - tant au niveau des directions qu'au niveau des responsables d'équipe -, professionnel.le.s - parfois de divers secteurs, animatrices et animateurs, enseignant.e.s, soignant.e.s, ou psychologues, assistantes et assistants sociaux et éducatrices et éducateurs, etc. -, usagères et usagers ou bénéficiaires; et parfois encore, les familles ou autres proches. Le défi est d'expliciter et de définir de quelle façon ces divers acteurs et actrices sont inclus·e.s dans le processus, et ce que l'équipe de recherche leur propose en arrivant sur «leur» terrain: est-ce un public uniquement sollicité pour fournir des informations? Est-il observé ou observateur? Est-il objet de recherche ou co-chercheur? A-t-il un droit d'interprétation des données et quel est son droit de regard sur les conclusions? Il s'agit donc de négocier et de définir les modalités de collaboration et les droits et devoirs des un.e.s et des autres.

Les professionnel-le.s occupent à cet égard une position-clé dans ces dispositifs car, souvent, leur savoir-faire, leur expérience de terrain et leurs observations sont indispensables aux chercheur.e.s. Leurs observations peuvent avoir des biais, des angles morts ou des zones d'opacité liées au souci de préserver les intérêts des usagères et usagers ou encore leur propre statut, mais cela fait précisément partie de la recherche que de valoriser leurs savoirs et leurs observations, tout en étant en mesure de les questionner, d'en discuter et d'élaborer une réflexion. 
Cela renvoie également à la place et à la posture des chercheur.e.s dans leur apparente neutralité. Or, en sciences humaines, la chercheure ou le chercheur n'est jamais en dehors de son objet, elle ou il fait partie de celui-ci comme membre de la société, avec ses propres ambivalences, ses attaches, ses distances; elle ou il a un positionnement, ne serait-ce que de façon implicite. Ainsi, cela ferait en principe partie de la réflexion sur le dispositif que de clarifier, d'expliciter la position de la chercheure ou du chercheur par rapport à son objet, de mettre à plat ses rapports d'intériorité ou d'extériorité, d'appartenance et de distance, de même que les outils auxquels elle ou il a recours pour garantir une certaine neutralité. Expliciter en quoi la recherche affecte et transforme non seulement le terrain, mais en quoi elle affecte et transforme aussi les chercheur.e.s pourrait être bénéfique.

Ces réflexions nous amènent aux relations de pouvoir qui se jouent de manière inhérente au cours d'une recherche.

\section{Les relations de pouvoir}

La recherche demande une gestion délicate des relations de pouvoir entre chercheur.e.s et milieux professionnels, qui se manifestent selon au moins trois dimensions: les relations entre organisme mandant et mandataires, les relations entre chercheur.e.s et professionnel.le·s, et les relations entre chercheur.e.s et usagères ou usagers.

La complexité des relations entre mandant·e.s et mandataires a été démontrée dans plusieurs contributions de ce volume, et il s'avère souvent nécessaire de renégocier le mandat au cours de l'élaboration du contrat, car les attentes et les représentations des mandant.e.s ne correspondent pas toujours aux possibilités réalistes de la recherche. Aussi, la définition du "problème» tel qu'il est identifié par les mandant.e.s doit parfois être remaniée. Ces négociations et remodelage font partie intégrante du processus de recherche et permettent d'arriver à une meilleure entente entre mandant.e.s et mandataires. Il est toutefois possible que cela engendre des tensions importantes qui rendent difficile la poursuite de la collaboration.

Une deuxième dimension des relations de pouvoir, déjà évoquée, est formée par les rapports entre chercheur.e.s et professionnel.le.s. Les professionnel.le·s fournissent souvent la matière première pour la recherche, notamment lorsque les pratiques professionnelles se trouvent au cœur 
même de l'observation: les échanges et contacts entre professionnel-le·s, usagères et usagers, les modalités d'intervention, leurs analyses des problématiques amenées par les usagères et usagers, leurs visions de la politique sociale ou d'immigration, etc. En livrant ainsi aux chercheur.e.s l'intimité de leur travail quotidien, leurs questionnements et leurs doutes, leurs expériences et compétences, elles et ils livrent de fait à la recherche ses matériaux de base. Or, le statut des professionnel-le·s dans le dispositif de recherche est souvent ambigu, oscillant entre objet d'étude et partenaire. Quel est leur statut? Quelle est leur rémunération matérielle? Mais aussi symbolique, de par sa place dans la discussion et la publication des méthodes et résultats de recherche? Il y a toujours un risque d'instrumentalisation des professionnel.le.s et de leur expertise, un risque d'appropriation de cette dernière par les chercheur·e.s, ce qui nécessite une mise à plat des coûts et bénéfices pour chacun.e lors de la recherche ainsi qu'une définition en commun de la place de chacun.e. Si tel est le cas pour de nombreuses recherches en sciences humaines, dans le cas de la recherche effectuée au sein d'une haute école de travail social, la situation est particulière car, en tant que lieu de formation professionnelle, un partenariat étroit école-milieux professionnels existe; partenariat qui implique une grande proximité en ce qui concerne la participation de ces milieux à la définition et à la mise en œuvre de la formation.

Par ailleurs, la difficulté réside dans le fait que, souvent, les professionnel-le.s n'arrivent que difficilement à anticiper les étapes et les aléas d'une recherche (d'autant plus lorsqu'il s'agit d'une première participation) et ne découvrent les enjeux qu'en cours de route. Il importe d'examiner et de définir très soigneusement ces rapports, y compris lors de la valorisation des résultats, si l'on veut éviter des risques d'instrumentalisation.

La recherche-action offre à cet égard des options intéressantes, car elle oblige à expliciter et à discuter les intérêts en jeu. Et ces derniers concernent en fin de compte aussi, voire surtout, les usagères et usagers ou bénéficiaires. Car, au-delà de la relation entre chercheur.e.s et professionnel.le·s, la relation entre chercheur.e.s·et usagères ou usagers concerne notamment la façon dont sont objectivés et traités leurs vécus, leurs représentations et leurs actions, voire leur expertise, leur savoirfaire pour se débrouiller dans des situations souvent difficiles. Or, la question des enjeux de pouvoir se pose là aussi: quels sont les intérêts défendus dans la recherche? Qui contrôle l'information et son analyse? Qui rend visibles les observations et les analyses à un public plus large? A qui 
appartiennent les findings ${ }^{1}$ ou découvertes? Il en va de la question du pouvoir et de l'empowerment, à savoir: qui ressort de la recherche avec un pouvoir d'agir renforcé, une meilleure prise sur la réalité, une augmentation du pouvoir d'agir? La recherche bénéfice-t-elle aux usagères et usagers, aux professionnel-le·s, à l'organisation/institution ou aux chercheur.e.s? La définition de conditions garantissant que chacun des trois types d'actrices et acteurs soit renforcé au bout du processus est indispensable. Cela implique une transparence des relations de pouvoir, une gestion démocratique des rapports au quotidien ainsi que des espaces et moments d'inclusion, et encore d'analyse critique et d'autocritique.

\section{La restitution de la recherche}

La valorisation des recherches, le moment et le lieu où rendre publiques les problématiques étudiées, les méthodes de récolte de données, les analyses et les findings constituent des étapes importantes dans le processus de recherche, qui correspond d'ailleurs à une injonction forte du FNS. Notons que la restitution se fait en réalité tout au long du processus, en particulier auprès des partenaires de recherche, et non pas seulement à la fin, de sorte qu'elle forme un élément crucial du dispositif de recherche. Ces moments représentent des défis particuliers, car ce qui ressort d'une recherche n'est pas toujours ce que l'organisme mandant ou le public attendait.

Tout d'abord, il y a la restitution aux personnes interviewées, dont les pratiques sont l'objet de l'enquête ou qui ont été observées, ainsi qu'à celles ayant participé à la recherche; cette étape est devenue heureusement une tradition bien établie à la HETS de Genève. Elle a pour double fonction de restituer à celles et ceux qui ont «donné» et, en même temps, de vérifier les findings, de les ajuster en fonction des remarques et critiques reçues lors des présentations aux divers partenaires.

Puis, il y a une restitution plus large dans la cité, que ce soit au moyen de conférences, de publications scientifiques ou grand public, par les médias, ou encore par la création d'outils tels que des grilles de lecture pour le travail social, des outils pédagogiques, etc. Les possibilités sont diverses, mais elles ont comme dénominateur commun la nécessité de prendre en considération «d'où entend l'audience ", ce qui est véhiculé comme représentations auprès du public cible, les idées préalables concernant l'objet étudié. Les findings contredisent souvent les perceptions et attentes, voire peuvent 
même heurter les partenaires. Ils dévoilent ce qui n'est pas visible, de sorte qu'ils peuvent fonctionner comme outil de déconstruction des représentations. Mais cela ne fonctionne que dans la mesure où la façon de s'adresser à l'audience est soigneusement réfléchie et où les appréhensions ou objections sont prises au sérieux, pour que les éléments pouvant générer des tensions deviennent audibles. Dans ce sens, la valorisation représente un défi théorique et épistémologique, un travail de réflexion en soi, qui mérite d'être considéré, une sorte de "pédagogie de la transmission" avec ses concepts de dissonance, de confrontation et d'élaboration de ces derniers. C'est ainsi que la valorisation des recherches dépasse la simple communication et devient un moment de reconstruction des représentations et d'ajustement des interventions du travail social.

\section{Prolongement dans la cité}

Mais au fond, en songeant aux multiples dimensions soulevées par ces contributions, on est amené à se poser la question: à qui et à quoi sert la recherche à la HETS? Il n'y a évidemment pas de réponse facile. Dans mes propres expériences de chercheure, le prolongement dans la cité constituait un élément crucial du processus; pour nous, la finalité de la recherche résidait à long terme dans le fait que les findings élaborés au cours de la recherche permettent aux divers protagonistes de la pratique professionnelle de mieux appréhender les défis du présent et les projections dans l'avenir.

Dans les années I970 et I980, la recherche sociale était, il me semble, essentiellement vue comme outil de dénonciation, avec le souci de l'amélioration des conditions de vie et de travail de la population, en particulier de sa frange fragilisée et précarisée. Le regard paraît s'être élargi pour y ajouter d'autres dimensions, telle la compréhension des mécanismes d'exclusion, du fonctionnement des institutions, de la logique des professions et bien d'autres objets liés à l'action sociale.

1 Le terme findings, "ce qu'on a trouvé ", me paraît infiniment plus adéquat et plus ouvert que le terme de "résultat», qui a une connotation de clôture, de fermeture, qui ne correspond souvent pas à ce qui ressort d'une recherche en sciences humaines ou sociales, ou que le terme de "découvertes", qui me semble trop fortement connoté d'un aspect d'innovation. 
Est-ce que la recherche dans une haute école de travail social sert à faire avancer les connaissances des processus sociaux? Répond-elle aux besoins et aux questions du terrain? Et, si oui, aux besoins de quels actrices et acteurs de terrain: des professionnel.le.s, des directions d'institutions, des décisionnaires ou policy-makers, des usagères et usagers? Ou, plus largement, sert-elle à augmenter le pouvoir d'agir, avant tout des personnes vulnérables, mais aussi des professionnel-le.s, et ce, dans un souci de justice sociale et de démocratie?

Dans le prolongement de cette transmission, une controverse existe entre les chercheur.e.s, qui ne partagent pas toutes et tous l'idée qu'elles et ils devraient fournir, au terme d'une recherche, des recommandations ou des pistes d'intervention aux institutions professionnelles ou, plus largement, aux instances politiques. Les un.e.s préfèrent s'abstenir, s'en tenir à livrer des observations quantitatives ou qualitatives et laisser à d'autres le soin d'élaborer des politiques. Pour d'autres, il est important de contribuer à façonner les politiques publiques et institutionnelles, voire les méthodes d'intervention. Le lecteur trouvera des réponses et positions diversifiées dans ce volume.

Mais, quelle que soit la position du ou de la chercheur.e à ce propos, une éthique est à la source du travail social et lui donne constamment sens, une éthique qui constitue également une exigence pour la recherche. Celle de chercher à augmenter le pouvoir d'agir des personnes et des groupes de bénéficiaires, et cela en référence constante aux principes fondamentaux des droits humains et de la justice sociale. 


\title{
Introduction
}

\author{
Monica Battaglini, Stéphanie Fretz, \\ Eva Nada et Laurence Ossipow
}

En I969, l'Institut d'études sociales a créé son Centre de recherche sociale, le CERES. Au moment de fêter ses 50 ans d'existence, les membres de ce laboratoire proposent une réflexion sur la place et la fonction de la recherche au sein d'une Haute école de travail social en 20I8. Au fil du temps, la recherche en travail social a pris différentes formes et visé différents buts, les exigences ont évolué, cette recherche s'est affirmée. Si ce domaine d'enquête est désormais reconnu comme champ des sciences sociales, les modalités et les approches scientifiques qu'il convoque restent variées. La dizaine d'articles qui composent le présent ouvrage reflète cette diversité. Les travaux présentés poursuivent divers objectifs, qui vont de la compréhension de phénomènes et mécanismes sociétaux à l'utilisation de ces mêmes connaissances pour le fondement de l'action sociale. Aussi, ces travaux s'inscrivent dans un continuum allant de la recherche dite fondamentale à la recherche dite appliquée, en incluant des modalités telles que la recherche collaborative, l'analyse de l'activité, la recherche-action, la recherche impliquée... L'objet de ce volume n'est toutefois pas d'entrer dans un débat opposant ces deux visions de la recherche - même si celuici n'est pas clos - mais de mettre en lumière le lien que la recherche en travail social entretient avec son «sujet» d'étude, sachant que, par nécessité et par vocation, la mission de formation des hautes écoles est fortement liée aux milieux professionnels et politiques. On pourrait, comme le fait Eva Nada dans la postface de cet ouvrage ("Recherche en contexteS. Les apports du travail social aux sciences sociales ou à l'épistémologie des sciences sociales " pp. 237-252), interroger l'apport même du travail social à la recherche en sciences sociales en général. En s'attachant notamment à l'étude des marges de la société à travers une "pluralité épistémique » qui lui est propre, la recherche en travail social alimente la prise de distance et la réflexivité des chercheur·e•s, leur fournissant par ailleurs des outils 
de résistance aux pressions de leur environnement auxquelles elles et ils peuvent être confronté.e.s.

Dans ce contexte, les contributions posent la question de la spécificité de la recherche en travail social, des liens qu'elle entretient avec la cité dans laquelle elle s'insère et avec les formations dispensées dans une haute école. Les réponses à ce questionnement sont bien évidement multiples et tissent la trame de fond de cet ouvrage. Cela amène à s'interroger sur les modalités de transfert des problématiques abordées et des résultats issus des recherches. Ce transfert, ce partage, cette transmission s'avèrent tout aussi centraux dans la mission des hautes écoles. La publication, même si cette modalité est actuellement en pleine mutation (voir la contribution de Stéphanie Fretz, "A la recherche du livre perdu», pp.219-236), est sans doute un des modèles de diffusion privilégiés de la recherche en travail social et ce livre souhaite en être une illustration.

La réflexion présentée ici se compose de contributions organisées autour de deux axes. Le premier touche au lien que la recherche - dans le contexte des hautes écoles de travail social - entretient avec la cité. Tout comme les objectifs visés par les recherches, le financement de ces travaux est lui aussi varié, reposant sur des mandats privés et/ou publics ou sur des fonds de recherche académique (que d'aucun.e.s considèrent aussi comme une forme de mandat public), les deux pouvant être associés. A partir de l'axe de la cité, cet ouvrage offre une image des rapports spécifiques que les enquêtes établissent entre équipes de recherche et actrices ou acteurs de la cité - tant du côté de la genèse que de celui de la restitution -, qu'il s'agisse des institutions mandantes, des professionnel.le.s du travail social, des élu.e.s, des étudiant.e.s ou du public intéressé.

La question de la restitution de la recherche aux étudiant.e.s amène tout naturellement au second axe de cet ouvrage qui traite, lui, de la place de la recherche dans l'enseignement dans une haute école de travail social, qu'il s'agisse de la formation de base, du master ou de la formation continue. Cette réflexion permet de s'interroger notamment sur les modes de transmission des connaissances développés pour et avec les étudiant·e.s, voire sur la genèse de nouvelles problématiques naissant de ce dialogue.

Certaines contributions se rattachent clairement à l'axe de la cité et d'autres à l'axe de la formation; une circularité existant entre recherche, cité et enseignement, d'autres textes traitent des deux axes à la fois et pourraient se trouver dans l'une ou l'autre partie de l'ouvrage. Nous avons toutefois choisi de regrouper les contributions selon ces deux angles, plaçant 
en première partie les textes qui traitent principalement des relations entre la recherche dans les hautes écoles et la cité et, dans la seconde, les contributions centrées plutôt sur les liens avec l'enseignement. Une dernière section est dédiée à des questionnements plus globaux sur la recherche en travail social, mêlant des réflexions sur les modalités de diffusion et d'épistémologie.

Dans la première contribution, «Quels liens entre recherche et travail social? L'exemple des études sur l'âge et la migration" (pp. 27-43), Claudio Bolzman, spécialiste de la migration en lien avec les âges de la vie, relate comment un programme de recherche issu d'une institution spécialisée dans le financement de la recherche académique (le Fond national suisse de la recherche scientifique) lui a permis de s'intéresser, avec un groupe de chercheur.e·s, à une question encore impensée en son temps par les professionnel.le·s du travail social: celle des personnes migrantes âgées. L'exemple présenté concerne des personnes d'origine italienne ou espagnole ayant migré dans les années I950-I960 et ayant choisi de passer leur retraite dans des établissements pour personnes âgées sis dans le pays d'accueil et non pas dans le pays ou la région d'origine. Parti d'une intuition liée à leurs précédentes recherches dans le domaine de la migration, le groupe de chercheur.e.s s'est livré à une enquête sur les personnes migrantes âgées à l'aube de leur retraite, ce qui lui a permis de porter à la connaissance publique des réflexions sur la migration habituellement considérées d'abord sous l'angle du travail rémunéré. Les résultats de la recherche ont eu l'effet d'une bombe pour les personnes concernées (les personnes migrantes), mais aussi pour les institutions liées à la vieillesse, puisque celles et ceux qui les dirigent et y travaillent n'avaient pas anticipé l'accueil de ces personnes dans la perspective liée à la migration. Le groupe de chercheur.es a ensuite été chargé de deux mandats destinés à étudier comment la question du soutien aux personnes migrantes âgées était abordée en Allemagne, France et Hollande (un pays connu pour ses recherches novatrices dans le domaine). Si, dans certains cas, une première recherche sur mandat peut pousser à approfondir l'étude en utilisant des fonds liés à la recherche fondamentale, ici, c'est un programme de recherche fondamentale - qui se dit aussi tournée vers la pratique - qui a permis de déboucher sur plusieurs mandats.

Monica Battaglini et Laurent Wicht («La recherche dans les hautes écoles entre «débat académique» et «débat citoyen», pp.45-66) approfondissent 
dans leur contribution la remise en question de la distinction classique entre recherche dite fondamentale et recherche dite appliquée évoquée cidessus. Les auteur.e.s posent la distinction entre recherches davantage en fonction des objectifs poursuivis à court terme que des modalités d'enquête.

Dans ce contexte, Battaglini et Wicht montrent comment une enquête mandatée par les responsables d'un service public cantonal dévolu au logement a pu être reformulée et élargie grâce au soutien institutionnel et à la conjugaison de fonds publics (ceux des institutions mandantes) et de fonds internes HES-SO destinés à la recherche. L'enquête prise comme exemple reposait au départ sur un mandat de l'office du logement à Genève et visait à une évaluation de l'(in)satisfaction des habitant.e.s logeant dans un environnement considéré comme mixte socialement; une mixité que les responsables des politiques publiques appellent de leurs vœux, voulant à tout prix éviter les effets de ghettoïsation. En se basant sur la littérature scientifique et sur leurs propres travaux, les chercheur.e.s ont proposé une remise en question de la notion de mixité sociale, qu'elles et ils se sont attelé.e.s à redéfinir avec les commanditaires. Ensuite, l'équipe de recherche a été amenée à repenser le mandat en termes de cohabitation et non plus dans la perspective de la mixité sociale, mettant ainsi moins l'accent sur la recherche d'un équilibre entre les profils sociodémographiques des habitant.e.s que sur leurs modes de sociabilité au quotidien.

Dans la contribution suivante, la relation avec les instances mandantes est aussi centrale mais, ici, les chercheur.e.s Arnaud Frauenfelder, Géraldine Bugnon et Eva Nada ("Enquêter en "terrain difficile": production et réception d'une enquête sociologique dans un centre éducatif fermé", pp.67-88) présentent une réflexion centrée sur la production de données autant que sur la réception dans un contexte spécifique - celui d'un centre éducatif fermé pour mineur·e·s (CEF). L'équipe de chercheur.e.s qualifie ce terrain de "difficile», non pas tant parce qu'il serait particulièrement contrôlé, mais parce qu'il est exposé à différentes controverses publiques et parce qu'il a déjà été l'objet d'expertises antérieures. La difficulté des chercheur·e·s a aussi été d'avoir affaire non pas à un corps de métier et à ses potentiel.le.s client·e.s ou bénéficiaires, mais à un ensemble de professionnel-le·s (corps médical, personnel enseignant, équipe éducative, agent.e.s de détention), en concurrence ou en quête de reconnaissance, en raison de leur arrivée à des moments différents de l'évolution de l'établissement. S'expliquant sur les conditions de production de leurs données et sur les coulisses de leur recherche, les chercheure.s dévoilent, entre autres, comment elles et ils ont 
considéré l'ensemble des acteurs en évitant un biais d' «enclicage» (Olivier de Sardan, I995: 8I), c'est-à-dire d'enfermement dans un groupe plutôt que dans un autre. Par ailleurs, pour son analyse, l'équipe de recherche a utilisé le concept d' «institution totale» développé par Erving Goffman. Concept que l'équipe s'empresse de déconstruire, sans toutefois l'abandonner, car celui-ci garde notamment, de son point de vue, toute sa pertinence pour le caractère «enveloppant» qu'il thématise et qui peut s'appliquer aussi bien à l'encadrement qu'à l'accompagnement des mineur.e.s séjournant dans le CEF. Le récit de la réception des analyses de l'équipe par les professionnel.le.s révèle les difficultés à partager ce concept - même revisité - avec eux, comme si la notion d'«institution totale» était passée dans le langage commun (en tout cas dans celui des professionnel.le.s du carcéral) et ne pouvait qu'être rejetée avec l'idée d'enfermement global qu'elle suppose.

En lien avec le domaine des migrations, Théogène-Octave Gakuba («Posture du ou de la chercheur.e dans la relation d'entretien avec des jeunes ayant des parcours migratoires difficiles", pp.89-I08) se penche sur la question de la souffrance des jeunes associée à leur trajectoire migratoire; il rencontre certaines personnes lors d'une enquête portant sur l'exil de réfugié.e.s rwandais tandis que d'autres jeunes réfugié.e.s sont issus de l'immigration d'autres pays d'Afrique sub-saharienne. Même si le chercheur s'interroge sur le processus d'intégration des personnes avec lesquelles il s'est entretenu, le cœur de son article réside dans le mode d'entretien proposé aux interviewé.e.s. En répondant aux questions ou en narrant tout ou partie de leur vie, les jeunes revivent des souffrances liées à leur trajectoire. Ces personnes ne se sentent en effet pas reconnues dans leur souffrance, qu'elle soit due à l'exil, à l'arrivée dans le pays dit d'accueil ou, plus tard, une fois naturalisées. Quel que soit leur mode d'intégration, ces personnes souffrent de racialisation ou de racisme, notamment du fait de leur couleur de peau. Dès lors, par l'organisation de conférences de restitution et de diffusion des résultats, le chercheur a souhaité, entre autres, rendre publiques les souffrances liées à l'émigration et à l'intégration dans le pays de résidence.

Dans la deuxième partie de cet ouvrage, sont regroupées les contributions qui traitent principalement des liens entre les recherches et la formation, bien que comme nous le soulignions au début de notre présentation, les textes qui concernent l'enseignement en haute école de travail social soient 
bien sûr aussi ancrés dans la cité. Former les futur·e·s professionnel.le·s de l'action sociale est une tâche que les hautes écoles assument, bien évidemment, pour et avec le reste de la société. Toutefois, les préoccupations de certaines contributions concernent plus directement les visées pédagogiques, leur déroulement et leurs effets espérés ou effectifs. C'est le cas des cinq textes suivants.

Dans la première contribution de cette seconde partie, Laurence Ossipow («La recherche anthropologique dans l'enseignement en travail social», pp. I I I-I 29) présente une réflexion sur son enseignement autour des notions anthropologiques et sociologiques d'échanges et de réciprocité, d'habitus, de culture et de rites. Elle montre, à travers différents exemples de recherches, leur portée heuristique pour l'analyse du travail social, favorisant par-là l'engagement et la participation des étudiante-s dans le cours. En effet, les notions développées par des anthropologues et des sociologues de renom, adoptées pour l'analyse du travail social, suscitent la réflexion des étudiant·e·s sur leurs propres pratiques, développant leur réflexivité au point d'emprunter, par exemple, la notion de don - étudiée dans le module - pour en faire le thème de leur soirée de fin d'année. Mettant en évidence des formes d'appropriation inattendues par les étudiant.e.s ainsi que, parfois, leurs critiques sur la démonstration, Laurence Ossipow souligne tout l'intérêt pour la formation en travail social de donner la possibilité aux étudiant.e.s d'étudier et décortiquer des notions théoriques classiques, puis de les mettre en débat avec les enseignant·e·s et les professionnel.le.s du social.

Dans une même perspective de recherche et d'enseignement, Francis Loser («La posture professionnelle et la réflexivité en travail social envisagées sous l'angle ethnographique et esthétique», pp. I3I-I50) part de sa propre posture d'observateur sur le terrain d'une enquête liée aux domaines de l'art et du handicap et s'interroge sur ses perceptions corporelles et cognitives ainsi que sur ses émotions, avant d'expliquer comment il les relate dans son journal de terrain pour les utiliser ensuite dans un cadre analytique. Mettant l'accent sur les ressentis corporels qui peuvent faciliter la perception, la compréhension et la représentation, il montre comment les mêmes processus peuvent être vécus par les étudiant.e.s qui suivent son cours, les contraignant, par exemple, à faire l'expérience personnelle d'un déplacement en fauteuil roulant dans la cité en rencontrant des obstacles et des appuis, mais aussi parfois des attitudes stigmatisantes. Cette expérience est alors à relater puis à expliquer dans le cadre 
d'un enseignement non point tourné vers la recherche mais pensant la posture professionnelle.

Dans une autre perspective, Sylvie Mezzena et Kim Stroumza («Faire se rencontrer recherche, intervention et formation: effets en cascade d'un projet de recherche", pp. I5I-I72) montrent le lien étroit entre le processus de recherche et la formation. Cette dernière étant, pour les actrices et acteurs, l'essence même de toute la démarche. Les auteures décrivent comment l'équipe de recherche, dans le cadre d'un mandat de recherche, associe dès le début de l'enquête les actrices et les acteurs du champ d'activité considéré (le travail des équipes éducatives de la petite enfance, des parents et des enfants autour de la question de l'intégration). Comme le soulignent les chercheures, leur façon de mener la recherche table sur l'intérêt des professionnel.le·s pour la thématique de l'enquête que lesdit.e.s professionnel.le.s choisissent d'aborder comme elles et ils le souhaitent, tant dans leurs propos que dans les activités qu'ils ou elles laissent observer aux chercheur.e.s. On notera que ce mode de faire est assez analogue, épistémologiquement parlant, à celui d'une partie des anthropologues et sociologues. Il s'agit, en effet, de partir du point de vue émique (ou indigène) des personnes sujettes et participantes de l'enquête. Ce point de vue présente de l' «incertitude» (on ne sait jamais ce qui adviendra dans les situations observées, ni comment la recherche va évoluer), de la "confiance» (tout le long de l'enquête, il faut faire confiance aux chercheur.e.s et à ses propres collègues, comme aux autres participant.e.s) et de l'«engagement» puisque les protagonistes ne se contentent pas de répondre à un certain nombre de questions émanant d'un guide d'entretien, ni d'être observé.e.s passivement. En fin de compte, même si, souvent (et en tout cas dans le mandat décrit), des textes sont produits, des formations sont organisées et des films ou des outils multimédias sont conçus et diffusés, les professsionnel.le·s concerné·e.s ne se voient pas obligé.e·s d'appliquer d'hypothétiques recommandations qui auraient été faites par les chercheur·e.s à l'issue de l'enquête. Comme l'affirment les chercheures: "Nos interventions visent à construire une connaissance des activités, et c'est aux professionnel.le.s de se saisir (ou non) du dispositif de recherche pour faire (ou non) évoluer leurs pratiques» (Mezzena et Stroumza: I59).

Dans leur article sur le rapport à l'argent des professionnel.le.s du travail social (ou plus précisément, des assistantes et assistants sociaux - AS) encadrant des situations de surendettement, Sophie Rodari et Laurence Bachmann («L'imbrication heuristique entre professionnel.le.s du travail 
social, recherche et enseignement», pp. I73-I9I), expliquent d'une autre manière comment formation et recherche s'entremêlent. C'est d'abord dans le cadre de formations estudiantines et professionnelles que la réflexion s'est ancrée, pour être ensuite développée sous forme de recherche. L'étude montre que les pratiques des AS sont variées et ne représentent pas un simple instrument de contrôle au service des institutions qui les emploient, mais bien plutôt une source d'accompagnement, de soutien et d'encouragement. Toutefois, seul.e.s les professionnel.le.s les plus militant.e.s se préoccupent plus largement des rapports sociaux et de genre qui structurent les inégalités auxquelles les personnes endettées doivent faire face. Au vu du peu de temps dont disposent généralement ces professionnel.le.s, se pose donc la question de savoir comment elles et ils pourraient s'approprier plus largement les analyses des chercheur.e.s sur ces questions. Ainsi, la recherche renvoie directement au champ du travail social et aux politiques qui l'encadrent. Par ailleurs, les chercheures mettent en évidence comment la construction de cette recherche s'appuie sur la collaboration entre deux profils professionnels de recherche différents. Le profil de la première est celui d'une ancienne praticienne ayant un fort ancrage auprès des terrains professionnels, la seconde présente un profil marqué par l'«excellence académique» (expérience de recherche financée par des fonds académiques, séjours dans des universités prestigieuses, publications scientifiques, etc. ). Ce croisement de regards, cette mixité de profils au sein de l'équipe de recherche constitue, selon les chercheures, une manière intéressante de répondre au défi de l'articulation entre demande de la cité, enseignement et recherche qui se pose dans les hautes écoles spécialisées.

Manon Masse («Prévenir la maltraitance envers les personnes vulnérables: de la recherche à la formation ", pp. I93-2I5) présente deux recherches: une première, menée auprès de trois groupes d'actrices et acteurs (les personnes en situation de handicap, les proches et les professionnel.le.s) pour circonscrire la notion de maltraitance en milieu institutionnel et une seconde, qui consiste en une évaluation de la formation menée à la HETS sur les questions liées à la maltraitance. Dans celle-ci, la chercheure montre clairement comment un programme de formation impliquant activement les participant.e.s - qui, à leur tour, devaient imaginer un programme de formation - peut avoir un impact fort sur les représentations et les interventions des étudiant.e.s. En effet, et par comparaison avec un groupe contrôle d'étudiant·e·s qui n'avaient pas reçu cette 
formation, les participant·e.s élargissent et affinent considérablement leurs représentations de la maltraitance et s'impliquent même dans des débats ou des actions directes autour de cette question.

La recherche et l'enseignement dans les hautes écoles sont, comme la science en général, liées à l'histoire, au contexte, aux modes et controverses (Karsz, 20I7). Ainsi, les textes présentés dans ce volume n'ont pas vocation à arrêter des positionnements de portée générale. Ils visent plutôt à interroger, voire à déconstruire - chacun à partir d'exemple précis - la notion de frontière, même poreuse, entre les types de recherches. Ils montrent ainsi que la rupture épistémologique classique entre l'analyse des scientifiques et les perspectives des professionnel.le.s du travail social n'apparaît pas comme pertinente. La recherche endosse, dès lors, une forme collaborative qui reconnaît aux pratiques et aux savoirs des professionnel.le.s toute leur importance. Outil à visée démocratique et émancipatoire, la recherche en travail social s'insère dans un cercle vertueux auquel cité et formation appartiennent; sorte de valse à trois temps qui implique un repositionnement constant face à une société en rapide et perpétuel mouvement. 

La recherche dans la cité Première partie 



\section{Quels liens entre recherche et travail social?}

L'exemple des études sur l'âge et la migration

\section{Claudio Bolzman}

La question des liens possibles entre recherche et travail social fait débat, notamment dans le monde francophone. Ainsi, lors de la Conférence de consensus $^{1}$ qui a eu lieu en France en 2012, il était question de savoir si l'on devait développer la recherche «dans, sur ou en travail social» (Jaeger \& Mispelblom Beyer, 20I4), comme s'il s'agissait de positions exclusives et inconciliables. Dans le même sens, il m'est arrivé aussi de rencontrer des collègues pour qui la seule recherche légitime concernant le travail social est celle qui étudie l'action des professionnel.le.s et ses effets. En revanche, d'autres collègues estimaient que le rôle des chercheur.e.s était d'analyser à partir d'une position d'externalité et surplombante ce qui se passe dans le monde social. En Suisse, on discute par ailleurs, bien que de manière moins figée et plus pragmatique, ${ }^{2}$ sur la nécessité d'élaborer une recherche dite appliquée qui répondrait mieux aux besoins des terrains que la recherche dite fondamentale.

Mon expérience pratique de chercheur et enseignant au sein du Centre de recherche sociale de la Haute école de travail social de Genève m'amène à avoir une position plus nuancée sur ces débats. En effet, la thèse que

1 La fonction explicite de cette Conférence de consensus était de rapprocher les perspectives à propos du lien entre recherche et travail social, car la question est très controversée en France.

2 Sur cette question, le Fonds national suisse de la recherche scientifique (FNS) a choisi d'intégrer la recherche qu'il a définie comme «recherche orientée vers l'application" dans la Division des sciences humaines et sociales, qui s'occupe en principe de la recherche dite fondamentale. Par ailleurs, les critères pour évaluer les deux types de projets de recherche sont, à quelques nuances près, identiques, mettant ainsi en évidence que le FNS ne perçoit pas une différence de nature entre ces deux types de recherche. 
je défends dans cet article est que, selon les circonstances, les questions posées, l'origine de la demande, etc., les mêmes chercheur.e.s peuvent être amenés à développer des modes de recherche différents et que tous peuvent être pertinents pour le travail social, à condition que des articulations soient construites entre professionnel-le.s de la recherche et professionnel.le.s de l'intervention. Ainsi, parfois, la recherche dite fondamentale peut contribuer à la production des savoirs nécessaires au travail social, car elle permet de découvrir des problématiques ignorées par l'intervention ou de penser autrement certains problèmes. Parfois, en revanche, c'est la collaboration étroite entre chercheur.e.s et instances sociales, ou entre chercheur.e.s et professionnel.le.s, voire entre chercheur.e.s et étudiant.e.s qui permet de produire des connaissances nouvelles et d'élaborer des méthodes d'intervention plus adaptées (Bolzman, 2008; 2014). A partir de recherches auxquelles j'ai participé autour des questions de l'âge et de la migration, on trouvera, dans ce qui suit, plusieurs illustrations de cette thèse.

\section{Le contexte de départ: une rencontre improbable entre vieillissement et migration}

Aussi bien les questions relatives au vieillissement de la population que celles liées aux migrations intéressent au premier chef le travail social et, plus largement, les politiques sociales. En effet, la problématique d'une société composée d'un nombre croissant de personnes âgées et le financement des pensions de vieillesse et de retraite occupent souvent l'actualité de débats. Il en va de même pour les problématiques liées à l'intégration des personnes migrantes. Cependant, aussi bien au niveau de la réflexion sur les politiques à mettre en place que sur les terrains d'intervention, mais également sur le plan de la recherche, ces deux problématiques ont rarement été prises en considération ensemble jusqu'à une période relativement récente. Cela était clairement le cas lorsque, avec des collègues, j'ai commencé des recherches sur cette thématique en I992. Ainsi, au début des années I990, un démographe, Olivier Blanc, s’intéressait aux défis auxquels la Suisse devrait faire face dans le futur. Parmi ceux-ci, il mentionnait «Le vieillissement de la population résidente et la permanence d'une part d'étrangers représentant environ un habitant sur six (qui) posent des questions de société délicates mais ne pouvant 
pas être éludées ou contournées» (Blanc \& Gilliand, I99I : I5). Blanc se contentait d'évoquer en parallèle le vieillissement de de la population et la présence permanente des personnes étrangères, sans aborder explicitement la question des personnes âgées immigrées. Un peu plus tard, dans le Rapport fédéral suisse sur le vieillissement publié en I995 - un rapport de plus de trois cents pages - nombre de questions de société liées à la vieillesse étaient abordées, mais sans aucune référence aux personnes âgées immigrées.

Lorsqu'on établissait une relation entre les deux problématiques, la migration était perçue comme une manière de contrecarrer potentiellement le vieillissement de la population et ses conséquences sur les politiques sociales. En effet, on percevait la population immigrée - et c'est d'ailleurs encore largement le cas - comme une force de travail qui pouvait contribuer à rééquilibrer la structure par âge de la population et à financer l'assurance vieillesse et survivants (AVS). Ainsi, en I993, un député demanda avec inquiétude au Conseil fédéral si une proportion de $40 \%$ des étrangers serait nécessaire en 2040 pour financer l'AVS. Il estimait cependant que «ces personnes finissent par vieillir aussi» (Stalder, I993: 40). On notera que le conseiller national projetait le phénomène dans un temps futur. En fait, les personnes immigrées étaient perçues principalement en fonction de leur rôle économique de travailleuses, consommatrices, contribuables et cotisantes aux assurances sociales (Bolzman, I999). Leur vieillissement était invisible.

Au niveau des instances sociales et des professionnel-le.s du travail social, le lien entre ces deux problématiques (vieillissement et migration) n'était également guère perçu. Ainsi, les structures destinées aux personnes âgées n'abordaient pas la thématique des personnes âgées migrantes, ou alors comme un phénomène très marginal. De leur côté, les structures destinées aux personnes migrantes estimaient que le centre de leur intervention devait se concentrer sur les personnes actives et leurs familles. Lorsque nous avons commencé à nous intéresser aux personnes âgées migrantes, nous ne pouvions donc pas faire appel aux pratiques de travail social existantes ni aux expériences des professionnel.le·s du travail social dans le domaine car ce n'était pas une problématique identifiée comme telle. 


\section{La recherche "fondamentale" comme révélatrice d'une problématique méconnue du travail social: les personnes âgées immigrées}

Ainsi, lorsque nous avons débuté dans ce domaine de recherche, nous ne pouvions que nous appuyer sur l'initiative de notre groupe de chercheur.e.s de l'Ecole de travail social (alors Institut d'études sociales). Au début des années I990, le FNS lança un appel à projets de recherche dans le cadre d'un Programme national suisse de recherche (PNR) intitulé «Vieillesse». Avec mes collègues Rosita Fibbi et Marie Vial, nous décidâmes de postuler à ce programme à partir de l'exploration de la question du retour pour les personnes immigrées espagnoles et italiennes proches de la retraite. ${ }^{3}$ L'idée fut retenue par les experts de ce PNR car, jusqu'à ce moment-là, personne ne s'était intéressé au vieillissement de ces populations arrivées pour travailler en Suisse entre les années 1950 et I960. Il s'agissait ainsi d'une démarche scientifique "classique», issue du monde académique. La problématique et les méthodes étaient du ressort exclusif des chercheur.e.s, qui ont pensé le projet à partir de leurs intuitions, de leurs connaissances théoriques, d'un travail d'examen systématique de la littérature scientifique suisse et internationale sur le thème et des contacts exploratoires avec la population directement concernée. La principale méthode de récolte des données consista en une enquête par questionnaire (en partie autoadministré, en partie face-à-face) à laquelle répondirent quatre cent quarante-deux personnes espagnoles et italiennes âgées de 55 à 64 ans et résidant dans les cantons de Genève et Bâle-Ville. Il y eut aussi une vingtaine d'entretiens semi-directifs auprès d'une partie des enquêté.e.s. Les résultats ont rendu attentifs les professionnel.le.s et les institutions sociales à l'existence d'une population qui, contrairement aux conceptions habituelles, ne rentrait pas dans son pays d'origine au moment de la retraite et qui se trouvait en situation précaire, du point de vue économique et de son état de santé, par rapport aux personnes âgées «nationales» (Bolzman, Fibbi \& Vial, I996; I999). Une population qui avait donc besoin davantage que d'autres du soutien des services sociaux et qui pourtant les sousutilisait (Bolzman et al., 2004).

Cet exemple, présenté de manière très sommaire et schématique, met en évidence qu'il est parfois nécessaire de poser des questions qui a priori peuvent paraître éloignées des préoccupations immédiates du travail social et qui demeurent plus largement sous-estimées dans l'imaginaire social. 
Notre recherche a pu montrer l'influence de la politique d'immigration (Sayad, I99I) sur cet impensé social à propos des personnes immigrées âgées. En effet, les populations venues dans les années I950 et I960 furent recrutées dans le cadre d'une politique dont l'objectif premier était de disposer d'une main-d'œuvre temporaire, bon marché et utile à court ou à moyen terme pour l'économie. Nul ne songeait alors à une immigration permanente. Dans ce contexte, le regroupement familial fut accepté avec beaucoup de retard et, en Suisse comme dans d'autres Etats européens, il n'y eut guère de politiques d'intégration mises en place au bénéfice de la "première génération " d'immigré.e.s; cette tâche fut abandonnée au hasard des trajectoires personnelles, sans que des mesures concrètes ne viennent encourager la participation des migrant.e.s à la vie sociale et culturelle du pays de résidence. Cette situation ne préoccupait pas outre mesure les autorités, puisque celles-ci n'avaient pas envisagé sérieusement que ces «travailleuses et travailleurs » demeureraient à long terme dans la société de résidence. D’ailleurs, le terme allemand pour les désigner était celui de Gastarbeiter, «travailleur invité», donc forcément de passage. La recherche a donc permis de faire émerger une problématique à laquelle le travail social n'avait accordé qu'une attention discrète jusque-là.

\section{De la recherche fondamentale à la recherche mandatée}

La mise en lumière par notre recherche de cette invisibilisation d'une problématique sociale a fait l'effet d'une «bombe» dans les milieux concernés par le travail social auprès des personnes âgées, mais aussi auprès des personnes immigrées, qui s'étaient concentrées plutôt sur les personnes en âge d'exercer une activité ou les mineur·e•s. La diffusion de ces résultats inattendus provoqua une remise en question des instances intervenant auprès des personnes âgées ou des personnes migrantes. Nous avons ainsi été mandatés dans un deuxième temps par deux organismes importants en Suisse - Pro Senectute Suisse ${ }^{4}$ et la Commission fédérale

3 Projet de recherche $\mathrm{n}^{\circ}$ 4032-35679 du FNS, "Modes de vie et projets d'avenir des immigrés espagnols et italiens proches de la retraite», dans le cadre du PNR 32 de Claudio Bolzman et Rosita Fibbi, obtenu en I992.

4 Fondation sans but lucratif mandatée par la Confédération suisse pour développer des prestations d'aide sociale auprès de la population âgée. 
des étrangers ${ }^{5}$ - pour donner suite à notre première étude et entamer une recherche, cette fois-ci appliquée, sur les pratiques de soutien existantes dans certains pays européens à l'égard des personnes immigrées âgées, dans le but de trouver des sources d'inspiration pour la Suisse. ${ }^{6}$ Nous avons pris le cas de deux pays voisins - l'Allemagne et la France - et d'un pays réputé pour sa réflexion pionnière dans le domaine, la Hollande. A partir d'une recherche documentaire dans ces trois pays et d'entretiens semi-directifs avec des professionnel-le.s des organismes du travail social dans ces pays, nous avons rédigé un rapport intitulé «Expériences européennes par et pour les migrants âgés» (Fibbi, Bolzman \& Vial, I999). Ce rapport, traduit en allemand, ${ }^{7}$ a été discuté dans le cadre d'une première conférence nationale sur les personnes immigrées âgées à laquelle ont participé des représentant.e.s de nombreux organismes de travail social, en particulier auprès des personnes âgées et auprès des personnes immigrées, ainsi que des représentant.e.s des associations d'immigré.e.s. Cette conférence a donné lieu un peu plus tard à la création d'un Forum national "Age et Migration", réunissant des représentant.e.s des principaux organismes concernés par l'action sociale et de santé dans le domaine de la vieillesse et de l'immigration en Suisse. J'ai été intégré en tant que chercheur à ce forum, qui a donné une impulsion à divers projets de soutien aux personnes immigrées âgées. On peut citer, à titre d'illustration, la mise en œuvre des "pôles méditerranéens" dans plusieurs établissements médico-sociaux (EMS) de villes en Suisse alémanique, qui s'inspiraient des exemples mis à jour par notre rapport de recherche. Il s'agissait notamment de permettre aux personnes âgées italiennes et, dans une moindre mesure, espagnoles, qui le souhaitaient de se retrouver dans un espace où elles pouvaient pratiquer leur langue et poursuivre certaines habitudes alimentaires ou familiales susceptibles de favoriser une meilleure qualité de vie durant cette période délicate de leur existence. On reconnaissait ainsi qu'égalité de traitement ne voulait pas dire uniformité de traitement et que des besoins de base pouvaient être satisfaits de manière spécifique. Plutôt que de demander aux personnes âgées immigrées de s'adapter aux institutions, on tenta des expériences d'ouverture interculturelle des institutions, comme le recommandait notre rapport de recherche. Il est à noter, en effet, que toutes ces expériences de "pôles méditerranéens " ont été conçues par des EMS destinés à toutes les personnes âgées. Les personnes âgées italiennes et espagnoles disposent d'un espace à elles dans des bâtiments où se retrouvent aussi des 
personnes âgées suisses et d'autres nationalités. Les prestations sont donc les mêmes pour toutes et tous, et des contacts peuvent avoir lieu entre des personnes de différentes nationalités qui le souhaitent. Enfin, il n'y a pas d'obligation pour les personnes italiennes et espagnoles de choisir ces "pôles méditerranéens". ${ }^{8}$

Durant la même période, nous avons aussi été sollicités par un autre organisme, l'Office de coordination des Ecoles-clubs Migros, ${ }^{9}$ afin de mener une recherche exploratoire sur la pertinence de mettre en place des activités de loisirs et de formation adressées spécifiquement aux personnes âgées immigrées. Notre mandataire avait l'impression que les personnes âgées espagnoles et italiennes étaient peu présentes parmi le public des Ecoles-clubs Migros. Sa coordinatrice, d'origine italienne, était particulièrement sensible à cette question et s'était intéressée à la thématique suite à la présentation publique de notre recherche FNS. Nous avons négocié le mandat avec elle et avons obtenu de ne pas cibler uniquement les activités de cette Ecole-club, mais de prendre en considération également la participation de ces personnes âgées à d'autres instances, davantage en lien avec l'animation socioculturelle, telles que les maisons de quartier ou les clubs des aînés. Etant donné que le mandataire

5 Devenue aujourd'hui la Commission fédérale des migrations. Il s'agit d'un organisme créé et financé par la Confédération pour promouvoir l'intégration des personnes étrangères en Suisse.

6 Le mandat de Pro Senectute et de la Commission fédérale des étrangers a été financé par le Pour-cent culturel Migros et a été confié à l'Institut d'études sociales. La recherche a commencé en 1999.

7 Ce qui est important puisque deux tiers de la population suisse sont germanophones.

8 Certaines critiques sur les risques de créer des "ghettos" pour personnes immigrées âgées sont apparues. Cependant, ce n'est pas lorsque les personnes âgées se trouvent fragilisées ou en état de dépendance que la cohabitation avec des personnes d'autres origines et qui parlent une autre langue est la plus facile. La situation de ces personnes à ce stade avancé de leur parcours de vie reflète plutôt l'absence de politique d'intégration à leur égard lorsqu'elles ont travaillé comme jeunes adultes en Suisse.

9 Etude réalisée à la demande de l'Office de coordination des Ecoles-clubs Migros, Zurich, à l'Institut d'études sociales et financé par le Pour-cent culturel Migros, organisme à but non lucratif qui finance des projets culturels et sociaux, principalement en Suisse. L'étude a commencé en 2000. 
demandait un rapport dans des délais brefs, la principale méthode utilisée fut celle des focus groups: dans les deux cantons de l'étude - Genève et Bâle-Ville -, neuf groupes ont été mis en place, à savoir un groupe d'hommes, un groupe de femmes et un groupe mixte pour chacune des nationalités (espagnole, italienne, suisse). La dimension comparative de l'étude, mettant aussi en évidence les pratiques des Suissesses et Suisses âgés, a permis de dégager certaines spécificités des manières de structurer la vie quotidienne de la part des personnes âgées espagnoles et italiennes, portant non seulement l'empreinte de leur origine nationale, mais également de leur origine sociale. Nous avons observé, par exemple, l'importance d'activités propres à la culture populaire, qui permettent de joindre «l'utile à l'agréable » et où la dimension familiale est souvent présente (Hoggart, I970).

Du point de vue du travail social, les résultats confirmaient la faible présence des personnes âgées espagnoles et italiennes dans les clubs des aînés et les maisons de quartier. Les participant.e.s à l'étude estimaient que ces lieux "n'étaient pas pour des personnes comme eux». Ils évoquaient leur souhait d'espaces de rencontre informels où il leur serait possible de se rendre sans crainte de se sentir mal à l'aise. Au fond, tout comme dans le cas des EMS, ces personnes exprimaient la nécessité d'un espace où elles n'auraient pas à justifier de leur intégration et qui serait adapté aux besoins des personnes âgées immigrées issues de milieux populaires. Le message renvoyé aux instances existantes était donc la nécessité de repenser le travail d'animation socioculturelle avec un public vieillissant devenu plus divers (Bolzman, Fibbi \& Vial, 200I).

Les deux exemples sont donc issus des recherches mandatées, ciblées spécifiquement sur des questions d'intérêt pour les mandant.e.s. Mais ces deux études n'auraient probablement pas eu lieu sans l'existence d'une recherche "fondamentale» qui les avait précédées et avait inscrit la problématique dans l'agenda des préoccupations sociales. Par ailleurs, les chercheur.e.s ont certes rempli leur mandat, mais ils ont en même temps inscrit leurs résultats dans le cadre du débat scientifique avec leurs pairs, au sein des colloques ou des séminaires scientifiques, et ont contribué à des publications inscrivant les résultats empiriques dans un cadre théorique plus large. 


\section{Recherches en collaboration avec des travailleuses et travailleurs sociaux}

Nos divers travaux et la diffusion que nous en avons faite, notamment à travers nos interventions dans les formations de Bachelor, Master ou postgrade, ont attiré l'attention de travailleuses et travailleurs sociaux qui se sont sentis interpellés par des situations rencontrées dans le cadre de leur activité professionnelle. Les échanges avec une partie de ces professionnel.le.s se sont limités à des discussions informelles. D'autres ont choisi d'aller un peu plus loin dans leur réflexion et de profiter du cadre d'une formation pour faire leur mémoire de fin d'études sur des questions relatives aux personnes âgées immigrées (Jäggi, 2004; Lopes, 2006). Certain.e.s ont aussi développé des initiatives spécifiques destinées à améliorer la situation de ce public dans leurs institutions respectives. Enfin, quelques travailleuses et travailleurs sociaux ont établi des discussions plus suivies avec nous, qui nous ont progressivement amenés à élaborer des collaborations dans le cadre de recherches systématiques.

Diverses recherches que nous avons menées ont en effet été suscitées directement par les questions de travailleuses et travailleurs sociaux en lien avec la grande complexité qui caractérise certaines situations rencontrées par les populations âgées de nationalité étrangère; en lien parfois aussi avec des dilemmes éthiques concernant les effets de leurs interventions sur la vie des personnes concernées. Nous avons ainsi été sollicités pour clarifier certaines interrogations par rapport à ce que l'on pourrait définir comme des épreuves institutionnelles spécifiques à certaines catégories de populations immigrées. On peut définir les épreuves institutionnelles comme des exigences que les institutions imposent à ces populations, souvent en relation avec leur droit au séjour ou leurs droits sociaux, et qui viennent s'ajouter aux épreuves que tout individu peut rencontrer dans son parcours de vie (Bolzman, 20I4). Un nombre important de mes recherches se concentre sur cette problématique. Dans le cadre de cet article, j'évoquerai deux exemples liés à la thématique âge et migration: l'un concerne le regroupement familial des ascendant.e.s, l'autre la précarité des fonctionnaires internationaux à la retraite.

Pour ce qui est du premier exemple, une assistante sociale travaillant dans un service social pour personnes âgées a constaté que son institution recevait un nombre croissant de familles ayant fait venir une 
mère âgée ${ }^{10}$ en Suisse et qui n'arrivaient plus à faire face toutes seules aux problèmes engendrés par cette nouvelle situation. D'une part, les professionnel.le.s n'arrivaient pas à bien cerner ces situations; d'autre part, ils ne savaient pas très bien quelle était leur marge de manœuvre pour venir en aide à ces familles. Une assistante sociale nous sollicita pour clarifier cette situation. Elle ne se contenta pas simplement du rôle de «lanceur d'alerte", mais s'impliqua pleinement dans la démarche de recherche. Suite à l'élaboration d'un projet de recherche, nous avons obtenu le financement du Fonds DORE du FNS pour mener à bien une recherche «classique ". ${ }^{11}$ Afin de garantir la participation de l'assistante sociale dans le processus, nous avons soutenu ses négociations avec son employeur pour qu'elle puisse consacrer du temps à la recherche dans le cadre de ses heures de travail. Le fait de l'avoir impliquée, ainsi que sa direction, dès le début du projet de recherche a facilité la négociation. Elle a obtenu d'être déchargée de ses tâches une demi-journée par semaine. Par rapport aux exigences de la recherche, cela peut apparaître comme un temps relativement limité, mais pour elle comme pour les quatre autres personnes qui formaient l'équipe de recherche, ce temps se révéla précieux. En effet, elle participa à toutes les séances de l'équipe de recherche et sa connaissance concrète des situations et de ce qui se passait dans les institutions pour personnes âgées fut très importante pour l'équipe et pour la bonne marche de la recherche. En effet, lorsque des membres de l'équipe allaient "à la pêche" aux situations de regroupement familial d'un·e ascendant.e, par exemple, ses liens avec d'autres assistantes et assistants sociaux et sa connaissance de la problématique rendaient attentifs ses collègues intervenant.e.s au fait qu'il y avait plus de situations de ce type qu'il n'y paraissait dans leurs dossiers. Son action a donc permis de rendre visibles des situations qui seraient restées dans l'ombre sans son intervention. $\mathrm{Au}$ total, nous avons interviewé quarante-huit professionnel-le.s et vingttrois familles concernées par le regroupement familial d'un·e ascendant.e. Cette assistante sociale participa également à des séances d'analyse de cas où les savoirs des uns (professionnel.le.s de la recherche) et des autres (professionnel.le·s de l'intervention) ont été précieux pour l'exploration de la problématique. Enfin, elle cosigna notre principal article de synthèse de la recherche, qui a été publié en français et en anglais (Bolzman et al., 2008a; 2008b).

Les résultats ont mis en évidence que la figure de l'ascendant.e âgé.e venu.e rejoindre ses enfants est inexistante dans la Loi sur les étrangers et 
dans les statistiques sur le regroupement familial. Ce qui reflète un décalage entre les réalités transnationales que ces familles ont vécues pendant des années (puisque leurs membres ont vécu la solidarité - envois réguliers de fonds à la personne âgée - et l'intimité à distance - contacts téléphoniques ou via internet) et les réponses institutionnelles, basées sur un concept de famille bien plus restreint et sur des politiques sociales liées étroitement à la notion de solidarité nationale (Righard, 2008). Cette épreuve qui conduit à s'occuper d'aîné.e.s en situation de fragilité ou de dépendance concerne de nombreuses familles. Elle revêt un caractère particulier et plus complexe pour les familles avec un membre étranger (Bolzman et al., 2008a; 2008b).

Outre le fait d'avoir diffusé les résultats de la recherche sous une forme classique (articles scientifiques, tels que Vuille et al., 2013; Bolzman, 20I5), la présence d'une assistante sociale dans l'équipe a permis d'adapter et d'ajuster progressivement les interventions des professionnel.le.s de son institution à partir des nouvelles informations issues de l'enquête de terrain. Elle a permis aussi de rendre plus visible la problématique auprès de ses collègues directs et des membres de son réseau professionnel. Avec les nouvelles connaissances acquises grâce à l'étude, l'assistante sociale est devenue une personne de référence pour les professionnel.le.s concerné.e.s par la problématique.

Un deuxième exemple, qui sera un peu moins développé ici car la recherche n'est pas tout à fait terminée, concerne la problématique d'une "précarité inattendue " chez les anciennes et anciens fonctionnaires internationaux. J'ai été alerté sur cette question, en effet, par une ancienne étudiante devenue assistante sociale pour l'Association d'anciens fonctionnaires internationaux, qui suivait, dans le cadre de cette fonction, un certain nombre de «cas difficiles" caractérisés par une précarité financière, de la solitude, parfois accompagnées par des problèmes de santé et des difficultés de communication au niveau local dues à une connaissance lacunaire du français. A la suite de nombreuses réunions informelles, nous avons décidé d'élaborer un projet de recherche pour approfondir cette

Le plus souvent, il s'agissait de femmes, du fait de leur espérance de vie plus longue, mais il y avait aussi quelques cas concernant des hommes ou des couples âgés.

11 Projet nI3DPD3-I I 2658 FNS, «Regroupement familial des ascendants et travail social», de Claudio Bolzman et Elisabeth Hirsch Durrett, obtenu en 2006. Partenaires de terrain: Pro Senectute Genève et Pro Senectute Vaud. 
problématique inattendue. En effet, l'image qui prédomine habituellement est celle d'une population assez aisée et bénéficiant de conditions de vie généralement agréables. C'est le réseau CEDIC de la HES-SO qui a financé la mise en œuvre de ce projet. ${ }^{12}$ Nous avons élaboré un questionnaire auto-administré destiné à tous les membres de l'association et avons mené aussi des entretiens semi-directifs avec d'anciennes et anciens fonctionnaires internationaux résidant dans la région du "Grand Genève ». ${ }^{13}$

L'élaboration du questionnaire s'est avérée extrêmement complexe. Il fallait en effet produire un instrument en deux langues, anglais et français, avec une traduction "parfaite» pour les rendre comparables et ne pas biaiser les réponses. De plus, l'association voulait avoir un droit de regard sur le contenu du questionnaire et, par le truchement d'un des membres de la direction, elle est intervenue à plusieurs reprises pour demander des modifications de celui-ci. Cela a retardé considérablement le travail de l'équipe de recherche et produit un certain découragement parmi ses membres. Sans l'intervention diplomatique et constructive de l'assistante sociale, par ailleurs bilingue et très impliquée dans la construction du questionnaire, le processus aurait demandé encore plus de temps. En outre, sa connaissance du milieu s'est avérée précieuse pour pouvoir intervenir dans une séance d'information destinée aux membres de l'association et se livrer à une première distribution du questionnaire. De plus, elle a réussi à convaincre la direction de l'association de l'envoyer par courrier électronique à l'ensemble de ses membres. Enfin, sa connaissance de nombreux retraités et retraitées internationaux nous a permis d'avoir un accès facilité à des personnes interviewées. On le voit, l'engagement de l'assistante sociale a été décisif à toutes les étapes du processus d'une recherche encore en cours. En tout cas, les premiers résultats montrent qu'une source importante de précarité est liée au décalage entre des vies professionnelles menées dans un cadre international (y compris au niveau des assurances vieillesse et maladie des fonctionnaires internationaux, qui dépendent des Nations Unies) et la nécessité ou le choix de s'insérer, au moment de la retraite, dans un cadre national avec d'autres règles, notamment en termes de protection sociale, qui peuvent exclure les anciennes et anciens fonctionnaires internationaux de certaines prestations sociales ${ }^{14}$ dépendantes des contributions à des caisses nationales.

Dans ces deux exemples, la recherche a été suscitée par des travailleuses sociales très engagées dans leur terrain d'intervention et préoccupées par des problématiques mettant en relation des questions d'âge et de 
migration. Leur dynamisme a interpellé les chercheur.e.s, a permis d'approfondir leurs interrogations initiales et de les co-construire sous forme de projets de recherche. Une fois les projets mis en œuvre, elles ont été partie intégrante de l'équipe de recherche et ont joué un rôle clé d'interface avec les instances et les populations directement concernées. La faisabilité de la recherche a été directement liée à ce rôle d'interface, qui facilita la communication entre les divers actrices et acteurs impliqués et permit de rendre visibles et clarifier certains enjeux liés à la problématique.

Ce double mouvement de visibilisation et de clarification peut aider les professionnel.le.s à mieux définir leur marge de manœuvre par rapport aux problématiques étudiées. Plus largement, il peut contribuer à penser autrement ces questions et soulever un débat sociétal plus large sur la manière habituelle de traiter ces problèmes avec une dimension transnationale. ${ }^{15}$ Cela est d'autant plus important que, souvent, les individus et les familles sont placés dans des situations particulièrement difficiles du fait du décalage entre leurs besoins et le cadre légal, organisationnel et habituel, prévu pour faire face à des difficultés rencontrées par les personnes âgées. Par ailleurs, dans les cas où une travailleuse sociale n'est pas associée directement à l'équipe de recherche, il faudrait imaginer, lors du processus de recherche et lors de la restitution des résultats, des manières de tenir compte de ce double mouvement de visibilitation et de clarification afin tionnaires internationaux retraités: le cas du Grand Genève» (Projet CEDIC 23-I2,
Claudio Bolzman), obtenu en 20I 2. Partenaire de terrain: Association des anciens fonctionnaires internationaux de Genève.

Cette région inclut le canton de Genève, la France voisine et une partie du canton de Vaud. Nombre d'anciennes et anciens fonctionnaires internationaux résident en effet dans la région.

Par exemple, le droit à recevoir un financement pour certaines prestations de l'aide à domicile ou lors de séjour en EMS.

Comme le montrent Wimmer et Glick-Schiller (2002), les questions transnationales sont souvent traitées à partir d'une perspective caractérisée par le nationalisme méthodologique. Cela veut dire que les frontières nationales constituent l'unité d'analyse des problèmes et que la notion de société est considérée comme identique à celle d'Etat-nation, confondant ainsi les intérêts nationaux avec la manière de penser les problèmes par les sciences sociales. Cette perspective dominante n'aide pas les personnes confrontées à des problématiques transnationales à trouver des solutions. 
que les questions spécifiques concernant les personnes migrantes puissent être prises en compte de manière pertinente par les services concernés.

\section{Remarques finales}

A travers l'exemple des recherches menées autour des questions relatives à l'âge et aux migrations, nous avons tenté de montrer que la spécificité de la recherche en lien avec le travail social se construit, ou du moins peut se construire, à travers le temps. Ici, nous avons mis en évidence que la recherche académique "classique» peut susciter des recherches mandatées et des recherches collaboratives. Parfois, l'ordre d'inscription dans le temps de ces trois types de recherche peut être différent. L'important est qu'elles contribuent à la fois à produire de nouvelles connaissances théoriques et empiriques sur des questions sociales, mais aussi de nouvelles réflexions sur les modes d'intervention des travailleuses et travailleurs sociaux, voire des pistes concrètes d'action face aux problématiques rencontrées.

Dans notre exemple, une recherche académique classique a permis d'attirer l'attention des actrices et acteurs institutionnels sur des problématiques délaissées par les institutions, car n'entrant pas dans le cadre de leur champ d'action ordinaire. Ce type de recherche a contribué à sortir la pensée des sentiers battus en rendant visible ce que les modes habituels de construire les problèmes sociaux empêchent de voir, et a donc permis aux actrices et acteurs institutionnels de se donner les moyens de redéfinir leur champ d'intervention.

Les recherches mandatées permettent en effet aux institutions de s'approprier une problématique peu claire en collaborant avec des chercheur.e.s. Dans le premier cas cité, les instances concernées sollicitent les chercheur.e.s pour mieux connaître ce qui est fait ailleurs par et pour les migrant.e.s âgé.e.s afin de mieux cerner ce qu'elles pourraient mettre en place dans leur sphère d'action. Dans le deuxième cas, il s'agit de mieux comprendre pourquoi la population immigrée âgée est peu présente dans les espaces d'animation socioculturelle prévus pour les personnes âgées et ce qui pourrait les inciter à y participer. La recherche peut contribuer de manière directe à répondre à ces demandes, tout en élargissant l'horizon de celles-ci pour le situer dans un cadre plus large de production de connaissances. Elle déploie ainsi son orientation vers l'application tout en restant en même temps fondamentale. 
La recherche peut aussi être initiée non par le haut des institutions, mais par «la base», à savoir les professionnel.le.s du travail social intervenant directement sur le terrain. Les exemples présentés dans cet article montrent qu'un dialogue fécond peut s'instaurer entre praticien.ne.s de l'intervention et praticien-ne-s de la recherche, conduisant à des recherches collaboratives. Celles-ci se distinguent par trois caractéristiques principales: une démarche de co-construction entre les partenaires concernés; le fait de jouer sur deux registres à la fois, celui de la production de connaissances et celui du développement professionnel des praticien.ne·s; la contribution de ces derniers au rapprochement, voire à la médiation entre communauté de recherche et communauté d'intervention (Desgagné, I997).

Dans les exemples présentés, les travailleuses sociales sont confrontées à des interrogations qu'elles souhaitent clarifier afin de soutenir leurs usagères et usagers de la manière la plus pertinente possible. La recherche peut fournir des éléments importants pour éclairer leur pratique, tout en mettant en évidence certaines problématiques qui dérivent d'un cadre contextuel plus large, telles que des dispositions légales ou administratives peu claires ou limitatives. En tout état de cause, la mise en place de telles collaborations entre praticien.ne.s de terrain et de la recherche est un processus qui nécessite des rapports de confiance qui se construisent avec le temps.

Quelles que soient les modalités de la recherche en lien avec le travail social dans le domaine de l'âge et des migrations, sa spécificité ne se situe pas dans le type des questions posées, ni dans les méthodologies utilisées. Elle apparaît dans le souci d'articulation avec les pratiques professionnelles. Ce souci, il ne faut pas cependant l'évaluer dans l'espace d'une recherche particulière, mais dans le cadre d'un processus qui peut s'étaler sur une période bien plus longue.

\section{Références bibliographiques}

Blanc, O. \& Gilliand, P. (Ed.). (I99I). Suisse 2000. Enjeux démographiques, Lausanne: Réalités sociales.

Bolzman, C. (I999). Le parcours de deux générations d'immigrés: un chemin d'intégration? Dans C. Bolzman \& J.-P. Tabin (Ed.), Populations immigrées: quelle insertion? Quel travail social? (pp. 4I-56). Genève et Lausanne: Editions ies et EESP.

Bolzman, C. (2008). Quelles articulations entre recherche et formation en travail social? L'exemple du domaine interculturel en Suisse romande. Dans J. Depireux \& A. Manço (Ed.), Formations d'adultes et interculturalité. Innovations en pays francophones (pp. I I5-I30). Paris: L'Harmattan. 
Bolzman, C. (20I4). Articuler recherche et travail social: l'exemple de la migration. Dans S. Voélin, M. Eser Davolio \& M. Lindenau, Le travail social entre résistance et innovation = Soziale Arbeit zwischen Widerstand und Innovation (pp.367-377). Genève et Lucerne: Editions ies et Interact Verlag.

Bolzman, C. (20I5). Personnes âgées, migrations et care. Enjeux intergénérationnels et pour les politiques sociales. Dans N. Hajji \& O. Lescarret (Ed.), Les mouvements sociaux à l'épreuve de l'interculturel (pp. I89-209). Paris: L'Harmattan.

Bolzman, C., Fibbi, R. \& Vial, M. (1996). La population âgée immigrée face à la retraite: problème social et problématique de recherche. Dans H. R. Wicker et al. (Ed.), Das Fremde in der Gesellschaft. Migration, Ethnizität und Staat (pp. I23-I42). Zurich: Seismo.

Bolzman, C., Fibbi, R. \& Vial, M. (I999). Les Italiens et les Espagnols proches de la retraite en Suisse: situation et projets d'avenir. Gérontologie et société, 9I, I37-I5I.

Bolzman, C., Fibbi, R. \& Vial, M. (200I). Les loisirs des personnes âgées immigrées: pratiques, besoins, demandes. [Rapport de recherche]. Genève: Institut d'études sociales.

Bolzman, C., Hirsch Durrett, E., Anderfuhren, S., Vuille, M. \& Jäggi M. (2008a). Le regroupement familial des ascendants: le traitement national d'une problématique transnationale. L'exemple de la Suisse. Retraite et Société, 55, 40-69.

Bolzman, C., Hirsch Durrett, E., Anderfuhren, S., Vuille, M. \& Jäggi, M. (2008b). Migration of Parents under Family Reunification Policies. A National Approach to a Transnational Problem. The case of Switzerland. Retraite et société, sélection 2008, 93-I2I.

Bolzman, C., Poncioni, R., Vial, M. \& Fibbi, R. (2004). Older labour migrants' wellbeing in Europe: the case of Switzerland. Ageing and Society, 24(3), 4I I-429.

Desgagné, S. (1997). Le concept de recherche collaborative: l'idée d'un rapprochement entre chercheurs universitaires et praticiens enseignants. Revue des sciences de l'éducation, 23(2), 37 I-393.

Fibbi, R., Bolzman, C. \& Vial, M. (I999). Age et Migration. Expériences européennes pour et par les migrants âgés. Zurich: Pro Senectute, Commission fédérale des étrangers.

Hoggart, R. (I970). La culture du pauvre. Paris: Minuit.

Jaeger, M. \& Mispelblom Beyer, F. (20I4). Introduction. Dans M. Jaeger (Ed.), Conférence de Consensus. Le travail social et la recherche (pp. I-5). Paris: Dunod.

Jäggi, M. (2004). Perceptions des directeurs des organisations cantonales et des assistants sociaux de Pro Senectute, face à la diversité culturelle de leurs clients et implications sur les pratiques professionnelles [Travail de certificat, CAS «Migrations et pratiques professionnelles»]. Lausanne et Genève: Université de Lausanne, EESP, HETS.

Lopes, F. (2006). Le voyage inachevé: la prise en charge des personnes âgées immigrées en EMS. [Mémoire de fin d'études]. Genève: Haute école de travail social.

Rapport de la Commission fédérale sur la vieillesse (1995). Vieillir en Suisse: Bilan et perspectives. Berne: CFV.

Righard, E. (2008). The welfare mobility dilemma. Transnational strategies and national structuring at crossroads. Lund: Media Tryck.

Sayad, A. (I99I). L'immigration ou les paradoxes de l'altérité. Bruxelles: De Boeck. 
43 | Quels liens entre recherche et travail social?

Stalder, J. (I993). Question ordinaire, 9.6.93: "40\% d'étrangers en l'an 2040? ». Sécurité sociale, 5,93 .

Vuille, M., Bolzman, C. \& Hirsch Durrett, E. (2013). Professional stances and personal values in the realm of transnational family reunification with older parents: social work practice in an emerging field. European Journal of Social Work, I6(3), 407-426.

Wimmer, A. \& Glick-Schiller, N. (2002). Methodological nationalism and beyond: nation-state building, migration and the social sciences. Global Networks, 2 (4), $301-334$. 



\section{La recherche dans les hautes écoles, entre "débat académique» et «débat citoyen"}

Reconnaissance et accompagnement de la mixité des approches

Monica Battaglini et Laurent Wicht

\section{Introduction}

Réaliser des recherches sur mandat d'actrices et d'acteurs impliqués dans l'action publique est une manière parmi d'autres d'exercer une activité de recherche en sciences sociales. Choisir une problématique et une approche, enquêter, analyser, synthétiser et communiquer des résultats sont autant d'actions communes à toute recherche, mais qui prennent une tournure différente selon les objectifs poursuivis à court terme. Même si l'on peut admettre que toute étude a comme finalité générale de servir la connaissance, et donc d'améliorer dans un certain sens la condition humaine, si on regarde les objectifs plus immédiats, on s'aperçoit que certaines recherches s'orientent prioritairement vers une compréhension/explication de la réalité et de ses mécanismes, alors que d'autres envisagent cette même démarche comme une première étape dans la préparation de l'action. Par la démarche scientifique, certaines recherches visent à comprendre et expliquer les phénomènes sociaux sans expliciter le lien avec l'utilisation potentielle d'un tel accroissement de la connaissance. Certes, les avancées produites par ces recherches pourront induire des effets plus tard, mais les modalités du passage de la connaissance à l'action ne sont pas structurées à l'avance. D'autres recherches explicitent dès le départ, comme objectif à court terme, l'utilisation des résultats comme fondement d'action. En résumé, de notre point de vue, plus qu'une «recherche» sur un plan générique, on observe des manières différentes d'envisager et de conduire des recherches, notamment en fonction des objectifs poursuivis à court terme, que ceux-ci relèvent de la seule connaissance ou de la connaissance en lien avec l'action. Or, à ces objectifs différents correspondent des manières différentes d'élaborer et de réaliser les étapes du 
travail de recherche. Ce constat de «mixité» dans les manières de concevoir l'acte de la recherche nous est suggéré d'abord par l'expérience que nous pouvons relater à partir de plusieurs mandats réalisés dans le cadre d'actions publiques locales, mais aussi de la conduite de recherches finalisées principalement à la compréhension de phénomènes sociétaux. L'exploration de l'histoire des recherches mandatées au sein de la Haute école de travail social que nous avons effectuée en 2015 confirme cette diversité de conceptions (Battaglini \& Rodari, 20I5).

De notre point de vue, le fait d'identifier divers types de recherche ne vise pas une ségrégation entre les conceptions de cette activité, et encore moins entre les chercheur.e.s eux-mêmes. Convaincus de la complémentarité des approches, nos ambitions se limitent à amener une manière de structurer la pensée autour de la notion de "mixité de conceptions de l'approche» pour essayer de sortir du débat animé, voire houleux, entre tenant.e.s d'une recherche académique "pure» et défenseur.e.s d'une recherche «impliquée», voire "appliquée». Bien que digne d'intérêt, ce débat ne constitue pas à nos yeux un moyen très fécond pour faire avancer la réflexion sur la recherche en sciences sociales en général, et dans les hautes écoles en particulier. Il ne sera donc pas ici le lieu d'un approfondissement de cette controverse. ${ }^{1}$ Ainsi, nous ne nous attacherons pas à une mise en parallèle des pratiques dans un but de classification/hiérarchisation. Nous allons plutôt nous atteler à une description des particularités de la recherche mandatée par des actrices et acteurs impliqués dans l'action publique sous l'angle des potentialités et contraintes pour la recherche, et plus généralement pour le développement de la connaissance. Certes, si on considère la recherche et, plus généralement, la science d'un point de vue plus global, il apparaît clairement difficile de la séparer de la réalité historique, sociale et politique dans laquelle elle s'exerce. La recherche influence son environnement et est influencée par celui-ci. Au cours de l'histoire, par exemple, pour des

[...] raisons extra-théoriques [...] certaines théories sont encouragées et d'autres écartées [...] Est ainsi rappelé un truisme fort connu quoique souvent galvaudé: les sciences sont bien des constructions terriennes, entièrement humaines, qui prennent naissance, se déplient et se défont au beau milieu de conditions socio-historiques données (Karsz, 2017: 256).

Ainsi, bien que toute recherche soit d'une certaine manière impliquée dans la société, dans ce contexte, nous allons situer les recherches par 
rapport à l'implication plus ou moins directe des mandant.e.s dans l'action publique. En nous concentrant sur les mandats qui visent plus directement l'action, nous essayerons de montrer comment les chercheur.e.s organisent leur travail dans le cadre des relations humaines complexes qui caractérisent ce type de recherche.

Après avoir présenté le contexte dans lequel notre réflexion a lieu, à savoir la recherche sur mandat de collectivités locales dans le cadre des hautes écoles spécialisées en Suisse, nous allons présenter quelques éléments génériques qui caractérisent la recherche sur mandat. Des éléments décrits dans la littérature, qui est traversée par les débats mentionnés cidessus et qui vont nous permettre de préparer le balisage de la présentation d'une expérience de ce type d'étude. Le récit de la recherche qui suit concerne un mandat effectué par notre équipe entre 2008 et 20 I I. Un mandat dans lequel nous avons été amené.e.s à réaliser un monitoring sociologique de l'implantation de nouveaux logements d'utilité publique dans un quartier de la commune de Carouge, à Genève. La notion de «mixité sociale» était au centre de ce mandat. La "mixité des conceptions de la recherche» est ici au centre de notre propos.

\section{La recherche sur mandat au niveau local: caractéristiques, potentialités et contraintes}

Pour caractériser les recherches mandatées par des actrices et acteurs impliqués dans l'action publique, l'élément central est à notre sens la complexité des relations. Des relations de natures diverses, qui exigent des chercheur.e.s des compétences multiples.

Tout d'abord, comme dans toute recherche, on est amené à établir une relation avec l'objet qu'on s'apprête à étudier. En se questionnant sur les faits sociétaux, on utilise des concepts analytiques ad hoc, empruntés à la littérature et/ou élaborés, pour construire un objet de recherche. La relation avec cet objet devient étroite. Il influence l'approche au fil du temps autant qu'il est influencé par elle. Peu importe si, d'un point de vue

1 Débat dont on trouve un aperçu, par exemple, dans les échanges entre Jacques Le Bohec (20I0) et Isabelle Garabuau-Moussaoui (20I I) à propos de l'ouvrage Les méthodes qualitatives (Alami et al., 2009). 
épistémologique, la démarche s'insère dans un paradigme explicatif ou compréhensif (Charmillot \& Dayer, 2007), la recherche s'intéresse à l'être humain sur un plan individuel et collectif dans le but ultime d'accroître la connaissance. Dans le cadre des recherches mandatées par des actrices et acteurs impliqués dans l'action publique, les thèmes recouvrent une dimension supplémentaire. Selon Olivier de Sardan (20I0), il existe une «valeur ajoutée» dans le fait d'être à la fois dans un «débat académique» (qu'il juge, comme nous par ailleurs, indispensable à toute recherche) et dans ce qu'il appelle un «débat citoyen». Ces objets de recherche ont ainsi une double caractéristique: non seulement ils sont intellectuellement intéressants, mais ils le sont aussi socialement et politiquement (Olivier de Sardan, 20Io).

La relation qu'on entretient avec ces objets est d'autant plus intense qu'ils représentent un intérêt pluriel pour la recherche et amènent à se positionner dans des réseaux multiples. Le premier réseau n'est pas spécifique à la recherche mandatée par les actrices et acteurs impliqués dans l'action publique. Il s'agit de l'ensemble des relations entre chercheur.e.s, à l'intérieur et à l'extérieur de l'équipe de recherche. Les collaborations, la concurrence entre plusieurs équipes, les questions de pluri/multidisciplinarité se posent dans ce contexte, constituent des enjeux majeurs de la recherche académique et influencent indéniablement les choix opérés dans diverses étapes du processus. A ce faisceau relationnel déjà passablement complexe, et lorsque le questionnement est initié par des actrices et acteurs impliqués dans l'action publique, s'ajoutent les relations au-delà du monde de la recherche: les mandant·e.s, les personnes impliquées dans le terrain d'enquête, la sphère politique... L'initiation du questionnement n'appartient pas exclusivement aux chercheur.e.s, dans ce contexte, mais aussi à ceux et celles qui mandatent l'étude et/ou qui bénéficient des résultats et qui n'ont pas nécessairement le même référentiel théorique ou les mêmes objectifs dans la construction de l'objet d'enquête. Il découle de cela une nécessité de négociation avec les chercheur·e.s impliqué.e.s et les mandant.e.s, et ce dès le début du processus, pour déterminer la pertinence du questionnement et son éventuelle réorientation.

Les chercheur.e.s interprètent la demande à la lumière de concepts analytiques et contribuent à déterminer l'objet en interagissant avec les mandant.e.s. La construction de l'objet de recherche est, dans ce contexte, une démarche collective mixte entre scientifiques et actrices et acteurs de terrain. Dans l'étude exploratoire que nous avons réalisée sur les mandats de recherche à la HETS (Battaglini \& Rodari, 20I5), l'expression «faire 
avec » était majoritairement employée par les chercheur·e·s interrogé·e·s pour décrire la plupart des étapes des études réalisées. L'interaction étroite avec les mandant.e.s fait partie intégrante de ce type de recherche, ce qui implique la nécessité de faire des compromis et requiert des capacités spécifiques qui vont bien au-delà des compétences scientifiques. Cela est d'autant plus saillant dans le contexte qui nous préoccupe ici, car les chercheur.e.s dont il est question opèrent dans un cadre institutionnel particulier qui est celui des hautes écoles, à savoir toutes les institutions de niveau tertiaire (en Suisse, les universités, les écoles polytechniques et les hautes écoles spécialisées). Des institutions ouvertes d'une manière générale sur la cité par vocation et par nécessité, car elles sont par essence en prise avec la réalité de leur environnement, tout d'abord en raison de leur fonction de formation. Cette tâche les amène, dans un certain sens, à un devoir moral de mise en lien avec les besoins de la société en connaissances et en compétences. L'enjeu principal se situe, à notre sens, dans l'interprétation de ces besoins et dans la manière d'y répondre. En clair, il ne s'agit pas d'une réponse mécanique de pédagogues et d'autres scientifiques aux demandes formulées par la cité, incarnée par les professionnel.le.s de terrain ou par les politiques, mais d'une mise en lien de ces demandes avec les connaissances préexistantes dans les hautes écoles dans un mouvement de co-construction explicite (lorsqu'il y a collaboration) ou implicite (lorsque les hautes écoles interprètent les besoins de la cité). Autrement dit, indépendamment des conceptions des un.e.s et des autres, on ne peut concevoir les hautes écoles comme étant hors de la cité. La mise en lien est une réalité qu'il est difficile de contester, tant les interactions sont étroites. Tout en défendant leur autonomie dans les choix des contenus, de la pédagogie et des méthodologies, les hautes écoles sont influencées dans leur action formatrice et de recherche par les tendances structurelles qu'on retrouve dans la société. Preuves en sont, par exemple, les réformes opérées dans les pays européens, que bon nombre de personnes s'accordent à définir comme une «marchandisation de l'enseignement supérieur» (Schultheis, Roca \& Cousin, 2008), tout à fait cohérente avec la diffusion de modalités managériales dans le fonctionnement de l'ensemble du secteur public (Giauque, 2003), du monde du travail et de la société dans son ensemble (de Gaulejac, 2005; Linhart, 20I5).

Dans ce contexte général de lien entre écoles et cité, le cas des hautes écoles spécialisées (HES) en Suisse est particulier, tant la proximité avec les milieux professionnels est élevée. Dans la deuxième moitié des années I990, 
la mise en place en Suisse d'un degré tertiaire dans la formation professionnelle a donné naissance aux hautes écoles spécialisées (HES) à partir des écoles professionnelles existantes. L'objectif de cette réforme était de donner à la Suisse un système de formation supérieure duale basée sur l'alternance entre lieux de formation et milieu professionnel (SER, 2006). La réforme de l'enseignement supérieur capitalise donc sur une tradition éprouvée de formation en alternance pour renforcer la formation professionnelle. Les HES naissent dans un contexte de valorisation des liens qu'elles entretiennent avec les milieux professionnels, et plus généralement avec la cité. Ces contacts ancrés et continus entre les actrices et acteurs de terrain et les écoles constituent donc, dès la naissance des HES, un canal privilégié de passage d'offre et de demande de connaissance qui s'entremêlent. Le lien entre écoles et environnement social est, dès le départ, partie intégrante de la mission qui est confiée aux HES. La recherche, souvent déjà présente dans les écoles professionnelles préexistantes, se développe au sein des HES tout naturellement dans ce contexte de relations étroites entre science et cité. Les archives des activités de recherche, à l'Institut d'études sociales (IES) d'abord et à la Haute école d'études sociales (HETS) ensuite, montrent des chercheur.e.s très inséré.e.s dans des réseaux locaux, réalisant à la fois des mandats de recherche, des formations, voire d'autres types de services (Battaglini \& Rodari, 20I5). L'insertion dans ce tissu relationnel dense et institué a eu, et a encore, un impact sur la manière de concevoir la recherche et engendre à la fois opportunités et difficultés. Un impact qu'on peut analyser à partir de la réflexion d'Olivier de Sardan (20I0). Ce dernier identifie dans la démarche mandatée par les instances publiques trois difficultés principales: la détermination des thématiques et des objets de recherche, les contraintes sur le questionnement et sur la méthodologie de recherche et, enfin, les possibilités d'application des résultats de recherche. En toile de fond, on entrevoit les risques encourus en raison des liens plus ou moins étroits entre, d'une part, les chercheur.e.s et, d'autre part, les professionnel.le·s impliqué.e.s dans l'action publique. Comme le rappellent Frauenfelder et Mottet (2012), une participation de la «science» à l'organisation de l'action publique n'est pas sans risques, tant le rôle double de savant-expert/savant-acteur est délicat à assumer entre "validation des problèmes publics», normativité et diffusion de notions au statut épistémologique flottant (Frauenfelder \& Mottet, 20I2). Des difficultés à mettre en regard avec les risques inhérents à une recherche orientée prioritairement vers la compréhension/explication 
de phénomènes, à savoir un défaut d'opérationnalisation de la connaissance. S'il est clair, à nos yeux, que les recherches qui visent à être une base pour l'action ne peuvent se passer de la recherche «fondamentale» - sous peine d'être instrumentalisées -, il se peut que cette dernière ne soit jamais à même d'être utilisée pour penser, actualiser, améliorer des pratiques (Olivier de Sardan, 2010), et de fait, finisse par fonctionner en vase clos. La connaissance ne serait alors utile qu'aux personnes qui peuvent la comprendre. Ce qui reviendrait à une vision très élitiste de la science.

Pour illustrer ces difficultés, mais aussi les opportunités que ces engagements représentent pour les scientifiques, nous proposons de revenir sur les principales étapes d'une recherche mandatée par l'Office du logement du canton de Genève que nous avons réalisée au sein de la HETS.

\section{Monitoring d'un programme de logements d'utilité publique: la mixité dans tous ses états}

En avril 2008, les autorités cantonales genevoises lancent, en collaboration avec deux fondations immobilières, ${ }^{2}$ un appel d'offre pour un monitoring sociologique d'un programme d'implantation de deux immeubles dans un quartier de la commune de Carouge: L'opération pilote HBM I99 Tambourine sur la mixité sociale. Le cahier des charges de cet appel d'offre jetait les bases d'une étude, sur trois ans, des effets de la mixité sociale dans le quartier de la Tambourine, dans lequel deux nouveaux immeubles avaient été construits dans le cadre de la loi pour la construction de logement d'utilité publique (LUP). ${ }^{3}$ Dans ces deux immeubles, une pondération plus importante des loyers que l'usage habituel avait été introduite,

2 Fondations immobilières de droit public (FIDP) et la Fondation Kammacher.

3 La loi pour la construction de logements d'utilité publique (I 406 - LUP) a été votée par le Grand Conseil sans opposition le 24 mai 2007 et est entrée en vigueur le 3 I juillet 2007. Elle a été modifiée le I4 mai 2009, en renforçant les objectifs et les moyens à disposition. (Loi pour la construction de logements d'utilité publique (LUP - I 4 06). Loi générale sur les zones de développement (LGZD) et son article $4 \mathrm{~A}$.)

La loi fixe pour objectif à l'Etat de constituer un parc pérenne de logements à caractère social à hauteur de $20 \%$ du parc locatif du canton. A terme, le contingent pourrait atteindre environ 35000 logements. Consulté le 30.03.2017 sur https://www.ge.ch/logement/logement-utilite-publique/loi-lup.asp 
permettant ainsi une mixité économique des revenus des locataires au sein même des immeubles. Au printemps 2008, notre équipe de recherche, basée à la HETS, répond à cet appel d'offre. C'est à l'automne de la même année que le projet est accepté par les mandant.e.s.

Dans l'appel d'offre, les mandant.e.s formulaient la demande d'un suivi de l'implantation d'immeubles qui représentaient un exemple très explicite de la volonté de promotion de ce que les politiques appellent la «mixité sociale» sur le territoire genevois. Cette demande entrait dans un contexte de mise en avant de cette vision positive d'un habitat urbain empreint de mixité sociale, vue comme un rempart contre la construction de "ghettos". En Suisse et à Genève en particulier, on conçoit la mixité sociale comme un idéal du "vivre ensemble» au niveau des immeubles d'habitation, du quartier ou plus largement de la commune (Herzog, 2005). Dans le sens commun, cette conception de la mixité sociale met en avant la cohabitation harmonieuse d'une pluralité d'individus et de familles intégrées, au sein d'un même territoire, à la même collectivité.

Cette vision helvétique de la mixité sociale s'oppose radicalement à une conception anglo-saxonne de l'intégration, qui favorise le développement de quartiers homogènes du point de vue des appartenances socioculturelles ou socio-économiques en visant l'intégration de collectivités toutes entières dans le corps social. Cette conception est souvent associée à une notion perçue de façon très péjorative en Suisse, la notion de "ghetto". Dès la réponse à l'appel d'offre, la démarche de l'équipe de recherche a été de remettre en question cette vision incantatoire de la mixité sociale vue comme allant de soi pour s'opposer à une homogénéité ghettoïsante.

\section{Conception de la mixité sociale à Genève}

A Genève, tout semble se passer comme si cette appréhension du ghetto était amplifiée par effet de résonance avec les constats effectués dans certains quartiers suburbains français, dans lesquels le repli communautaire (Ellyas, 2006; Boucher, 2007) les fait ressembler à des «quartiers d'exil». En France, cette notion de repli communautaire, donc de non-mixité de nationalités et de cultures, viendrait se surajouter à une autre forme d'homogénéité qu'ont toujours connue les quartiers suburbains français, à savoir une homogénéité du revenu de leurs habitant.e.s. Dubet \& Lapeyronnie (I992) montrent bien que les banlieues françaises sont passées d'une homogénéité liée à l'appartenance commune à la classe ouvrière (les «banlieues 
rouges " des années I950) à une homogénéité de bas revenus (banlieue pour les personnes en difficultés sociales), déliée de toute autre appartenance de classe sociale ou d'appartenance «ethnique». En nous référant à Wacquant (2006), qui compare la situation française à celle des Etats-Unis, l'on pourrait dire que les quartiers périurbains français se sont constitués autour d'une notion d'homogénéité de classe ou de revenus et, dans le même temps, d'une mixité nationale ou culturelle, alors que, à l'inverse, les quartiers américains se sont constitués autour de l'homogénéité ethnique du ghetto (au sein duquel il existe une mixité socio-économique et socioprofessionnelle). Dès lors, si, dans une société française clairement organisée en classes sociales, il apparaissait acceptable qu'il existe une certaine cohésion sociale organisée autour de valeurs ouvrières, par exemple dans les «banlieues rouges» des années I950, de nouvelles formes de cohésion sociale organisées autour de l'appartenance communautaire viennent perturber fortement la conception de l'idéal républicain.

En Suisse, et à Genève en particulier, ces phénomènes sociétaux ne se sont pas vraiment réalisés. Ainsi, ce n'est pas l'appartenance nationale ou ethnique qui a servi de guide pour l'organisation de la ville et de ses quartiers. L'homogénéité de classe des quartiers type «banlieues rouges» qui se seraient transformés par la suite en quartiers d'exil, par le départ massif des ouvrières et ouvriers accédant aux classes moyennes, n'a pas non plus eu lieu à Genève. Bien au contraire, il apparaît plutôt que la cohésion sociale dans les quartiers périurbains construits dans les années I960-70 s'est organisée dès le départ essentiellement autour de l'appartenance à une certaine «classe moyenne».

Quid, alors, de la mixité sociale si la cohésion qu'on a pu observer dans ces quartiers, sortes de villes nouvelles genevoises, semble être liée plutôt à un principe d'homogénéité qu'aux effets de la mixité? Cette question est d'autant plus intéressante pour notre propos que le cœur du mandat qui avait été donné à la HETS reposait sur ce présupposé de «nécessité évidente» de mixité sociale, basée sur une figure mythique du «ghetto » à éviter à tout prix. Or, comme nous le mentionnons ci-dessus, la réalité genevoise est particulière et ne ressemble en rien à celle de banlieues françaises. Si on prend l'exemple des quartiers périphériques de l'agglomération genevoise comme Meyrin ou les Avanchets dans les années I960-70, on trouve une population plutôt homogène du point de vue du niveau socio-économique, mais résolument mixte du point de vue des nationalités qui la composent (Bergier, 1965). De plus, de son côté, la cité des Avanchets représente un 
exemple de mixité économique avec deux tiers de logements subventionnés et un tiers de logements en loyer libre ou en PPE ${ }^{4}$ (Gros, 2004).

Les observations et les études qui concernent ces quartiers montrent une satisfaction générale des habitant.e.s, du moins jusqu'au milieu des années I990. Est relatée une qualité de vie exceptionnelle basée sur une sorte d'esprit de village où règnent interconnaissance avec ses voisins et convivialité (Maitre, I99I). Les données quantitatives, comme le très faible taux de rotation des habitant.e.s, par exemple dans le cas des Avanchets (Gros, 2004), confirment les déclarations des habitant.e.s. Cependant, il ressort aussi de ces études que les débuts n'ont pas été si faciles; la cohésion sociale n'allait pas de soi et ne s'est pas faite «comme par enchantement». Au moment de leur création, ces quartiers sont habités par des jeunes couples avec enfants, certes d'origines nationales diverses, mais qui, dans le contexte socio-économique genevois des années I960 à I980, peuvent accéder relativement aisément au mode de vie de la classe moyenne. De plus, comme le relève Bergier (I965), les habitant.e.s se retrouvent autour de revendications pour la mise en place de services comme des espaces commerciaux ou autres espaces de loisirs. Ainsi, l'absence d'équipements collectifs semble agir comme un puissant facteur de stimulation de la participation collective. La relative mixité socio-économique et socioculturelle de ces quartiers évolue dans un contexte de participation à la revendication de meilleurs équipements commerciaux et socioculturels en donnant lieu à la création d'un consensus sur un mode de vie relativement homogène.

Les premières difficultés, dans ces environnements mixtes créateurs de consensus sur le mode de vie, se font sentir dès le début des années I990. Selon Gros (2004), la transformation de ces quartiers, qui les conduit vers une relative marginalisation, a lieu en raison de l'arrivée de nouveaux et nouvelles habitant.e.s. Des habitant.e.s qui sont essentiellement des locataires, rencontrant des difficultés sociales liées à la crise économique et à une migration récente (notamment en raison de la guerre des Balkans). Cette nouvelle mixité génère auprès des ancien·ne·s habitant·e·s un sentiment d'insécurité lié à la crainte de voir leur environnement direct changer. L'arrivée de personnes en difficulté socio-économique représente une augmentation de la mixité sociale qui bouscule les habitudes des habitant.e.s de longue date, notamment en termes de mode de vie.

L'étude réalisée dans le quartier des Tours de Carouge en 2006 (Wicht \& Chuard, 2006) permet d'illustrer ce mécanisme. Les observations effectuées dans ce contexte ont mis en évidence une forte cohésion sociale entre 
les personnes établies depuis longtemps dans ce quartier. Cette cohésion sociale s'était constituée peu à peu, sur la base d'un sentiment d'appartenance à un même groupe social, et plus largement à la commune de Carouge, de l'adhésion à des valeurs comme l'effort personnel, de l'engagement dans le travail, ou encore du respect de règles tacites, mais fortes. L'ensemble des personnes rencontrées avait aussi le sentiment très clair que la qualité de vie au sein des Tours était en train de se dégrader peu à peu. Pour expliquer cela, les habitant.e.s mettaient en avant deux raisons: l'arrivée de nouveaux et nouvelles locataires (désignés comme «cas sociaux") ne respectant pas les règles de base qui étaient fortement valorisées par ceux et celles qui étaient déjà là (discrétion, politesse, propreté et respect des équipements communs) et la présence de jeunes dans les espaces communs (allées, coursives). Dans ce cas, les figures des personnes nouvellement arrivées et des jeunes cristallisaient une forme de sentiment d'insécurité des ancien·ne·s habitant·e·s, qui considéraient que leur mode de vie homogène et partagé par toutes et tous risquait de disparaître.

Mixité, une distance sociale à combler

Dans cette perspective, si la notion de mixité sociale se présente d'abord comme un choix politique fort, en tant que donnée de départ, elle a pour conséquence l'établissement d'une certaine distance sociale entre les différent.e.s habitant·e.s. Ceux-ci vont donc être amenés à construire un référentiel commun, plutôt homogène, une sorte de proximité sociale en termes de conception de la cohabitation, cela pour combler cette distance de départ. Chamboredon (I970; I97I) montre bien l'importance de la distance sociale ou, à l'inverse, de la proximité sociale dans la constitution d'un sentiment d'appartenance à un quartier de grands ensembles, qui génère, par essence, une situation de grande proximité spatiale. Pour Chamboredon, cette dimension de proximité sociale va déterminer la capacité d'un groupe d'habitant.e.s de s'indigner ou non du comportement de certain·e-s actrices et acteurs, de désigner ou non des outsiders et de réguler à l'interne ou, au contraire, de requérir des institutions (police, service

4 La PPE ou "propriété par étage» est une copropriété, à savoir une forme d'habitation où plusieurs personnes sontpropriétaires d'une certaine partie de cette habitation. Les copropriétaires ont l'usage exclusif sur leur logement. 
social) pour réguler les difficultés qu'il rencontre au quotidien. Elias (I965), dans une recherche classique, démontre lui aussi que cette proximité sociale à l'origine de la constitution d'une forte cohésion sociale entre certain.e.s habitant.e.s d'un quartier anglais n'est pas déterminée par leur origine culturelle ou nationale (ces personnes sont de nationalité anglaise), et n'est pas non plus fonction de l'appartenance à une couche sociale puisqu'il s'agit essentiellement d'ouvrières et d'ouvriers. Cette étude permet à Elias de mettre en évidence la notion d'ancienneté dans la constitution du sentiment d'appartenance du groupe qui se considère comme établi. Un groupe de personnes établies qui va entrer dans une logique de distinction et de discrimination à l'égard des personnes nouvellement arrivées, notamment en mettant en évidence des éléments liés à l'ordre, au savoir-vivre, à la propreté. Wimmer (2003), dans une étude suisse plus récente, arrive aux mêmes conclusions qu'Elias. Il met en évidence que les groupes d'habitant.e.s des quartiers qu'il a interrogés et qui se considèrent comme établis ne se constituent pas autour d'une logique d'appartenance culturelle, et regroupent par là aussi bien des personnes de nationalité suisse qu'italienne, espagnole ou encore tamoule. Pour Wimmer, la dichotomie normative développée par les personnes établies se construit autour de la capacité d'adhésion aux valeurs du "propre en ordre" (propreté, discrétion, être sans histoire, etc.).

En synthèse, les études précédemment menées ainsi que l'utilisation de la littérature spécialisée permettent à l'équipe de recherche de questionner la pertinence de la notion qui est au cœur du mandat: la mixité sociale. Cette remise en question de la notion permettra une réorientation de l'objet de recherche et son recentrage sur la mixité des conceptions des modes d'habiter et de leur éventuelle convergence vers un compromis permettant le «vivre ensemble». Dès les premières discussions, tout l'enjeu était de proposer une description, en vue d'une définition, de la mixité introduite par le projet immobilier. L'observation du processus éventuel de mise en place d'un mode de vie consensuel dans le quartier a été rajoutée à la recherche. C'est le rapport entre distance sociale génératrice potentielle de conflit et référentiel commun homogène permettant une cohabitation harmonieuse qui devient l'objet de la recherche. Paradoxalement, c'est la mixité introduite pour faciliter la cohabitation qui la rend plus difficile. Le but de l'action se déplace donc, le cas échéant, vers un accompagnement afin de mieux réguler le fonctionnement du «vivre ensemble» dans l'immeuble ou le quartier. 


\section{De la mixité sociale à la construction de la cohabitation: redéfinition d'un mandat}

La difficulté principale du processus de redéfinition du mandat était l'introduction d'une vision moins idéalisée, mais plus réaliste de la mixité sociale, qui devenait dans la démarche scientifique un obstacle à la cohabitation, alors qu'elle avait été introduite dans la politique d'aménagement urbain précisément pour faciliter cette cohabitation. Le glissement sémantique vers une notion de «distance sociale initiale» à la place de la «mixité sociale» a permis de sortir rapidement d'une idée de mesure de la satisfaction des habitant.e.s vis-à-vis d'une mixité mal définie. A la place a été réalisée une observation du processus de création éventuelle d'un référentiel commun de cohabitation pouvant servir de base à la mise en place d'une action d'accompagnement de la mixité introduite dans les immeubles.

La structure de recherche suivante a été proposée aux mandant.e.s et acceptée rapidement.

\begin{tabular}{|c|c|c|c|c|}
\hline $\begin{array}{l}\text { Axe } \\
\text { statique }\end{array}$ & $\begin{array}{l}\text { Axe } \\
\text { dynamique }\end{array}$ & $\begin{array}{l}\text { Axe } \\
\text { statique }\end{array}$ & $\begin{array}{l}\text { Axe } \\
\text { dynamique }\end{array}$ & $\begin{array}{l}\text { Axe } \\
\text { statique }\end{array}$ \\
\hline Temps 1 - & $\ldots$ & Temps 2 - & - & Temps 3 \\
\hline $\begin{array}{l}\text { Mesure de } \\
\text { la distance } \\
\text { sociale initiale }\end{array}$ & $\begin{array}{l}\text { Mise en } \\
\text { évidence, suivi } \\
\text { et qualification } \\
\text { des processus } \\
\text { de régulation } \\
\text { engagés entre } \\
\text { les différents } \\
\text { acteurs et } \\
\text { actrices }\end{array}$ & $\begin{array}{l}\text { Mesure de } \\
\text { l'évolution de } \\
\text { la distance } \\
\text { sociale, de la } \\
\text { constitution } \\
\text { d'un } \\
\text { référentiel } \\
\text { commun et } \\
\text { des effets des } \\
\text { régulations } \\
\text { engagées }\end{array}$ & $\begin{array}{l}\text { Mise en } \\
\text { évidence, suivi } \\
\text { et qualification } \\
\text { des processus } \\
\text { de régulation } \\
\text { engagés entre } \\
\text { les différents } \\
\text { acteurs et } \\
\text { actrices }\end{array}$ & $\begin{array}{l}\text { Mesure de } \\
\text { la distance } \\
\text { sociale finale, } \\
\text { de l'état du } \\
\text { référentiel } \\
\text { commun et } \\
\text { des effets des } \\
\text { régulations } \\
\text { engagées }\end{array}$ \\
\hline
\end{tabular}

Tableau I Plan de la recherche proposé au mandant par l'équipe

La redéfinition du questionnement s'est faite dès les premières séances entre l'équipe de recherche et les mandant·e.s. La problématique qui a résulté de ce processus a permis aux mandant·e.s et à l'équipe de recherche 
de trouver un intérêt commun dans le travail de monitoring proposé. En questionnant les premières demandes des mandant.e.s, il a été possible de trouver un compromis entre l'intérêt scientifique à accroître la connaissance dans la compréhension des phénomènes de construction de la cohabitation dans un quartier et l'intérêt politique à documenter les résultats d'une expérience pilote servant de base à d'autres réalisations éventuelles. Cela a pu être réalisé alors même que les référentiels d'action (introduction de la «mixité sociale» pour éviter le "ghetto») qui avaient été utilisés par les actrices et acteurs de terrain étaient remis en question par l'équipe de recherche. Il en a résulté une construction analytique qui a permis de tenir compte des acquis scientifiques du moment et des besoins en connaissance des actrices et acteurs de terrain.

Le contexte spatial de l'étude a aussi fait l'objet de négociations. Le mandat de départ demandait un rapport sur les effets de la mixité dans les immeubles en faisant abstraction de leur environnement. Il était évident pour l'équipe de recherche que l'étude ne pouvait contourner les variables contextuelles liées aux caractéristiques spécifiques du quartier. Bien qu'on ne puisse pas parler, dans le contexte genevois, de quartiers d'exil, comme nous l'avons montré ci-dessus, les possibilités de mobilité sur un plan spatial et social paraissent déterminantes dans la prévention de phénomènes de ségrégation (Wicht, Christe et al., 20I I). Des négociations supplémentaires ont été nécessaires pour permettre une analyse plus contextualisée. Tout en étant consciente des limites matérielles du mandat, notamment au niveau des ressources, l'équipe de recherche a demandé et obtenu un élargissement partiel de l'enquête. ${ }^{5}$ L'historique du fonctionnement du quartier dans lequel les immeubles sont implantés, sa topographie, sa démographie constituent des variables contextuelles primordiales dans la compréhension de l'évolution de la cohabitation. Dans ce contexte, cet élargissement était d'autant plus important en raison de la configuration territoriale, qui fait de ce quartier une réalité symboliquement et physiquement ${ }^{6}$ séparée du reste de la commune.

Les possibilités données par la mobilité physique (desserte en transports publics), l'accès à la formation, au travail, au logement et la qualité des aménagements extérieurs (espace public, lieux de rencontre) sont déterminants pour savoir si un quartier devient ou non un lieu d'exclusion sociale. L'intégration socioculturelle se fait en premier lieu dans des voisinages homogènes et non pas dans des voisinages hétérogènes (Schulte-Haller, 20 I : 6). 
Au fil des rapports entre mandant·ess et équipe de recherche, une complexification de la problématique a pu se déployer avec le passage d'un questionnement sur l'impact d'une certaine mixité sociale (économique) sur la cohabitation dans deux immeubles à l'étude de la dynamique de la mise en place du "vivre ensemble» dans un quartier, en partant de ces deux immeubles spécifiques. Ce processus a impliqué une remise en question de la part des mandant·e•s, qui ont implicitement accepté que soit intégrée dans la démarche l'étude des choix opérés dans la composition de la mixité des immeubles. Autrement dit, dans cette recherche, il a été possible d'inclure l'action des mandant.e.s dans l'objet d'étude. Un des biais potentiels de ce type de recherche, évoqués par Olivier de Sardan (2010), a pu être ici évité grâce à l'ouverture des mandant.e.s à une interaction prolongée avec l'équipe de recherche. La mise en place d'un "groupe de pilotage ", qui a structuré les interactions entre mandant·e.s et mandataires, a eu deux effets principaux. Tout d'abord, l'accès au terrain d'enquête a été facilité. Les contacts sur le périmètre d'étude ont été aisés et les données collectées riches et de bonne qualité. Ensuite, les contacts formels et informels entre mandant.e.s et mandataires ont favorisé un "phénomène d'apprentissage " semblable à celui décrit dans une partie de la littérature sur l'analyse de politiques publiques (Knoepfel, Larrue \& Varone, 2006; Sabatier \& Jenkins-Smith, I999). Dans ces approches, qui traitent tout particulièrement des dynamiques de changement au sein des politiques publiques, le propos est de mettre en avant les interactions entre actrices et acteurs comme base de l'évolution progressive des conceptions des dispositifs d'action. Les agent.e.s internes à l'action publique et les externes (expert.e.s ou autres) donnent l'impulsion à l'évolution dans la conception de l'action, et donc potentiellement à des changements. Certes, il ne s'agit pas ici de la mise en place d'une politique publique, mais d'une opération pilote dans le cadre d'une politique; aussi, un certain phénomène d'apprentissage a-t-il pu être observé. Le produit de ces interactions a été l'évolution de la vision de la mixité sociale au sein du groupe de pilotage formé par les représentant.e.s des mandant.e.s. Au cours des trois ans d'étude, une

5 L'équipe de recherche a proposé de réaliser une série d'entretiens et d'observations dans le quartier, bien que la démarche principale à laquelle était dédiée la plus grande partie de ressources fût centrée sur lesdits immeubles.

6 Le quartier de la Tambourine est implanté en périphérie de la commune de Carouge, commune genevoise de 20000 habitants. 
vision plus nuancée de l'impact de la mixité sociale sur le quartier a été construite au sein du groupe de pilotage.

La concertation qui a eu lieu dès le début de la recherche a permis aussi de réduire les risques potentiels liés aux possibilités de contrainte des mandant.e.s sur les choix méthodologiques, comme l'évoque Olivier de Sardan (20Io). Dès le départ, il a été proposé aux mandant.e.s une collecte de données sur une base mixte combinant approche quantitative et qualitative. Un questionnaire standardisé et des entretiens qualitatifs ainsi que des observations continues des immeubles et du quartier ont permis de réunir les données nécessaires au suivi de l'évolution du projet.

L'utilisation d'une méthodologie de collecte de données mixte constitue un avantage sur un plan scientifique. Selon le principe de la triangulation des sources de données (Yin, I994: 92), multiplier les techniques d'enquête en faisant appel tour à tour à des sources différentes permet de minimiser les biais propres à chacune des méthodes de collecte d'informations. De cette façon, la même information peut être collectée plusieurs fois avec des techniques différentes, ce qui augmente sa fiabilité. Sur le plan des relations entre mandant.e.s et équipe de recherche, ce type de construction méthodologique permet de mieux tenir compte des besoins des un.e.s et des autres. En cohérence avec les méthodes de gestion en vigueur depuis les années I990, la demande en données quantitatives est bien présente dans les mandats d'actrices et acteurs impliqués dans l'action publique. Il faut rappeler que ces actrices et acteurs sont issus pour l'essentiel des secteurs public ou parapublic. Les services de l'Etat et leurs partenaires ont adopté pour la plupart les principes de la nouvelle gestion publique (NGP), inspirés par la littérature américaine et remontant à l'ouvrage Reinventing government (Osborne \& Gaebler, I993). Ces méthodes managériales font la part belle aux indicateurs quantitatifs et ont favorisé le développement d'une culture administrative privilégiant des données quantitatives, perçues comme plus faciles à comparer et à communiquer (Giauque, 2003; Giauque \& Emery, 2008). Prévoir une approche mixte (quantitative et qualitative) permet donc de répondre aux exigences de simplicité dans la présentation des résultats tout en maintenant la richesse et la fiabilité de l'approche. Ces choix méthodologiques ont été possibles en raison de la marge de manœuvre que l'équipe de recherche a pu maintenir dans la démarche. Le récit du développement de ce mandat montre une équipe de recherche relativement autonome vis-à-vis des mandant.e.s et capable d'animer une co-construction de la problématique des choix 
6 I La recherche dans les hautes écoles, entre «débat académique » et "débat citoyen»

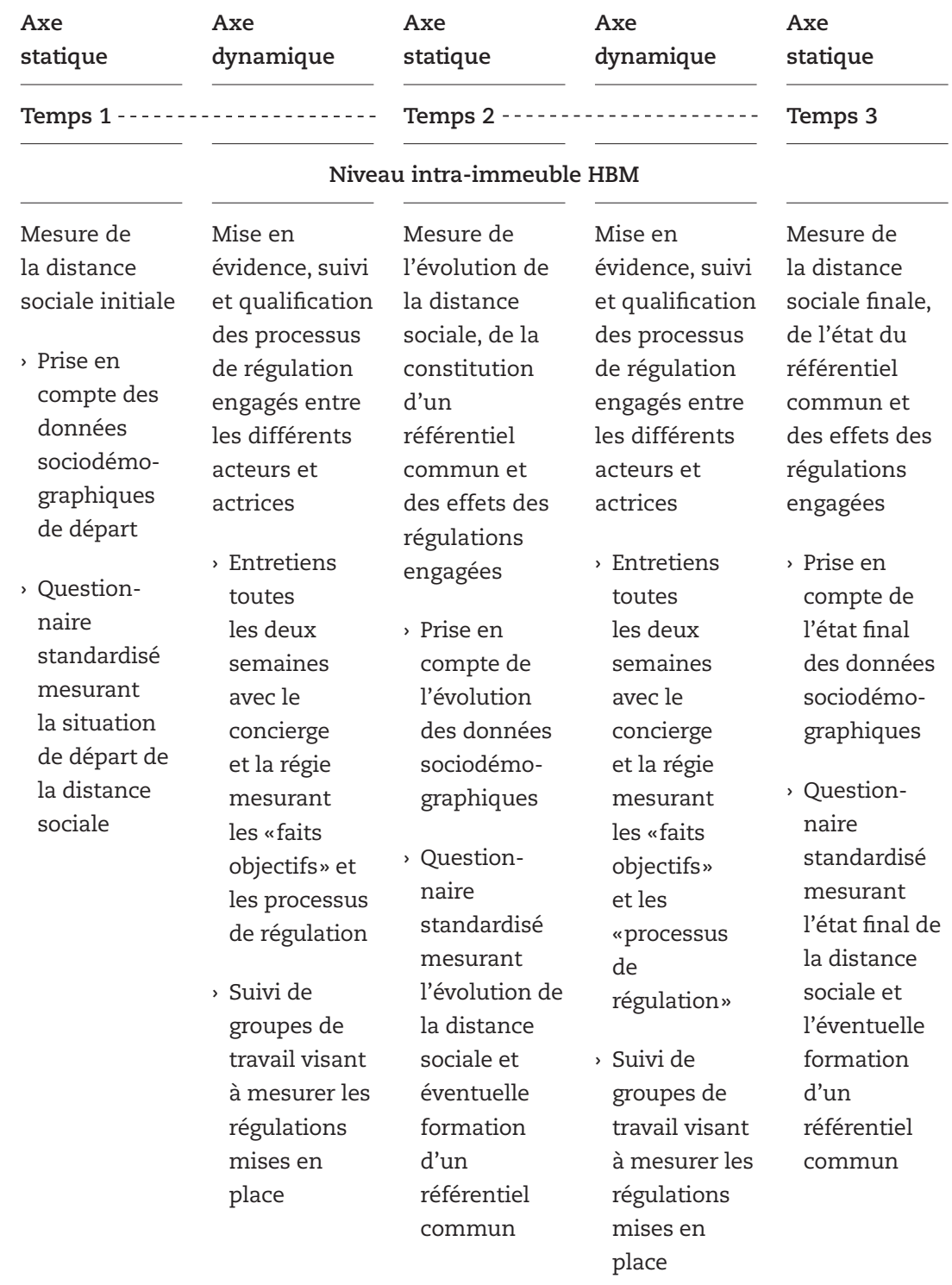

$\overline{\text { Tableau } 2}$ Structure de l'appareil méthodologique mis en place pour la recherche 
méthodologiques en partant de présupposés scientifiques. Nous l'évoquions ci-dessus, les difficultés et les risques inhérents à ce processus ne manquent pas. Or, ici, le déroulement de l'action de recherche qui aboutit au maintien de l'autonomie des chercheur.e.s s'explique d'abord par la structuration de la relation de type contractuel qui a défini dès le départ les bornes de la liberté académique, basée sur un respect mutuel entre la haute école et les mandant·e.s. De plus, une partie du financement a été assurée par la haute école elle-même, via des fonds de recherche internes ainsi que par la mise à disposition de temps de travail de chercheur.e.s. La participation de fonds de recherche au financement constitue en soi un élément central de la qualité scientifique du processus de recherche. L'évaluation par les pairs permet un contrôle de la qualité scientifique de la démarche et fournit une certaine protection aux chercheur·e·s face à la pression qui pourrait être exercée par les mandant.e.s dans la construction de l'objet de recherche et dans les choix méthodologiques. En d'autres termes, la structuration institutionnelle et la mixité des financements permettent d'équilibrer le pouvoir entre mandant·e.s et mandataires, les mettant dans une situation partenariale plutôt que de subordination.

Cette structure relationnelle relativement horizontale entre mandant.e.s et mandataires a eu comme effet de rendre possible une valorisation multiple des résultats. Sur un plan scientifique, l'étude a été présentée dans divers lieux (Aifris, 20I I ; journée cantonale du logement, 20I2) et a contribué à l'avancement de la compréhension du «faire quartier» dans un contexte de distance sociale. Du côté de la formation, l'étude a été utilisée dans le cadre de la formation de base et de la formation continue à la HETS; elle a permis la diffusion d'une vision de la mixité sociale plus proche des réalités locales auprès des futurs travailleuses et travailleurs sociaux, potentiels utilisateurs et utilisatrices de ces notions en tant que metteur.e.s en œuvre des dispositifs d'intervention sociale dans les quartiers. Au niveau professionnel, la diffusion des conclusions de l'étude a engendré un changement de perception de la mixité sociale comme instrument de l'aménagement urbain. Dans le mandat, on trouve une conception mythique de la mixité définie par la diversité des revenus, garantissant la non-stigmatisation des locataires à faible capacité socio-économique et prévenant les dégâts à la propriété. Dans les présentations qui suivent l'étude, on commente une définition de la mixité sociale ayant

[...] trop souvent été présentée comme un remède miracle ou un vœu pieux. La réalisation d'une cohésion sociale demande un savoir-faire et des moyens, 
63 | La recherche dans les hautes écoles, entre "débat académique et "débat citoyen »

afin que l'expérience urbaine puisse être partagée équitablement entre tous et que la ville soit accessible aux usages pluriels. Elle doit trouver une cohérence d'échelle et de décisions en s'appuyant sur l'expérience des communes qui ancrent leurs actions dans la réalité des quartiers. ${ }^{7}$

Après trois ans d'étude, il a été possible de formuler un certain nombre de suggestions aux mandant.e.s pour soutenir la construction d'un «vivre ensemble» harmonieux dans les nouveaux immeubles, et plus généralement dans le quartier de la Tambourine. Synthétiquement, ces propositions allaient dans le sens d'une prise en compte des difficultés inhérentes aux différences et d'un accompagnement de cette mixité. En se référant à la vision de Donzelot (2006), qui affirme qu'

un quartier n'existe que si on le produit, il a été suggéré de travailler à l'identification des composantes sociales et ethniques sur un quartier, de ses forces associatives, mais aussi des questions qui unissent les gens comme celles qui les opposent. Ceci dans le but de les réunir autour d'initiatives susceptibles de faire prévaloir d'abord une tolérance mutuelle, ensuite un certain nombre d'objectifs communs (Donzelot, 2006: I56).

Le processus complexe de construction d'un «référentiel commun » d'interprétation de la réalité de la vie des immeubles et du quartier de la Tambourine, réalisé par les mandant.e.s et l'équipe de recherche, a débouché sur une conception proactive des actions à envisager dans ce quartier. La notion de mixité sociale a ainsi pu évoluer d'une perception idéalisée et peu opérante vers celle d'un outil d'analyse à mobiliser dans le cadre d'une agentivité performante, dans le but de construire une cohabitation harmonieuse dans un quartier.

\section{Conclusion: reconnaître et accompagner la mixité des approches}

A la lumière de ces expériences, nous pouvons retenir que le travail de recherche dans le cadre des recherches mandatées par des actrices et acteurs impliqués dans l'action publique comporte des risques et des

7 Extrait de la présentation des conclusions de l'étude par Laurent Wicht, lors de la journée du logement 2012. 
opportunités. Coté risques, nous retenons principalement les possibilités d'instrumentalisation de la connaissance, qui sont en lien avec la pression que les mandant.e.s peuvent exercer dans leur position de financeurs. Des risques à mettre en parallèle avec les recherches qui n'explicitent pas les liens avec l'action et qui, par une vision très élitiste de la connaissance, pourraient servir uniquement le cercle fermé de celles et ceux qui peuvent la comprendre. L'exemple du mandat effectué à Carouge montre que l'accompagnement institutionnel permet en grande partie de limiter ce double risque tout en saisissant l'opportunité de mettre en adéquation les conceptions des mandant.e.s avec les acquis scientifiques. La présence d'un financement mixte comprenant un fonds de recherche à côté de la rémunération par les mandant.e.s nous paraît tout particulièrement importante. La présence d'un fonds scientifique permet l'évaluation par les pairs, gage de rigueur, ainsi qu'une certaine autonomie des chercheur·e•s, fondée sur une marge de manœuvre financière. La mise en place de contrats qui formalisent les relations entre mandant.e.s et mandataires représente aussi un élément important, de par la clarification des rôles et des attentes. Dans notre exemple, cette structuration couplée à la mise en place d'un groupe de pilotage a généré un phénomène d'apprentissage permettant l'évolution d'une conception incantatoire de la «mixité sociale» vers une vision dynamique de la création d'un "vivre ensemble harmonieux». Dans ce contexte, les chercheur.e.s ont servi de pont entre des connaissances scientifiques en quête d'approfondissement empirique et une réalité sociale demandeuse de ces connaissances. Dans cette conception des processus, la relation est au centre des préoccupations; sa structure est primordiale pour que les divers acteurs et actrices y trouvent leur compte.

Ce travail de structuration des relations est possible s'il existe une reconnaissance institutionnelle de la diversité des manières de concevoir la recherche dans toutes ses étapes, de la construction de l'objet à la diffusion des résultats. Synthétiquement, il s'agit de mettre en avant les complémentarités et non la concurrence entre modalités de recherche afin de garantir les meilleurs échanges entre science et cité. A l'image des quartiers mixtes, il s'agit de reconnaître et accompagner la diversité d'approche dans la recherche pour minimiser les contraintes et maximiser les potentialités afin d'accroître à la fois la connaissance et son utilisation. 
65 | La recherche dans les hautes écoles, entre "débat académique » et "débat citoyen»

\section{Références bibliographiques}

Alami, S., Desjeux, D. \& Garabuau-Moussaoui, I. (2009). Les méthodes qualitatives. Paris: Presses Universitaires de France.

Battaglini, M. \& Rodari, S. (2015). Recension et analyse des mandats. [Rapport de recherche]. Genève: Haute école de travail social.

Bergier, J.-F. (I965). Meyrin-Genève. Revue de médecine préventive, V, 517-529.

Boucher, M. (2007). Turbulences: comprendre les désordres urbains et leur régulation. Montreuil: Aux lieux d'être.

Chamboredon, J.-C. (I97I). La délinquance juvénile, essai de construction de l'objet. Reune française de sociologie, I 2, 335-377.

Chamboredon, J.-C. \& Lemaire, M. (I970). Proximité spatiale et distance sociale. Les grands ensembles et leur peuplement, Revue française de sociologie, I I, 3-33.

Charmillot, M. \& Dayer, C. (2007). Démarche compréhensive et méthodes qualitatives: clarifications épistémologiques. Recherches qualitatives, hors série, 3. Consulté le 09.04.I7 sur http://www.recherche-qualitative.qc.ca/documents/files/ revue/hors_serie/hors_serie_v3/Charmillot_et_Dayer-FINAL2.pdf

de Gaulejac, V. (2005). La Société malade de la gestion. Idéologie gestionnaire, pouvoir managérial et harcèlement social. Paris: Le Seuil.

Donzelot, J. (2006). Quand la ville se défait. Paris: Seuil.

Dubet, F. \& Lapeyronnie, D. (I992). Les quartiers d'exil. Paris: Seuil.

Elias, N. (1965). Logiques d'exclusion. Paris: Pocket.

Ellyas, A.-B. (2006). Replis communautaires à Sarcelles. Manière de voir, Io(89), 46-48.

Frauenfelder, A. \& Mottet, G. (20I2). La fabrique d'un problème public. Reconnaître, expertiser et gérer la «violence en milieu scolaire». Revue suisse de sociologie, 38(3), 44I-457.

Garabuau-Moussaoui, I. (2010). «Les méthodes qualitatives: une réponse à Jacques Le Bohec", Lectures [en ligne]. (20I I, I2 mai). Consulté le I6.02.20I7 sur http://lectures.revues.org/5454

Giauque, D. (2003). Nouvelle gestion publique et régulation organisationnelle. La bureaucratie libérale. Reune Internationale des Sciences Administratives, 69(4), 663-692.

Giauque, D. \& Emery, Y. (2008), Repenser la gestion publique: bilan et perspectives en Suisse. Lausanne: Presses polytechniques et universitaires romandes.

Gros, D. (2004). Un exemple de marginalisation en zone urbaine. L'éducation en débat, 2, 3-2I.

Herzog, S. (2005). La Suisse ne connaît pas de ghetto social. Repère social, 70, 5. Karsz, S. (20I7), Affaires sociales, questions intimes. Malakoff: Dunod.

Knoepfel, P., Larrue, C. \& Varone, F. (2006). Analyse et pilotage des politiques publiques. Zurich: Rüegger.

Le Bohec, J. (20I0). «Sophie Alami, Dominique Desjeux, Isabelle GarabuauMoussaoui, Les méthodes qualitatives», Lectures [En ligne]. (2010, 5 octobre). Consulté le I6.02.20I7 sur http://lectures.revues.org/ I 46

Linhart, D. (20I5). La comédie humaine du travail. De la déshumanisation taylorienne à la sur-humanisation managériale. Toulouse: Erès. 
Maitre, M. (I99I). Meyrin: au-delà des grands ensembles. Dossiers publics, 79(28), 28-49.

Olivier de Sardan, J.-P. (20I0). Anthropologie médicale et socio-anthropologie des actions publiques. Anthropologie et santé, I. Consulté le I2.03.2018 sur http://journals.openedition.org/anthropologiesante/86

Sabatier, P. \& Jenkins-Smith, H., (1999). The Advocacy Coalition Framework: An Assessment. Dans P. Sabatier (Dir.), Theories of the Policy Process (pp. I I7-166). Boulder: Westview Press.

Schulte-Haller, M. (20I I). Mixité sociale et développement de quartier: entre désir et réalité. Berne: ARE, OFL, SEM, OFSPO, SLR, CFM

Schultheis, F., Roca i Escoda, M. \& Cousin, P.-F. (Dir.) (2008). Le cauchemar de Humboldt. Les réformes de l'enseignement supérieur européen. Paris: Raisons d'agir.

Secrétariat d'Etat à l'éducation et à la recherche. (2006). Panorama de l'enseignement supérieur en Suisse. Berne: SER.

Wacquant, L. (2006). Parias urbains. Paris: La Découverte.

Wicht, L., Christe, E., Battaglini, M. \& Chuard, C. (20I I). Faire l'expérience de la mixité sociale dans son quartier. [Rapport de recherche]. Genève: Haute école de travail social.

Wicht, L., Chuard, C. \& Seiler, I. (2006). Recherche-action participative au sein des Tours de Carouge. Habitants établis et outsiders: nuisances et qualité de vie dans le quartier. [Rapport de recherche]. Genève: Haute école de travail social.

Wimmer, A. (2003). Etrangers établis et outsiders indigènes, formation de catégories sociales et réseaux de relations dans trois quartiers d'immigrants. Dans H.-R. Wicker, R. Fibbi \& W. Haug (Ed.), Les migrations et la Suisse (pp. 197-226). Zurich/Genève: Seismo.

Yin, R. K. (1994). Case Study research. London: Sage. 


\section{Enquêter en «terrain difficile»}

Production et réception d'une enquête sociologique

dans un centre éducatif fermé

\section{Arnaud Frauenfelder, Géraldine Bugnon et Eva Nada}

Depuis les années 2000, les centres éducatifs fermés (CEF) font l'objet en Suisse, comme dans d'autres pays d'Europe, de dynamiques réformatrices d'apparence contradictoire. D'une part, la diffusion et la légitimité croissantes des droits de l'enfant contraignent ces institutions à «humaniser» le traitement des jeunes détenu.e.s et à les reconnaître comme sujets de droit. D'autre part, la montée des préoccupations sécuritaires en Europe envers la délinquance juvénile entraîne un renforcement des aménagements architecturaux sécuritaires et un durcissement des sanctions disciplinaires. Le souci d'offrir une prise en charge appropriée des mineur.e.s en détention, par des formes d'encadrement pluridisciplinaires et orientées autour de la promotion d'un régime de droit commun en détention (Sallée, 2012) se situe au cœur de la réforme pénale, questionnant le sens même de l'institution carcérale: une institution désormais soucieuse de devenir encore plus «humaine» tout en demeurant un espace de privation de liberté parfois renforcé (Fernandez, 2013; Bouagga, 2013). A partir d'une enquête de terrain réalisée dans un CEF de Suisse romande (le «Verger»), ${ }^{1}$ nous avons pu explorer cette ambivalence sociétale qui traverse la question de l'enfermement des mineur.e.s saisis comme sujets pénaux. Il s'agissait alors de pénétrer dans ce monde méconnu en donnant à voir les manières diverses, et parfois concurrentes, dont les différents groupes professionnels s'approprient cet espace et confèrent une raison d'être à l'enfermement de mineur.e.s. A partir de l'analyse, tout d'abord des rivalités

1 Pour préserver l'anonymat du terrain d'enquête, nous invisibilisons l'institution concernée. Dans cette logique, les termes génériques de CEF ou plus nominatif du «Verger», utilisés dans le texte pour désigner l'établissement concerné, sont fictifs. 
de territoires opposant groupes installés, corps éducatif, maîtresses et maîtres socio-professionnels, et nouveaux entrants, agent.e.s de détention et corps médical, puis des conceptions éducatives diverses qui sont engagées dans leurs pratiques et, enfin, du rapport à l'environnement extérieur, nous nous sommes attaché.e.s à saisir les formes de recomposition de l'économie morale de l'enfermement ${ }^{2}$ en CEF. Au final, l'analyse sociologique de la justice pénale des mineur.e.s "par le bas" permet de découvrir combien les pratiques de l'Etat, dans cet univers particulier du monde social, s'expriment d'abord par le travail de ses agent.e.s.

Plutôt que de présenter les résultats de cette recherche (Frauenfelder, Bugnon \& Nada, 2015a; Frauenfelder, Nada \& Bugnon, 2015b; Frauenfelder, Nada \& Bugnon, 20I8), cet article entend revenir sur les conditions de production ${ }^{3}$ et de réception de cette enquête sociologique en "terrain difficile" (Boumaza \& Campana, 2007), en montrant combien l'analyse de celles-ci - qui sont généralement peu analysées en tant que telles - peut contribuer à la construction de l'objet étudié, en mettant sur une piste de recherche féconde, ainsi qu'à une mise à l'épreuve des interprétations issues de l'analyse des données empiriques. Plus qu'un simple exercice de style de type scolastique ou d'une "preuve" d'adhésion à une certaine éthique de la recherche (pratiques collaboratives avec les personnes enquêtées, impératif de restitution des résultats), l'examen de ces "coulisses» de la production et de la réception d'une recherche en CEF représente une exigence scientifique de réflexivité qui joue un rôle actif dans la production de connaissances en sciences sociales (Becker, 2002; Beaud \& Weber, 2003; Bourdieu \& Wacquant, 20I4).

Notre démonstration s'organise en deux parties. La première rend compte des conditions de production de la recherche en s'intéressant aux différents types de difficultés rencontrées: celles propres au terrain d'enquête du CEF, qu'elles viennent des pressions des autorités fédérales de justice, des critiques médiatiques dont l'institution pouvait être la cible, ou celles liées à notre position de chercheur.e.s en sciences sociales mettant les pieds dans un univers déjà pensé par des savoirs d'expertise. Nous concluons cette partie en montrant comment les difficultés relatives à cette situation d'enquête ont participé à la construction de notre problématique sociologique. La deuxième partie de cet article aborde les conditions de réception de la recherche par les différents corps professionnels du CEF, qui ont participé à la mise à l'épreuve, et la consolidation de nos résultats. 


\section{Une situation d'enquête difficile}

Si n'importe quel terrain est susceptible d'être considéré comme difficile, «certains le sont certainement plus que d'autres» (Boumaza \& Campana, 2007: 8). L'univers carcéral représente un exemple paradigmatique à cet égard (Bouagaga, 2013). Il est particulièrement contrôlé, plusieurs groupes professionnels y coexistent et il est particulièrement sensible au regard extérieur. Le CEF étudié - le «Verger» - présente la particularité d'être destiné à des séjours de courte durée, ne dépassant généralement pas trois mois. Trois autres établissements de ce type existent en Suisse, alors que les onze autres CEF situés sur le territoire sont réservés à des séjours de moyenne ou de longue durée, de dix-douze mois à trois-quatre ans (OFJ, 20I3: 6-7). Notons, enfin, que les CEF demeurent minoritaires parmi l'ensemble des établissements d'éducation pour mineur.e.s reconnus par l'Office fédéral de la justice. Sur les I8o établissements reconnus par l'OFJ, seuls I5 disposent d'une section fermée, soit $8 \%$. En Suisse, effet de fédéralisme oblige, les CEF sont gérés selon des modalités variables selon les cantons $^{4}$ : bien souvent, ils dépendent d'une gestion orchestrée par des instances concordataires intercantonales ${ }^{5}$ chargées de la détention pénale des mineur·e•s, d'offices cantonaux spécifiques chargés de l'administration de la détention, alors que leur mandat spécifique vise l'exécution, par un personnel qualifié, de décisions prises par les tribunaux de mineurs. Si l'on trouve des CEF dans différents pays d'Europe, en France, en Belgique

2 Le concept d'économie morale peut se définir, selon Fassin et al. (2013: 23), comme "la production, la circulation et l'appropriation des valeurs et affects dans un espace social donné. Elles caractérisent donc, pour un moment historique particulier et un monde social spécifique, la manière dont est constituée une question de société à travers des jugements et des sentiments qui définissent ainsi progressivement une sorte de sens commun et d'appréhension collective du problème. »

3 En s'inspirant d'un article publié en 2014 dans Cultures et sociétés (Frauenfelder, Nada \& Bugnon, 20I4).

4 Même si la nouvelle loi fédérale sur la procédure pénale unifiée des mineurs (PPmin), entrée en vigueur en 20I I, entend harmoniser les différentes procédures cantonales.

5 A l'image de la création en 2005 du Concordat romand des institutions de détention pour mineurs. 
ou encore en Suisse, la réalité que recouvre cette appellation est hétérogène du fait des spécificités des espaces considérés, de l'histoire singulière des institutions, des métiers ou encore des bâtiments concernés. A bien des égards, les textes circulent sans les contextes. Ainsi, en France, la fermeture du CEF est plus juridique que réelle (Sallée, 20ıо; 2013; Lenzi \& Milburn, 2015: 97-98), ${ }^{6}$ ce qui n'est pas le cas en Suisse, où les aménagements externes, grillages, barbelés et caméras de surveillance, symbolisent bien le caractère de confinement du lieu. Au-delà des variations contextuelles, les CEF semblent avoir pour caractéristiques principales d'être des institutions hybrides. Elles se définissent d'abord par ce qu'elles ne sont pas. Au sens strict, elles ne sont ni des prisons ni des foyers éducatifs. Plus positivement, les CEF «constituent plutôt un espace d'enfermement hybride destiné aux mineurs placés pour faits délictueux, soumis à la double contrainte de la restriction de leurs libertés dans le présent et de l'obligation de participation à des activités pour préparer leur avenir» (Lenzi \& Milburn, 20I5: 96). Si l'univers social du CEF étudié demeure un espace particulièrement contrôlé, pouvoir y accéder ne va pas de soi. L'entrée dans le terrain s'avérait particulièrement difficile en raison du contexte de «crise» à laquelle l'institution était exposée, même si cette situation était aussi à l'origine de notre venue.

Une institution marquée par un contexte de «crise»

L'enquête au "Verger» a été réalisée dans un contexte de crise institutionnelle: le concept d'intervention proposé par la direction de l'établissement - qui réunit les aspects centraux du fonctionnement et des objectifs poursuivis par l'institution - avait en effet été invalidé par l'instance fédérale chargée de son financement. Conséquence de la réforme pénale engagée, cette situation a également été observée dans d'autres établissements d'éducation fermés en Suisse ne répondant pas suffisamment aux standards du nouveau DPmin (OFJ, 2007; 20I3). Lors d'une première rencontre en janvier 20I I avec les professionnel-le.s et la direction du «Verger», face à la complexité des réalités thématisées par les professionnel.le·s présents, nous avons proposé de mener une étude approfondie sur ce champ d'intervention professionnelle afin de comprendre les transformations diverses qui le traversaient. En tant que chercheur.e.s en sciences sociales, pouvoir entrer dans un terrain d'enquête peu accessible est, bien sûr, intellectuellement très stimulant. Bien que le développement récent, en France, de diverses 
structures «contenantes" pour mineur.e.s amène les sciences sociales à s’y intéresser (Mucchielli, 2005; Chantraine \& Sallée, 2013; Sallée, 2013), les CEF restent encore peu étudiés, notamment en Suisse romande, en dehors de certains travaux historiques (Ruchat, I993; Heller, 2012). Si l'enquête en milieu carcéral s'avère particulièrement délicate en raison des contraintes inhérentes aux institutions fermées, le fait même que des sociologues puissent étudier ce type d'univers était peut-être l'indice d'un «contexte de desserrement [relatif] de l'étau carcéral» (Bouagga, 2013: 22). Financée par la Confédération suisse (CTI) ${ }^{7}$ et répondant à un mandat de recherche du Département de la sécurité du canton concerné, 8 l'enquête a montré combien l'analyse des conditions d'enquête gagnait à être intégrée dans le processus de construction de l'objet étudié. La présentation des conditions de production d'une recherche apparaît le plus souvent aux yeux des chercheur.e.s comme un préalable à l'analyse. Or, cette exigence de réflexivité peut aussi être envisagée comme «un véritable matériau d'analyse du terrain lui-même» et «non pas seulement de la position de l'enquêteur, de ses réussites ou de ses échecs, ou de sa plus ou moins grande capacité, à négocier son entrée sur le terrain" (Darmon, 2005: 99). Dans certains cas, les conditions d'enquête participent à la définition même de l'objet de recherche. Une telle perspective redéfinit inévitablement le moment où débute l'enquête et élargit le corpus des matériaux empiriques analysé. Nous allons voir que c'est à travers l'analyse du contexte dans lequel la demande institutionnelle s'inscrivait que nous avons pu transformer une sollicitation d'expertise en réalisation d'une véritable démarche de recherche sociologique en milieu carcéral pour mineur.e.s. Il s'agissait alors, en quelque sorte, de porter une attention particulière aux «conflits

6 «L'ensemble est délimité par des murs, quelquefois des grillages, jamais de barbelés ou de barreaux; il existe rarement de caméras de surveillancemais toujours des portails automatiques pour l'accès. Ces deux éléments, comme parfois d'autres, viennent symboliser la clôture davantage que des murs ou des barrières, l'idée étant d'en faire un lieu de vie plutôt qu'un espace d'enfermement» (Lenzi \& Milburn, 20I5).

7 La Commission pour la technologie et l'innovation (CTI), rebatisée Innosuisse depuis 20I8, est l'agence pour la promotion de l'innovation; elle fait partie du Département fédéral de l'économie, de la formation et de la recherche.

8 Pour préserver l'anonymat du terrain d'enquête et des personnes interviewées, nous avons invisibilisé le canton concerné. 
et contradictions » traversant la réalité étudiée, à l' "épaisseur » du présent, ou encore «à dégager derrière la demande » des «configurations problématiques" (Castel, 2004: 72ss).

«Savez-vous où vous mettez les pieds?»

Si nos démarches d'enquête ont été relativement bien reçues au niveau de l'administration pénitentiaire et de la direction du CEF étudié, nous avons pourtant dû, tout au long de notre enquête, négocier, voire renégocier notre place. Non pas que nous subissions des restrictions particulières, mais la situation de crise pesait sur l'état d'esprit des professionnel.le.s et la perspective de voir à nouveau des regards experts venir les ausculter ne les réjouissait guère. Les professionnel-le.s étaient donc réticent.e.s à devoir se prêter à de nouvelles investigations, partageant parfois le sentiment d'un: «Pourquoi ça tombe sur nous? » (Beaud, \& Weber, 2003: I I 8). L'expression souvent énoncée par certains membres de la direction à notre intention était révélatrice à cet égard: "Savez-vous où vous mettez les pieds? " Cet avertissement de la direction conjugué aux réticences des équipes de terrain étaient aisément compréhensibles. En effet, entre 2010 et 20I I, en plus de l'invalidation du concept d'intervention proposé par la direction du "Verger» par l'instance fédérale chargée de son financement, le CEF avait fait l'objet de trois rapports commandités par diverses instances publiques, vécus chaque fois par les professionnel-le.s de terrain comme autant d'audits. En outre, depuis une dizaine d'années, l'établissement faisait régulièrement l'objet d'articles de presse reflétant des critiques de la prison bien connues (Foucault, I975: 309-3I2): "Surpopulation carcérale», «mauvaises conditions de détention ", "non-application des nouveaux standards internationaux", situations de violence assimilées parfois à des «mini-émeutes», "évasions" répétées de certains jeunes détenu·e•s, telle était la teneur des titres des articles ${ }^{9}$ publiés dans la presse romande au sujet du CEF. La critique énoncée était souvent contradictoire: l'institution était incriminée en raison du caractère trop répressif ou inhumain de certaines pratiques - non-respect de certains droits des mineur·e•s, arrivée de «matons » dans l'institution, ou encore nombre trop élevé de personnes par chambre -, mais aussi en raison de son incapacité à pouvoir maintenir certaines prérogatives qui lui seraient pourtant constitutives, comme la capacité à enfermer les jeunes détenu·e•s en évitant les évasions. Dans ce contexte chargé, autant dire que la situation d'enquête n'était guère évidente. Dès lors, il convenait 
de rassurer les personnes enquêtées quant à nos intentions. Par souci de souligner la spécificité de notre démarche, descriptive et analytique, nous prenions grand soin de rappeler chaque fois cette caractéristique aux informatrices et informateurs rencontrés. Il ne s'agissait pas pour nous d'évaluer si les professionnel.le.s en faisaient «assez» ou "pas assez», si leur intervention était «répressive» ou «trop laxiste», si les nouveaux standards internationaux en matière de droits des mineur.e.s étaient suffisamment respectés ou pas, ou encore si la régulation des populations détenues était encore «lacunaire» ou les mailles du filet suffisamment fines ou efficaces, ni si la collaboration entre groupes professionnels était adéquate ou non.

Nous souhaitions étudier ce que les personnes enquêtées faisaient, ou avaient fait, et ce, de leur point de vue. Mais nous souhaitions également prendre la mesure des interactions dans lesquelles s'inscrit leur activité professionnelle et prendre en compte ce que faisaient les autres autour, avec, contre, voire sur elles. Il s'agissait alors d'aller à la rencontre des différents acteurs et actrices professionnels, de leurs expériences et de leur point de vue. Cette démarche suppose que les individus soient, bien sûr, en mesure de donner un peu de leur temps tout en se trouvant dans des «conditions sociales [favorables] à la prise de parole» (Beaud, I996: 234). Paradoxalement ou pas, les conditions de travail difficiles auxquelles étaient exposés les professionnel.le·s dans le CEF étudié ${ }^{10}$ (Gaspar \& Tran, 20I6) les ont plutôt incités à prendre la parole et à faire valoir leur point de vue durant les entretiens. Certaines personnes interviewées voyaient peut-être dans notre démarche de compréhension sociologique une occasion de rendre compte des difficultés particulières auxquelles elles étaient confrontées?

Une institution déjà pensée par des savoirs d'expertise

Cela étant dit, une partie des réticences des actrices et acteurs institutionnels envers nos démarches d'enquête tenait sans doute à notre identité disciplinaire, à notre casquette de sociologue, posture soucieuse de conjuguer

9 Une trentaine d'articles de presse relatifs au «Verger» ont été publiés entre I998 et 2012 .

Ces conditions de travail s'étaient péjorées dans le CEF étudié depuis la dernière décennie. Ce dernier faisait l'objet d'un taux d'absentéisme élevé ainsi que de nombreuses formes d'épuisement professionnel. 
compréhension et distanciation critique plutôt qu'expertise et conseil en matière de bonnes pratiques. Mobilisant un point de vue sociologique, nos démarches n'ont pas toujours été évidentes, ou bien comprises, tant cette lecture ne va pas de soi dans cet univers social particulier et court souvent le risque d'apparaître comme «extérieure", "surplombante», sans être pour autant totalement inconnue de certaines personnes enquêtées. En effet, notre présence dans ce CEF a semblé réveiller parfois certains épisodes de l'histoire récente, associés à la réception difficile des lectures critiques de Michel Foucault (I975) ou d'Erving Goffman (I968) portant sur les institutions d'enfermement.

Question: Je ne sais pas si je peux poser une question, mais est-ce qu'il y a alors un lien entre la psychopathologie et le fait de se retrouver en prison? Réponse: Il y a une étude nationale qui a été commanditée par l'Office fédéral de la justice qui montre bien qu'on a $70 \%$ à $75 \%$ de jeunes qui ont des troubles psychiques dans l'ensemble des centres éducatifs de la Suisse. [...] Tous les chiffres montrent qu'on a autant de psychopathologies dans ce type d'institution que dans les institutions carcérales en général. Mais c'est peutêtre l'idée qu'au contact des psychiatres, on devient plus malade? C'est peutêtre pour ça aussi, qu'on paraît aussi, menaçant [...] Mais aussi je pense qu'il y a autre chose... Je pense, je pense déjà que c'est Foucault qui voyait chez les psychiatres des gens qui étaient des captureurs d'âmes, mais aussi des gens qui travaillaient finalement de manière normative. Les psychiatres, du coup, se sont toujours défiés aussi de vouloir être trop dans cette représentation-là. (Grégoire, membre de la direction médicale)

Révélateurs des effets qu'ont pu produire sur la psychiatrie et ses institutions certains savoirs critiques, les propos du médecin psychiatre n'informent-ils pas, également, sur la dynamique de transformation de la psychiatrie publique, soucieuse de sortir d'une délimitation pathologique de sa mission pour s'engager vers une santé mentale plus proche des gens, moins stigmatisante (Coutant, 20I2) ? Une chose est sûre: «Les réactions des membres d'un groupe donné à l'existence du sociologue ne peuvent pas ne pas livrer des indices sur leur image d'eux-mêmes, sur les types de légitimité qu'ils revendiquent, sur les formes de reconnaissance auxquelles ils aspirent, donc sur les «noyaux durs » ou les aspects fragiles de leur identité sociale» (Schwartz, I993: 276). L'embarras de certaines personnes enquêtées, ici du médecin psychiatre face au sociologue, était, en creux, révélateur de la constitution historique des savoirs légitimes autour 
de cet objet. Cette situation renseignait sur une situation de concurrence des savoirs, ici entre les sociologues venant de l'extérieur et les expert.e.s œuvrant à l'intérieur des quatre murs du «Verger» (Castel, I99I). En nous indiquant parfois comment penser le CEF tout en invalidant certaines grilles de lecture, il est certain qu'une certaine définition sociale de «ce qu'enfermer des jeunes veut dire" était engagée par les personnes enquêtées. Cette situation ne pouvait que nous inciter, dès lors, à prendre pour objet d'étude la production sociale de cette économie morale de l'enfermement dans la diversité de ses luttes symboliques.

Des difficultés d'enquête nous mettant sur une piste de recherche féconde

Cette piste de recherche a également été confirmée par des changements survenus en cours d'enquête. Alors que nous venions de réaliser trois entretiens exploratoires, une volonté de diminuer les temps d'encellulement - en phase avec les attentes de l'Office fédéral de la justice, mais aussi de certaines recommandations européennes ${ }^{11}$ - a été relayée par les autorités locales de la juridiction des mineur.e.s au moyen d'une directive à destination des professionnel.le.s, les enjoignant à «sortir les jeunes des cellules au minimum huit heures par jour». Entrant en contradiction avec certains discours publics, repris par les médias locaux, demandant à sécuriser davantage l'institution afin d'éviter les évasions et de maintenir l'ordre interne, la diminution des temps d'encellulement renseignait aussi sur la pression toujours plus forte des cadres réglementaires extérieurs sur les professionnel.le.s du «Verger». La référence grandissante à de nouveaux standards juridiques et aux droits des personnes détenues nous informait, sous un autre angle, que c'est bien la question du sens conféré à l'enfermement qui faisait débat. Les modalités de notre entrée sur le terrain et les difficultés qui l'ont accompagnée nous ont donc rapidement amené·e·s à considérer la question du sens de l'enfermement "en actes " comme l'énigme sociologique à laquelle il s'agissait de répondre. Confronté.e.s à

Recommandations qui postulent à l'article 80.I: «Le régime doit permettre aux mineurs de passer autant d'heures que possible hors de leur chambre pour disposer d'un degré d'interaction sociale approprié. Ils devraient pouvoir bénéficier d'au moins huit heures par jours à cette fin" (Recommandation CM/Rec (2008) I I du Comité des Ministres sur les Règles européennes pour les délinquants mineurs faisant l'objet de sanctions ou de mesures). 
un CEF traversé par une réforme pénale en train de se faire, nous avons pris le parti d'appréhender l'institution à partir de «ceux qui concrètement, au quotidien, la font» (Coutant, 201 2: 24), à savoir les professionnel.le.s qui travaillent entre ses murs. Nous sommes donc allé.e.s à la rencontre des maîtresses et maîtres socioprofessionnels, du corps éducatif (groupes professionnels déjà présents dans le CEF depuis sa création, au milieu des années I960), mais aussi des agent.e.s de détention et du personnel médical et enseignant, nouveaux entrants arrivés dans l'institution durant les années 2000.12 Notre travail de compréhension sociologique a consisté, non pas à décréter le sens de l'enfermement des mineur.e.s aujourd'hui en CEF, mais à rendre compte plus modestement de ces "points de vue» situés dans l'espace local (Bourdieu, I993), et cela dans la diversité de leurs manifestations, dans les tâtonnements, oscillations et résistances des agent·e•s qui mettent concrètement en œuvre l'action publique.

\section{La réception de nos analyses sociologiques}

A travers la réalisation de notre enquête, il nous est apparu progressivement que trois processus organisaient la fabrique du sens de l'enfermement en CEF :

, une division technique et morale du travail comme creuset d'une recomposition des territoires d'intervention;

, des conceptions éducatives plurielles investies d'une légitimité inégale selon leur distance aux normes dominantes;

, une institution totale qui demeure... par-delà l'ouverture sur la réalité extérieure.

Ces thèses ont fait l'objet d'une réception contrastée et sélective de la part de nos enquêté.e.s: ce sont notamment les rivalités de territoire d'intervention et la pertinence du concept goffmanien d'«institution totale» (I968) qui ont suscité le plus de réactions auprès des personnes enquêtées. Avant de documenter leurs attitudes, rappelons à grands traits la portée de nos analyses.

Concernant les transformations du champ d'intervention, nos résultats ont montré que, au "Verger», l'arrivée de nouveaux corps professionnels (agent.e.s de détention en 2002, professionnel.le.s de la santé en 2005 et de l'enseignement en 2008) va produire un déplacement de l'axiomatique dominante du champ local d'intervention professionnelle reconfigurant en 
profondeur les territoires d'intervention. Alors que les nouveaux entrants sont amenés à découvrir de nouveaux espaces d'intervention, les groupes déjà installés - éducateurs et éducatrices, MSP - se voient contraints de recomposer le périmètre de leur action face à l'arrivée des nouveaux venus. $\mathrm{Si}$, auparavant, "chacun.e faisait un peu de tout", l'analyse montre combien les controverses professionnelles induites par les processus de division du travail et de différenciation des rôles portent, certes, sur des aspects techniques reposant sur la maîtrise différente de savoirs et savoir-faire nécessaires à l'accomplissement de tâches spécialisées, mais aussi et peutêtre surtout sur des aspects moraux renvoyant à l'inégale désirabilité de la répartition des tâches (Hughes, I997). A bien des égards, les attitudes contrastées des groupes professionnels déjà installés face aux transformations de leur territoire d'intervention, les controverses sur les limites du rôle de chacun•e, les résistances exprimées sur la nature du travail délégué, ou encore la déroute de voir son périmètre d'action - comme celui des MSP - se rétrécir sans que ce réaménagement s'accompagne d'une revalorisation témoignent de la recomposition de la division du travail engagée depuis les années 2000. En effet, ce champ d'intervention professionnel ne saurait se réduire à une simple juxtaposition de territoires professionnels complémentaires, comme le suggèrent parfois certains discours institutionnels tenus en matière d'organisation du travail en réseau ou de gestion d'un environnement professionnel pluridisciplinaire. De fait, cette nouvelle répartition des rôles induite par l'arrivée de nouveaux venus se manifeste sous une forme à la fois saillante symboliquement, mais aussi parfois chargée d'affects. De fait, c'est notamment sous l'angle des luttes de concurrence entre professionnel.le.s de l'écoute et de formes de résistance au «sale boulot» que l'enquête a documenté la nouvelle division morale du travail et la recomposition des territoires d'intervention.

Concernant la pertinence du concept d'«institution totale», nos analyses se sont efforcées de revisiter cette lecture goffmanienne en mettant en avant tant les ruptures que les continuités. Construite par Erving

Notre enquête au sein du «Verger» repose sur la réalisation de vingt-deux entretiens qualitatifs individuels: cinq maîtresses et maîtres socio-professionnels (MSP), neuf éducateur.e.s, deux agent.e.s de détention, un médecin, un infirmier et une enseignante, trois membres de la direction. Ce dispositif méthodologique s'est accompagné de quatre entretiens collectifs (focus groups), ainsi que de l'analyse d'un corpus documentaire. 
Goffman à partir des hôpitaux psychiatriques, mais étendue à cinq groupes d'institutions (prisons, hôpitaux, casernes, foyers pour indigents, monastères...), l'institution totale est un concept idéaltypique utilisé pour désigner «un lieu de résidence et de travail, où un grand nombre d'individus, placés dans la même situation, coupés du monde extérieur pour une période relativement longue, mènent ensemble une vie recluse dont les modalités sont explicitement et minutieusement réglées " (Goffman, I968: 4I). Ce concept insiste donc sur le caractère contraignant de l'institution, les barrières qu'elle dresse contre les échanges avec l'extérieur, les techniques de mortification qu'elle met en place pour marquer la rupture avec la vie d'avant et la coupure entre la vie recluse et la vie normale. ${ }^{13}$ Dans tous les cas, le succès public du livre, Asiles. Etudes sur la condition sociale des malades mentaux, sera lié à son inscription dans les critiques institutionnelles et le mouvement anti-psychiatrique des années I970. Concernant les changements observables, nous avons amplement documenté la dynamique profonde de décloisonnement expérimentée au "Verger», qui s'inscrit dans un processus plus général de «détotalisation" (Rostaing, 2009) de l'institution-prison, censée répondre à la critique de la «désocialisation » induite par l'enfermement. Nous avons montré comment un processus de détotalisation des lieux d'enfermement tendait alors à ébranler la dichotomie "dans les murs»/ «hors les murs», au cœur du projet goffmanien d'appréhension des institutions totales, au profit d'une attention nouvelle à l'inscription sociale et spatiale de la prison dans son environnement, notamment urbain, et à leurs influences réciproques (Combessie, I993; I996; Sallée, 20I2). Si nos analyses restituent cette dynamique d'ouverture, elles ont également soulevé une hypothèse forte de recomposition de l'institution totale par-delà les murs. Cette dynamique était observable, notamment via certaines mises à l'épreuve des jeunes détenu·e.s sur l'extérieur, expérimentées à une certaine phase de la mesure d'observation. Plus qu'une ouverture réelle des jeunes sur l'extérieur, ces autorisations de sortie révèlent la force des contraintes qui pèsent sur ces jeunes détenu·ess et les exigences normatives auxquelles ils doivent se plier pour pouvoir sortir - notamment, l'adhésion à l'injonction très forte de «responsabilité». Derrière la multiplicité des regards professionnels observant les jeunes, ces analyses témoignent également d'une extension du contrôle au-delà des murs de l'institution. ${ }^{14}$ En ce sens, l'ouverture, relative, de l'institution aux proches des jeunes détenu·e•s en conférant, par exemple, à leurs parents un rôle à jouer dans la construction d'un projet 
de sortie, peut avoir pour effet d'augmenter la contrainte pesant sur leurs épaules - en termes de responsabilité et de contrôle de la trajectoire tout autant que concernant la possibilité de réinsertion des jeunes détenu.e.s sans forcément les dégager véritablement d'une forme de «stigmate carcéral", comme le montre l'analyse de Touraut (20I2). Enfin, nos analyses ont suggéré combien, derrière la valorisation de la dimension «contenante» de l'institution, un aspect très souvent valorisé par les professionnel.le.s rencontrés, ${ }^{15}$ on retrouvait au fond le caractère enveloppant des institutions totales, une des caractéristiques identifiées par Goffman. ${ }^{16}$

Des attitudes contrastées selon les groupes professionnels...

La restitution de nos analyses relatives à la division sociale du travail d'encadrement au "Verger» a suscité des réactions très contrastées et parfois virulentes de la part des professionnel-le·s. Ces réactions demeuraient à première vue étranges, au regard des garde-fous méthodologiques utilisés. En effet, cherchant à étudier conjointement différents groupes professionnels et afin d'éviter de se voir assigner à un groupe et de se trouver alors

La traduction française de «total» par «totalitaire» insiste sur la première partie de l'ouvrage, alors que la deuxième partie souligne davantage les possibilités de résistance des personnes recluses; ouvrage complexe, susceptible d'alimenter des lectures plurielles.

Le constat de ces continuités entre l'intérieur et l'extérieur des murs, théorisées par Wacquant (200I) sous le terme de "continuum carcéral", tend à "décarcéraliser» l'approche de l'enfermement par les sciences sociales (Darley, Lancelevée \& Michalon, 2013). A cet égard, on assiste à un processus ambivalent, à la fois de «décarcéralisation» et de renforcement/extension de la contrainte carcérale, qui trouve dans le bracelet électronique sans doute son point le plus achevé. Cette alternative à la détention - permettant de surveiller les déplacements de condamné.e-s hors détention - aurait pour corollaire une transformation profonde de la dimension spatiale de l'enfermement (Razac, 2009; Devresse, 20I2). On retrouve également derrière la notion d' «aide contrainte», parfois mobilisée par les enquêté.e.s, une notion qui connaît, en matière de protection des mineur.e-s, un certain écho auprès des professionnel-le·s depuis la publicisation, notamment, des travaux du thérapeute systémicien Guy Hardy (20I2).

Vienne $(2005: 67)$ rappelle combien la notion d'espace «enveloppant» s'inscrit pour Goffman dans une logique de totalisation, où l'auteur «vise donc ici moins l'absence ou la présence de caractère résidentiel dans l'institution, que la capacité de celle-ci à réaliser une emprise sur ses membres, à les insérer dans un univers spécifique». 
dans l'impossibilité d'enquêter sur les autres, le défi du dispositif d'enquête mobilisé consistait de manière générale à «montr[er] un égal intérêt pour tous les groupes» (Avril et al., 2010: 89). A cet égard, notre ancrage institutionnel à la Haute école de travail social de Genève a joué tant en notre faveur qu'en notre défaveur. Il a, certes, a priori facilité nos démarches auprès de certains corps professionnels, comme les éducatrices et éducateurs, corps professionnel en partie formé dans ce type de structure de formation; en même temps, cet ancrage risquait aussi de compromettre notre souci d'enquêter conjointement, avec la même profondeur analytique, sur les autres groupes professionnels. Conscients de ces risques, nous avons pris le soin «de rendre public et visible notre intérêt pour tous les groupes, de l'extérioriser (et de le verbaliser explicitement) plutôt que de chercher la position invisible et neutre» (Avril et al., 20ı0: 89-90).

Deux restitutions, à des moments différents de l'enquête, ont été faites au sein même du «Verger» et en présence de l'ensemble de son personnel. Une première restitution des résultats provisoires de l'enquête, organisée à mi-chemin sur la base d'une présentation du travail au «Verger », a permis de publiciser cette préoccupation et a été, d'une manière générale, très bien accueillie. De nombreuses poignées de mains furent effectuées à l'issue de cette présentation. A y regarder de plus près, certains groupes professionnels - comme les MSP, notamment - qui demeuraient partiellement déroutés par les changements occasionnés par la transformation du champ d'intervention professionnel, semblaient témoigner d'une forme de reconnaissance du travail de recherche accompli toute particulière. Cette réaction était sociologiquement compréhensible. Nous montrions en effet que l'esprit d' "ouverture » symbolisé par la nécessité de l'accès des jeunes détenu.e.s à un régime de droit commun en détention - dynamique présente dans d'autres contextes nationaux - se soldait notamment par la mise en concurrence entre groupes professionnels autour du type d'activité et d'occupation à favoriser pour tourner ces jeunes vers l'extérieur, concurrence où les MSP étaient mis à mal. Ainsi en allait-il de la place réduite des ateliers manuels qui, malgré leur capacité apparente à recréer un environnement de travail proche du monde extérieur, sont concurrencés par les autres activités pédagogiques et thérapeutiques proposées, faisant suite au rétrécissement des temps d'encellulement et au souci d' «activer» les jeunes (Solini \& Basson, 20I2). Lors de la présentation finale de l'enquête à l'ensemble des corps professionnels qui y travaillent, exception faite du corps médical, des réactions et des dynamiques similaires ont été autant 
observées que lors de la restitution intermédiaire. Cela tend à confirmer le sentiment de manque de reconnaissance qu'éprouvent les MSP et le corps enseignant au sein du «Verger» dans la division sociale du travail et donc, en creux, la reconnaissance de leur position que notre analyse leur témoignait. Les dynamiques des prises de parole - publiques et affirmées du côté des éducatrices et éducateurs et de la direction, en privé à la fin de la présentation pour les MSP, la lingère et l'enseignant - ont également rejoué à leur façon les rapports de force entre professionnel.le.s.

Une troisième restitution a été faite lors d'une journée d'étude organisée en décembre 2013 à la HETS de Genève ${ }^{17}$ à l'issue du rapport de recherche. Contrairement aux deux autres moments de restitution, celui-ci n'avait pas explicitement pour objectif de rendre compte de nos résultats aux corps professionnels du «Verger», mais de présenter notre travail tant à un public composé de professionnel.le.s issus de différents champs du travail social qu'à des chercheur.e.s. Nous avions invité l'ensemble des corps professionnels du «Verger», mais ce sont principalement des membres du corps éducatif qui ont assisté à notre présentation dans le cadre de cette journée d'étude. Celle-ci a suscité des réactions vives et parfois contrariées de la part de professionnel.le.s du «Verger» présent.e.s. Certaines personnes représentant le groupe des éducateurs estimaient ainsi que la spécificité de leur travail d'encadrement des jeunes n'avait pas été suffisamment mise au centre de la conférence publique. Toute une partie des matériaux empiriques présentés ce jour-là documentait, il est vrai, davantage le travail du personnel médical. Il convenait de prendre aux sérieux ces réactions, de même que l'effet de lieu associé au cadre de notre présentation. La journée de recherche était en effet organisée à la HETS, une institution qui forme des éducatrices et éducateurs pouvant être amenés à travailler au «Verger». Par ailleurs, se trouvaient, dans le public, d'autres professionnel.le.s du travail social dont certains pouvaient être relativement critiques à l'égard du travail social en milieu fermé. Dans ce sens, on peut alors faire l'hypothèse que les éducatrices et éducateurs sont les professionnel.le.s du «Verger» ressentant le plus grand clivage entre les

17 Une journée d'étude à laquelle participaient également l'historienne vaudoise Geneviève Heller, ayant réalisé une belle étude sur la maison d'éducation de Vennes (20I2), et le sociologue Gilles Chantraine, chargé de recherche au CNRS et spécialiste de la sociologie de la prison, ayant enquêté notamment dans les établissements pénitenciers pour mineur·e·s en France (EPM). 
termes antinomiques de "centre éducatif» et "fermé», et qui sont donc le plus mis en danger par nos résultats soulignant les affinités croissantes du corps éducatif avec les notions de «contenance» ou d'«éducation sous contrainte». En témoignent ces propos tenus en privé par une éducatrice au terme de la restitution: "Je me suis dit qu'après la lecture de ce rapport, personne ne m'emploiera ailleurs ", qui illustrent bien une peur d'être perçu par un employeur lambda comme exerçant une fonction éducative répressive.

Par ailleurs, au-delà de la critique d'une certaine invisibilité du personnel éducatif dans la présentation de nos résultats, les réserves énoncées publiquement par certains éducateurs et éducatrices envers notre présentation renvoyaient, en creux, à certaines controverses éprouvées à l'interne avec le personnel médical, que nous retrouvions également dans ce contexte «hors les murs». Là aussi, nos analyses trouvaient une forme de validation ex post. L'arrivée du corps médical en 2005 au «Verger» engage un déplacement de l'axiomatique dominante, caractérisée par le déplacement d'une conception «réaliste» du lien avec l'extérieur - insertion dans le monde du travail -, au profit d'une conception pédagogico-thérapeutique orientée autour de la fabrique d'un sujet «responsable», capable d'identifier ses sources de risques, ses ressources et les situations pouvant conduire à un comportement délinquant (Quirion, 20I2). L'importance commune conférée par le personnel éducatif et médical au fait d'«être dans la relation", une conception de l'intervention reposant sur une forme de "gouvernement par la parole» (Bugnon, 20I4), s'accompagnera de petites rivalités en la matière, qui se manifestent dans la critique croisée d'instrumentalisation de la relation par la médication que les éducatrices et éducateurs adressent au corps médical ou de réduction de la relation à la définition du cadre contraignant et sanctionnant que le corps médical adresse au corps éducatif. Ces rivalités entre corps professionnels s'accompagnent d'un certain brouillage du vocabulaire en usage entre corps médical et éducatif. Les référentiels cognitifs du soin ont été aisément appropriés et mobilisés par le personnel éducatif, groupe professionnel extérieur au monde médical stricto sensu. Emancipée du langage strictement médical, l'idée de soin reste toutefois marquée de manière sous-jacente par son empreinte. Si bien que les éducatrices et les éducateurs semblent occuper une position symboliquement moins avantageuse que les membres du personnel médical dans ces luttes symboliques orientées autour du monopole de définition légitime de la «bonne» prise en charge, ajustée aux besoins des 
détenu.e.s. En même temps, le personnel éducatif représente le corps professionnel qui demeure au "Verger», sur le plan quantitatif, le plus représenté parmi l'ensemble des professionnel.le.s œuvrant dans l'institution et pouvant s'appuyer, en outre, sur un certain capital d'ancienneté (Elias \& Scotson, I997 [1965]).

Concernant notre tentative de revisiter le concept d'institution totale goffmanien en en soulignant tant les ruptures que les continuités, notre présentation publique à la HETS semble avoir réveillé les ambiguïtés du concept d'institution totale. Certain.e.s membres de la direction du «Verger» semblaient fortement dérangé.e.s par cette expression en soi: «Le Verger n'est pas une institution totalitaire, vous n'avez pas compris? ", tant celle-ci leur semblait désormais désuète, sinon inappropriée pour caractériser l'institution étudiée et rendre compte de sa dynamique réformatrice, notamment de décloisonnement vis-à-vis de l'extérieur. Cette réaction renvoie à ce que nous avions expérimenté avec le corps médical et à la méfiance à l'égard de la lecture critique foucaldienne ou goffmanienne des institutions contenantes. Elle met ainsi en lumière une forme de récupération institutionnelle de la critique de l'institution totale (Rostaing, 2009). Elle souligne également la volonté de ces actrices et acteurs de se distancer de la logique sécuritaire du CEF pour mettre l'accent sur sa dimension humaniste. In fine, elle révèle les tensions et contradictions qui traversent les institutions fermées (Bouagga, 2013).

Finalement, cette analyse sur la réception des résultats de recherche nous amène à considérer l'importance du contexte de restitution: dans l'entre-soi des murs du "Verger», les professionnel-le.s étaient prêt.e.s à admettre la complexité de leurs relations de travail ainsi que l'inertie de certaines logiques historiques mais, lors d'une présentation publique qui mettait en scène leurs pratiques professionnelles quotidiennes face à des enseignant·e.s, des chercheur.e.s et des collègues, il s'agissait avant tout de sauver la face de l'institution.

... révélatrices de la pertinence de nos hypothèses de recherche

Ainsi, les attitudes recueillies à l'occasion des moments de restitution se sont révélées riches d'enseignements. Les modes de lecture sélectifs et les réinterprétations parfois arrangeantes de notre recherche se présentaient alors comme un test ultime de la pertinence de nos hypothèses, selon laquelle la signification conférée à l'enfermement des mineur·e•s 
faisait l'objet de luttes symboliques au sein du champ d'intervention étudié; luttes de reconnaissance et de concurrence entre différents groupes professionnels expliquant, pour une large part, l'orientation nouvelle, cognitive et morale, conférée à «ce qu'enfermer des jeunes veut dire». Ainsi, les regrets exprimés tantôt par certains corps professionnels, tantôt par d'autres, à l'occasion de certaines de nos présentations orales, de voir leurs pratiques moins «visibilisées » que celles d'autres groupes, venaient enrichir encore notre analyse ${ }^{18}$ : l'émergence d'une nouvelle axiomatique du champ d'intervention en CEF, qui se manifeste par une certaine appréhension du «bon» travail en milieu fermé, où prédomine le souci d'être «dans la relation» avec les jeunes, et des vertus - faire réfléchir, responsabiliser, rendre disponible à la relation - que le cadre «contenant» peut induire auprès de jeunes en termes de modifications d'attitude, du rapport à soi et aux autres. Cette sorte d'appréhension collective de «ce qu'il convient de faire", à laquelle chaque actrice et acteur professionnel était tenu implicitement de se conformer, présente cette propriété particulière de réunir tout en divisant chacun des groupes professionnels autour de valeurs implicites conférées aux pratiques d'encadrement, mais dont les moyens à disposition pour les faire reconnaître demeurent inégalement distribués, différences pouvant jouer parfois au sein d'un même groupe professionnel (Milly, 2004). Enfin, la dynamique réformatrice visant à «détotaliser» le CEF étudié que nous documentions ne signifiait guère la nécessité, sur le plan scientifique, de remettre en cause fondamentalement la pertinence du cadre d'analyse goffmanien, ${ }^{19}$ même s'il est indéniable qu'un examen critique s'imposait (Amourous \& Blanc, 200I ; Rostaing, 200I). L'ensemble de ces situations de restitution contribuait en un sens à la validation de notre objet d'étude, centré sur la production sociale de l'économie morale de l'enfermement dans la diversité de ses luttes symboliques, luttes enfermant aussi des enjeux politiques de représentation de l'institution vis-à-vis de l'extérieur.

La réception de cette recherche menée dans un CEF romand demeure, selon les quelques occasions de restitution ou de publicisation que nous avons pu expérimenter jusque-là, variable selon les contextes et les groupes professionnels concernés. A l'occasion de la négociation d'une nouvelle recherche, portant cette fois sur l'expérience des jeunes face à la justice pénale, ${ }^{20}$ nous sommes retournés au «Verger» en janvier 2017. Un membre de la direction exprima alors une attitude équivoque à notre propos: "On vous connaît, mais je ne suis guère rancunier. "Sans doute accédons- 
nous progressivement au "statut d'étranger sympathique" (Olivier de Sardan, I995) pour certain.e.s professionnel.le.s du «Verger»? Ce statut nous permettant, cette fois, d'aller à la rencontre des jeunes détenu.e.s $\mathrm{au}$ «Verger» et de découvrir ainsi «par le bas » un autre visage de la justice pénale et de ses effets, recherchés ou non, (in)désirables.

\section{Conclusion}

On a vu combien l'analyse des conditions de production d'une recherche sociologique dans un CEF de Suisse romande avait contribué à mettre l'équipe de recherche sur une piste de recherche féconde (constituer le sens de l'enfermement des mineur·e.s en objet d'étude sociologique), alors que celle des modalités de réception des produits de l'enquête pouvait représenter l'occasion d'une mise à l'épreuve des hypothèses dégagées. Si l'analyse des «coulisses» de la production et de la réception de la recherche au «Verger» est heuristique sur le plan de la production des connaissances scientifiques, on soulignera enfin que le terrain d'enquête expose les chercheur.e.s à un «terrain difficile», aussi en raison du rapport entretenu par les sciences sociales envers le champ pénal, une relation qui varie, de fait, fortement selon les contextes nationaux. Allié critique et constructif en Allemagne, où le rôle "social» de la recherche en sciences sociales dans ce domaine est revendiqué, ce que traduit le concept de Begleitforschung, la recherche comme «accompagnement»; en France, prévaut davantage l'idée de distanciation par rapport aux autorités publiques, comme garantie

Les mêmes personnes pouvaient exprimer des attitudes et des points de vue différenciés quant à notre travail selon le contexte d'énonciation. Ces variations nous poussaient alors à réfléchir sur les contraintes différentes (publiques et officielles versus "privées» et personnelles) pesant sur la prise de parole et les censures induites tacitement par certains contextes d'énonciation.

Cadre caractérisé par une dimension idéaltypique, de par la diversité des structures institutionnelles potentiellement analysables dans cette perspective: foyers fermés, hôpitaux, prisons, couvents, mais aussi casernes, navires en mer ou internats.

20 Et dont l'expérience du milieu fermé ne demeure qu'une dimension possible parmi d'autres. Cf. Frauenfelder, Schultheis, Bugnon \& Weil, Les jeunes face à la justice: analyse de la chaîne pénale à travers les expériences et trajectoires des justiciables. Projet FNS (Division I), 30 mois (2016-2019). 
nécessaire d'un travail pleinement indépendant et critique (Salle, 2003). En Suisse romande, les sciences sociales ont - mis à part certains travaux historiques ${ }^{21}$ - encore très peu investi ce genre d'univers, raison pour laquelle enquêter en CEF constitue une démarche scientifique à la fois passionnante et semée d'embûches.

21 A l'image de la genèse du dernier PNR 76: «Assistance et coercition - passé, présent et avenir ", où la perspective historique a joué un rôle central dans l'élaboration scientifique de ce programme.

\section{Références bibliographiques}

Amourous, C. \& Blanc, A. (Dir.) (200I). Erving Goffman et les institutions totales. Paris: L'Harmattan.

Avril, C., Cartier, M. \& Serre, D. (20I0). Enquêter sur le travail. Concepts, méthodes, récits. Paris: La Découverte.

Beaud, S. (1996). L'usage de l'entretien en sciences sociales. Plaidoyer pour l'«entretien ethnographique», Politix, 9(35), 226-257.

Beaud, S. \& Weber, F. (2003). Guide de l'enquête de terrain. Paris: La Découverte.

Becker, H. S. (2002). Les ficelles du métier. Comment conduire sa recherche en sciences sociales. Paris: La Découverte.

Boumaza, M. \& Campana, A. (2007). Enquêter en milieu «difficile». Revue française de science politique, 57 (I), 5-25.

Bourdieu, P. (I993). «Comprendre». Dans P. Bourdieu (Dir.), La misère du monde (pp.903-939). Paris: Seuil.

Bourdieu, P. \& Wacquant, L. (20I4). Invitation à la sociologie réflexive. Paris: Seuil.

Bugnon, G. (20I4). Gouverner par la liberté. Normalisation des subjectivités et contrôle contractuel dans la mesure de Liberté Assistée au Brésil. [Thèse de doctorat en sociologie]. Genève/Lille: Université de Genève et Université de Lille I.

Castel, R. (I99I). Savoirs d'expertise et production de normes. Dans F. Chazel \& J. Commaille (Dir.), Normes juridiques et régulation sociale (pp. I77-I88). Paris: LGDJ.

Castel, R. (2004). La sociologie et la réponse à la demande sociale. Dans B. Lahire (Dir.), A quoi sert la sociologie? (pp.67-77). Paris: La Découverte.

Chantraine, G. \& Sallée, N. (20I3). Eduquer et punir. Travail éducatif, sécurité et discipline en établissement pénitentiaire pour mineurs. Reuue française de sociologie, 54(3), 437-464.

Coutant, I. (20I2). Troubles en psychiatrie. Enquête dans une unité pour adolescents. Paris: La Dispute.

Darley, M., Lancelevée, C. \& Michalon, B. (2013). Où sont les murs? Penser l'enfermement en sciences sociales. Cultures \& Conflits, 9o. Consulté le 24.I0.2013 sur http:/conflits.revues. org/18703 
Darmon, M. (2005). Le psychiatre, la sociologue et la boulangère: analyse d'un refus de terrain. Genèses, I (58), 98-I I 2.

Devresse, M.-S. (20I2). Investissement actif de la sanction et extension de la responsabilité. Le cas des peines s'exerçant en milieu ouvert. Déviance et société, $36(3), 3$ I I-323.

Elias, N. \& Scotson, J. L. (I997) [I965]. Logiques de l'exclusion. Enquête sociologique au cœur des problèmes de communauté. Paris: Fayard.

Fassin, D., Bouagga, Y., Coutant, I., Eideliman, J.-S., Fernandez, F., Fischer, N., Kobelinsky, C., Makaremi, C., Mazouz, S. \& Roux, S. (2013). Juger, réprimer, accompagner: essai sur la morale de l'Etat. Paris: Seuil.

Foucault, M. (I975). Surveiller et punir. Naissance de la prison. Paris: Gallimard.

Frauenfelder, A., Bugnon, G. \& Nada, E. (20I5). "S'ouvrir sur l'extérieur»: une réforme saisie par les professionnels d'un centre éducatif fermé. Espaces et sociétés, I62, I I I-I 26.

Frauenfelder, A., Nada, E. \& Bugnon, G. (20I4). "Savez-vous où vous mettez les pieds? » Enquêter dans un centre éducatif fermé pour mineurs. Cultures et sociétés, 30, 68-74.

Frauenfelder, A., Nada, E. \& Bugnon, G. (20I5). Division morale du travail et recompositions du sens de l'enfermement en Centre éducatif fermé. Déviance et société, 39(4), 477-500.

Frauenfelder, A., Nada, E. \& Bugnon, G. (2018). Ce qu'enfermer des jeunes veut dire. Enquête dans un centre éducatif fermé. Genève/Zurich: Seismo.

Gaspar, J.-F. (2012). Tenir! Les raisons d'être des travailleurs sociaux. Paris: La Découverte. Gaspar, J.-F. \& Tran, S. (20I6). Dans les murs. Travail social en maison de peine: effet de lieu, effet d'usure. SociologieS, Dossiers. Récupéré le 21.06.20I 8 de https://journals.openedition.org/sociologies/5365

Goffman, E. (1968). Asiles. Etudes sur la condition sociale des malades mentaux. Paris: Minuit.

Hardy, G. (20I2). S'il te plaît, ne m'aide pas! L'aide sous injonction administrative et judiciaire. Toulouse: Erès.

Heller, G. (20I2). Ceci n'est pas une prison. La Maison d'éducation de Vennes. Histoire d'une institution pour garçons délinquants en Suisse romande (I805-I846-I 987). Lausanne: Antipodes.

Hughes, E. C. (I997) [I97I]. Le regard sociologique. Essais choisis. [Textes rassemblés et présentés par J.-M Chapoulie]. Paris: EHESS.

Lenzi, C. \& Milburn, P. (20I5). Les centres éducatifs fermés: de la clôture institutionnelle à l'espace éducatif. Espaces et sociétés, I62(3), 95-I Io.

Milly, B. (2004). L'enseignement en prison: du poids des contraintes pénitentiaires à l'éclatement des logiques professionnelles. Déviance et société, 28(I), 57-79.

Mucchielli, L. (2005). Les centres éducatifs fermés: rupture ou continuité dans le traitement des mineurs délinquants. Revue d'histoire de l'enfance «irrégulière» (RHEI), 7, I I3-I46.

Office fédéral de la justice (2013), "Coup de projecteur: foyers d'éducation fermés », Bulletin info/2, Berne, OFJ.

Olivier de Sardan, J.-P. (I995). La politique du terrain: sur la production des données en anthropologie. Enquête, I, 7 I-Iog. 
Quirion, B. (2012). Réformer, réadapter ou responsabiliser le détenu. Analyse des enjeux normatifs rattachés à l'intervention correctionnelle au Canada. Déviance et société, 36(3), 339-355.

Razac, O. (2009). Histoire politique du barbelé. Paris: L'Harmattan.

Rostaing, C. (200I). Pertinence et actualité du concept d'institutions totales: à propos des prisons. Dans C. Amourous \& A. Blanc (Dir.), Erving Goffman et les institutions totales (pp. I37-I53). Paris: L'Harmattan.

Rostaing, C. (2009). Interroger les changements de la prison. Des processus de déprise et de reprise institutionnelle. Tracés. Revue de sciences humaines, I7, 89-108.

Ruchat, M. (1993). L'oiseau et le cachot. Naissance de l'éducation correctionnelle en Suisse romande I800-I9I3. Genève: Zoé.

Salle, G. (2003). Situation(s) carcérale(s) en Allemagne. Prison et politique. Déviance et société, 27(4), 389-4I I.

Sallée, N. (20I4). Les mineurs délinquants sous éducation contrainte. Responsabilisation, discipline et retour de l'utopie républicaine dans la justice française des mineurs. Déviance et société, 38(I), 77-102.

Schwartz, O. (1993). L'empirisme irréductible. Dans A. Nels, Le hobo. Sociologie du sans-abri [Postface]. Paris: Nathan.

Solini, L. \& Basson, J.-C. (20I2). L'hyperactivité forcée: un mode de gestion des mineurs incarcérés. Dans R. Bodin (Dir.), Les métamorphoses du contrôle social (pp. I67-I77). Paris: La Dispute.

Touraut, C. (20I 2). La famille à l'épreuve de la prison. Paris: Presses Universitaires de France.

Vienne, Ph. (2005), De l'institution totale à l'institution scolaire. La grille de lecture goffmanienne d'une ethnographie scolaire dans l'enseignement professionnel. La matière et l'esprit, 2, 63-8I.

Wacquant, L. (200I), Symbiose fatale. Quand ghetto et prison se ressemblent et s'assemblent. Actes de la recherche en sciences sociales, 3(I39), 3I-52. 


\title{
Posture du ou de la chercheur.e dans la relation d'entretien avec des jeunes ayant des parcours migratoires difficiles
}

\author{
Théogène-Octave Gakuba
}

\section{Introduction}

Mener des recherches sur des jeunes ayant des parcours migratoires difficiles peut mettre les chercheur·e.s dans une posture délicate, surtout s'ils sont perçus par les personnes enquêtées comme faisant partie de leur communauté, c'est-à-dire s'ils partagent certaines caractéristiques communes, notamment le lieu d'origine, la langue, la religion, des éléments d'histoire de vie. Pour certain.e•s jeunes migrant.e•s, l'arrivée dans le pays d'accueil peut être vécue comme une situation traumatisante quand ils se retrouvent, par exemple, sans famille ou confronté.e.s aux difficultés d'intégration (problème de statut de séjour, non-maîtrise de la langue, difficultés scolaires, discrimination). Parfois, ces jeunes ont subi des situations de guerre et de violence dans leur pays d'origine avant le départ en exil. Leurs histoires de vie sont des sujets sensibles qui relèvent de la vie privée et qui influencent le déroulement de l'entretien et les postures des enquêtrices ou enquêteurs.

Pour aborder ma posture de chercheur, je me réfère, dans cet article, à deux recherches ${ }^{1}$ que j'ai menées avec des jeunes réfugié.e.s et migrant.e.s. Les entretiens semi-directifs conduits portaient sur des thèmes en rapport avec leur histoire personnelle parfois émouvante, leur vécu psychologique et leurs parcours migratoires difficiles. Dans le cas de la recherche portant sur les jeunes Rwandais·e·s réfugié·e.s en France et en Suisse, j'ai, par exemple, eu affaire à des jeunes qui avaient vécu des événements

1 Une recherche menée par Théogène-Octave Gakuba, Ibrahima Guissé et Gislène Zürcher, en collaboration avec Tatiana Oyomo Amougou. Pour davantage de détails, voir Gakuba (2013, 2004). 
traumatiques durant la guerre, le génocide et l'exil. Dans la mesure où l'entretien de recherche est considéré comme une interaction entre deux partenaires ou plus (Olivier de Sardan, I995), sa dimension relationnelle concerne non seulement les échanges établis entre les personnes impliquées dans l'entretien, mais aussi les sentiments éprouvés à l'égard d'autrui dans cette interaction. Dans le cas de cette recherche doctorale, j'ai enquêté sur des compatriotes rwandais. Même si la raison de mon arrivée en Suisse est due aux études et a eu lieu avant le génocide des Tutsi en avril I994 et les massacres des Hutus au Rwanda, mon engagement personnel dans cette recherche est évident, du fait que, en choisissant de travailler sur la résilience des jeunes Rwandais·e.s réfugié.e.s, je voulais comprendre les conséquences psychologiques des événements vécus par mes compatriotes et les ressources mobilisées pour les surmonter, dans le but d'apporter ma contribution à leur prise en charge psychosociale.

Les entretiens de recherche avec les jeunes migrant.e.s qui ont connu des histoires de vie difficiles ont soulevé un certain nombre de questions, que je souhaite discuter à partir des cas qui seront présentés dans cet article: comment gagner la confiance des jeunes qui ont vécu des événements traumatiques afin d'obtenir leur participation active dans un entretien de recherche? Comment réagir aux histoires émouvantes ou à la souffrance des jeunes au cours des entretiens? Quelle posture adopter face aux jeunes migrant.e.s qui expriment des demandes à propos de leurs projets personnels? Quels sont les avantages ou les inconvénients d'être une chercheure ou un chercheur perçu par les enquêté.e.s comme faisant partie de leur communauté?

L'article se divise en quatre parties: la première présentera de manière succincte les recherches et la deuxième discutera des questions évoquées ci-dessus en mettant l'accent sur la relation que j'ai entretenue avec les jeunes migrant.e.s et réfugié.e.s dans les entretiens. Ma posture dans des situations problématiques d'entretien sera ensuite abordée. Enfin, je montrerai la pertinence de la restitution des résultats de mes recherches. 
9I | Posture du ou de la chercheur.e dans la relation d'entretien...

\section{Présentation des recherches}

Recherche sur le processus d'intégration des jeunes issus de l'immigration africaine subsaharienne en Suisse romande (Genève, Vaud)

En Suisse, l'immigration africaine est récente, contrairement aux immigrations européennes traditionnelles, caractérisées par l'arrivée de travailleuses et travailleurs issus des régions frontalières d'Allemagne, de France, d'Autriche, d'Italie, avant et après la Seconde Guerre mondiale (Piguet, 2004). La présence africaine en Suisse devient conséquente entre les années I970 et I990, avec l'arrivée de demandeuses et demandeurs d'asile angolais, érythréens, éthiopiens, zaïrois (ressortissant·e•s de l'actuelle République démocratique du Congo) et somaliens. C'est la période où les ressortissant.e.s de certains pays africains fuyaient les guerres civiles (Angola et Somalie), les régimes dictatoriaux (ex-Zaïre) et la famine (Ethiopie et Erythrée).

Les données de l'Office fédéral de la statistique ${ }^{2}$ montrent que presque toutes les nationalités africaines sont représentées en Suisse, y compris celles des pays anglophones et lusophones. Actuellement, les demandeuses et demandeurs d'asile érythréens représentent un grand nombre des Africain.e.s qui viennent en Suisse. La majorité des ressortissant.e.s d'Afrique subsaharienne réside principalement dans les cantons de Genève, Berne, Vaud et Zurich.

Comparativement à d'autres communautés étrangères, les migrant.e.s d'Afrique subsaharienne présentent des caractéristiques particulières, notamment le vécu traumatique de certaines personnes ayant quitté leur pays d'origine au moment d'une guerre civile et se retrouvant dans une situation de précarité sociale (Eyer \& Schweizer, 20I0). Les migrant.e-s originaires d'Afrique subsaharienne vivant en Suisse connaissent également un accès difficile à l'emploi (Bagalwa \& Bolzman, 20I4). La situation précaire de la plupart des familles africaines immigrées ainsi que les difficultés d'ordre socio-culturel (langue, changement de statut social, adaptation à certaines valeurs en Suisse) sont des facteurs pouvant générer des

2 Site de l'OFS consulté le 23.04.20I8, https://www.bfs.admin.ch/bfs/fr/home/statistiques/population/migration-integration.html 
conflits au sein des couples. Cette situation entraîne parfois des divorces ayant des répercussions sur le bien-être psychologique des enfants et sur leurs projets d'avenir. Par exemple, dans les familles érythréennes et somaliennes en Suisse, l'augmentation des divorces est notamment due à la redéfinition des rôles des hommes et des femmes (Eyer \& Schweizer, 2010).

Dans cette étude, la question générale était de savoir comment se fait le processus d'intégration des jeunes issus de l'immigration africaine subsaharienne en Suisse. Je suis parti de l'hypothèse suivante: il existe un lien entre les situations pré-migratoires, migratoires et post-migratoires complexes des familles immigrantes d'Afrique subsaharienne et les difficultés d'intégration (chômage, difficultés d'accès aux formations professionnelles et supérieures, insuffisance de réseaux sociaux, non-participation à la vie politique et sociale) que rencontrent un certain nombre de jeunes.

La recherche, originale puisqu'il y avait peu d'études sur les jeunes migrant.e.s africain.e.s en Suisse, avait les objectifs suivants:

- Fournir un ensemble d'informations utiles à une meilleure compréhension du processus d'intégration en Suisse des jeunes migrant.e.s d'Afrique subsaharienne (I8 à 25 ans), à travers l'expérience de deux groupes cibles:

> celui de la «deuxième génération» (les personnes nées en Suisse)

, celui des personnes nées à l'étranger (pays d'origine ou autre pays)

et arrivées en Suisse après la scolarité obligatoire

, Donner aux professionnel.le.s du social et de la santé et aux responsables politiques des outils qui leur permettraient de comprendre les problèmes liés à l'intégration socioprofessionnelle de ces jeunes, mais aussi mettre en évidence les ressources dont ces jeunes disposent.

, Identifier des pistes d'intervention psychosociale auprès des jeunes migrant·e·s ayant vécu des situations de guerre et de violence dans leur pays d'origine.

Recherche sur la résilience des jeunes Rwandais·e.s réfugié·e·s en France et en Suisse

Cette recherche avait pour objectif d'étudier le concept de résilience (Cyrulnik, I998), qui était d'actualité au début des années 2000, en l'appliquant à la situation concrète de jeunes Rwandais·e.s réfugié.e.s en France et en Suisse, ayant fui la guerre et le génocide. Il s'agissait d'analyser les 
conséquences psychologiques de la guerre et de l'exil chez ces jeunes, mais aussi leur capacité de les surmonter. L'utilisation de manière complémentaire de trois méthodes (les entretiens individuels semi-directifs, le questionnaire semi-structuré pour le stress post-traumatique du DSM-IV, l'échelle de l'estime de soi de Rosenberg, I986) m'a permis d'atteindre cet objectif. En me concentrant sur le concept de résilience, j'ai suivi des jeunes durant quatre ans en discutant régulièrement avec eux de leur situation. Cette démarche longitudinale a été choisie dans la logique d'envisager la résilience comme «une caractéristique non stable pour la personne mais plutôt une capacité qui se construit dans un processus continu, durant toute une vie» (Vanistandael \& Lecomte, 2000: I85).

L'analyse du contenu des entretiens semi-directifs avec les jeunes et du questionnaire semi-structuré pour le stress post-traumatique a révélé leur souffrance psychologique liée à la guerre (menace d'être tué·e, voir une personne être tuée ou blessée) et à la situation d'exil (quitter le pays d'origine, attente du statut de réfugié.e, problème d'adaptation au nouvel environnement culturel, peur d'être expulsé•e). Les plus vulnérables sont des orphelin.e.s qui n'ont pas pu ritualiser leur deuil et les jeunes séparé.e.s de leurs parents en raison d'un placement dû aux actes délinquants qu'ils ont commis. La souffrance psychologique se manifeste aussi chez les jeunes par un sentiment de métamorphose identitaire (Gakuba, 2004), c'est-àdire le fait d'avoir changé sur le plan cognitif, affectif et social à cause des événements vécus pendant la guerre. Un certain nombre de jeunes Rwandais.e.s sont cependant parvenus à surmonter leur souffrance psychologique grâce aux ressources personnelles et sociales dont ils disposaient. Les principales ressources qui ont joué un rôle déterminant dans leur processus de résilience sont notamment les valeurs culturelles de la société d'origine (notion de responsabilité, intelligence sociale, volonté de maîtrise de soi, références religieuses), l'école, le groupe d'ami.e•s fidèles sur le long terme, la présence d'une personne de confiance et de l'entourage familial, qui peuvent faire modèle d'identification, et le sentiment d'appartenance à sa communauté d'origine.

\section{Aspects méthodologiques et éthiques des recherches}

Comme on l'a vu plus haut, la démarche de recherche adoptée dans ces deux études s'inscrit dans un paradigme compréhensif et explicatif utilisant des méthodes qualitatives. La démarche par triangulation, consistant à 
combiner plusieurs méthodes de collecte de données (Cohen \& Manion, I980, cités par Pourtois \& Desmet, I988), a permis de multiplier les sources d'information et de procéder à leurs recoupements.

Terrain d'enquête et population

Le terrain de l'étude sur le processus d'intégration des jeunes issus de l'immigration d'Afrique subsaharienne était les cantons de Genève et Vaud. Ces cantons comptent un grand nombre de migrant.e.s d'Afrique subsaharienne, ce qui a permis de mobiliser facilement les jeunes pour l'enquête. L'échantillon était composé de vingt-six jeunes (neuf filles et dix-sept garçons), âgé.e.s de 18 à 25 ans. Les jeunes qui ont accepté de participer à l'enquête ont des parents venant de divers pays d'Afrique subsaharienne (Angola, Bénin, Burkina-Faso, Burundi, Erythrée, Ethiopie, Cameroun, CapVert, Congo, Maurice, Nigeria, Rwanda, Somalie, République démocratique du Congo) et sont issus de familles présentant des situations différentes. Sept jeunes ont des parents qui vivent ensemble, alors que seize ont des parents divorcés. Deux jeunes ont perdu leurs deux parents, un jeune a des parents restés en Afrique. Au moment de l'entretien, vingt-quatre jeunes étaient en formation tandis que les deux autres avaient terminé leurs études et étaient à la recherche d'un emploi.

L'échantillon de recherche comprenait également des professionnel.le.s du social et de la santé ainsi que des responsables d'associations impliqué.e.s dans l'insertion socioprofessionnelle des jeunes dans les cantons de Genève et Vaud.

S'agissant de l'étude sur la résilience des jeunes Rwandais·e·s, elle a été menée dans les villes de Bordeaux, Lyon, Orléans, Paris et Tours en France, et dans les cantons de Berne, Genève, Lausanne, Fribourg et Neuchâtel en Suisse. Ces villes ont été chosies du fait qu'il s'y trouvait une grande communauté rwandaise. L'échantillon de l'étude était composé de trente jeunes Rwandais·e.s (seize jeunes en France et quatorze en Suisse, dont quatorze garçons et seize filles) arrivé·e·s en France et en Suisse entre I994 et 200 I. Quinze jeunes étaient demandeurs et demandeuses d'asile (onze en Suisse et quatre en France), onze avaient un statut de réfugié.e (en France), une personne avait une carte de séjour pour étudiant.e en France, deux jeunes avaient un permis B humanitaire en Suisse, un jeune avait un permis de séjour B en Suisse. Concernant leur situation familiale, dix jeunes étaient orphelin·e.s, dont sept orphelin.e.s de père et de mère, alors que douze jeunes ne vivaient pas avec leurs parents, soit parce qu'ils étaient réfugiés 
dans d'autres pays africains ou européens, soit parce qu'ils sont restés au Rwanda. La majorité des vingt-six personnes interviewées étaient en formation et quatre avaient un travail sans qualification.

Pour les deux échantillons, l'insertion professionnelle des parents dans le pays d'accueil était difficile. Bien que les parents aient effectué des études secondaires ou universitaires dans leur pays d'origine, la majorité occupaient des emplois non qualifiés, d'autres bénéficiaient de l'aide sociale.

Entretiens individuels semi-directifs

Les thèmes abordés dans les entretiens individuels semi-directifs avec les jeunes ont porté sur:

, La formation, l'insertion professionnelle, les conditions de vie et la participation sociale;

, L'identité culturelle (transmission des valeurs culturelles de la société d'origine, perception de la société suisse ou française par rapport à la société d'origine);

, Les représentations des jeunes par rapport à l'avenir;

, Les problèmes psychologiques présentés par les jeunes en exil;

, Les ressources personnelles et sociales contribuant à la résilience.

Questionnaire semi-structuré pour

le stress post-traumatique du DSM-IV

Le questionnaire semi-structuré pour le stress post-traumatique (SIPTSD) du DSM-IV permet de donner des éléments de réponse à l'une des questions de recherche sur les symptômes du stress post-traumatique et les problèmes psychologiques présentés par les jeunes. Cette interview comprend des questions qui s'articulent autour de cinq critères d'évaluation (cf. American Psychiatric Association, DSM-IV, I996: 503-504):

, Vécu du traumatisme;

, Revivre l'événement traumatique;

, Evitement persistant des stimuli associés au traumatisme (efforts pour éviter les pensées, les activités, incapacité de se rappeler d'un aspect important du traumatisme, réduction nette de l'intérêt pour des activités importantes, sentiment de détachement d'autrui, restriction des affects, sentiment d'avenir bouché);

, Présence de symptômes persistants traduisant une activation neurovegétative (difficultés d'endormissement ou sommeil interrompu, 
irritabilité ou accès de colère, difficulté de concentration, hypervigilance, réaction de sursaut exagérée);

, Sentiment de culpabilité des personnes survivantes.

A chaque critère, il importe de spécifier si la durée des symptômes est de moins de trois mois (forme aiguë) ou si la durée des symptômes est de trois mois ou plus (chronique).

J'ai retenu les symptômes qui ont duré trois mois ou plus. L'interview structurée pour le stress post-traumatique a permis de compléter les informations données dans les entretiens semi-directifs sur les problèmes psychologiques. La durée de l'interview structurée pour le stress posttraumatique a été en moyenne d'une heure et demie.

Dans les deux recherches, je me suis respectivement référé au code d'éthique de la Haute école de travail social de Genève (HETS, 2009). ${ }^{3}$ Avant de commencer l'entretien avec les jeunes et les professionnel.le·s, je les ai d'abord informé.e.s des buts de la recherche, des méthodes de recueil des données, des conditions de l'entretien (enregistrement, droit de réserve si on ne souhaite pas répondre à une question, confidentialité du contenu d'entretien, anonymisation des personnes interviewées). Ensuite, j'ai sollicité leur consentement pour participer à la recherche. Leur accord a été donné de manière orale, dans la mesure où je n'ai pas jugé indispensable de leur faire signer un document écrit pour ne pas casser la confiance établie au moment du recrutement. Presque toutes les personnes sollicitées ont donné leur accord oral de participation. Elles ont également accepté d'être enregistrées, à l'exception de trois personnes. J'ai respecté leur choix. Ces enjeux éthiques m'ont amené à m’interroger sur ma façon de gagner la confiance des jeunes ayant vécu des parcours migratoires difficiles.

\section{La confiance des jeunes avec des parcours migratoires difficiles s'établit lors du recrutement}

Gagner la confiance des jeunes qui devaient participer à mes études était une condition primordiale au déroulement des enquêtes, et plus particulièrement à la réussite des entretiens (Poupart, Deslauriers, Groulx, Laperrière, Mayer \& Pires, I997). La plupart des jeunes Rwandais·e.s avaient en effet vécu des événements traumatiques dans leur pays d'origine et durant leurs parcours migratoires. Ces événements avaient créé chez eux un sentiment 
de peur, de méfiance et de manque d'estime de soi. D'autres jeunes, qui étaient passés par le processus de l'asile, avaient, par exemple, été marqués par les auditions sur les motifs de l'asile menées par les fonctionnaires du Secrétariat d'Etat aux Migrations (SEM). C'est le cas de ce jeune, qui a mal vécu sa première audition:

«La première interview que j'ai faite ici en Suisse m'a énervé même plus que tout ce que j'avais vu au Rwanda, celui qui m'a interrogé m'a dit que j'étais comme les autres Africains qui viennent en Suisse pour chercher de l'argent, il m'a dit que je mentais pour demander l'asile. C'est là où j'ai senti le point culminant du traumatisme» (Isidore, interviewé en 2000). ${ }^{4}$

Gagner la confiance des jeunes a ainsi commencé par leur recrutement en les convaincant de participer à la recherche. Le recrutement des jeunes réfugié·e·s rwandais·e.s a exigé de faire connaissance avec eux ainsi qu'avec des parents et des membres de la communauté rwandaise exilés en France et en Suisse.

Dans les deux échantillons, pour recruter les jeunes, j'ai utilisé la technique de l'échantillonnage «boule de neige», c'est-à-dire que je suis passé par les jeunes que je connaissais et qui m'ont aidé à atteindre d'autres jeunes. Cette technique a bien fonctionné, dans la mesure où les personnes qui étaient recrutées par leurs ami.e·s étaient rassurées et se sentaient en confiance pour participer à la recherche. Je me suis également entretenu avec certains parents, qui ont essayé de convaincre leurs enfants. L'échantillon constitué à partir de la technique «boule de neige était hétérogène, du point de vue de l'histoire personnelle de chaque jeune et du point de vue de la situation familiale et du niveau socio-économique des parents.

Dans l'étude sur le processus d'intégration des jeunes issus de l'immigration africaine subsaharienne en Suisse romande, je me suis informé sur les lieux où je pouvais recruter ces jeunes et sur leurs conditions de vie, notamment de celles et ceux ayant passé par le processus de l'asile. J'ai ainsi pris du temps pour créer des contacts avec des intermédiaires, notamment les associations de migrant.e.s africain.e.s et les structures d'accompagnement juvénile, qui m’ont permis d'établir les contacts recherchés.

3 Voir https://www.hesge.ch/hets/sites/.../travail_social/codeethiquets-tb-etat_ avril2oi3.pdf

4 Les prénoms utilisés dans les citations sont fictifs pour préserver l'anonymat des jeunes. 
Mon réseau social au sein de la communauté africaine installée dans les cantons de Genève et de Vaud m'a aussi été très utile.

Le recrutement des jeunes a, par ailleurs, été rendu possible grâce à la collaboration avec les responsables d'institutions sociales et d'associations qui travaillent dans le domaine de la migration dans les cantons de Genève et Vaud. Le statut de chercheur de la Haute école de travail social a facilité mes contacts avec les responsables de ces structures et associations qui, pour la plupart, sont des partenaires privilégiés de la HETS. Les responsables contacté.e.s ont montré leur intérêt pour mon étude, d'autant plus qu'ils comprenaient l'utilité des résultats dans l'accompagnement psychosocial de ce public. Pour recruter les jeunes, les responsables leur expliquaient d'abord l'objet de mon étude, avant de leur demander leur accord pour que je les contacte en vue d'un entretien de recherche. Je téléphonais alors à celles ou ceux qui avaient laissé leur numéro pour les informer des buts de la recherche et de mes attentes, avant de solliciter un rendez-vous dans le cas d'une réponse favorable.

Les personnes les plus enthousisates à participer à la recherche me considéraient comme faisant partie de leur communauté, du fait de mon origine africaine, de mon histoire migratoire et de ma connaissance des langues africaines parlées par certain.e.s jeunes (kinyarwanda, kirundi, swahili). Ce sentiment d'appartenance à la même communauté a ainsi créé un climat de confiance avec les jeunes. La familiarité entretenue avec ces personnes m'a donné l'impression d'être considéré comme un alter ego, un semblable et un aîné (Haas \& Masson, 2006), qui pouvait leur donner des conseils en fonction de ses expériences en Suisse. D'un autre côté, en tant que chercheur universitaire, j'étais dans une relation asymétrique avec ces jeunes qui, pour certain.e.s, étaient encore en formation ou en recherche d'emploi, mais je leur ai témoigné mon respect. J'ai essayé d'établir une relation plutôt symétrique avec les jeunes, en leur expliquant qu'ils avaient des choses à m'apprendre et ne devaient pas avoir peur de s'exprimer librement. Sanchez-Mazas, Maggi et Roca I Escoda estiment à ce sujet «qu'un entretien qui s'inspire d'une approche ethnographique visant la proximité et l'établissement d'un cadre empathique accordant une égale validité à la perspective du chercheur et à celle de l'acteur dans une relation symétrique devrait favoriser l'émergence de la voix d'acteurs affaiblis » (2010: I48).

Le fait d'être considéré comme un alter ego et un aîné m'a cependant mis dans une posture embarrassante quand certain.e.s jeunes ont imaginé que je pouvais les aider à résoudre leurs problèmes de la vie quotidienne, 
notamment concernant l'obtention d'un emploi ou d'un stage d'apprentissage. Chaque fois que j'étais sollicité, j'expliquais aux jeunes mes limites par rapport à leurs attentes, mais j'essayais de les orienter en leur fournissant des informations sur des structures existantes à Genève et les personnes qui pourraient les accompagner.

\section{La posture de la ou du chercheur.e face à la souffrance des jeunes dans les entretiens}

Comme je l'ai déjà souligné, j'ai abordé, dans mes deux recherches, des sujets sensibles puisque les jeunes interviewés étaient invités à s'exprimer sur leurs parcours migratoires difficiles, notamment sur les événements vécus pendant la guerre et le génocide au Rwanda. L'intimité des jeunes était explorée à double titre (entretien semi-directif et questionnaire de type médical sur le stress post-traumatique) d'autant plus que la posture de chercheur.e implique de garder une certaine distance, afin de permettre à la personne d'exprimer ses réponses de la façon la plus «indépendante» possible. Je devais ainsi m'adapter aux caractéristiques propres de mes interlocuteurs et interlocutrices durant les entretiens (Boutin, I997).

Au cours des entretiens, je me trouvais, dans la plupart des cas, devant des personnes démunies, présentant des problèmes affectifs et émotionnels, qui me parlaient de leur souffrance psychologique ou de leur sentiment d'injustice et de révolte contre leur situation migratoire. Les portraits et extraits d'entretien qui suivent montrent la posture que j'ai adoptée face à la souffrance psychologique de trois jeunes.

Fatou: le rituel de deuil non accompli

Fatou a fui le Rwanda à l'âge de sept ans, en I994. Elle est arrivée dans un pays européen en I998 avec sa mère adoptive et ses autres sœurs pour demander l'asile, qui leur a été accordé deux ans plus tard. Fatou avait I4 ans lors de l'entretien. Dans son enfance, elle a connu des difficultés liées à la pauvreté, à la maladie incurable de sa mère qui, finalement, n'a pas survécu alors que Fatou était encore au Rwanda. Le jour de l'enterrement de sa mère, Fatou n'a pas assisté aux funérailles à cause des problèmes de déplacement dus à la guerre dans son pays. Pendant le génocide, Fatou a failli être tuée et a dû se cacher dans le plafond de la maison 
pendant plusieurs jours. Quelques mois après son arrivée en France, les événements traumatiques vécus pendant la guerre, les traumatismes liés au rituel de deuil non accompli ont commencé à resurgir avec intensité chez Fatou, qui évoque sa souffrance psychologique dans les entretiens en ces termes:

«C'est maintenant que tous les événements que j'ai vécus dans la guerre et même avant viennent me traumatiser, c'est pourquoi je vais voir le psychologue. Tout me vient dans ma tête, tout ce que j'ai vécu que j'ai fait semblant de ne pas voir, c'est maintenant que tout ça se réveille, c'est-à-dire que maintenant je paie le prix. Le psychologue m'a dit que je suis traumatisée.»

La reviviscence des événements traumatiques chez Fatou est associée à d'autres problèmes psychologiques et symptômes de stress post-traumatique. Elle a plusieurs symptômes: blocage de pensées, solitude, silence, mensonges, sentiment de culpabilité, idées suicidaires.

"Je n'arrive pas à parler de mes problèmes sauf quand je fais semblant. C'est un masque que les gens voient, ce n'est pas moi qu'ils voient. En fait, quand je rigole, c'est pour leur faire plaisir, mais dans ma tête ça bouillonne. Une fois, j'étais en classe, je ne sais pas ce qui s'est passé, j'ai tremblé et les autres me demandaient si j'étais malade...»

La répression de la souffrance et des sentiments chez Fatou s'accompagne de gestes d'automutilation et d'évacuation de l'agressivité:

"Je me coupe avec des ciseaux et ça, je le fais souvent, j'ai des cicatrices parce que quand je souffre, ça diminue la douleur. Avant de dormir, je fais la boxe contre les murs.» Elle est par ailleurs menacée dans sa santé par le fait qu'elle fume.

Le récit de la souffrance de Fatou ne me laissait pas insensible et je ne me sentais pas à l'aise, du fait que mon statut de chercheur ne me permettait pas d'apporter une aide psychologique à la jeune fille pour alléger sa souffrance. Je ne pouvais que faire preuve d'écoute et d'empathie en essayant de comprendre son histoire et en lui racontant mon vécu d'orphelin de père durant mon adolescence pour essayer de me mettre à sa place. J'estimais que cette posture me rendait plus empathique dans le sens de Rogers (I980, cité par Decety, 2004: 59), qui indique que «être empathique, c'est percevoir le cadre de référence interne d'autrui aussi précisément que possible et avec les composants émotionnels et les significations qui 
lui appartiennent comme si l'on était cette personne, mais sans jamais perdre de vue la condition du "comme si».

Cette posture de compréhension et d'intervention dans l'entretien en parlant de mon histoire personnelle a permis à Fatou de se sentir comprise à propos de sa souffrance et d'avoir les ressources pour continuer à parler de son vécu. Donner son avis dans l'entretien est en effet considéré par Legavre comme un moyen d'aider l'enquêté·e à «sortir de lui-même»... et à faire qu'il en dise plus que ce qu'il aurait probablement dit dans une autre situation d'entretien "classique» (I996: 22I).

Jean: la perte de la mère durant son enfance

Jean a perdu sa mère pendant le génocide au Rwanda dans des circonstances dramatiques, quand il avait 8 ans. Avec son père, ils sont arrivés en Suisse pour demander l'asile. Au moment de l'entretien, Jean avait I6 ans. Durant l'entretien semi-structuré pour le stress post-traumatique, j'ai abordé les événements traumatiques et posé la question de la perte de sa mère. Jean ne s'est pas exprimé sur ce sujet et j'ai appris ensuite que son père avait décidé de ne pas lui en parler avant qu'il ne soit majeur. J'avais donc abordé un sujet tabou, ce qui a posé des problèmes à l'interviewé qui ne souhaitait pas répondre à d'autres questions. Après l'entretien, Jean s'est enfermé dans sa chambre à la maison, a pleuré toute la soirée, n'a voulu parler à personne ni manger. Son père, qui ne comprenait pas le comportement de son fils, m'a appelé chez moi très tard le soir pour me demander ce que j'avais fait à son enfant. Je lui ai expliqué comment l'entretien s'était déroulé et les thèmes abordés.

En discutant avec le père, j'ai compris que le fait d'avoir parlé de la mort de la mère avait affecté l'adolescent. Celui-ci a pu surmonter cette crise grâce à l'écoute de son père. Je me suis senti mal à l'aise, avec un sentiment de culpabilité d'avoir provoqué une situation douloureuse chez l'adolescent dont le père m'avait fait confiance en m'autorisant à interviewer son fils. Je me suis excusé auprès du parent et du jeune mais, avec le recul, j'estime que je n'aurais pas dû faire l'entretien avec cet adolescent, qui n'était pas encore prêt à parler de sa souffrance psychologique. Je n'avais pas suffisamment discuté avec le père pour analyser les risques de l'entretien. Dans l'entretien, il convient en effet de tenir compte «des caractéristiques sociales des catégories de populations interrogées (les sujets de l'enquête) mais aussi des rapports qu'elles entretiennent avec les questions et thèmes abordés (les objets de l'enquête)" (Demazière, 201 2: 36) 
D'autres jeunes ont aussi montré leurs émotions, leur souffrance et leur angoisse quand ils ont évoqué leurs parcours migratoires, leur situation difficile en exil, leur désespoir face à l'avenir incertain. En tant qu'être humain, il m'était impossible de rester insensible à la détresse de ces jeunes, même si je devais garder une certaine distance en tant que chercheur. J'ai expliqué à ces jeunes que mon objectif n'était pas de les prendre psychologiquement en charge, mais je leur donnais quelques conseils pour faire face à leurs problèmes. Je leur montrais l'importance qu'il y aurait à consulter des psychologues, à discuter avec leurs ami.e.s proches ou les membres de leur famille ou de leur communauté d'origine. Il convient également de souligner que d'autres jeunes ont manifesté leur satisfaction d'être interviewés, dans la mesure où ils n'avaient jamais exprimé leurs traumatismes auprès de psychologues. Ces jeunes avaient besoin d'être écoutés dans un entretien considéré, dans le sens de Sanchez-Mazas et Roca i Escoda (2007), comme une figure de réconfort ou comme une mise à disposition attentive de soi qui offre l'ouverture d'un capital de reconnaissance.

Pierre: le sentiment de ne pas être reconnu comme suisse

Pierre, jeune Suisse d'origine africaine, avait 2I ans au moment de l'entretien. Il est né en Suisse de parents africains et possède la double nationalité (suisse et celle du pays d'origine de ses parents). En s'exprimant, sur le thème de l'identité, Pierre a mis en évidence le sentiment de ne pas être reconnu comme suisse en ces termes:

"C'est clair que je suis fier de mon pays d'origine, même plus fier, car en Suisse, j'y suis né, je me suis habitué et c'est pourquoi je m'y plais au minimum. Mais je ne pourrais jamais brandir le drapeau suisse, fier d'en être patriote, je pense que c'est impossible pour moi. La Suisse, partout où je vais, quand je dis que je suis suisse, les gens rigolent, même mes amis, même si ce n'est pas méchant, ça les fait rire. Ça veut tout dire, c'est la situation. Ici, il y a une mentalité car il est encore inconcevable qu'un Suisse ne soit pas de couleur blanche. Déjà, un Italien ou un Espagnol naturalisé suisse, ça va encore et les Suisses ne vont pas tout de suite trop se poser la question. Mais pour moi, non! ‘D’origine, ah d'accord, tu es né ici et puis tes parents sont venus à la nage? ? Ils vont tout de suite se poser la question. Tous ces facteurs font en sorte que je ne puisse pas me sentir chez moi, tout simplement.»

Dans ce témoignage, le jeune parle de la discrimination des personnes naturalisées suisses qui ne sont pas blanches. Une situation qui témoigne 
de la racialisation dont il est l'objet et provoque sa colère ainsi que son manque de patriotisme envers la Suisse. Parmi les jeunes interviewés, trois ont évoqué le fait de ne pas être reconnu comme suisses. Mon statut de chercheur d'origine africaine a sans doute influencé leurs propos. Certain.e.s jeunes ont en effet profité de l'entretien pour parler de leurs déceptions et frustrations, ce qui apparaissait comme tout à fait légitime. De surcroît, une occasion leur a été donnée durant les séances de restitution des résultats; ils ont pu parler de leur vécu en présence d'autres jeunes qui n'avaient pas participé à l'enquête et de professionnel.le.s du social et de la santé investi.e.s dans la migration.

\section{Restituer les résultats de la recherche pour partager la souffrance des jeunes migrant.e.s}

Après la fin des recherches précitées, j'ai gardé des liens avec certain·e·s jeunes qui m'avaient pris comme une personne ressource; c'est-à-dire que je pouvais les aider dans leurs difficultés de la vie quotidienne. Je leur ai aussi envoyé un exemplaire de mon étude. Nous avons continué à échanger sur leurs conditions de vie et sur leurs projets. J'ai également souhaité partager la souffrance des jeunes interviewés avec différents acteurs et actrices de la société (parents, associations de migrant·e•s, professionnel-le.s du social et de la santé, services publics concernés par les migrations) par un travail de restitution des résultats de recherche. Cette restitution était très importante parce qu'elle offrait aux enquêté·e.s «un espace-temps d'appropriation critique leur permettant non seulement de prendre connaissance de la production scientifique mais de faire entendre leurs voix" (Bergier, 200I: I9I). Ma démarche s'inscrivait également dans une approche citoyenne de restitution (Olivier de Sardan, 2004), qui offre de débattre publiquement sur une problématique sociale.

La restitution des résultats de ma recherche sur les jeunes Rwandais·e.s a été menée en France et en Suisse. J'ai notamment présenté les résultats de la recherche aux membres de la communauté rwandaise en Suisse en mai 2003 (une année avant de terminer ma thèse) dans le cadre des activités socioculturelles d'une association rwandaise. La majorité des jeunes interviewés en Suisse ont assisté à cette conférence et ont pu donner leurs opinions sur les résultats de la recherche. Pour ces jeunes, la conférence a été une occasion de partager leurs problèmes et leurs 
ressources avec le public et les autres jeunes qui n'avaient pas participé à l'étude. Cette conférence a incité les jeunes Rwandais·e.s de Suisse à organiser, le 2I février 2004 - au moment où je terminais ma thèse -, une conférence-débat intitulé Les jeunes Rwandais en Suisse: perspectives d'avenir. Elle a mobilisé une trentaine de jeunes Rwandais·e.s, y compris ceux venus de Belgique, pour parler de problèmes liés à la culture, à la famille et à l'intégration. Les éléments discutés et les propositions faites par les jeunes ont confirmé les résultats de ma recherche, portant notamment sur la souffrance psychologique des jeunes Rwandais·e•s ayant vécu des événements traumatiques, le rôle de la communauté d'origine en exil dans le processus de résilience et l'importance de l'école pour s'intégrer dans la société d'accueil. D'autres conférences, auxquelles des professionnel.le.s ont participé, ont été organisées en France et ont permis de mieux comprendre les besoins des jeunes Rwandais·e·s réfugié.e.s pour leur prise en charge psychosociale. Les résultats de mon étude ont également fait l'objet de certaines publications (Gakuba, 2004; 2005; 2013) dans lesquelles j'ai intégré certains éléments issus des discussions lors de la restitution des résultats.

Concernant l'étude sur le processus d'intégration des jeunes issus de l'immigration africaine subsaharienne, la restitution des résultats a été faite à travers des présentations dans le cadre de conférences scientifiques ou événements organisés par des associations genevoises impliquées dans l'accompagnement des jeunes migrant.e.s. J'ai à chaque fois pris le soin d'inviter les jeunes ou les responsables d'associations et structures impliquées dans la recherche. J'ai, par exemple, présenté les résultats de la

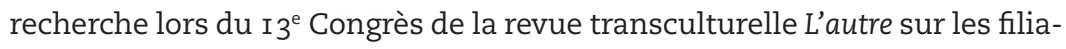
tions, affiliations et adoptions, qui a eu lieu les 2 et 3 décembre 20I I, aux Hôpitaux universitaires de Genève. Ma communication, intitulée «Identités, appartenances culturelles et nationales ", a porté sur les jeunes Suisses d'origine africaine en montrant les différentes appartenances nationales évoquées par les jeunes:

, les jeunes qui acceptent leur double ou multiple appartenance culturelle et nationale;

, les jeunes qui se définissent comme étrangers en Suisse et étrangers dans le pays d'origine;

, les jeunes Suisses d'origine africaine, mais qui se définissent comme étrangers en Suisse. 
Les professionnel-le.s accompagnant les jeunes migrant.e.s qui étaient venus assister à ma présentation ont estimé que cette typologie pouvait faire l'objet d'une réflexion sur comment accompagner les jeunes dans leurs projets d'avenir en faisant le lien avec les représentations de leur identité.

D'autres présentations ont été données dans le cadre d'événements organisés par des associations africaines et de migrant.e.s à Genève. Je peux citer l'atelier organisé à Zurich en 2012 par le réseau des jeunes Africains de la diaspora en Suisse. Les jeunes participant à l'atelier m'ont confié que les résultats de ma recherche leur avaient permis de mieux se connaître, d'être sensibles aux difficultés d'intégration rencontrées par les jeunes. Enfin, la restitution des résultats de la recherche a été faite lors d'un caucus africain organisé par des associations africaines à Genève, en octobre 2016, et lors du Forum des étrangers, organisé par des associations de migrant.e.s à Genève en mars 2017. Des professionnel.le.s, des membres de la communauté africaine et des jeunes ayant participé à ces événements ont échangé sur des recommandations proposées, au sujet de l'accompagnement des jeunes migrant.e.s d'Afrique subsaharienne.

\section{Conclusion}

Dans cet article, j'ai montré comment les conditions de vie des personnes migrant.e.s, l'histoire personnelle de ces dernières et leurs appartenances identitaires sont parmi les causes de la souffrance psychologique exprimée par les jeunes dans les entretiens de recherche. Face aux différents témoignages traduisant cette souffrance des personnes interviewées, le ou la chercheur.e éprouve un malaise (Rostaing, 2010) qui l'amène à adopter certaines postures dans la relation d'entretien. Dans le cas de jeunes migrant.e.s qui ont vécu des événements traumatisants, l'entretien peut être un espace où exprimer des traumatismes. Les chercheur·e.s doivent ainsi faire preuve d'écoute et d'empathie pour comprendre la souffrance de ces jeunes, mais ils ne peuvent, du fait de leur position (et de leur manque de formation), entamer un travail de suivi psychologique ou autre. Cette situation exige une bonne préparation des entretiens, en tenant compte de tous les aspects éthiques (confidentialité, consentement libre, respect de la sphère privée) et des répercussions que ces entretiens pourraient avoir sur les personnes interviewées. Dans des situations d'entretiens 
problématiques sur le plan affectif et émotionnel, une prise en charge des personnes interviewées par les professionnel.le·s agréé.e.s peut être conseillée. Il est aussi, d'ailleurs, important de noter que les chercheur.e.s qui sortent affecté.e.s par les entretiens difficiles avec des personnes en souffrance puissent bénéficier de séances de supervision dans lesquelles il soit possible de parler de ses propres expériences, difficultés et limites. Dans mon cas, l'école doctorale que je suivais était un lieu approprié pour partager mes expériences et où je pouvais trouver un soutien durant les moments difficiles de la recherche.

Pour d'autres jeunes migrant.e.s, l'entretien peut être considéré comme une occasion pour faire valoir des revendications ou parler des injustices et des frustrations vécues dans le pays d'accueil. Les propos, d'une certaine virulence, peuvent embarrasser les chercheur.e, du fait qu'ils ne peuvent pas agir directement, ni psychologiquement ni politiquement, sur ces frustations. Avant de commencer l'enquête de terrain, il est important pour les chercheur.e.s de faire un travail de réflexivité (Chimienti, 20Io) sur l'objet de la recherche et sur la population étudiée; ce qui pourrait permettre aux chercheur.e.s d'anticiper les demandes qui pourraient être exposées dans les entretiens par la population étudiée. Les jeunes, et plus particulièrement celles ou ceux qui sont dans un processus de demande d'asile, peuvent percevoir les chercheur.e.s comme des personnes qui pourraient améliorer leurs conditions de vie. Des demandes sociales (recherche d'emploi, apprentissage, permis de séjour) peuvent, lors de l'entretien, leur être adressées. L'impossibilité d'accéder à ces demandes doit être clarifiée au début de la recherche; le ou la chercheur.e évitera de se positionner dans une situation de relation d'aide. Cependant, les chercheur.e.s doivent être conscient.e.s de leur responsabilité dans la reviviscence des traumatismes lors d'entretiens avec des personnes ayant vécu des événements traumatiques, et peut proposer aux enquêté.e.s vulnérables des informations sur les structures existantes de prise en charge psychosociale des personnes migrantes.

Enfin, il nous paraît indispensable d'envisager la restitution des résultats de la recherche sur les jeunes migrant.e.s qui ont connu des parcours migratoires difficiles pour faire connaître leur souffrance. C'est par la restitution que les actrices et acteurs de la société (jeunes, parents, associations de migrant.e.s, professionnel.le.s, services publics concernés par les migrations, associations d'aide aux migrant.e.s) auront une meilleure connaissance de la situation de ces jeunes pour une meilleure prise en charge psychosociale et une bonne intégration dans la société d'accueil. 


\section{Références bibliographiques}

Bagalwa, J. \& Bolzman, C. (20I4). Accès à l'emploi qualifié: difficultés rencontrées par les diplômés d'origine africaine. Tangram, 33, 84-87.

Bergier, B. (200I). Repères pour une restitution des résultats de la recherche en sciences sociales. Paris: L'Harmattan.

Boutin, G. (I997). L'entretien de recherche qualitatif. Québec: Presses de l'Université du Québec.

Chimienti, M. (20I0). Le «stigmate de putain". Les défis posés par la relation d'enquête auprès de travailleuses du sexe migrantes. Dans J.-P. Payet, C. Rostaing \& F. Giuliani (Ed.), La relation d'enquête. La sociologie au défi des acteurs faibles (pp. 39-55). Rennes: Presses universitaires de Rennes.

Cyrulnik, B. (Ed.) (I998). Ces enfants qui tiennent le coup. Revigny-sur-Ornain: Hommes et perspectives.

Demazière, D. (20I2). L'entretien de recherche et ses conditions de réalisation. Variétés des sujets enquêtés et des objets de l'enquête. Sur le journalisme, I(I), 30-39.

Decety, J. (2004). L'empathie est-elle une simulation mentale de la subjectivité d'autrui? Sciences humaines, I50, 54-88.

Eyer, P. \& Schweizer, R. (2010). Les diasporas somalienne et érythréenne en Suisse. Berne: Office fédéral des migrations.

Gakuba, T.-O. (2004). La résilience des jeunes Rwandais réfugiés en France et en Suisse [Thèse de doctorat en Sciences de l'éducation]. Genève: Université de Genève.

Gakuba, T.-O. (2005). Sentiment de métamorphose identitaire chez les jeunes Rwandais réfugiés en Europe. Diversité-Ville Ecole Intégration, I43, I35-I40.

Gakuba, T.-O. (20I3). Integration process of young sub-Saharian African migrants in Switzerland. International Network on Youth Integration Journal, 3(2), 4-5.

Haute école de travail social de Genève (2009). Code d'éthique de la recherche. Genève: HETS.

Haas, V. \& Masson, E. (2006). La relation à l'autre comme condition à l'entretien. Les Cahiers Internationaux de Psychologie Sociale, 7I, 77-88

Legavre, J.-B. (1996). La «neutralité» dans l'entretien de recherche. Retour personnel sur une évidence. Politix, 9(35), 207-225.

Olivier de Sardan, J.-P. (1995). La politique du terrain. Consulté le I0.0I.2018 sur http://enquete.revues.org/263; DOI: I0.4000/enquete. 263

Piguet, E. (2004). L'immigration en Suisse. Cinquante ans d'entreouverture. Lausanne: Presses polytechniques et universitaires romandes.

Poupart, J., Deslauriers, J.-P., Groulx, L. H., Laperrière, A., Mayer, R. \& Pires, A. P. (I997). La recherche qualitative. Enjeux épistémologiques et méthodologiques. Montréal: Gaëtan Morin.

Pourtois, J.-P. \& Desmet, H. (I988). Epistémologie et instrumentation en sciences humaines. Liège: Mardaga.

Rosenberg, M. (I986). Self-concept from middle childhood through adolescence. Dans J. Suls \& A.G. Greenwalds (Ed.), Psychological perspectives on the self (vol.3, pp. I07-I36). Hillsdale: Lawrence Erlbaum. 
Rostaing, C. (20I0). On ne sort pas indemne de prison. Le malaise du chercheur en milieu carcéral. Dans J.-P. Payet, C. Rostaing \& F. Giuliani (Ed.), La relation d'enquête. La sociologie au défi des acteurs faibles (pp. 23-37). Rennes: Presses universitaires de Rennes.

Sanchez-Mazas, M. \& Roca I Escoda, M. (2007). La dimension politique de la reconnaissance: clés de lecture de l'action collective à partir de la théorie d'Axel Honneth. Viure ensemble au XXe siècle. [Actes du colloque international de l'Institut de sociologie]. Bruxelles: Publications de l'Institut de sociologie.

Sanchez-Mazas, M., Maggi, J. \& Roca I Escoda, M. (20I0). En quête de la voix des sansdroits. Le cas des exclus du droit d'asile. Dans J.-P. Payet, C. Rostaing \& F. Giuliani (Ed.). La relation d'enquête. La sociologie au défi des acteurs faibles (pp. I43I60). Rennes: Presses universitaires de Rennes.

Vanistandael, S. \& Lecomte, J. (2000). Le bonheur est toujours possible. Construire la résilience. Paris: Bayard. 
Recherche et enseignement

Deuxième partie 



\title{
La recherche anthropologique dans l'enseignement en travail social
}

\author{
Laurence Ossipow
}

\begin{abstract}
A Sabine Voélin et Gaëlle Aeby, qui ont enseigné en même temps que moi la sociologie et l'anthropologie dans le module C4, ainsi qu'à Milena Chimienti et Anne Lavanchy, qui ont repris et développé avec brio ces enseignements lorsque j'ai dû les abandonner pour d'autres responsabilités.
\end{abstract}

\section{Introduction}

Des projets de recherche ont toujours été menés depuis la création du CERES (Centre de recherche sociale) en I968. Toutefois, depuis que l'Institut d'études sociales (IES) s'est transformé en 2004 en Haute école de travail social (HETS) liée à la HES-SO Genève et aux autres HES de Suisse occidentale, la recherche s'est affirmée comme une des missions de la HETS et constitue une part entière de l'enseignement. Ainsi, au moment où le Rectorat et les directrices ou directeurs d'établissement souhaitent apporter la preuve que la recherche est bien incluse dans les cours en HES, il semble nécessaire de rappeler comment apport théorique général, description de recherches spécifiques et posture d'enseignement peuvent s'articuler. A partir d'un exemple précis, le module Relations à l'autre, individuelles et collectives, qui laisse place aussi à la psychologie et à des séances plus spécifiques sur la pratique de l'entretien individuel et collectif, je montrerai comment j'ai élaboré cet enseignement dans le cadre du module, en tenant notamment compte de ce qui était déjà développé en cours de psychologie, une série de cours davantage centrés sur la relation à l'autre dans le cadre du couple parental et des relations individuelles. Pour ce faire, j'ai choisi certains concepts qui m'apparaissaient importants en anthropologie et particulièrement adaptés pour favoriser la réflexivité en travail 
social, et présenté plusieurs recherches - y compris les miennes - comme exemples ou bases d'exercices. Le choix de chaque concept sera expliqué et décrit, puis fera l'objet d'un commentaire sur les réactions des étudiant.e.s appelé.e.s à s'en saisir. En conclusion, je soulignerai le nécessaire lien entre enseignement et recherche et me demanderai comment en améliorer les conditions de transmission et de discussion.

\section{Identité et altérité}

Quand j'ai commencé à préparer mon enseignement dans la partie anthropologie et sociologie du module Relations à l'autre, individuelles et collectives (dont j'étais aussi la responsable), alors que je venais d'être fraichement nommée à la HETS après des années d'enseignement en anthropologie à l'Institut d'ethnologie de l'Université de Neuchâtel, je me suis demandé comment faire pour transmettre aux étudiant.e.s un cadre théorique fondé sur cette discipline qui m'apparaissait être au cœur de la question de l'identité et de l'altérité ${ }^{1}$ et comment déployer un enseignement qui suscite la réflexion et la réflexivité. Me souvenant des concepts qui avaient été très importants dans mes propres études en anthropologie, dans le cadre d'un poste à l'Hospice général (l'institution qui gère l'aide sociale à Genève) et dans les recherches que j'avais menées, j'ai pensé qu'il ferait sens de fonder le cours sur quatre grands thèmes: «l'échange et la réciprocité»; «la distinction par l'habitus»; «la culture» et «les rites».

J'ai d'abord cherché à traiter du thème des échanges et de la réciprocité, qui sont au cœur du lien social. Ce thème me semblait d'autant plus important que les discussions, autant à l'Hospice général qu'à la HETS, sur l'effritement du lien social étaient nombreuses. Il était donc intéressant de comprendre comment ces liens se construisent avant de, peutêtre, se déliter... Même si la question des échanges thématisait déjà celle des enjeux de pouvoir, il m'apparut qu'il fallait aussi initier les étudiant.e.s aux processus de distinction et de domination liés aux classes sociales. C'est ainsi que je choisis de me concentrer sur la notion de socialisation par le biais de celle de l'habitus. Ce choix avait notamment pour objectif de contrebalancer la vision très psychologisante que les étudiant.e.s semblaient avoir des relations sociales, ainsi que me l'affirmait une collègue qui avait déjà enseigné dans ledit module. Echange, réciprocité, habitus et distinction de classes, voilà des bases pour comprendre certains processus sociaux, mais quid de la culture? Cette notion phare en anthropologie 
constitue aussi une problématique importante pour les travailleuses et travailleurs sociaux en lien avec la migration. La notion de culture permit alors de reprendre l'analyse de l'espace social sous un angle plus interactionniste que le point de vue emprunté par Pierre Bourdieu. Enfin, la thématique des rites s'imposait, non seulement parce que les rites font et défont les liens sociaux, mais aussi parce qu'il était intéressant de montrer aux étudiant.e.s que les rites existent ici aussi, et non pas seulement dans les sociétés dites exotiques. Par ailleurs, le thème des rites permettait de reprendre d'une autre façon une bonne partie des auteur.e.s classiques abordé.e.s précédemment au cours des autres séances thématiques, donc de revenir sur leur approche théorique, mais sous un autre angle.

\section{Les thèmes abordés}

\section{L'échange et la réciprocité}

«Refuser de donner, négliger d'inviter, comme refuser de prendre équivaut à déclarer la guerre, c'est refuser l'alliance et la communion " (Mauss, 200I [I950]: I62-I63). Pour travailler sur la question de l'échange, il fallait bien sûr renvoyer aux travaux de Marcel Mauss (200I [I950]), repris et commentés par de nombreux chercheur·e•s, notamment Claude LéviStrauss (I967 [I949]; 200I [I950]), qui cherchait à montrer que les sociétés (les hommes) "échangent des femmes "², non pas pour éviter un éventuel risque d'inceste au sein d'un même groupe de parenté, mais pour mettre en évidence que ces échanges créent des alliances, gages de communication, de paix et de collaboration plutôt que de guerres intestines. La

1 Pour une anthropologue, le titre du module faisait en effet référence directe à la notion d'identité, qui ne peut pas être conçue isolément, l'identité (ou l'autodéfinition) d'une personne ou d'un groupe se définissant d'emblée dans les relations entretenues avec d'autres personnes ou groupes qui imposent ou tentent d'imposer leurs propres définitions dans un contexte historique et social spécifique et dans la saillance des interactions (voir infra le troisième point, «La culture»).

2 Le fait que Lévi-Strauss explique que ce sont «des hommes qui échangent des femmes» a été longuement discuté dès les années I960. Sur ce point, lire notamment Collard (2000), qui s'efforce de décrire un petit nombre de sociétés dans lesquelles ce sont les femmes qui organisent les alliances. 
présentation des théories de Marcel Mauss, elles-mêmes fondées sur les enquêtes de terrain de Franz Boas et Bronislaw Malinowski (voir plus loin, infra), avait aussi pour objectif de montrer que l'échange marchand n'était pas à la base de toutes les relations comme si l'être humain n'était qu'un homo economicus. Ne serait-ce que pour obliger les étudiant.e.s à décentrer leur regard, je choisis de présenter en détails les exemples célèbres et très exotiques du kula et du potlatch à travers textes et films. Le potlatch, terme issu du sabir chinook (une langue de traite datant de la fin du I $^{\mathrm{e}}$ siècle, apparue sur la côte nord-ouest de l'Amérique du Nord, notamment chez les Kwakiutl), étudié par Franz Boas, anthropologue américain d'origine allemande, signifie «don" ou «donner», "nourrir» et "consommer» dans un contexte cérémoniel. Il définit un ensemble de manifestations (fête, danse, alliance, discours, distribution ostentatoire de biens de prestige) autrefois pratiquées pendant l'hiver par les peuples pêcheurs-chasseurs-cueilleurs de la côte nord-ouest pour acquérir et maintenir une influence politique et une position sociale au sein d'un système politique de rangs (Mauzé, I986)33. Le potlatch ratifie à la fois le statut de donateur et celui de donataire. S'il ne veut pas être déshonoré, le donataire doit rendre au moins l'équivalent de ce qui a été reçu au cours d'un potlatch qu'il organisera ultérieurement. Le kula, décrit, en 1922, par le célèbre anthropologue anglais Bronislaw Malinowski (I963 [I922]) pour le Pacifique et la Micronésie, pourrait paraître comme une notion encore plus déstabilisante que celle du potlatch dans la mesure où le cycle d'échanges qui le caractérise repose sur le transfert alterné de bracelets et colliers de coquillages dont la valeur n'est ni utilitaire ni décorative, mais strictement cérémonielle (Jeudy-Ballini, 2004). Le kula peut être considéré, pour reprendre l'expression de Marcel Mauss, comme un "phénomène social total» parce qu'il met en branle toutes les institutions et tous les domaines d'une société (échanges économiques et symboliques, liens affectifs, techniques matérielles, art, religion, convivialité, etc.). Les étudiant·e.s ne furent qu'à moitié étonné.e.s par ces échanges quasi inutiles en apparence et trouvèrent des exemples quelque peu comparables dans nos sociétés occidentales, par exemple les trophées de football qui passent d'un joueur à l'autre pour revenir à la buvette du club ou les petits cadeaux, parfois des broutilles, qu'échangent des ami.e.s juste pour maintenir un lien. D'autres auteur.e.s permirent de prolonger la réflexion sur l'échange en montrant les différentes formes de réciprocité qui pouvaient s'établir entre groupes et sociétés (Sahlins, I976) ou entre individus. Je décidai aussi de m'arrêter en particulier sur un texte d'Alain Testart (200I), qui 
opère une distinction entre don, échange non marchand et échange marchand. Je pensais utile d'expliquer cette différence aux étudiant.e.s car, dans la recherche que nous étions parallèlement en train de mener sur l'aide sociale octroyée par l'Hospice général (Ossipow, Lambelet \& Csupor, 2008), elle se révélait très utile. La distinction conceptualisée par Testart permettait en effet de faire comprendre à la fois les cadeaux qui s'échangeaient parfois entre actrices et acteurs de l'aide sociale et le "contrat»-dénommé le $\mathrm{CASI}^{4}$ au moment de sa mise sur pied - passé, vers la fin de notre enquête en 2007, entre l'Hospice général et les bénéficiaires. En référence à la théorisation de Testart, nous avons qualifié ce CASI d'«échange contractuel» (Ossipow, Lambelet \& Csupor, 2008). Les cadeaux que nous avons recensés dans cinq centres d'action sociale (CASS) à Genève étaient des dons initiés par les assistant·e.s sociales et sociaux ou les bénéficiaires et ne servaient à rien ou presque rien, si ce n'est à maintenir la relation autrement que dans son strict côté utilitaire ou hiérarchique, tandis que le contrat, lui, constituait un échange, mais un échange non marchand, dans la mesure où, même si ce contrat pouvait déboucher sur une sanction qui diminuerait le montant de l'«entretien» mensuel touché comme aide sociale, il ne s'agissait pas d'argent investi sur le marché économique ni d'une relation avant tout déterminée par l'achat et la vente, ce qui est le cas de l'échange marchand au sens strict du terme. En examinant nos données de terrain de recherche avec les collègues et en discutant en cours avec les étudiant.e.s, qui me donnaient beaucoup d'exemples rencontrés sur leurs propres terrains d'intervention ou de stage, nous nous sommes rendu compte que toutes les formes de cadeaux offerts ou reçus dans le cadre de l'aide sociale ressemblaient aux dons que les anthropologues recensent dans les processus de kula ou potlatch (des dons avant tout collectifs et non individuels). Leur caractéristique commune était la notion de défi: montrer que l'on n'était pas une personne pauvre, qui serait incapable du moindre don,

3 Pour une discussion de ces pratiques, dont on a pu dire que leur côté extrêmement ostentatoire tenait au fait que les sociétés qui les organisaient étaient en crise, lire également Mauzé (I986). L'acronyme désigne un contrat d'aide sociale individuel. La notion même de contrat était discutée au moment de sa mise sur pied puisque celui-ci ne résultait pas vraiment d'un engagement volontaire et d'un accord assumé entre deux personnes disposant du même pouvoir, mais concernait des bénéficiaires quasiment obligé.e.s de s'y soumettre. 
remercier pour le travail «en plus» ou le temps «en plus» offert par le ou la professionnel.le, offrir un objet qui dit quelque chose de son origine (une bouteille de porto, de l'artisanat d'Amérique latine...) ou de ses compétences (un cadre avec une photographie d'auteur.e, des biscuits faits maison, etc.). Comme le souligne Marcel Hénaff (2002), revisitant notamment les théories de Marcel Mauss, donner et accepter de recevoir, c'est accepter de s'engager dans un processus de reconnaissance mutuelle. Pour clore la partie de ce cours, nous avons choisi de faire travailler les étudiant.e.s sur la question de la reconnaissance. Nous avons alors emprunté tant aux travaux d'Axel Honneth (2000) qu'à ceux de Nancy Fraser (2005), qui associe plus directement qu'Honneth la question de la reconnaissance à celle de la justice sociale.

\section{La distinction par l'habitus}

Pour traiter de l'habitus et de l'espace des classes sociales, j'ai eu recours aux concepts développés par Pierre Bourdieu dans La Distinction. Critique sociale du jugement (I979). J'avais lu par le menu ce livre pour discuter dans ma thèse (Ossipow, I997) les changements de style de vie (et peut-être conséquemment d'habitus) des personnes quittant un régime omnivore pour adopter une diète végétarienne, végétalienne, crudivore ou macrobiotique. Le modèle théorique de l'habitus construit par Bourdieu peut se définir comme un système plus ou moins durable de dispositions incorporées au cours d'une biographie socialement et historiquement située. Ces dispositions informent les perceptions et les pratiques des personnes dans des situations et contextes variés. Dans La Distinction (I979), Bourdieu met en correspondance l'espace des conditions sociales et l'espace des styles de vie par l'intermédiaire de l'habitus. Chaque individu et classe ou fraction de classe se définit par une certaine structure de ses capitaux: le «capital économique», le «capital culturel» (de formation) et le «capital social» (de relations), les trois étant susceptibles d'être convertis en "capital symbolique». La classe à laquelle appartient une personne est alors définie, non seulement à travers des indices comme la profession, le revenu ou le capital scolaire, mais aussi par un ensemble de caractéristiques liées aux autres capitaux et aux relations entre ceux-ci. Comme on peut le voir sur le tableau, Bourdieu (I979: I9I) montre bien aussi comment un habitus et un style de vie sont en contraste avec d'autres habitus et styles de vie. A ces deux notions doit s'ajouter celle de champ, si l'on veut comprendre la 
force des propositions bourdieusiennes. La structure d'un champ est un état du rapport de forces entre les agent.e.s ou les institutions engagées dans la lutte avec leurs capitaux spécifiques. Ainsi, chaque champ (le champ de la culture, des luttes ouvrières...) a ses spécificités, son histoire, ses espaces physiques et symboliques, ses acteurs et ses actrices, ${ }^{5}$ ses rapports de force, ses stratégies et ses transformations. Même si certaines frontières se craquellent et certains comportements s'homogénéisent en deçà ou au-delà des classes sociales, les hiérarchies et les dominations se maintiennent dans le temps. Ainsi, les dominant.e.s vont tout mettre en œuvre pour se maintenir à leur place tandis que les dominé·e.s, sauf exception, acceptent cet état de fait sans forcément s'en rendre compte. Cette reproduction des inégalités réduit la mobilité sociale, autrement dit le mouvement vers le haut des classes dites dominées. En démontant et démontrant ce jeu social, Bourdieu a, en quelque sorte, libéré de nombreuses personnes qui pensaient que leurs difficultés de progression sociale étaient dues à leurs incompétences individuelles, à leur absence de charisme, et qui ont découvert que ce dont elles souffraient était soit un manque d'informations, de stratégies, soit l'absence d'un réseau social susceptible de leur permettre certains accès, notamment sur le marché du travail. La question de la détermination des biographies par l'habitus a été relativisée, notamment grâce aux travaux de Bernard Lahire (I998) et à ceux, bien plus anciens, de Peter Berger et Thomas Luckmann (I986 [I96I]), qui permettaient de montrer comment des expériences «totalisantes "6 pouvaient ouvrir sur une resocialisation et laisser place à des conversions ou retournements (des alternations) propres, non pas forcément à changer d'habitus, mais en tout cas à s'en distancier.

\section{La culture}

La notion de culture offrait une autre façon de considérer les rapports de force dans l'espace social et l'occasion de parcourir l'histoire de l'anthropologie à partir d'un seul concept. C'est pourquoi La notion de culture

5 Ou «agent.e.s», dit Bourdieu, puisqu'ils et elles sont potentiellement moins libres que des «acteurs" et "actrices».

6 Par exemple, une conversion religieuse ou le choix d'une pratique alimentaire qui entraîne petit à petit d'autres changements. Sur ce dernier point, voir Ossipow (1997). 
dans les sciences sociales, le petit livre (mais constamment réédité) de Denys Cuche (I996), fut donné en lecture obligatoire pour l'ensemble du cours. La visite de l'exposition «Nous autres", mise sur pied en 2005 au Musée d'ethnographie de Genève, fut aussi l'occasion de traiter du thème de l'identité et de l'altérité à travers une autre pédagogie, celle de l'exposition muséographique. L'objectif du cours sur la culture consistait à montrer que la notion - même si elle renvoyait à celle, considérée comme très positive, de diversité - était discutable, presque une manière politiquement correcte de remettre la notion de race au goût du jour, ainsi que l'avait montré Colette Guillaumin (I98I ; I992) dans plusieurs articles, y compris dans celui republié dans le classeur pédagogique édité par Monique Eckmann et Michèle Fleury (2005) sur une approche de la question du racisme et de la citoyenneté dans une perspective théorique, mais aussi orientée vers l'action pour les professionnel.le.s (pour d'autres exemples, voir aussi Eckmann, Sebeledi, Bouhadouza \& Wicht, 2009).

Après avoir montré comment la culture pouvait référer à des attachements "primordiaux" et à des «besoins" émotionnels (liens de parenté, force de la solidarité), à un sentiment d'affinité naturelle, à l'importance des loyautés familiales ou locales (liens du sang, traits phénotypiques, religion, langue, appartenance régionale) et à l'indicible de l'entre-soi, incommunicable aux outsiders (Poutignat \& Streiff-Fenart, I995: 93-I32), je m'efforçai de convaincre les étudiant.e.s que les liens familiaux et/ou régionaux, même s'ils sont incontournables, ne devaient pas être sur-considérés, au risque de figer les personnes dans leurs appartenances premières. Autrement dit, l'identité "culturelle» ne devrait pas être considérée comme supplantant toutes les autres formes d'identité et, surtout, elle se fonde sur des processus d'identification (ou auto-attribution) et d'assignation (exoattribution) variant selon les contextes, les rapports de force et de pouvoir ainsi que les individus ou groupes en présence. C'est d'ailleurs ce que montrait le texte de Fredrick Barth (I969), traduit par Poutignat et StreiffFenart en I995. Comme l'ont rappelé par la suite Anne Lavanchy, Anahy Gajardo et Fred Dervin (20I I : I9-20), les différences culturelles n'ont pas «le caractère de données tangibles et réelles » et elles n'existent pas dans l'absolu ni «en dehors de tout système social et cognitif».

Pour tenter d'expliquer au mieux l'importance du contexte et de la saillance des interactions, je fis lire l'exemple d'Anatole, relaté dans l'ouvrage de Poutignat et Streiff-Fenart (I995: I85). Cet extrait raconte comment un étudiant de nationalité burkinabè circulant dans un bus niçois 
se voit donner l'ordre de présenter sa carte de réduction demi-tarif en sus du ticket qu'il a composté. Le contrôleur, apparemment excédé que des usagères et usagers présumé.e.s fraudeur.e.s, le prennent de haut, associe cet étudiant aux «clandestin.e.s cap-verdien.ne·s» (comme le rapportent les deux auteur·e·s) ayant immigré à Nice et victimes de nombreux préjugés. Il menace ensuite l'étudiant de devoir payer une amende. Ce dernier conteste le fait d'être amendable puisqu'il a simplement oublié l'étui qui contient à la fois son permis de résidence et sa carte de réduction. Devant ce refus d'obtempérer, le contrôleur prétend pouvoir appeler la police, ce que l'étudiant accepte posément. En fait, expliquent les auteur.e.s du récit, le contrôleur tente de définir la situation à sa façon en s'appuyant sur les stéréotypes liés aux migrant.e.s critiqué.e.s à Nice. Comme le font remarquer Poutignat et Streiff-Fenart (I995), le contrôleur sait probablement que l'étudiant n'est pas cap-verdien ou migrant récemment arrivé (notamment par sa parfaite maitrise du français), mais il utilise le stéréotype. De son côté, l'étudiant, sûr de son bon droit, n'accepte pas d'être accusé, ni assimilé aux Cap-Verdien-ne-s du simple fait de sa couleur de peau. Il maintient une ligne de défense que n'importe quelle usagère ou usager utiliserait dans pareil cas. Les réactions des autres passagères et passagers du bus ne sont pas décrites, mais on peut facilement les imaginer, prenant parti pour l'un ou l'autre des protagonistes. Cet exemple laisse comprendre comment les assignations et les identifications se font en contexte, dans un jeu de pouvoir et de résistance, en lien avec des stéréotypes reliés à des rapports de domination et de prétendue légitimité sur un sol national.

\section{Les rites}

Avec la quatrième et dernière notion, celle de rite, je voulais montrer aux étudiant.e.s comment les processus rituels renouvelaient de diverses façons le lien social, même s'ils avaient aussi pour objectif de critiquer la société, voire de s'inscrire en rupture avec les structures conventionnelles. Les ouvrages classiques traitant du rite ont donc été présentés: des premiers travaux d'Arnold van Gennep sur les rites de passage (I98I [I909]) aux écrits d'Emile Durkheim sur les structures du religieux (2003 [I9I2]), en passant par ceux de Victor Turner sur la place des rites dans et hors des structures sociales habituelles (I969). Je revins aussi sur les travaux de Pierre Bourdieu, notamment sur un de ses textes, "Les rites comme actes d'institution" (I982), qui soutient que les rites marquent moins un passage 
qu'une ligne de démarcation arbitraire qui permet de séparer «ceux qui ont subi [le rite] non de ceux qui ne l'ont pas encore subi, mais de ceux qui ne le subiront en aucune façon et d'instituer ainsi une différence durable entre ceux que ce rite concerne et ceux qu'il ne concerne pas» (I982: 206). Par exemple, le rite d'institution sépare celles et ceux qui peuvent "subir» le rite de naturalisation de celles et ceux qui ne le subiront pas car ne comptant pas encore le nombre d'années de résidence légale leur permettant de déposer une demande d'acquisition de la nationalité suisse. La diversité des rites contemporains et occidentaux fut aussi déployée à l'aide de différents exemples: par exemple, celui de Claude Lévi-Strauss (I952) décrivant la fête de Noël à Dijon en I95I, lorsqu'une effigie du père Noël est brûlée devant la cathédrale par des catholiques pour dénoncer un rite laïc en train de devenir à la mode après-guerre sous l'influence des Américain·e•s. Par exemple aussi, celui du mariage expliqué par Martine Segalen (2005), qui, en référence au folkloriste et ethnologue van Gennep, montre les différentes phases du rite, du moment de la séparation du monde antérieur par les fiançailles jusqu'à la réintégration des fiancé.e.s dans leur nouveau statut de personnes mariées. Des exemples furent également donnés sur les rites politiques contemporains expliqués par Claude Rivière (I995, I998) et Marc Abélès (voir notamment 2007), ou encore sur les rites d'adolescence décrits par Michel Bozon (2002) comme des «rites de première fois».

\section{Réactions et retours}

L'ensemble de notions et concepts décrits ci-dessus ont fait l'objet de présentations théoriques ex cathedra, de séminaires de lecture et d'exercices d'analyse de données de recherche. Dans les séminaires, c'était aux étudiant·e.s (et non plus à l'enseignant·e) de présenter des textes et de les expliquer à leurs camarades.

Les étudiant.e.s semblèrent apprécier la première thématique, celle de l'échange, des dons et des contre-dons, même si, ou peut-être parce que les exemples y étaient d'abord tirés de contextes exotiques (voir le potlatch et le kula évoqués plus haut), mais qu'ils leur apparaissaient malgré tout assez faciles à ramener à leur propres expériences pour comprendre comment le système de la réciprocité fonctionnait dans nos sociétés occidentales. Maint.e.s étudiant.e.s référèrent à des exemples qu'ils tiraient de leurs pratiques passées ou actuelles dans différents lieux du travail 
social: maisons de quartier, lieux de distribution de l'aide sociale, suivi d'adolescent.e.s et jeunes adultes en travail social hors murs ou dans des foyers éducatifs, centres de jour ou établissements pour personnes âgées, etc. Un étudiant a, par exemple, longuement expliqué, en plein auditorium et gestes à l'appui, comment, travaillant dans un EMS avec des personnes souffrant de troubles cognitifs, une résidente lui avait donné une pièce de deux francs suisses «sans la lui donner vraiment» (elle n'en avait pas dans la main), mais en lui faisant comprendre le don par un signe qui indiquait de la mettre dans sa poche sans la montrer aux autres. Une belle façon de souligner, à la fois le lien qu'elle entretenait avec l'étudiant et le défi que représentait un don d'argent, bien sûr interdit dans le cadre des relations professionnelles et du travail social en particulier. Et comme il s'agissait d'un don imaginaire (ce qui ne veut pas dire inexistant), ce fut aussi l'occasion de revenir sur l'importance du symbolique dans les relations sociales. Cependant, c'est probablement mes collègues Milena Chimienti et Anne Lavanchy qui ont su le mieux faire comprendre aux étudiant.e.s la part de défi qu'il y a dans tout don en présentant à leur tour les notions de potlatch et de kula évoquées au point «L'échange et la réciprocité» (voir supra). Les étudiant.e.s, organisant une fête à la HETS pour leur association, élaborèrent en effet une affichette imitant un masque de danse potlatch suivi de l'incitation "Ramène ton don!", une incitation à donner qui n'est pas ainsi dite explicitement dans les cérémonies de potlatch, mais fait intimement partie du processus d'échange et de réciprocité dans lequel les participant.e.s mettent en jeu leur honneur. Ne rien «ramener» ou offrir correspondrait ainsi au déshonneur des étudiant·e.s vis-à-vis de leurs pairs et de l'association organisatrice. Peut-être même au refus de la convivialité, dont on sait qu'elle est une forme particulièrement importante des échanges sociaux.

Pour le concept d'habitus, les textes furent plus difficiles à trouver, car souvent intégrés à des recherches dont la lecture se révélait difficile, probablement en raison du cadre théorique complexe mobilisé autour des travaux de Pierre Bourdieu. Le roman autobiographique d'Annie Ernaux La place permit en revanche de montrer d'une autre façon, en faisant davantage appel aux émotions qu'aux raisonnements, comment on peut avoir le sentiment de ne pas être à sa place dans un monde d'enseignant.e.s et d'écrivain.e.s lorsqu'on est issu de classes dites populaires. Il n'en restait pas moins que les étudiant.e.s trouvaient la notion d'habitus «déterministe", en raison de la liberté de choix qu'ils croyaient avoir pour leur 
propre vie, une vie qui ne leur apparaissait pas tant contrainte ni par leurs conditions de naissance ni d'éducation au sein d'un certain milieu social. Autour de la notion d'habitus et de reproduction sociale, les réactions donnèrent également lieu à des échanges passionnés pour montrer comment leurs propres histoires de vie montraient aussi leur émancipation, voire leur évolution vers le haut de l'échelle sociale, par rapport à leurs parents qui n'avaient pas fait d'études, par exemple. Cependant, les étudiant·es sont globalement fasciné.e.s par ces notions, qui leur permettent une nouvelle lecture du monde social, tant à titre personnel - avec une prise de conscience du poids de leur famille et de leur éducation - qu'à titre professionnel, par rapport notamment à la violence symbolique potentielle que le travail social peut exercer sur des publics vulnérables, contraints, par exemple, de devoir demander une aide financière soumise à un examen approfondi de leurs conditions de vie et de leur revenu ainsi qu'à un contrôle serré des actions menées pour «sortir» au plus vite de l'assistance publique.

Sur la notion de culture et en réaction à diverses études de cas, les étudiant.e.s étaient partagé.e.s. D'une part, ils avaient beaucoup de peine à admettre que les «cultures» n'étaient pas des blocs homogènes déterminés par des traits recensables un à un, et les exemples affluaient pour montrer les différences entre cultures de différentes sociétés marquées par des traits nationaux ou religieux. Si les étudiant.e.s pouvaient entrevoir et comprendre les exemples tirés de sociétés autres (non occidentales), les concernant de loin, ils peinaient à rapporter leurs raisonnements à des territoires proches, comme si les personnes de nationalité suisse étaient toutes différentes et que l'on ne pouvait pas parler d'elles globalement, à la différence des «Africain.e.s». Ce n'est que quand je proposai de considérer ce que les Genevois.e.s disaient des Vaudois.e.s ou des Valaisan.ne.s et vice-versa que les stéréotypes furent exprimés et purent être replacés en situation. Par ailleurs, les exemples liés à la naturalisation, travaillés dans plusieurs de mes recherches, permirent de nombreux échanges sur la diversité des références et firent écho au sentiment d'appartenance que maint·e·s étudiant·es expérimentaient - étant eux-mêmes en train de passer ou ayant passé par des procédures de naturalisation - se sentant, par exemple, à la fois de «leur» quartier ou de «leur» commune en Suisse et d'origine nationale italienne, espagnole, portugaise ou kosovare. De même, la recherche sur les «couples mixtes» (Ossipow \& Waldis, 2002) permettait d'interroger la «différence de culture», à l'intérieur des couples formés soit par des étudiant·e•s, soit par 
leurs parents. Des exemples liés à des travaux de Bachelor furent aussi pris en compte (et même présentés par leurs auteur.e.s comme témoignages à l'occasion d'un cours ex cathedra). Il fut notamment fait référence à l'expérience d'une jeune femme d'origine juive, travailleuse sociale, ayant épousé un jeune homme ouvrier, d'origine musulmane. Ce mariage, d'abord très mal considéré par la famille de la jeune femme, puisqu'il enfreignait deux règles non dites (celle de la différence de confession et celle de la différence de milieux sociaux), fut, petit à petit accepté. Mais la jeune femme avait aussi raconté une anecdote étonnante qui montrait les difficultés, voire les préjugés que doivent affronter les partenaires d'un couple binational: en train d'allaiter son premier enfant lors d'une visite à la synagogue, elle entendit une femme, non loin d'elle, s'interroger à haute voix sur le tableau formé par la mère allaitante (blanche) et son bébé (noir): «Tiens, je ne savais pas qu'on pouvait allaiter quand on avait adopté.» Ce propos surprenant avait aussi une connotation ethnicisante, voire racialisante ou carrément raciste qui n'échappa pas aux étudiant.e·s, qui se demandèrent comment on pouvait imaginer que le bébé allaité pouvait ne pas être celui de sa mère, puisque noir. ${ }^{7}$

Le danger de coller une étiquette en fonction du faciès ou de ramener indéfiniment les personnes à leurs origines, quand elles ne le réclament pas ou qu'elles ne veulent pas en faire stratégiquement usage, fut expliqué de diverses manières, y compris par le témoignage direct d'étudiant.e.s «de couleur" présent.e.s dans la salle. Mais c'est probablement la vidéo Where are you from ${ }^{8}$ qui eut le plus d'effet pédagogique, certain.e.s étudiant.e.s la diffusant à leur tour. Cette vidéo relate un dialogue entre une joggeuse et un joggeur se rencontrant dans un parc aux Etats-Unis. Le premier, blanc et blond, demande à la seconde d'où elle vient en raison de son air «asiatique». Malgré sa très claire réponse, qui réfère par deux fois au fait qu'elle vient de San Francisco, le jeune homme cherche à lui faire énoncer une autre origine, qu'il définit finalement comme coréenne. La jeune femme lui demande alors à son tour d'où il vient, une question qu'il ne semble pas comprendre puisqu'il ne se voit que comme Américain. La jeune femme s'amuse toutefois à imiter une danse anglaise pour renvoyer

7 On notera toutefois que cela pourrait être possible, car la mère pourrait allaiter un autre bébé en sus du sien.

8 Consulté le I8.04.20I8 sur https://www.youtube.com/watch?v=crAv5ttax2I 
aux migrations venues d'Angleterre dès le $I 7^{\mathrm{e}}$ siècle, montrant ainsi l'absurdité qu'il y a à vouloir remonter aux origines en toutes circonstances, surtout lorsque les protagonistes n'y tiennent pas.

La question des rites, qui est peut-être celle qui intéresse le plus les étudiant.e.s et les professionnel-le·s du travail social, pensant que les «rites ont disparu dans nos sociétés occidentales» et donc soucieuses et soucieux d'en (ré)injecter dans leurs pratiques, surprit l'assemblée à plusieurs reprises. Le public du cours semblait aussi satisfait de voir que le travail social était facile à ritualiser (s'il ne l'était pas déjà). Alors que nous étions encore en cours de recherche sur le placement juvénile (Ossipow, Berthod \& Aeby, 20I4), les étudiant.e.s furent invité.e.s à analyser certaines de nos données sur les rites en foyer d'éducation. Analysant le récit d'une fête d'anniversaire durant laquelle les membres de l'équipe éducative et les résident.e.s offrent des cadeaux aux adolescents placés en foyer (il s'agissait ici d'un foyer de garçons), un étudiant fut choqué de remarquer qu'un éducateur avait offert à son «référé» (le jeune dont il est plus particulièrement responsable) une «banane» (un sac qui se porte autour de la taille) pour remplacer celle qu'il avait perdue lors d'une fugue. Cela lui paraissait incompatible avec la position du référent et avec ce qu'il avait déjà compris du travail éducatif sur la base de ses stages et de ses cours. A son avis, offrir un objet perdu lors d'une fugue revenait à la cautionner et à accepter une forme de rébellion. Si la réunion de foyer envisagée comme un rite de régulation de la vie quotidienne dans une institution de placement fut immédiatement comprise dans toutes ses dimensions de normalisation, celle de la fête de Noël, célébrée de façon laïque dans un des foyers (toujours le foyer de garçons), en étonna plus d'un.e, surtout quand les étudiant.e.s s'aperçurent que, durant le repas, c'étaient les éducatrices et les éducateurs qui servaient les adolescent.e•s placé.e·s (Ossipow, 2014). Le recours à la théorie de Victor Turner (I990), qui explique que certaines phases du rite (celle de liminalité, entre deux moments ou statuts) permettent de vivre les choses «à l'envers » ou d'inverser les hiérarchies, pour critiquer des dimensions de pouvoir, pour permettre de tisser des liens différents et inhabituels l'espace d'un moment, pour «retourner» des situations, sembla alors validé. Les étudiant.e.s furent étonné.e.s du fait que des équipes éducatives semblaient avoir inventé un bout de rituel sans apparemment connaître les théories des rites.

En somme, les réactions des étudiant·e·s furent diverses et variées, certain.e.s déplorant le cadre trop théorique, et donc compliqué, des concepts 
à comprendre, mais faisant contre mauvaise fortune bon cœur puisqu'ils pouvaient aussi tisser des liens avec leurs expériences personnelles du travail social. Si je fais le bilan de ce cours (sans savoir autrement que par les corrections d'examens si les notions avaient été réellement comprises par toutes et tous et utilisées dans d'autres exercices pratiques ou travaux par la suite), je constate que, bien que fondés sur des concepts de base développés par des anthropologues «classiques» n'ayant pas enquêté dans le champ du travail social, ces outils permettent néanmoins d'analyser ledit travail social et de s'interroger en retour sur ses propres pratiques. Décentration et réflexivité pouvaient donc faire bon ménage, même si la transmission (à l'exception des exemples personnels livrés en cours et des analyses menées en séminaires) était par trop unidirectionnelle (de l'enseignant.e à l'étudiant.e). Toutefois, les analyses des étudiant.e.s sur certains matériaux de recherches ont été très utiles aux chercheur·e.s: les étudiant.e.s constituent en effet un public extrêmement observateur et critique, détectant parfois immédiatement ce qui ne fonctionne pas dans la démonstration et forçant - par conséquent - les enseignant.e.s chercheur.e.s à réviser leur copie.

\section{Conclusion}

Ce module et l'enseignement spécifique d'anthropologie et sociologie qui y prenait place, dont l'objectif était d'étudier comment des personnes font société - à la fois par les alliances qu'elles créent et par les relations hiérarchisées et ritualisées qui organisent leur vie -, présente à mon avis un bon exemple d'articulation entre transmission de théories générales, souvent disciplinaires, et résultats spécifiques de recherche. La question de l'échange et de la réciprocité pourrait, par exemple, inciter les futur.e.s professionnel-le.s à renouveler leur regard sur la thématique du lien social, une notion beaucoup utilisée par les étudiant·e·s et les professionnel-le·s, mais peu sujette à une intense réflexivité, comme si l'effritement du lien social était d'emblée une donnée avérée qui ne prêtait plus à discussion et la cohésion sociale (une notion qui lui est liée), un idéal à maintenir. Comme on l'a vu, la thématique de la distinction replace le travail social au cœur des inégalités de classe, dont les étudiants·e.s pourraient davantage tenir compte pour éviter de considérer en premier lieu les usagères et usagers de divers dispositifs comme affaiblis ou vulnérables, du fait 
des ruptures personnelles ou familiales rencontrées dans leurs parcours de vie. ${ }^{9}$ Les développements liés à la notion de culture ont pour avantage de faire réfléchir les futur.e.s travailleuses et travailleurs sociaux sur des notions souvent peu déconstruites. Ces développements devraient d'ailleurs être poursuivis et débattus au fil de l'apparition de nouvelles notions dans le lexique du travail social, dans les discours des politiques et dans les recherches en sciences sociales. On constate, par exemple, que le terme de culture a peut-être évincé celui de race ou d'ethnie (voir supra), mais qu'il tend lui-même à être remplacé par celui de "communauté», dont les frontières et l'homogénéité ne sont, pour l'instant, que rarement questionnées. La thématique des rites, quant à elle, offre un angle d'approche qui, si elle ne se suffit pas à elle-même, peut déboucher sur l'analyse du fonctionnement des institutions. Elle permet de retravailler certains dispositifs en modifiant les rituels observés en divers lieux du travail social, qui cachent autant qu'ils révèlent les intentions et objectifs des professionnel.le·s (échanger, réguler, favoriser des apprentissages, laisser s'exprimer des tensions, proposer des renversements temporaires des hiérarchies).

Si l'on reprend maintenant la question de la présence de la recherche dans l'enseignement abordée en introduction, il est intéressant de faire référence à un débat ayant émergé en France, notamment après que des journalistes de Libération ${ }^{10}$ ont relaté l'avis de deux étudiant.e.s qui pensaient que l'enseignement de niveau bachelor devrait être donné uniquement par des enseignant·e.s non chercheur.e.s, comme c'est en général le cas au niveau du secondaire, donc en séparant clairement l'enseignement de la recherche. Les étudiant.e.s justifiaient leur demande en expliquant que les enseignant.e.s chercheur.e.s consacraient trop de temps à leur recherche et pas assez à leur cours ou à leur pédagogie. Ils faisaient évidemment l'impasse, soulignent les journalistes, sur le fait que ces chercheur·e.s étaient obligé.e.s d'agir ainsi pour faire face à la concurrence acharnée qui s'installait entre eux en vue d'obtenir un poste académique (le nombre d'articles publiés, le nombre de citations dont on fait l'objet, la hauteur des fonds décrochés étant considérés pour l'obtention d'un tel poste, et non les enseignements délivrés).

Le fait que la HES-SO soit obligée de considérer la recherche comme une de ses missions pour être considérée comme une haute école est une chance! Pouvoir susciter la réflexion et la réflexivité à partir d'un programme (multi)disciplinaire qui ne poursuit pas d'impératif utilitaire 
immédiat est également d'une grande opportunité, même si les chercheur.e.s doivent effectivement avoir du temps pour articuler au mieux recherche et enseignement. Mais c'est surtout aux étudiant.e.s qu'il faudrait laisser du temps au lieu de leur imposer un horaire digne du secondaire (ce qu'on appelle notamment à Genève le cycle d'orientation). Il faudrait qu'eux-mêmes puissent se nourrir des enseignements reçus en lisant d'autres textes, en suivant d'autres cours «qui ne servent à rien directement", comme les conférences organisées par les Midis de la recherche, ${ }^{11}$ permettant de débattre dans d'autres arènes que celles des cours, en présence de professionnel.le.s du travail social ou de membres du grand public. On pourrait aussi imaginer que les étudiant.e.s choisissent de tenir un journal de bord lié à certains cours et que ce journal, une fois retranscrit et synthétisé, puisse faire l'objet d'un bout de validation ou encore, comme c'est le cas dans le module autour de la pensée critique, qu'ils puissent rédiger des textes sur leur expérience, qui seraient ensuite publiés sans censure dans le cadre d'un essai lié à certaines thématiques philosophiques (Junod, Romagnoli \& Denervaud, 20I7). En outre, faire participer activement des étudiant.e.s à des activités de recherche, même pendant leur bachelor (c'est déjà le cas en master) pourrait aussi, même si l'accompagnement risque d'être chronophage et parfois dangereux, ${ }^{12}$ apparaître comme extrêmement fructueux, ne serait-ce que parce que cela habitue les futur.e.s professionnel-le.s à saisir l'utilité de la recherche autant fondamentale qu'immédiatement appliquée, une utilité à laquelle ils pourront être sensibles au moment de leur entrée sur le terrain professionnel. Pour ce faire, il faut évidemment que le nouveau Programme d'enseignement cadre (PEC), en train d'être établi, laisse de la vacance et de l'espace aux étudiant.e.s pour encore favoriser davantage leur réflexivité.

9 Sur ce point, voir, par exemple, le mandat de recherche effectué pour l'Hospice général (Mabillard, Ossipow, Delfortrie \& Peradotto, 2016).

12 Suivre le lien suivant: http://www.liberation.fr/societe/2015/06/29/recherche-etenseignement-freres-ennemis_I339593

Voir, par exemple, celui qu'Eva Nada et moi-même avons organisé en 2016-2017, sur https:/www.hesge.ch/hets/actualites/20I6/midis-recherche-20I6-20I7-precarites 


\section{Références bibliographiques}

Abélès, M. (2007). Le Spectacle du pouvoir. Paris: L'Herne.

Barth, F. (I995 [I969]). Les groupes ethniques et leurs frontières. Dans P. Poutignat \& J. Streiff-Fenart, Théories de l'ethnicité (pp. 203-249). Paris: Presses Universitaires de France.

Berger, P. \& Luckmann, T. (I966). La construction sociale de la réalité. Paris: Klincksieck.

Bourdieu, P. (I982). Les rites comme actes d'institution. Actes de la recherche en sciences sociales, 43(I), 58-63.

Bourdieu, P. (I986). La Distinction. Critique sociale du jugement. Paris: Minuit.

Bozon, M. (2002). Des rites de passage aux «premières fois». Une expérimentation sans fins. Agora 28(I), 22-33.

Collard, C. (2000). Femmes échangées, femmes échangistes. L’Homme, I 54-I55, IOI-II6.

Cuche, D. (I996). La notion de culture dans les sciences sociales. Paris: La Découverte.

Durkheim, E. (2003 [I9I2]). Les formes élémentaires de la vie religieuse. Paris: Presses Universitaires de France.

Eckmann, M. \& Fleury, M. (2005). Racisme et citoyenneté. Un outil pour la réflexion et l'action. Genève: Editions ies.

Eckmann, M., Sebeledi, D., Bouhadouza, V. \& Wicht, L. (2009). L'incident raciste au quotidien: représentations, dilemmes et interventions des travailleurs sociaux et des enseignants. Genève: Editions ies.

Ernaux, A. (1983). La Place. Paris: Gallimard.

Fraser, N. (2005). Qu'est-ce que la justice sociale? Reconnaissance et redistribution. Paris: La Découverte.

Gennep, A. van ([I909] I98I). Les rites de passage: étude systématique des rites... Paris: Editions Picard.

Guillaumin, C. (2005). "Je sais bien mais quand même», ou les avatars de la notion de race. Dans M. Eckmann \& M. Fleury, Racisme et citoyenneté. Un outil pour la réflexion et l'action (pp. I9-24). Genève: Editions ies.

Hénaff, M. (2002) Le prix de la vérité. Le don, l'argent et la philosophie. Paris: Seuil. Honneth, A. (2000). La lutte pour la reconnaissance. Paris: Le Cerf.

Jeudy-Ballini, M. (2004). L'Art des échanges. Penser le lien social chez les Sulka (PapouasieNouvelle-Guinée). Lausanne: Payot.

Junod, R., Romagnoli, S. \& Denervaud, J.-M. (20I7). L'inquiétude pour le monde et la pensée critique. Genève: Editions ies.

Lahire, B. (1998). L’homme pluriel. Les ressorts de l'action. Paris: Nathan.

Lavanchy, A., Gajardo, A. \& Dervin, F. (20I I). Anthropologies de l’interculturalité. Paris: L'Harmattan.

Lévi-Strauss, C. (I952). Le Père Noël supplicié. Les Temps modernes, 77, I572-I590.

Lévi-Strauss, C. (I967 [I949]). Les structures élémentaires de la parenté. Paris: Mouton.

Lévi-Strauss, C. (200I [I950]). Introduction à l'œuvre de Marcel Mauss. Dans M. Mauss, Sociologie et anthropologie (pp. IX-LII). Paris: Presses Universitaires de France. 
I29 | La recherche anthropologique dans l'enseignement en travail social

Mabillard, J., Ossipow, L., Delfortrie, J. \& Peradotto, J. (20I6). Profils et motifs des nouvelles entrées à l'aide sociale 20I5. [Rapport d'étude - I5 juillet 20I6; non publié]. Genève: Haute école de travail social.

Mauss, M. (200I [I950]). Essai sur le don. Forme et raison de l'échange dans les sociétés archaïques. Dans M. Mauss, Sociologie et anthropologie (pp. I45-279). Paris: Presses Universitaires de France.

Malinowski, B. (I963[I922]). Les Argonautes du Pacifique occidental. Paris: Gallimard.

Mauzé, M. (I986). Boas, les Kwagul et le potlatch. Eléments pour une réévaluation, suivi des commentaires de C. Meillassoux, A. Testart, D. Legros, S. Gruzinski, et d'une réponse de M. Mauzé. L’Homme, 26(I00), 2 I-63. Consulté le I I.04.20I8 sur HAL Id: halshs-007 I4450 https://halshs.archives-ouvertes.fr/halshs-007 I 4450

Ossipow, L. (I997). La cuisine du corps et de l'âme: approche ethnologique du végétarisme, du végétalisme, du crudivorisme et de la macrobiotique en Suisse. Neuchâtel/Paris: Institut d'ethnologie/Maison des sciences de l'homme.

Ossipow, L. (20I4). Se nourrir en foyer d'éducation: entre nécessité éducative et plaisirs commensaux. Sociologie et sociétés, XLVI(2), 205-228.

Ossipow, L., Berthod, M.-A. \& Aeby, G. (20I4). Les miroirs de l'adolescence. Anthropologie du placement juvénile. Lausanne: Antipodes.

Ossipow, L., Lambelet, A. \& Csupor, I. (2008). De l'aide à la reconnaissance. Ethnographie de l'action sociale. Genève: Editions ies.

Ossipow, L. \& Waldis, B. (2002). Terrains croisés: réseaux et couples turco-suisses à Berne et à Neuchâtel. Ethnologie française, XXXII(2), 283-294.

Poutignat, P. \& Streiff-Fenart, J. (I995). Théories de l'ethnicité. Paris: Presses Universitaires de France.

Rivière, C. (1995). Les rites profanes: Paris: Presses Universitaires de France.

Rivière, C. (1998). Les liturgies politiques. Paris: Presses Universitaires de France.

Sahlins, M. (I976). Age de pierre, âge d'abondance: l'économie des sociétés primitives. Paris: Gallimard.

Testart, A. (200I). Echange marchand, échange non marchand. Revue française de sociologie, 42 (4), 7 I9-748.

Turner, V. (I990 [I969]). Le phénomène rituel. Structure et contre-structure. Paris: Presses Universitaires de France. 



\title{
La posture professionnelle et la réflexivité en travail social envisagées sous l'angle ethnographique et esthétique
}

\author{
Francis Loser
}

\section{Introduction}

Cette contribution se propose d'interroger la posture professionnelle en travail social, notion souvent invoquée par les praticien.ne.s pour expliquer les valeurs qui inspirent leur action, légitimer le cap donné à leurs interventions ou défendre leurs prises de décision. Censés justifier une action ou un comportement, les mécanismes à l'œuvre dans la posture professionnelle sont peu ou prou explicités dans la littérature professionnelle. Le plus souvent, la posture est ramenée à la personnalité du ou de la praticien.ne - «le principal outil de l'éducateur, c'est sa personnalité» (Capul \& Lemay, 2005: I22) - et réduite à la dimension psychologique, aux mécanismes psychoaffectifs (transfert et contre-transfert, résonances, etc.). Pourtant, le terme de posture renvoie au corps - «Attitude particulière du corps", "Position du corps ou d'une de ses parties dans l'espace» et à un positionnement - «Situation d'une personne. Se trouver en bonne ou en mauvaise posture. Synonymes: condition, situation, position" (Le Petit Robert, I990).

La notion de posture professionnelle implique ainsi de considérer le rapport au corps, la manière de se tenir, mais aussi la position, qui peut à la fois être entendue comme l'affirmation d'une préférence (valeur, morale, etc.), d'un rôle social choisi (autodétermination) et d'une assignation de place (position sociale, rang dans une hiérarchie, etc.). La notion de posture professionnelle ouvre sur des perspectives à la fois psychologique, sociale, éthique et esthétique extrêmement propices pour penser la part dynamique de l'intervention professionnelle conçue en termes d'agir situé.

Cette question est, à mon sens, insuffisamment explorée dans les réflexions professionnelles et il m'a paru intéressant de l'examiner de plus près en opérant un détour par la recherche et, plus précisément, par les réflexions issues de l'observation participante. Outre le fait qu'elle 
autorise un recueil d'informations relatif aux actrices et acteurs observés et à leurs pratiques, la méthode ethnographique oblige les chercheur.e.s à penser leur implication dans le terrain, pas uniquement sur un mode rhétorique, mais «en attention gardée présente à ses propres fonctionnements " (Canter Kohn \& Nègre, 2003: 193), ces derniers participant pleinement à la construction des données de la recherche.

Comme nous le verrons ci-après, les praticien·ne.s en travail social pourraient tirer profit de cette méthode de recherche pour les aider à préciser ce que recouvrent les notions de posture et de réflexivité, qu'il s'agisse de repérer leur positionnement ou de décoder les déterminants sociaux qui traversent les situations dans lesquelles elles et ils sont engagés.

L'article est construit en plusieurs parties et débutera par un examen approfondi de la notion de posture professionnelle afin d'en appréhender les multiples contours. Dans un second temps, le questionnement sera élargi par des apports portant sur la méthodologie relative aux enquêtes qui impliquent une immersion sur le terrain. Le focus sera placé sur le journal de terrain, texte dans lequel vient se cristalliser la question du rapport objet-sujet. Enfin, en rapport à la formation de base et continue en travail social, la posture requise dans l'intervention sociale sera considérée à partir de la posture de recherche.

\section{Posture et pratiques professionnelles}

Ainsi que le notent Bertrand Ravon et Jacques Ion, le travail social ne se situe plus aujourd'hui dans l'idéal de réparation, mais dans une logique d'intervention qui repose sur « un travail relationnel de présence et de soutien à autrui (pratiques du care) [...]» (20I 2: 6). La place centrale qu'occupe la composante relationnelle dans l'activité des professionnel.le.s du social est également soulignée par Michel Autès, qui estime que «la spécificité du travail social est d'être une relation de service où les services sont au service de la relation" (2013: 48). A ce sujet, il importe de relever que «le travail émotionnel est une dimension ordinaire de l'activité de travail mais celleci occupe une place plus importante dans les emplois de service» (Avril, Cartier \& Serre, 2010: 95). Cela a amené Stéphanie Boujut à s'intéresser à la gestion de la position professionnelle en tant que modalité pratique de l'exercice professionnel. Pour la sociologue, la modalité de cette gestion «implique de mettre en relief les moments où cette gestion s'opère dans 
l'interaction. Ces moments sont le plus souvent émotionnels» (2005: I43). Et l'auteure de remarquer que «le registre émotionnel [...] est un élément central de la compréhension des pratiques des travailleurs sociaux dans leurs relations au public» (2005: I43-I44). En tant que relation de service, le travail social exige donc des praticien.ne.s qu'elles et ils développent la gestion des émotions afin «d'inscrire la demande d'aide dans un contexte professionnel et non pas émotionnel» (2005: I49).

La gestion des émotions fait partie intégrante du savoir pratique en travail social et, pour leur permettre d'être à l'aise dans la plupart des situations de face-à-face avec les bénéficiaires, les praticien.ne·s développent des stratégies pour éviter d'être envahi.e.s par la vulnérabilité de l'autre. Lors d'une enquête portant sur les interactions entre bénéficiaires et professionnel-le.s de divers domaines d'activité (infirmières et infirmiers, agent.e.s de l'ANPE ${ }^{1}$ ou des caisses d'allocations familiales), Philippe Corcuff a mis en évidence qu'il s'agit là d'un «régime d'interpellation éthique dans le face à face, mode de rapport ordinaire à l'action qui peut se définir comme «le fait d'être ‘pris`, en pratique et de manière non nécessairement réfléchie, par un sentiment de responsabilité vis-à-vis de la détresse d'autrui, dans le face à face et la proximité des corps " (I998: 5). A partir de l'amour dé-mesuré ou agapé modélisé par Luc Boltanski (I990), le chercheur souligne que la reconnaissance de la détresse de l'autre peut entraîner chez le ou la professionnel-le un au-delà de la mesure, qui peut s'exprimer autant par la compassion que par la violence liée à la présence de l'autre souffrant.e qui menace sa tranquillité. Ce phénomène est clairement visible chez les infirmières et infirmiers qui, confrontés à une proximité des corps, se construisent un système de «blindage" pour pallier l'exposition continue à la souffrance (Mercadier, 2006; Molinier, 2013).

Dans le cadre d'entretiens menés avec des éducatrices et éducateurs qui accompagnent des adolescent.e.s (Loser, 2013), l'interpellation par l'autre était au cœur des réflexions car, comme le précisait un praticien: "On vit avec ces jeunes on partage l'intimité de ces jeunes on vit leur quotidien.» Une de ses collègues, à laquelle je demandai si l'activité éducative pouvait être assimilée à toute autre activité professionnelle, éclata de rire avant de préciser que, pour elle, «c'est nettement plus! Il y a un investissement de part et d'autre, ça déborde forcément». Cette proximité en excès vient

1 Ancien Pôle emploi, équivalent des offices cantonaux de l'emploi. 
fragiliser les frontières entre les registres professionnel et personnel. Lors de mes temps d'immersion sur le terrain, il m'a été donné d'observer des scènes durant lesquelles les débordements n'étaient pas que de nature émotionnelle. Un jour, par exemple, j'ai vu un jeune passer subrepticement derrière un éducateur pour, d'un coup, le ceinturer et le soulever de terre, ce qui tend à faire penser que les interactions entre praticien.ne.s et bénéficiaires ne sont pas sans risque.

Lors des interactions de face-à-face en travail social, le positionnement professionnel, qui repose sur une asymétrie des rapports et une discrimination positive (Boujut, 2005), se trouve parfois bousculé par l'ambiguïté des situations (proximité qui invite à une confusion des rôles, par exemple) et le comportement des bénéficiaires (agressivité verbale et parfois agie, demandes déplacées, etc.). Pour le coup, la posture professionnelle occupe une place cruciale dans l'intervention sociale, qui implique un engagement conséquent des praticien·ne·s qui risquent "d'être pris" (Corcuff, I998) dans les situations d'interaction et d'être assignés à une place en plus d' «être affectés» (Favret-Saada, I990).

La nature de l'engagement professionnel en travail social met en perspective la centralité du corps en termes d'agir - le je peux (Henry, 2006) -, dimension fréquemment laissée dans l'ombre. Hans Joas (2008) note que dans les théories de l'action, le plus souvent, il est implicitement question d'un agir rationnel. Outre un caractère téléologique de l'action, ce dernier se signale par une autonomie de l'actrice ou de l'acteur, conçu•e hors de tout contexte et en pleine capacité de maîtriser son corps. Or, il n'en est rien et, afin de mieux saisir les mécanismes qui interviennent dans la posture professionnelle, il convient de s'intéresser au vif de l'action et à la composante corporelle de l'agir humain.

Si le corps est peu présent en travail social, ce phénomène mérite d'être rapporté à la place généralement accordée au corps dans le monde occidental. Selon David Le Breton (I990; 2013), du fait d'une tradition philosophique fortement imprégnée par le platonisme, l'Occident a de tout temps nourri un soupçon à l'égard du corps et un dualisme qui salue les vertus de l'esprit, de l'âme, de la connaissance et du Bien en opposition au corps entrevu comme le lieu de la captivité, de la mort, de l'ignorance et des plaisirs troubles. L'anthropologue Edward T. Hall s'étonne, lui aussi, de l'ancrage culturel occidental, pétri de dualité, qui «détourne notre attention de la causalité multiple» (I984: I58) et classe pratiquement tout par catégories exclusives. 


\section{L'agir professionnel et sa dimension corporelle}

En dépit d'une pensée dualiste omniprésente, les contributions d'auteur.e.s qui s'intéressent au corps, à sa réalité ontologique (Merleau-Ponty, I945; Henry, 2006), à ses multiples fonctions (Mauss, 200I ; Joas, 2008; Winkin, 200I; Sennett, 20Io, Hall, I984) et à son rôle normatif (Goffman, I973, I974; Joas, 2008; Kaufmann, I995, I997; Lahire, 200I ; Detrez, 2002; etc.) ne manquent pas.

Pierre Bourdieu (I980) estime pour sa part que le «sens pratique» demande à être rapporté à un "savoir par corps", alors que Gunter Gebauer et Christoph Wulf (2004) avancent que le corps opère comme une médiation entre la personne et son environnement du fait des apprentissages réalisés en famille, à l'école, au travail, etc. Le mimétisme constitue ainsi le socle des apprentissages sociaux par lesquels s'incorporent littéralement les codes et autres normes dans nos habitudes et gestes quotidiens. «Un individu ne peut pas faire autrement que d'être là en chair et en os ", nous rappelle Erving Goffman (1974: I36). Et, du fait de notre réalité incarnée, notre corps fonde la modalité première de notre rapport au monde - «J'ai un corps et je suis un corps" (Shusterman, 2007) -, le lieu frontière entre soi et les autres, l'intime et le social (Le Breton, 2007).

L'activité déployée en travail social et les habiletés pratiques qu'elle mobilise en situation renvoie à un ensemble de savoirs tacites, qu'il s'agisse du savoir pratique et de l'habitus (Bourdieu, I980), de la mètis (Clot, I998), des savoirs incorporés (Schön, I994), de la subjectivité de l'agir (Dejours, 2003) ou d'une présence (Molinier, 2013). Le savoir pratique est revendiqué par les praticien.ne.s, qui disent agir par instinct, par intuition, hissant le feeling et l'intelligence pratique au rang d'une expertise qui, selon Matthew Crawford (20I0), ne manquerait pas de noblesse.

Etroitement lié au terrain, à la fois lieu d'apprentissage et d'exercice professionnel, le savoir pratique «est dans nos actes» (Schön, I994: 76), et se traduit le plus souvent en termes de "tour de main" et "arts de faire» (Certeau, I990). Il serait ici question d'«une intelligence du sujet, mal définie sinon par des neutres (avoir du flair, du tact, du goût, du jugement, de l'instinct, etc.) qui oscillent entre les régimes de l'esthétique, du cognitif ou du réflexe, comme si le ‘savoir-faire) se réduisait à un principe insaisissable du savoir" (Certeau, I990: I I2-I I3). 
A l'évidence, l'activité professionnelle est complexe et présente un caractère insaisissable car «l'action est opaque à celui-là même qui l'accomplit» (Vermersch, I994: 32). C'est ce qui a amené Vermersch à imaginer un dispositif sous la forme d'un entretien d'explicitation (I994) qui stimule la mémoire des praticien.ne.s en s'intéressant plus particulièrement aux émotions et sensations, donc au corps en tant que lieu des ajustements automatiques. Pour les mêmes raisons, Yves Clot (I998) a conçu des analyses de pratique qui autorisent les praticien.ne.s à accéder aux ressorts de l'action par le biais du visionnement de vidéos prises en cours d'activité.

Interroger la posture professionnelle à partir de la corporéité de l'agir offre un angle d'approche doublement heuristique puisqu'il permet, d'une part, d'ancrer la réflexion sur une base concrète, largement documentée et, d'autre part, de considérer l'activité sociale de manière élargie en y intégrant des aspects occultés ou peu pris en considération, notamment le poids des représentations sociales, la force des habitudes, l'affectivité ou encore la pré-réflexivité inhérente à l'agir.

Comme vu précédemment, l'agir humain oscille entre les régimes du cognitif, du réflexe et de l'esthétique entendue en tant qu'appréhension non dualiste du monde, qu'il s'agisse de soi et des autres ou de la raison et des émotions. Hans Joas attire notre attention sur le fait que, dans une théorie de l'action qui tente de surmonter une pensée dualiste, il s'agit d' «inclure aussi la passivité, la sensibilité, la réceptivité, la sérénité » (Joas, 200I: 35) au risque d'assimiler l'action à une simple implication activiste et d'en exclure sa part de corporéité. Le neurologue Antonio Damasio s'inscrit un peu dans la même veine lorsqu'il souligne que «nos pensées les plus élevées et nos actes les meilleurs, nos plus grandes joies et nos plus profondes peines, ont notre corps pour aune» (200I : I4).

Si une scission est observée entre le corps et l'esprit, ou la raison et les émotions, elle est également présente dans la distinction entre soi et les autres (Winnicott, I975; Mead, 2006), ce qui ne serait qu'une illusion résultant d'un long processus d'individuation depuis l'état non différencié du nourrisson. Pourtant, c'est grâce à une auto-affection originaire (Henry, 2004), à une affectivité primordiale du sujet (Depraz, 2006) inhérente à la condition incarnée de l'être humain, via son schéma corporel, que nous pouvons avoir une perception globale des situations et nous situer dans l'intersubjectivité. Cela expliquerait le phénomène de "sympathie», qui est une forme d'expression du rapport social antérieure à toute intentionnalité consciente du sujet. 
Pour Vinciane Despret, nos émotions seraient autant de versions du monde et de manières de l'habiter qui n'existent pas en soi, mais dans la relation à autrui. Selon elle, les émotions sont des modes de définition et de régulation des relations sociales et du soi dans un ordre moral. C'est pourquoi «l'émotion s'y définit alors comme une manière par laquelle nous négocions notre rapport à nous-mêmes, au monde, et aux autres" (200I : 283), perspective qui coïncide avec l'idée d'un soi qui dépend des interactions sociales et, pour le coup, de sentiments et émotions conçues comme des faits sociaux. Par ailleurs, en rapport à la compassion éprouvée par les professionnel.le.s, Corcuff souligne que «cette sensibilité au singulier est souvent corporelle» (I998: 6), remarque qui rejoint les réflexions de Joas (2008).

Dans la mesure où le travail social est contingent et implique un savoir en actes, un engagement corporel et sensible, les praticien.ne.s sont appelé.e.s à négocier le rapport aux autres par le biais d'une attention à la composante émotionnelle des interactions qui n'est pas forcément conscientisée. Cette configuration particulière de l'activité, qui suppose «une part implicite dans sa réalisation, précisément pour celui qui l'effectue» (Vermersch, I994: I8), fait plus largement écho à la normativité de l'agir mise en relief dans les travaux sociologiques. Elle n'est pas sans soulever des interrogations concernant la posture professionnelle. Comment invoquer une expertise professionnelle fondée sur une base aussi insaisissable qu'une affectivité primordiale du sujet convertie en "savoir pratique», qui échappe à la conscience des praticien.ne·s? Pour tenter de répondre à cette question, je propose d'élargir la réflexion en m'intéressant à la posture de chercheur.e engagé.e dans un processus d'observation participante. Nous le verrons, cette méthode est propice à penser ce que signifie un agir incarné inhérent à une immersion sur le terrain, prisme aux multiples facettes qui, selon moi, concerne autant la recherche que l'intervention sociale.

\section{Observation participante et posture esthétique de la ou du chercheur.e}

Couplée à des entretiens, l'observation participante constitue une méthode ethnographique féconde pour appréhender un terrain de recherche et saisir l'ensemble des phénomènes qui s'y produisent (Loser, 2010; Loser \& 
Waldis, 20I7). Cette méthode exige néanmoins de clarifier certains points, telles les notions de posture professionnelle et de réflexivité qui forment le cœur de ma présente réflexion.

L'attention des chercheur.e.s immergé.e.s dans un terrain est à l'évidence un premier point crucial à examiner. L'observation in situ de scènes où interagissent entre eux des groupes de personnes exige de passer par une conscientisation du regard porté sur les phénomènes entrevus, en tant que ce dernier est constitutif de la construction des données. Olivier de Sardan note à ce propos que «les données sont la transformation en traces objectivées de morceaux de réels tels qu'ils ont été sélectionnés et perçus par le chercheur» (I995: 76). La posture de recherche implique, de fait, une distanciation du regard habitué (Canter Kohn \& Nègre, 2003), mais il n'en demeure pas moins que «les observations du chercheur sont structurées par ce qu'il cherche, par son langage, sa problématique, sa formation, sa personnalité. [...]» (Olivier de Sardan, I995: 76). En d'autres termes, Nadia Mohia relève que, sur le terrain, le ou la chercheur.e est une personne qui continue de vivre, qui «reste un sujet entier, engagé par une relation à l'autre totale, c'est-à-dire par sa subjectivité propre, son histoire personnelle et son appartenance à une société donnée» (Mohia, 2008: I8).

Ces réflexions croisées tendent à accréditer l'idée que l'observation participante relève d'une expérience humaine et subjective, une démarche expérienciée (Canter Kohn \& Nègre, 2003), ce qui implique, pour le ou la chercheur.e, de mener une "analyse réflexive sur les conditions dans lesquelles le processus de connaissance se développe sur le terrain» (Caratini, 2004: I8).

Afin de faire tenir le fragile équilibre entre implication et distanciation, composantes indissociables de la posture d'observation participante, le ou la scientifique est soumis·e à un effort constant de dédoublement, à un jeu d'alternance entre un être dedans et un être dehors. De fait, l'observation participante peut être assimilée à une forme de jeu car, comme le rappelle Caratini: “Jouer, c'est se dédoubler» (2004: 78).

Jean Rouch a très bien analysé cette manière de se dédoubler au cours d'une interview (2004). Lors du tournage de cérémonies d'exorcisme, le réalisateur et ethnologue disait appliquer une méthode - le ciné-transe - qui se signale par un œil «pris" par la charge émotionnelle de la scène filmée et l'autre œil, rivé sur le viseur de la caméra, qui observe la scène dans l'optique du résultat final visible à l'écran. Un peu à la façon du visionnement des rushes après le tournage, le ou la chercheur·e se remémore dans 
l'après-coup les scènes observées et tente de traduire ces morceaux de réels saisis in situ en traces objectivées dans son journal de terrain.

Cet exercice d'objectivation des données est délicat et demande aux chercheur.e.s de mener une opération en plusieurs temps. Tout d'abord, les temps d'immersion sur le terrain impliquent une confrontation à une multiplicité d'informations complexes, superposées les unes aux autres et imbriquées entre elles, que l'observation doit parvenir à saisir de manière approfondie, comme s'il s'agissait d' "essayer de lire (au sens de «construire une lecture de») un manuscrit étranger, défraîchi, plein d'ellipses, d'incohérences, de corrections suspectes [...]" (Clifford Geertz, I998: 80). Ensuite, les chercheur.e.s sont appelé.e.s à consigner dans leur journal de terrain l'ensemble des événements et interactions observés, et cela de la manière la plus détaillée possible, afin de produire ce que Geertz (I998) nomme une «description dense». Cette phase d'objectivation, qui est une mise à distance de l'expérience d'immersion, est essentielle et oblige le ou la chercheur.e à tenir compte du jeu de dédoublement mentionné plus haut en faisant preuve d'honnêteté. A ce sujet, James Clifford relève que si l'observatrice ou l'observateur accède à ce qui se passe à l'intérieur du terrain observé, son autorité expérientielle demande toutefois à être entrevue de manière critique, le j'y étais ne constitue pas un gage d'authenticité et de valeur absolue (2003: 274-275).

Dans la phase de mise en mots de l'expérience d'immersion, les émotions éprouvées sont des indicateurs connexes aux informations factuelles et autorisent bien souvent des analyses fécondes (Loser, 20I2). En rapport aux travaux d'Erving Goffman (I974; I99I), dans trois enquêtes de terrain, deux plus anciennes (Loser, 2010; 2013) et une plus récente (Loser \& Waldis, 2017), une partie des analyses ont été menées sur la base d'un examen attentif des situations d'embarras, qu'il s'agisse de l'embarras éprouvé en tant que chercheur.e ou de celui observé chez les personnes observées. Pour illustrer cela, je vais revenir sur des observations consignées dans le journal de terrain élaboré à l'occasion d'une investigation menée dans des ateliers d'expression et de création (Loser, 2010; 20I2). Alors que j'avais pour habitude de participer aux activités créatives par un travail de collage - images de magazines découpées et agencées sur une feuille de papier -, l'absence de journaux, lors d'un premier temps d'observation dans un atelier qui réunissait des personnes âgées, me contraignit à me rabattre sur des craies grasses. Aussitôt, des souvenirs de classe maternelle surgirent en moi, et cela de manière intense et prolongée. Une 
forme d'embarras me saisit alors, pour ne plus me quitter. Cette gêne trouva une première réponse lorsqu'un participant évoqua un souvenir d'enfance clairement attaché à sa scolarité en classe maternelle. L'embarras que j'avais ressenti dans ce terrain portait ainsi au-delà de contenus biographiques, hypothèse qui s'est confirmée au fil de mes observations dans le même atelier. Au cours de la même recherche, dans un atelier de modelage ouvert à tout public, une participante dans la cinquantaine arriva alcoolisée à l'une des séances. Ce jour-là, placée à la table de travail face à moi, elle n'eut de cesse de m'interpeller par des paroles et des gestes ambigus. De chercheur, j'étais d'un coup placé dans le rôle du mâle, et l'embarras me saisit durant tout le temps de l'atelier et dura par la suite encore. De fait, il m'a été difficile de rapporter cette scène dans mon journal de terrain tant elle faisait surgir en moi des sentiments contradictoires et conflictuels. Les réflexions de Michel Leiris - «Doit-on tout raconter? Doit-on choisir? Doit-on transfigurer? Je suis d'avis qu'il faut tout raconter» (Leiris, I98I : I87-I88) - m'ont aidé à en tirer parti et à admettre que, en tant que chercheur, j'étais non seulement observé, mais aussi assigné à une place qui pouvait s'avérer inconfortable. Ce constat m'a amené, une nouvelle fois, à vérifier l'importance des interactions sociales qui conditionnent le positionnement personnel, quand bien même ce dernier tente d'incarner l'affirmation de préférences attentives à la dimension éthique.

Dans le cadre d'une investigation dans des ateliers d'art visuel fréquentés par des artistes en situation de handicap, outre la question de l'artification des œuvres - processus par lequel les créations accèdent au rang d'œuvre d'art (Heinich \& Shapiro, 20I2) -, il importait de comprendre la manière dont les artistes passaient du statut dévalorisé de personne handicapée au statut valorisé d'artiste. Aussi, Barbara Waldis et moi-même nous sommes intéressés à saisir comment et quand s'opérait le jeu de bascule entre, respectivement, les situations de production de handicap et de production artistique (Loser \& Waldis, 20I7) en nous inspirant des travaux sur le Doing Gender de Candace West et Sarah Fenstermaker (I995). Comme lors de la précédente enquête mentionnée, les situations d'embarras ont constitué des indicateurs précieux pour l'analyse. Ce fut notamment le cas dans la scène suivante, qui se déroula dans un des ateliers d'art alors qu'une attachée culturelle s'entretenait avec un artiste afin de sélectionner des œuvres pour une exposition. Les questions posées étaient déjà en soi intéressantes à considérer, dans la mesure où elles portaient sur des aspects artistiques (les sources d'inspiration, le mode de procéder, etc.), entrecoupées par 
d'autres considérations plus ambiguës («Tu vis où? »; «Tu as une amie? »). L'attachée culturelle parlait-elle à un artiste ou à une personne «handicapée»? Cette hésitation mérite d'être rapportée aux travaux de Charles Gardou, qui note que les personnes en situation de handicap sont assignées à un état liminal, dans un entre-deux, «dans une zone où leur acceptation et leur reconnaissance restent équivoques» (Gardou, 2000: 75).

L'hésitation de l'attachée culturelle se mua apparemment en embarras lorsqu'elle demanda à l'artiste s'il désirait voir exposée une de ses œuvres favorites. A la question posée, l'artiste répondit simplement par un «Non » qui semble avoir surpris son interlocutrice, laquelle répliqua aussitôt par un «Oui, tu as tout à fait raison! » suivi d'un rire. Le rire est un acte non contrôlé (Gebauer \& Wulf, 2004) qui, dans l'interaction observée, est venu brouiller la communication. Le phénomène observé peut paraître anodin, mais notre enquête nous a montré qu'il n'était pas isolé et pouvait relever "de tout ce qui embarrasse spécifiquement les rencontres" (Goffman, I974: 96), et cela particulièrement dans les interactions entre personnes en situation de handicap et personnes «valides» (Murphy, I990), en raison des représentations sociales du handicap.

Durant un temps d'observation pendant lequel j'étais confronté à des personnes polyhandicapées, une forte gêne me saisit, alors que je suis habitué à côtoyer des personnes en situation de handicap. Cette situation d'embarras a été mise en mots dans mon journal de terrain (Loser, 2013): «Face à Katia et Anne, je me suis senti quelque peu déstabilisé. Ce sentiment est en partie dû à la difficulté de communication mais, je dois l'avouer, c'est surtout leur entrave corporelle qui me touche [...]» Etant clairement affecté lors de ce temps d'observation, mon embarras pouvait être mis sur le compte d'une confrontation à ma vulnérabilité, mais il relevait également d'une situation sociale qui engage une affectivité démesurée (Corcuff, I998).

Ce type d'interaction qui suscite l'embarras vient interroger la posture à adopter en tant que chercheur.e pour ne pas perdre la face et faire perdre celle de l'autre (Goffman, I973) ni perdre pied, afin de poursuivre les observations de terrain.

Dans la dernière illustration examinée, le chercheur que je suis s'est trouvé momentanément déstabilisé par une soudaine irruption d'affects inattendus. Il a fallu que j'en prenne conscience pour que je puisse à nouveau porter attention à la scène que je tentais de saisir. Cette «chimie» particulière a été étudiée par le neurologue Antonio Damasio, qui note que 
«l'organisme interagit avec l'environnement en tant que tout: l'interaction n'est pas le seul fait du corps, ni le seul fait du cerveau» (200I : I4). Contrairement à l'opinion courante, le chercheur précise que les perceptions d'émotions suivent «un agencement physiologique des plus curieux, puisque celuici a mis le cerveau dans l'obligation d'écouter le corps " (200I: I I3). De ce fait même, les "perceptions d'émotions ne sont ni fugitives ni insaisissables [et] ont une valeur cognitive, tout autant que les autres percepts» (200I: I3). Si l'on suit Damasio, la perception des émotions est essentielle car celles-ci participent aux rouages de la raison. Immergé dans le terrain, cette articulation entre émotions et raison m'a été rendue accessible grâce au recours à la double réduction phénoménologique husserlienne ou épochè, opération «par laquelle je mets entre parenthèses la thèse du monde et opère ce faisant une mise en suspens des préjugés et préconceptions" (Depraz, 2006: 204). Concrètement, l'épochè implique un geste qui vise un "devenir conscient", qui se déploie selon trois phases principales organiquement liées entre elles. Une première phase de suspension de l'attitude «naturelle», au profit d'une attention qui s'attache à une description phénoménologique des choses perçues, phase qu'il me semble possible de rattacher à la question: "Qu'est-ce qui se passe ici?" (Goffman, I99I); une seconde phase de conversion de l'attention, qui va se déporter de l'extérieur vers l'intérieur, sur les émotions éprouvées et, enfin, une troisième étape, où le sujet accueille l'expérience vécue et retourne vers la situation (Depraz, 2006: I04).

Comme l'évoque Jean-Claude Ameisen dans un dialogue avec Raphaël Enthoven (Enthoven et al., 2009: 29-50), porter attention aux situations d'embarras autorise à mettre en articulation deux façons complémentaires de saisir la réalité; ce qui est de l'ordre de la sensibilité - le sentir et ce qui participe de l'ordre de l'explication rationnelle - le comprendre. D'une certaine façon, cela rejoint une des réflexions de Sophie Caratini, qui estime que l'anthropologie demande aux chercheur.e.s de penser la différence et de s'y confronter en allant sur le terrain afin de l'expérimenter, quitte à «frôler la lisière entre la distinction et la confusion, de l'éprouver jusque dans leur chair» (2004: 4).

Plus largement, il semble intéressant d'établir une passerelle entre la double réduction phénoménologique décrite plus haut et l'attitude esthétique (Stolnitz, 2004), qui tranche du regard ordinaire qui capte les choses en fonction de leur intérêt utilitaire spécifique, de leur place dans l'ordre conceptuel du monde. Selon Richard Shusterman (2007), une perspective esthétique invite à s'arrêter sur la phénoménalité sensible de l'apparaître 
via l'éprouvé afin de dépasser notre dépendance à la vie irréfléchie et prendre conscience des filtres aux travers desquels nous appréhendons le monde (valeurs, position sociale, etc.).

\section{De l'activité préréflexive au «devenir conscient»}

A la lumière des éléments développés précédemment, il importe de revenir aux notions de posture et de réflexivité en rapport à la question de la conscience des praticien.ne.s en cours d'activité.

Autant une conception téléologique de l'agir implique un sujet autonome et conscient de son action afin de poursuivre les buts fixés, autant une détermination situationnelle de l'agir couperait le sujet de toute intention et conscience. Si l'on suit Hans Joas (2008), une voie médiane peut être envisagée si l'on conçoit un agir qui n'est pas coupé du contexte et qui ne réduit pas celui ou celle qui agit à un statut d'idiot sans discernement, selon la formule de Garfinkel (2007). Pour Joas, «le rapport à la situation et le rapport au but sont, dès le départ, interdépendants [...] car sans certaines dispositions finales, si vagues soient-elles, données ante actu sous la forme de besoins, d'intérêts et de normes, aucun événement ne pourrait présenter à nos yeux le caractère d'une situation, ce ne serait jamais qu'un fait dépourvu de signification" (Joas, 2008: I7I). Pour le sociologue, il paraît évident que le lien entre l'agir et la situation qui le suscite relève d'un registre préréflexif qui renvoie au corps en tant que siège de l'agir. Si le sens pratique, inhérent à la corporéité de l'agir, rime avec les habitudes et la routinisation de nos gestes, cela n'exclut toutefois pas l'intervention de la conscience, ne serait-ce que de manière épisodique, notamment quand l'activité engagée sur le plan préréflexif se voit empêchée. Pour sa part, Bernard Lahire (200I) estime que la rupture avec le sens pratique provoquée par l'ajustement réflexif, la planification ou le calcul n'a rien d'une situation de crise et apparaît dans les moments de vie ordinaire. La liste de commissions ou de vêtements à emporter dans sa valise et les itinéraires de trajets inhabituels illustrent cela. Les réflexions de Lahire interrogent la manière de concevoir le cadrage de l'action et, s'il reconnaît que «les acteurs sont souvent pris dans «le feu de l'action', il remarque que «ces derniers sont parfois aussi hors d'elle (pour la préparer ou se la remémorer, l'évaluer, en rediscuter, la narrer, la commenter, la théoriser )" (200I : 235). Cette observation est cruciale pour ne pas confiner l'activité des professionnel.le·s du 
social aux seuls temps d'interaction avec les bénéficiaires, pendant lesquels les modes opératoires préréflexifs jouent en plein. Ainsi, l'intervention sociale demande à être replacée dans un jeu de temporalité synchronique (Hall, I984) où l'instant des face-à-face s'articule à une durée plus longue, celle de l'accompagnement d'un.e bénéficiaire. Cette conception de l'activité englobe alors les temps de réflexion, de supervision, d'écriture, de réunion et d'échanges entre collègues, etc. Comme le précise Lahire, «si l'on ne réduit pas l'action à l'action de courte durée [...] alors on comprend que la réflexion, y compris la plus rationnelle, puisse intervenir dans le cours d'une action et même en constituer des temps ou des étapes obligées [...]» (200I : 272). De façon proche, Goffman relève qu'il y a la scène d'interaction en face-à-face, mais qu'il y aussi «ce qui se passe en coulisses, c'està-dire l'action avant ou après la scène, ou encore derrière elle, et qui est incompatible avec elle» (I99I: 2I5), manière de tenir compte du vécu des protagonistes. Pour Goffman, les interactions de face-à-face sont le lieu de jeux subtils car «ceux qui conversent doivent généralement faire preuve de tact, c'est-à-dire dissimuler des pensées ou des émotions qu'on sait par ailleurs réelles" (I99I : 2I7). Les stratégies constituent la base même des jeux, tels les échecs, où les feintes sont possibles dans le but de mettre l'adversaire sur une fausse piste. Il est assez improbable que, dans ces jeux de faceà-face, seul un mode opératoire préréflexif soit concerné, postulat auquel pourrait souscrire Richard Sennett lorsqu'il souligne que «tout bon artisan poursuit un dialogue entre pratiques concrètes et réflexion" (2010: 20).

Plus largement, ce débat autour de la conscience de la personne qui agit nous ramène à une critique de la conception dualiste du fonctionnement humain, pour laquelle la réflexion est forcément réduite à une dimension théorique et académique qui s'oppose au sens pratique (Lahire, 200I : 27), alors qu'il y a tout lieu de considérer un «faire-cognitif» (Certeau, I990). Plus largement, Sennett estime que «toutes les compétences, même les plus abstraites, sont au départ des pratiques physiques» (20I0: 2I).

Sur le terrain, les praticien·ne·s en travail social sont amené.e.s à devoir composer avec des situations non structurées (Schön, I994), qui contiennent une multiplicité d'informations complexes et superposées les unes aux autres et devraient, de ce fait, pouvoir tirer bénéfice des apports méthodologique relatifs à l'observation participante.

Comme relevé en début d'article, l'auto-observation est bien présente en travail social, mais elle se rapporte le plus souvent aux enjeux d'ordre psychoaffectif (transfert et contre-transfert, résonances, etc.) au détriment d'une 
lecture élargie qui tient compte des déterminants sociaux. Au détour d'une enquête menée auprès d'assistantes sociales qui exercent dans le milieu hospitalier, Jean-François Gaspar a repéré que la volonté de ces dernières de se rapprocher des bénéficiaires «s'accompagne d'une occultation, au moins partielle, de la distance sociale qui sépare le travailleur social du patient et, de surcroit, est imposée au patient qui ne peut, au risque de désenchanter la relation, se prononcer sur ce rapprochement» (2012: 89). Cette observation, qui traduit des rapports de force sur fond d'asymétrie liée à l'exercice des rôles sociaux, touche clairement à la posture professionnelle et à l'éthique.

Les valeurs de préférences, aussi louables soient-elles, ne peuvent à elles seules fonder une posture éthique. Au contraire, une réflexion professionnelle qui se veut soucieuse du bien-être des bénéficiaires, de leur pouvoir d'autodétermination, engage les praticien.ne·s à repérer les dilemmes et valeurs en concurrence dans chaque situation d'intervention ainsi que les prévisibles effets produits par leur positionnement. A mon sens, en cours d'action, l'adoption d'une «attitude esthétique» (Stolnitz, 2004), distincte du regard ordinaire fonctionnel et normatif, devrait venir soutenir un positionnement éthique qui suppose de composer avec l'incertitude (Malherbe, 200I) et de mettre en délibération avec autrui les conflits liés aux valeurs et positionnements.

Oser se confronter à l'ambiguïté et accorder poids à la dimension sensible et non prévisible de l'agir relève d'une conception de la compétence professionnelle malheureusement peu en phase avec l'idéologie positiviste qui sous-tend les approches managériales qui, dans une large mesure, définissent aujourd'hui le travail social (Dujarier, 20I5).

\section{Posture professionnelle et enseignement}

Les précédents développements ont permis de montrer que la posture de recherche qu'implique le recours à la méthode de l'observation participante offre une base de réflexion heuristique pour penser la professionnalité des intervenant·e.s sociaux et, plus précisément, les notions de posture professionnelle et de réflexivité. Aussi, dans le cadre de mon enseignement, tant en formation initiale que continue - formation des superviseur.e.s, notamment - je fais souvent appel à mes observations de chercheur afin de souligner la place centrale qu'occupent le corps et les émotions dans l'agir. Dans un cours qui s'intéresse à penser la question de l'exclusion 
et de l'intégration en rapport au handicap, je n'hésite pas à proposer aux étudiant.e.s de déambuler en fauteuil roulant dans les rues de la cité, exercice suivi d'une seconde expérience qui consiste à se balader à l'aveugle avec un bandeau placé sur les yeux. Sans minimiser les enjeux psychologiques, les impressions vécues en situation sont examinées et analysées en termes somatiques (rupture des perceptions habituelles, rupture d'équilibre, sentiment d'insécurité, etc.) et en termes sociaux (regard public, stigmatisation, assignation de place, etc.). Dans un enseignement qui vise à interroger les représentations sociales du handicap, ce type d'expérience favorise grandement la mise en lumière des mécanismes à l'œuvre entre le groupe majoritaire et les minorités, tout en contribuant à thématiser la posture à tenir pour les intervenant.e.s auprès de ces minorités. Il n'est pas rare que les étudiant·e•s, même celles et ceux qui ont déjà acquis une solide expérience dans l'accompagnement des personnes en situation de handicap, fassent état de leur étonnement. Eprouver dans sa chair le regard des passant.e.s peut amener les participant.e.s à examiner sous un jour nouveau la notion d'empathie, en considérant la complexité de ce mouvement d'ouverture vers l'autre qui peut, paradoxalement, cacher un mécanisme de mise à distance non consciente: l'autre, en tant qu'elle ou il, m'émeut par sa différence, n'est-elle ou il pas, de ce fait même, résolument distinct.e de moi?

Dans le cadre d'un enseignement consacré à la médiation artistique - photographie, théâtre, arts plastiques, etc. - les étudiant.e.s apprivoisent l'«outil» avant de l'expérimenter en termes d'appui à la relation interindividuelle ou groupale. Une pédagogie qui recourt à la création et à l'expérimentation privilégie une perspective esthétique de l'humain, qui englobe les sphères corporelle, émotionnelle et cognitive pour ne pas opposer les activités artistiques et culturelles au travail intellectuel. Pour Martha Nussbaum, les pratiques artistiques sont particulièrement propices au développement d'un soi imaginatif et critique car elles permettent «de cultiver la sympathie conjointement avec le développement des facultés logiques" (20I I : I32). Si le théâtre invite à porter attention au corps et aux émotions, c'est aussi le cas de la pratique du dessin et de la peinture, qui repose sur le lien intime entre le regard et la main (Merleau-Ponty, I964), ou de la photographie, qui rend attentif ou attentive au cadrage du réel, à sa modalisation, c'est-à-dire à la manière de projeter une signification dans les situations d'interaction (Goffman, I99I).

D'une façon ou d'une autre, les pratiques artistiques, entrevues sous l'angle de la médiation de la relation, constituent un terrain favorable pour 
penser le rapport au corps, le rapport aux autres et au monde et, par làmême, la posture professionnelle (Loser, 20I4). Ce dernier point me conduit à considérer les enseignements professionnels situés à l'articulation de la formation théorique et pratique. Dans le suivi des étudiant.e.s en emploi, apprenant.e.s qui exercent une activité dans un lieu reconnu du travail social, la notion de posture professionnelle se présente comme une question incontournable qui demande à être travaillée concrètement à l'aide des analyses de situations professionnelles, des jeux de rôles et de la mise en récit des pratiques. Dans le cadre de cet accompagnement, j'ai pu vérifier de façon renouvelée que le fait d'amener les étudiant.e.s à porter attention à leur positionnement (intention poursuivie, attitude, place occupée, gestes accomplis, paroles prononcées, etc.) en rapport aux effets produits en situation chez eux et chez les bénéficiaires (impact émotionnel, réactions, interactions, etc.) leur permet de mieux saisir ce que recouvre la notion de posture professionnelle. Pour Richard Shusterman (2007), afin de favoriser une prise de distance critique vis-à-vis des émotions, voire autoriser des stratégies à même de les influencer, il s'agit préalablement d'être à même de les repérer et de les thématiser. Pierre Vermersch (I994) préconise de s'intéresser à l'expérience corporelle, qui favorise grandement ce processus de conscientisation des émotions et des ajustements automatiques en situation. Comme mentionné plus haut, une telle démarche est propice à un «devenir conscient» de ses préjugés sociaux via l'examen des situations ambiguës, des situations d'embarras. Pour les praticien·ne·s en travail social, cela revient à s'intéresser à la manière dont les émotions qui surgissent en cours d'interactions peuvent traduire une gêne liée à leurs préférences en termes de valeurs, à leur positionnement social (rapports de force, asymétrie de rôles, etc.) et à leur perception des différences (de genre, de milieu socioculturel, etc.). Cette attention au vécu corporel, qui suit un processus dynamique, relève d'une éthique contextuelle car «le relief émotionnel n'est jamais dicté a priori, mais est toujours le fruit d'une appréciation à chaque fois renouvelée du sujet en jeu » (Depraz, 2006: 93).

\section{Conclusion}

Alors que les praticien.ne.s en travail social sont appelé.e.s dans leur activité quotidienne à se positionner et à faire preuve de réflexivité, il me semble que leur activité pourrait tirer avantage de la méthode ethnographique. 
Au lieu d'insister sur l'impossible neutralité axiologique - la fameuse distance professionnelle -, cette approche postule au contraire que tout être humain est un être incarné, socialisé et «affecté» par les autres et les interactions dans lesquels il est engagé. Ce postulat suppose de ne pas circonscrire les interactions aux seules dimensions affective et interpersonnelle, mais d'appréhender ces dernières aussi comme des situations sociales traversées par des rapports de force et des jeux de pouvoir. Dans cette idée, les praticien.ne.s sont invité.e.s à porter attention aux mouvements corporels et émotionnels, les leurs et ceux repérés chez les bénéficiaires, afin de les consigner par écrit, opération d'objectivation suivie par des analyses qui portent sur les enjeux précités.

A la différence de la recherche, l'intervention sociale n'a pas pour fonction de produire de la connaissance, mais la méthode ethnographique peut assurément aider à décrypter dans leur complexité les situations et les interactions entre protagonistes, et ainsi éclairer les professionnel.le·s quant au positionnement à tenir.

Avec son jeu de dédoublement entre un dedans et un dehors et la pratique du journal de terrain qui rend possible une mise en intelligibilité élargie des interactions entre protagonistes, y compris l'observatrice ou l'observateur, la méthode ethnographique ouvre une piste particulièrement fructueuse pour le travail social car elle permet de penser les notions de réflexivité et de posture professionnelle à la croisée du sensible et du cognitif, du préréflexif et du réflexif.

\section{Références bibliographiques}

Autès, M. (20I3). Le travail social: art de dire, art de faire. Dans Y. Boudjenaï (Dir.), Quel devenir pour le travail social: Nouvelles questions, nouvelles légitimités (pp.43-54). Paris: L'Harmattan.

Avril, C. Cartier, M. \& Serre, D. (2010). Enquêter sur le travail: Concepts, méthodes, récits. Paris: La Découverte.

Boujut, S. (2005). Le travail social comme relation de service ou la gestion des émotions comme compétence professionnelle. Déviance et Société, 29, I4I-I53.

Bourdieu, P. (I980). Le sens pratique. Paris: Minuit.

Capul, M. \& Lemay, M. (2005). De l'éducation spécialisée. L'éducation spécialisée au quotidien. Ramonville Saint-Agne: Erès.

Canter Kohn, R. \& Nègre, P. (2003). Les voies de l'observation. Paris: L'Harmattan.

Caratini, S. (2004). Les non-dits de l'anthropologie. Paris: Presses Universitaires de France.

Certeau, M. de (I990). L'invention du quotidien. I. Arts de faire. Paris: Gallimard. 
I49 | La posture professionnelle et la réflexivité en travail social...

Clifford, J. (2003). De l'autorité en ethnographie. Le récit anthropologique comme texte littéraire. Dans D. Céfaï (Dir.), L'enquête de terrain (pp.263-294). Paris: La Découverte.

Clot, Y. (I998). Le travail sans l'homme? Pour une psychologie des milieux de travail et de vie. Paris: La Découverte \& Syros.

Corcuff, P. (I998). Justification, stratégie et compassion: apport de la sociologie des régimes d'action. Correspondances: Bulletin d'information scientifique de l'Institut de Recherche sur le Maghreb Contemporain à Tunis, 5I, I-9.

Crawford, M. B. (20I0). Eloge du carburateur: essai sur le sens et la valeur du travail. Paris: La Découverte.

Damasio, A. (200I). L'erreur de Descartes. Paris: Odile Jacob.

Dejours, C. (2003). L'évaluation du travail à l'épreuve du réel. Critique des fondements de l'évaluation. Paris: INRA.

Depraz, N. (2006). Comprendre la phénoménologie. Une pratique concrète. Paris: Armand Colin.

Despret, V. (200I). Ces émotions qui nous fabriquent: ethnopsychologie des émotions. Paris: Seuil.

Detrez, C. (2002). La construction sociale du corps. Paris: Seuil.

Dujarier, M.-A. (20I5). Le management désincarné. Paris: La Découverte.

Enthoven, R. (Dir.) (2009). La création. Paris: Perrin.

Favret-Saada, J. (I990). Etre affecté. Gradhiva: revue d'histoire et d'archives de l'anthropologie, 8, 3-9.

Garfinkel, H. (2007). Recherches en ethnométhodologie. Paris: Presses Universitaires de France.

Gaspar, J.-F. (20I2). Tenir! Les raisons d'être des travailleurs sociaux. Paris: La Découverte. Gebauer, G. \& Wulf, C. (2004). Jeux, rituels, gestes. Les fondements de l'action sociale. Paris: Economica.

Goffman, E. (I973). La mise en scène de la vie quotidienne. I. La présentation de soi. Paris: Minuit.

Goffman, E. (I974). Les rites d'interaction. Paris: Minuit.

Goffman, E. (I99I). Les cadres de l'expérience. Paris: Minuit.

Hall, E. T. (I984). La danse de la vie. Paris: Seuil.

Heinich, N. \& Shapiro, R. (Dir.) (20I2). De l'artification: enquêtes sur le passage à l'art. Paris: EHESS.

Henry, M. (2004). Auto-donation. Entretiens et conférences. Paris: Beauchesne.

Henry, M. (2006). Philosophie et phénoménologie du corps. Paris: Presses Universitaires de France.

Joas, H. (200I), La créativité de l'agir. Dans J.-M. Baudoin \& J.Friedrich (Dir.), Théories de l'action et éducation (pp. 27-44). Bruxelles: De Boeck \& Larcier.

Joas, H. (2008). La créativité de l'agir. Paris: Cerf.

Kaufmann, J.-C. (I995). Corps de femmes, regards d'hommes, sociologie des seins nus. Paris: Nathan.

Kaufmann, J.-C. (I997). Le cœur à l'ouurage. Théorie de l'action ménagère. Paris: Nathan. Lahire, B. (200I). L'homme pluriel. Les ressorts de l'action. Paris: Hachette Littérature, Armand Collin/Nathan. 
Le Breton, D. (I990). Anthropologie du corps et modernité. Paris: Presses Universitaires de France.

Le Breton, D. (2007). En souffrance. Adolescence et entrée dans la vie. Paris: Métaillé.

Le Breton, D. (2013). L’adieu au corps. Paris: Métaillé.

Loser, F. (20I0). La médiation artistique en travail social. Enjeux et pratiques en atelier d'expression et de création. Genève: Editions ies.

Loser, F. (20I2). Le journal de terrain selon une perspective esthétique ou quand la pensée se fait sensible. Dans A. Gohard-Radenkovic, S. Pouliot \& P. Stalder, Le journal de bord: un récit en soi ou les traces d'un cheminement (pp.73-IOI). Berne: Peter Lang.

Loser, F. (2013). L’agir des éducateurs sociaux comme expérience esthétique [Thèse de doctorat en Sciences de l'éducation, soutenue le 2I janvier 2013]. Lyon: Université Lumière Lyon II.

Loser, F. (20I4). Esthétique et médiation créative: enjeux et pistes d'action pour interroger les préjugés et soutenir une communication interculturelle.

Dans: P. Stalder \& A. Tonti (Dir.), La Médiation (inter)culturelle: représentations, mises en œuvre et développement des compétences (pp. I29-I5I). Paris: Editions des archives contemporaines.

Loser, F. \& Waldis, B. (2017). Ambiguïté des cadres d'expérience dans les interactions de face-à-face entre personnes en situation de handicap et personnes «valides»: quelles implications pour les chercheurs? Dans: S. Garneau \& D. Namian, Erwing Goffman et le travail social (pp. I97-2 I2). Ottawa: Presses de l'Université d'Ottawa. Malherbe, J.-F. (200I). L'incertitude en éthique: perspectives cliniques. Montréal: Fides. Mauss, M. (200I). Sociologie et anthropologie. Paris: Presses Universitaires de France. Mead, G. H. (2006). L'esprit, le soi et la société. Paris: Presses Universitaires de France. Mercadier, C. (2006). Le travail émotionnel des soignants à l'hôpital. Paris: Seli Arslan. Merleau-Ponty, M. (I964). L'œil et l'esprit. Paris: Gallimard.

Molinier, P. (2013). Le travail du care. Paris: La Dispute.

Nussbaum, M. (20I I), Les émotions démocratiques: Comment former le citoyen du $2 \mathrm{I}^{e}$ siècle? Paris: Flammarion.

Ravon, B. \& Ion, J. (20I 2). Les travailleurs sociaux. Paris: La Découverte.

Schön, D. A. (1994). Le praticien réflexif: à la recherche du savoir caché dans l'agir professionnel. Montréal: Logiques.

Sennett, R. (2010). Ce que sait la main: La culture de l'artisanat. Paris: Albin Michel.

Shusterman, R. (2007). Conscience du corps. Pour une soma-esthétique. Paris/Tel Aviv: Editions de l'éclat.

Stolnitz, J. (2004). L'attitude esthétique. Dans D. Lories (Dir.), Philosophie analytique et esthétique (pp. I03-I I4). Paris: Klincksieck.

Vermersch, P. (1994). L'entretien d'explicitation. Paris: ESF.

Winkin, Y. (200I). Anthropologie de la communication. De la théorie au terrain. Paris: Seuil. Winnicott, D. W. (1975). Jeu et réalité: l'espace potentiel. Paris: Gallimard. 


\section{Faire se rencontrer recherche, intervention et formation}

Effets en cascade d'un projet de recherche

Sylvie Mezzena et Kim Stroumza

\section{Introduction}

Nous souhaitons discuter la portée de nos recherches en analyse de l'activité (Barbier \& Durand, 20I7) à l'intérieur de nos cours et "dans la cité», par-delà les murs de notre école, en présentant comment nos projets de recherche génèrent différents effets scientifiques, pratiques et pédagogiques, avec des conséquences qui ne se limitent pas aux enseignements dans la formation professionnelle initiale. Ces effets permettent de relier la recherche à d'autres champs d'activités comme la formation postgrade interne ou transverse à un champ de pratiques, l'accompagnement d'équipe ou de direction, ou encore la conception d'outils de formation à visée pédagogique ou de développement professionnel.

Si de tels usages dérivés de la recherche et leurs répercussions sont possibles, c'est parce que nous ne nous limitons pas à une vision et à une définition de la recherche qui la cantonneraient à des cloisonnements distinguant d'une part recherche fondamentale et d'autre part recherche appliquée, ou encore recherche d'un côté et intervention sur les terrains de l'autre. Il faut d'emblée préciser que notre manière de considérer la recherche est issue de sa pratique même, qui, au fil du temps, a eu pour conséquences de nous ouvrir à des opportunités et des possibles qui nous ont amenées à dépasser ces oppositions et à remettre en question une certaine vision de la recherche appliquée, et peut-être plus largement de la recherche.

Pour ne pas restreindre notre propos à une discussion purement théorique ou épistémologique, voire idéologique, et bien souligner que notre position scientifique a à voir avec la pratique même de la recherche, nous allons illustrer comment un projet de recherche peut rendre possibles différents déploiements en considérant un exemple concret. L'intérêt de 
notre exemple est que cette recherche est elle-même issue d'une première intervention à la demande du terrain, ce qui nous semble bien caractériser une façon possible de faire de la recherche dans les hautes écoles. ${ }^{1}$ Il s'agit d'une recherche menée en partenariat avec le secteur de la petite enfance de Vernier, ${ }^{2}$ visant la visibilisation du savoir-faire dans les pratiques éducatives quotidiennes favorisant l'intégration. Ce volet des activités professorales dans les hautes écoles pose la question du lien plus ou moins serré aux terrains, qui habite en arrière-fond les débats sur la recherche et son orientation ou devenir dans les écoles professionnalisantes de niveau tertiaire.

Après avoir rapidement présenté le paysage dans lequel nous situons notre démarche (aussi bien dans le champ des recherches partenariales que dans celui de l'analyse du travail), nous allons présenter le contexte d'émergence de ce projet ainsi que ses particularités, notamment scientifiques, avec notre manière de faire de l'analyse de l'activité, mais sans trop nous attarder sur les contenus des résultats obtenus à cette occasion (Mezzena, Stroumza \& Borel, 2014a; Mezzena, Stroumza \& Borel, 20I4b). Nous allons nous concentrer davantage sur la portée de cette démarche aux différents niveaux précités, en montrant comment cette rencontre entre recherche, intervention sur des terrains, formation, valorisation scientifique et conception d'outils de formation génère des effets qui transforment mutuellement ces champs d'activité, en permettant notamment de considérer autrement la recherche et ses possibles, et plus largement d'ouvrir de nouvelles perspectives pour les actrices et acteurs engagé.e.s dans ce partenariat.

\section{Un positionnement en analyse du travail, avec une orientation pragmatiste}

\section{Construire des alternatives au modèle}

de la diffusion/application de connaissances

De nombreux travaux scientifiques développent des modèles alternatifs au modèle applicationniste, dit encore modèle de la diffusion ou du transfert de connaissances (voir Stroumza \& Mezzena, 20I6; Mezzena et al., 20I4; Lyet \& Paturel, 20I 2; le dossier de la revue SociologieS, 20I I; Gillet, 20I7). Ce modèle classique et linéaire conçoit la recherche appliquée 
comme l'opérationnalisation, dans des environnements définis, de connaissances produites en recherche fondamentale. Les résultats produits par les chercheur.e.s sont transférés, diffusés, en vue d'être appliqués par leur public-cible. Le processus de fabrication des savoirs dans l'activité scientifique est considéré comme préalable et indépendant du processus de diffusion et de transformation visé. Dans cette optique, si le publiccible n'est pas preneur des résultats, c'est soit que la science a mal communiqué, soit que le public-cible résiste, prisonnier de ses préjugés, désirs, illusions, voire valeurs; public-cible qu'il s'agit alors de ramener à la raison en tentant de lever cette résistance au changement (Stengers, I997; Jonckheere, 20I0).

Dans un premier projet consacré aux processus mis en œuvre dans l'activité partenariale entre chercheur·e.s et leurs partenaires (Seferdjeli \& Stroumza, 20I I), nous avions pris pour objet des recherches réalisées dans notre contexte national et dans les hautes écoles spécialisées suisses (HES). Nous avons ainsi pu mettre en évidence, pour l'essentiel, des éléments constitutifs de ce processus et caractéristiques des propriétés de toute activité: l'incertitude, la confiance et l'engagement. L'engagement dans le processus de partenariat est décrit comme un saut dans l'incertain, les protagonistes doivent faire face à un développement imprévisible des circonstances et s'exposer à des conséquences qu'elles et ils ne peuvent spécifier. Le contexte est mouvant, il oblige chacun.e à s'ajuster constamment pour pallier les différents incidents. Les partenaires «font confiance au processus de travail qu'ils établissent en commun" (Béguin \& Cerf, 2009), au sens où ils ont «confiance dans le devenir scientifique d'une telle relation" (Thiery \& Cerf, 2009). Contrairement à ce que dessine le modèle de la diffusion, le processus n'est pas linéaire et les protagonistes ne sont pas passifs, car l'action n'est pas la simple exécution d'un plan (Suchman, 1987). Pour faire face au développement imprévisible du processus, des ressources diverses et hétérogènes sont puisées tant dans l'expérience ordinaire que dans la boîte à outils de la démarche scientifique.

1 Celle-ci a en effet pour caractéristique d'enjoindre les professeur.e-s à mener en parallèle des prestations sur les terrains et des projets de recherche.

2 Recherche intitulée "Travail d'intégration à l'œuvre dans l'activité des éducatrices et éducateurs de l'enfance de la Commune de Vernier ", menée en 2013 à la suite d'une première prestation qui s'est déroulée en 2012. 
Nous allons voir concrètement que notre manière de faire de la recherche est le fruit d'un agencement entre activité scientifique et participation active des partenaires, et ce dans un contexte sociopolitique propre à l'institution et son champ de pratiques, en ne dissociant pas ces dimensions et en les inscrivant comme exigences scientifiques (Seferdjeli \& Stroumza, 20I7). Nous tentons ainsi de sortir d'une conception des résultats scientifiques qui auraient le statut de prescription/recommandation à l'égard des pratiques, ou encore de fondements épistémiques, censés refléter une vérité qui devrait guider les pratiques.

Pour ce faire, nous adoptons un positionnement au sein de l'analyse du travail, avec une orientation pragmatiste qui requiert: une participation des partenaires exigée par les spécificités de l'objet de la recherche, ainsi que le développement de dispositifs de recherche qui fabriquent/suscitent de l'intérêt; la reconnaissance d'une connaissance pratique non assujettie à une forme objectiviste de connaissance qui lui serait supérieure, en considérant l'existence de connaissances dans les pratiques cibles. Celles-ci ne sont alors pas de simples réceptacles passifs des résultats scientifiques.

\section{Un positionnement en analyse du travail}

Dans le large champ de l'analyse du travail, les travaux se sont développés à partir de la distinction fondatrice entre travail prescrit et travail réel de l'ergonomie traditionnelle de langue française et depuis une critique d'une conception rationnelle, planificatrice et applicationniste de l'action. Dans le travail social également, de nombreuses recherches s'inscrivent à leur suite (par exemple, Jonckheere, 2010; Libois, 2013; Kostulski, Clot, Litim \& Plateau, 20I I). Ces travaux montrent que lorsque la ou le professionnel.le agit, elle ou il ne peut se contenter d'appliquer des prescriptions, des théories ou de mettre en œuvre des politiques, elle ou il s'achoppe au réel. Ce travail dit réel comporte un travail interne d'organisation, qui se déroule dans le temps, et s'organise «dans et par le moyen" de l'environnement (Quéré, 2006). L'activité suit ainsi une logique propre, que l'ethnométhodologie conçoit comme un raisonnement pratique (Fornel, Ogien \& Quéré, 200I), le courant de l'énaction comme un couplage organisme/environnement avec une auto-organisation endogène (Durand, 2008), le pragmatisme comme une transaction organisme-environnement qui vise un équilibre (Dewey, I993 [1938]). Pour analyser une activité et reconstruire sa logique propre, il faut dès lors «examiner la manière dont elle se réalise 
[dans une situation], sans faire de cette réalisation le produit nécessaire d'un déterminisme ou d'une rationalité „33 (Ogien \& Quéré, 2005: 3). Dans ces travaux, le lien entre action et situation n'est pas contingent, mais constitutif de l'agir (Quéré, 2006).

Ces travaux identifiés comme relevant d'une approche située ou contextualisée montrent que si les prescriptions, les discours politiques, les idées, les facteurs sociaux, les normes, les usagères et usagers, l'espace, le temps, ou encore la culture, le psychisme et la personnalité des protagonistes sont bien des éléments qui influencent l'activité, ils ne l'influencent cependant pas de manière déterministe ou causaliste. Ces éléments agissent solidairement les uns sur les autres pour constituer l'activité (celle-ci n'est pas issue de l'un ou de l'autre seulement) et sont ainsi mutuellement transformés par cet agencement. Et les professionnel-le·s sont pris dans ce partenariat avec l'environnement, elles et ils sont actifs aux côtés de ces autres "facteurs", elles et ils ne "font pas à cause des déterminismes, elles et ils font avec» (Despret \& Porcher, 2007). Les professionnel.le·s ne sont donc pas tout-puissants pour dicter le déroulement de l'activité. Ce n'est ni leur bonne volonté ni leur réflexion qui sont les seules causes d'influence de l'activité et les garants d'une activité de qualité. Interviennent également leur perception, leur attention, leurs émotions ainsi que tous les autres éléments de l'environnement.

Les professionnel-le.s doivent faire avec ces autres éléments qui affectent le déroulement de l'activité, et ce «faire-avec» affecte en retour ces éléments, les réoriente afin que les professionnel.le·s puissent tenir le cap de leur mission et obtenir dans la durée un équilibre dans ce partenariat avec l'environnement. C'est dans ce «faire-avec» les autres éléments que se loge le savoir-faire professionnel (Mezzena, 20I4).

Si les professionnel.le.s savent faire, ils ne savent pas forcément dire ce savoir-faire, parce que celui-ci engage le corps, les émotions, la perception, l'attention. Il y a là un savoir-faire phénoménal, expérientiel, à saisir dans le fonctionnement même de l'activité et qui ne dépend ni d'une activité

Non pas que l'action serait irrationnelle: «rationaliste», ici, se comprend au sens intellectualiste de ce qui découle d'un raisonnement trouvant son lieu de procès dans le mental, qui se distingue de ce qui relève d'une logique rationnelle trouvant son fondement dans le déroulement interne de l'action, depuis les conséquences générées au fil de son procès. 
intellectuelle (par exemple, définie comme travail de réflexion ou de réflexivité) qui serait additionnelle à la pratique selon la légende intellectualiste (Ryle, 2005) ni comme une activité psychique interne (cf. les travaux de cognition distribuée). Si les travaux d'analyse du travail insistent sur le fait que le point de vue des professionnel.le.s est nécessaire à la description de leur savoir-faire (c'est ainsi une exigence de leur objet d'étude, Mezzena, Seferdjeli \& Stroumza, 20I4), ils affirment cependant également qu'il ne suffit pas d'interroger les professionnel-le.s pour y avoir accès. Pour accéder à la logique propre de l'activité et à la manière dont les professionnel-le·s l'investissent, ces travaux ont développé tout un ensemble de méthodes (entretiens d'instruction au sosie, d'explicitation, d'autoconfrontation). Il s'agit d'éviter les discours d'intention, les généralisations ou les justifications en rapprochant, dans le temps même de l'entretien, le ou la professionnel.le de son activité réelle à l'aide de traces de l'activité réelle et/ou d'un questionnement particulier.

Tout en s'inscrivant à la suite de cet ensemble de travaux, nous avons été amenées ${ }^{4}$ à développer plus spécifiquement une orientation pragmatiste, dans la suite des travaux de Jonckheere (2010), Ogien \& Quéré (2005), Despret (2009) et Stengers (I997), eux-mêmes dans le sillage des philosophes pragmatistes américains James (2007) et Dewey (I993 [I938]; 20I I).

\section{Une orientation pragmatiste}

Pour décrire plus précisément ce savoir-faire phénoménal, expérientiel, nous avons repris à Dewey la notion d'enquête, en l'opérationnalisant depuis les travaux de la sociologie de l'action et en l'enrichissant d'autres apports du pragmatisme. Ce savoir-faire avec les autres éléments de l'environnement est alors saisi comme un processus de partenariat avec l'environnement, qui s'appuie sur de l'anticipation, de l'appréciation et de l'expérimentation, en développant une perspective pratique inscrite dans la continuité de l'expérience et inscrivant le guidage de l'activité dans le cumul des effets produits par la poursuite de la perspective (Mezzena, à paraître).

Comme le soulignent Lyet et Paturel (2012), c'est la question épistémologique qui valide et rend cohérente l'intégration de l'activité des professionnel.le.s dans le processus de recherche. Dans nos recherches, nous n'adoptons pas une conception transcendantale ou intellectualiste de la connaissance, présupposant que les connaissances préexistent à notre 
expérience et la prédéterminent totalement, et impliquant de retrouver cette connaissance qui serait appliquée par les professionnel.le.s. Plus précisément, nous n'adoptons pas une définition de la connaissance qui loge celle-ci dans la ou le professionnel.le (qu'il s'agisse de sa subjectivité, de sa rationalité ou réflexivité, de ses dispositions personnelles comme la personnalité ou les valeurs) ou dans des entités qui surplomberaient l'expérience en la prédéfinissant (la Raison, les théories, la culture, ou encore la connaissance scientifique au sens classique). Nous n'optons pas pour une conception de la connaissance comme reflet d'un monde déjà donné, réalité déjà toute construite et en attente d'être observée, analysée et révélée depuis une position d'extériorité et de surplomb (d'objectivité). Les professionnel.le.s ne travaillent pas avec des situations déjà données, toutes constituées, mais font avec des forces dans des situations qui se construisent en cours d'activité. Même si leurs constructions comportent des régularités, les professionnel.le.s n'échappent pas à l'obligation de créer un savoir-faire toujours susceptible d'évoluer au fil de la mutation des problèmes. Depuis notre épistémologie pragmatiste, nos recherches portent sur des réalités (du monde) qui se construisent incessamment, depuis nos transactions avec le monde et ses objets. La connaissance participe de cette construction du monde, tout en étant construite par elle. Ainsi, la connaissance accompagne plutôt qu'elle ne fonde la construction du monde (Jonckheere, 2010; Mezzena, 20I4). ${ }^{5}$ Et quand cette connaissance porte sur les pratiques professionnelles, elle est construite avec les professionnel-le.s.

Méthodologiquement, nous utilisons notre dispositif de recherche pour fabriquer, susciter de l'intérêt (Despret, 2009). Il ne s'agit ainsi pas, dans un souci épistémologique de rupture avec le sens commun et de purification scientifique, de tenter de réduire ou gommer une implication de la part des professionnel.le.s ou des chercheur.e.s. Mais, à l'opposé, il ne s'agit pas non plus, depuis une position uniquement politique ou idéologique, de rendre le dispositif le moins asymétrique possible en vue de soutenir une participation démocratique des professionnel.le·s à la démarche

4 Pour une présentation de notre évolution théorique au fil de nos dix années de recherches communes, cf. Mezzena \& Stroumza, à paraître.

5 Ce rapport à la connaissance se retrouve également dans les travaux de chercheur.e.s de diverses disciplines, par exemple, Hennion en sociologie, Debaise et Soldhju en philosophie. 
scientifique. S'il importe d'inviter les professionnel.le·s à décrire leur savoirfaire depuis ce qui les intéresse, c'est parce que c'est la condition pour qu'elles et ils puissent mettre en rapport leurs idées avec nos questions et nos observations, que celles-ci leur permettent d'emprunter les chemins d'idées qui rendent compte au mieux des réalités de leur pratique. Selon quelles suites d'idées cheminent-elles et ils pour décrire leur travail d'enquête comme investigation, recherchant certains effets et anticipant certains risques sur le territoire pratique qui est le leur? Depuis quelle recherche d'effets construisent-elles et ils leurs problèmes et guident-ils leurs activités? Quelle logique pratique d'ensemble, quel modèle d'intervention guide leur savoir-faire? L'enjeu est que nous, chercheur.e.s, puissions les suivre en pensant avec elles et eux au milieu de leurs pratiques, depuis l'environnement dans et par lequel ils construisent leurs activités, en tentant de suivre (le point de vue de) leur perspective. Le dispositif doit pouvoir favoriser ces mises en rapport d'idées avec des faits, des évènements, pour leur permettre de décrire finement les variations subtiles des situations et les agencements différents qu'exige l'activité.

D'un point de vue scientifique, il s'agit donc de prendre activement en considération la manière dont les professionnel-le.s prennent activement en compte nos questions ainsi que le dispositif: non pas comment elles et ils s'y soumettent en préjugeant que nous savons à quelle interprétation de la question elles et ils répondent quand elles et ils nous répondent, mais en essayant de saisir ce qui importe pour elles et eux (et à quoi elles et ils répondent quand elles et ils nous répondent). Les problèmes construits par les chercheur.e.s, ainsi que les solutions qu'elles et ils fabriquent, doivent trouver l'intérêt des professionnel.le.s (Despret \& Porcher, 2007), au sens où elles et ils doivent rendre compte des difficultés et du plaisir du travail d'enquête (la dimension phénoménale, expérientielle des activités), avec le pari que c'est là où les professionnel.le.s sont intéressé.e.s que les problèmes sont les plus intéressants. Qu'elles et ils choisissent telle séquence pour une autoconfrontation, qu'elles et ils délaissent certaines de nos questions, qu'elles et ils se passionnent pour d'autres, nous renseigne sur notre objet, sur ce qui leur importe. C'est donc à nouveau notre objet qui nous impose d'être attentives aux effets de nos démarches: comment nos questions affectentelles les professionnel.le.s, et aussi, comment sont-elles affectées par elles et eux, et à quelles nouvelles pistes de compréhension amènent-elles?

Susciter, favoriser un intérêt chez les professionnel.le·s - orienté.e.s vers une connaissance de l'activité et son développement - est ainsi une 
condition pour adopter, saisir leur perspective et ainsi modéliser leur savoir-faire; mais c'est aussi une condition pour produire une connaissance avec les professionnel.le.s, pour faire connaissance avec elles et eux (Stroumza \& Mezzena, 20I6), au sens d'expérimenter à l'occasion du dispositif de recherche des mises en rapport d'idées nouvelles permettant d'expérimenter autrement les problèmes et leur construction, en ouvrant à de nouvelles conséquences elles-mêmes sources de nouveaux possibles. Bref, comment le dispositif de recherche fait-il exister la possibilité d'ouvrir à de nouvelles expériences?

De plus, si nos recherches produisent de la connaissance et des transformations (Durand, 2008), nous ne visons pas à produire tel ou tel effet chez les professionnel.le.s (par exemple, en termes de prise de conscience ou d'amélioration des pratiques). Le dispositif de recherche est activement orienté vers la fabrication d'intérêt pour les professionnel-le·s (et les chercheur·e·s), mais sa nature et sa forme, de même que son occurrence ou non, sont laissées totalement libres par les chercheur·e.s; nous ne cherchons pas à avoir prise là-dessus. Contrairement à d'autres démarches d'analyse du travail, nous n'intervenons pas spécifiquement dans des situations de travail jugées dégradées, ou pour régénérer un métier en panne de controverses. Nos interventions visent à construire une connaissance des activités, et c'est aux professionnel-le.s de se saisir (ou non) du dispositif de recherche pour faire (ou non) évoluer leurs pratiques. En revanche, notre démarche de recherche est non seulement suffisamment malléable, mais également à l'affût de ce qui peut intéresser les professionnel-le·s, ce qui permet au dispositif même de se transformer, de s'ajuster à et de se nourrir de ces différents intérêts (tout en ne lâchant pas notre intérêt de chercheur.e.s).

Nous allons maintenant voir concrètement, à partir du récit d'une démarche avec le service de la petite enfance de la Ville de Vernier, comment intérêts de chercheur.e.s, intérêts de formatrices et formateurs, intérêts de professionnel.le·s du champ de la petite enfance se sont mutuellement fécondés (et continuent à le faire) depuis maintenant déjà cinq années. ${ }^{6}$

6 Notre quotidien de formateur ou formatrice-chercheure à la HETS est ainsi traversé de plusieurs aventures qui se tissent au cours du temps et sont à la fois autonomes les unes des autres (il n'y a d'ailleurs pas toujours les mêmes personnes engagées dans le processus de recherche), et inscrites dans une visée programmatique (cf. infra). 


\section{Récit d'un partenariat avec le service de la petite enfance de la ville de Vernier}

D’une prestation en analyse de l'activité à un projet de recherche

La demande de prestation qui nous a été adressée au départ était de visibiliser le savoir-faire à l'œuvre dans les pratiques éducatives du point de vue du travail d'intégration, depuis l'approche de l'analyse de l'activité. Cette prestation a été financée par le Bureau de l'intégration des étrangers (BIE) du Canton de Genève. Ce financement a été obtenu par un groupe de travail interne au Service de la petite enfance (SPE) de la Ville de Vernier, dévolu à des projets en faveur de l'intégration et composé de la direction générale, de plusieurs directions de structures et d'une éducatrice de la petite enfance (qui se trouve être également enseignante vacataire en analyse de l'activité à la HETS dans des modules de formation sous notre responsabilité). C'est cette petite équipe qui nous a mandaté.e.s ${ }^{7}$ : si une conviction habitait ce groupe de travail sur le fait qu'une prestation de qualité était effectuée en termes d'intégration, la question se posait de savoir de quoi était fait le savoir-faire des éducatrices et éducateurs, celui-ci n'étant pas facile à saisir, à décrire, à argumenter.

La démarche a consisté à décrire finement le travail d'intégration depuis une analyse de l'activité dans trois structures. ${ }^{8}$ Pour les professionnel.le.s de ce terrain, le travail d'intégration peut minimalement être défini ainsi: savoir tenir compte de ce qui importe pour les personnes (professionnel.le.s, parents, enfants). Il s'est avéré non seulement être d'une certaine envergure tout en étant diffus; il a également été souligné que ce travail d'intégration, logé dans les prises en charge quotidiennes, s'adressait à tous les enfants et à tous les parents, sans discrimination (voir Mezzena, Stroumza \& Borel, 20I4a et b).

Les résultats de cette première étape du mandat de prestation ont été présentés aux collaboratrices et collaborateurs administratifs et pédagogiques du SPE de la Ville de Vernier, à l'occasion de conférences qui ont été réitérées plusieurs fois afin de permettre une présentation de la démarche et de ses résultats à l'ensemble du personnel. ${ }^{9}$ Les trois professionnel.le.s qui ont participé à la démarche sont intervenu.e.s à nos côtés pour présenter leur motivation à participer ainsi que ce qu'elles et ils en ont retiré professionnellement. Les conférences ont été suivies de discussions avec 
l'ensemble des professionnel.le.s convié.e.s et des représentant·e.s politiques de la Commune de Vernier, également présent.e.s.

Durant cette première étape d'intervention a été mesurée l'importance du travail d'intégration effectué dans le partenariat avec les familles. Les discussions autour de ce résultat dans le groupe de travail ont été marquées par la reconnaissance des directions, très émues et fières du travail accompli par les professionnel-le·s. Le constat d'un matériel très riche à analyser a également été fait, ce qui a finalement encouragé le groupe à poursuivre notre collaboration, mais cette fois avec un projet de recherche, de nouveau financé par le BIE. L’analyse s'est alors focalisée plus spécifiquement sur le travail d'intégration effectué avec les familles, afin d'apprécier ses rapports avec l'intégration des enfants et d'examiner ses effets pour l'intégration au sens large.

A ce moment-là, nous terminions une recherche consacrée à l'analyse de la réflexivité dans l'activité des éducatrices et éducateurs (Mezzena et al., 20I3) et étions en passe de déposer un nouveau projet au Fonds national suisse (FNS), consacré à la formation des valeurs dans l'activité (Mezzena et al., 20I6a et b). Ce projet sur le travail d'intégration dans les activités des éducatrices et éducateurs de la petite enfance de Vernier a ainsi dialogué avec d'autres travaux en cours et nous a permis tant de consolider notre outillage théorique que d'affiner nos analyses en les confrontant à d'autres données empiriques. Nous avons mesuré combien cette articulation entre différents types de projets, qu'ils soient mandatés et financés par des terrains ou subventionnés par des fonds de recherche, est féconde pour porter

7 L'équipe était composée de Sylvie Mezzena et Kim Stroumza, de Cécile Borel, éducatrice de l'enfance à l'EVE des Libellules et déléguée à la recherche pour le SPE de la Ville de Vernier, et de Pascal Baumgartner (http://www.ikon-productions.ch).

8 Cette recherche a été rendue possible grâce à la participation des trois professionnel.le·s qui ont accepté d'être filmé.e.s: Camilla Gibbons à Eve Lignon, Martine Sabot Landwehr à l'accueil parents-enfants Bourquin et Yoram Prigent au jardin d'enfants Bourquin, que nous remercions pour leur implication. Nous en profitons pour également remercier les familles qui ont accepté que les films réalisés soient diffusés et contribuent à la valorisation du métier et à la formation professionnelle.

9 Jorge Dias Ferreira, psychologue scolaire et adjoint scientifique à la direction générale du DIP, a également apporté sa contribution lors de ces rencontres en amenant des apports sur la question de l'intégration. 
une dynamique cohérente en termes de programme de recherche, tout en diversifiant et en enrichissant nos activités.

La recherche intéresse ces terrains, qui y voient une opportunité de visibiliser leur savoir-faire, malgré un investissement accru en termes d'heures et de budget. Cela implique en effet de mettre au budget des heures supplémentaires pour un travail de valorisation et de publication qui n'est pas présent dans les prestations. De notre côté, une telle occasion a également rencontré notre envie de publier sur des éléments de connaissance nouveaux, tout en répondant à notre obligation professionnelle, en tant que professeures, de valoriser nos travaux. De plus, de tels projets de rechercheintervention nous permettent de recueillir de nouvelles données empiriques, voire de bénéficier de nouvelles ressources pédagogiques selon les conventions négociées avec les terrains. En contrepartie, une part de nos heures sont prises sur un forfait d'heures qui nous sont allouées pour les recherches dans notre feuille de charges.

\section{Du deuxième volet de recherche à la création d'un DVD interactif}

Cette seconde étape a permis la création d'un matériel pédagogique sous forme de DVD, à destination de la formation des collaboratrices et collaborateurs de la petite enfance. Ce double DVD $^{10}$ est interactif, au sens où son usage peut être décidé, orienté par l'utilisateur ou l'utilisatrice de manière différente selon ses besoins, qu'il s'agisse d'un·e formatrice ou formateur ou de participant.e.s à la formation.

L'idée de fabriquer un outil de formation multimédia à destination des professionnel.le.s a émergé en cours de route, depuis le constat d'un matériel très riche valant la peine d'être davantage exploité. ${ }^{11}$ En outre, l'accueil positif de nos présentations dans les conférences a montré que c'est parce que les montages de films présentés étaient accompagnés de nos commentaires d'analyses sur les films qu'ils permettaient aux professionnel.le.s de mettre en lumière et en mots des aspects invisibles et indicibles du métier qu'elles et ils n'arrivaient pas à décrire par eux-mêmes. C'est ainsi qu'est née l'idée de produire un matériel pouvant être utilisé à visée de formation, qu'il s'agisse de perfectionnement pour de nouveaux collaborateurs et collaboratrices dont l'emploi antérieur ne les confrontait pas à la question de l'intégration, ou pour des novices nouvellement arrivé.e.s dans le métier. Si, au départ, ce matériel était uniquement destiné à un usage à l'interne pour les collaboratrices et collaborateurs du SPE de la Ville de 
Vernier, par la suite, au vu de l'intérêt plus général que comportait cet outil pour le champ professionnel de la petite enfance, il a été décidé de le rendre accessible en le diffusant par vente.

Le coffret comprend deux DVD: un premier disque qui se consacre aux activités à l'œuvre dans le travail d'intégration au quotidien et présente des notions de base pour l'analyse de l'activité, notions qui ont évidemment donné une certaine orientation à ce qui a été observé et à la manière dont nous le mettons en évidence; un deuxième disque qui est, lui, consacré à la question de l'intégration dans le secteur de la petite enfance de la Ville de Vernier, avec une volonté, d'un côté de mettre en évidence la spécificité des trois lieux avec lesquels nous avons travaillé, tout en pointant, de l'autre côté, la visée commune à ces terrains qui, en l'occurrence, concerne l'ampleur et la couleur particulière du travail d'intégration effectué en partenariat avec les parents.

La construction de ce double DVD était pour nous une première en termes techniques et nous avons dû apprendre à présenter nos analyses dans ce nouveau format. Il a fallu ainsi tester l'effet de certains films, affiner les montages pour les rendre pertinents, trouver une manière de présenter les principales notions théoriques qui ne soit pas trop rébarbative, imaginer comment inclure dans le DVD des interventions des directions présentant l'historique des institutions et le cadre politique. Tout cela s'est construit très progressivement, par tâtonnements, ajustements, mais porté par un enthousiasme communicatif, un intérêt commun pour la subtilité des pratiques et leur portée politique.

Dans cette période, en parallèle, nous avons publié un premier article, à destination du champ de la petite enfance, dans une revue professionnelle (Mezzena et al., 20I4a) et reçu deux sollicitations pour intervenir dans des colloques scientifiques, ce qui a représenté l'occasion pour nous de tester notre manière de modéliser le savoir-faire en présentant nos travaux dans un autre champ professionnel et de faire paraître une seconde publication (Mezzena et al., 20I4b). La rédaction de ces deux articles a été petite enfance de Vernier: analyse de l'activité. Il est en vente au Service de la petite enfance de Vernier.

11 Le double DVD ne compte en effet pas moins de sept heures de films d'activités et d'autoconfrontations. 
dès le départ pensée comme complémentaire aux DVD, en intégrant ceuxci comme ressources.

\section{Différentes utilisations du double DVD en contexte de formation}

Depuis, cette recherche a connu une portée spécifique dans nos activités d'enseignement, avec un usage des résultats de la recherche et du double DVD dans différents cours de niveaux Bachelor, Master, ou dans des interventions de formation postgrade, par exemple le CAS des praticien.ne.s formatrices et formateurs. Nous l'utilisons avec les étudiant.e.s pour illustrer des notions théoriques, montrer des exemples de pratiques, ou les faire travailler à partir d'une séquence de film accompagnée de consignes pour son analyse, encore reprise avec le visionnement soit des propos de professionnel.le·s, soit des commentaires d'analyse. Nous l'utilisons également pour travailler avec nos collègues formatrices et formateurs qui interviennent dans les enseignements en analyse de l'activité.

Même si les articles sont déjà écrits et qu'il est trop tard pour les enrichir des réactions des étudiant.e.s et de nos collègues, nous nous appuyons néanmoins constamment sur leurs réactions et commentaires pour affiner la façon dont nous travaillons avec nos notions théoriques, et parfois aussi pour développer certaines analyses dans d'autres directions, ce qui permet d'approfondir encore, après coup, notre compréhension de nos données.

Ainsi, nous exploitons ce matériel aujourd'hui encore et c'est une manière pour nous aussi de compenser les nombreuses heures consacrées à cette recherche, au-delà des heures subventionnées. A ce sujet, d'ailleurs, il faut souligner qu'en contrepartie du temps que nous prenons sur nos heures payées par l'école, nous négocions avec les terrains dès le départ cette possibilité pour nous de pouvoir utiliser le matériel produit en formation; ce qui est, nous essayons de le montrer, une condition pour que nos activités puissent se féconder mutuellement.

Le coffret ne propose aucun scénario pédagogique prédéfini, mais se présente sous forme d'une structure arborescente très détaillée comportant différentes entrées avec de nombreuses ressources empiriques et théoriques. Les formatrices et formateurs peuvent ainsi le parcourir au gré de leurs intérêts pédagogiques et construire son usage depuis leurs besoins. Son usage permet ainsi également une diffusion de notre approche en analyse de l'activité auprès de formatrices et formateurs, d'étudiant·e.s et des terrains. 
L’absence de scénario pédagogique inscrit dans les DVD rend, par contre, leur utilisation plus difficile par des formatrices et formateurs qui ne sont pas habitués à travailler à partir de traces d'activités, et également par les directions, lesquelles ne savent pas forcément comment l'exploiter concrètement au sein de leurs réunions d'équipe. Cette difficulté a amené l'ensemble des directions qui ont participé au projet à nous inviter à penser à une suite pour faciliter et accompagner cet outil (voir infra, "Ouverture pour d'autres aventures de construction d'un matériel pédagogique»).

Des interventions sur l'extérieur: Genève, Renens, Paris

Le service d'intégration de la Ville de Renens nous a sollicitées pour offrir une journée de formation à des professionnel-le.s de Renens, qui toutes et tous ont pour mission de mettre en œuvre un programme cantonal d'intégration: ludothécaires, éducatrices et éducateurs dans des foyers, éducatrices et éducateurs du jeune enfant, accueillant.e.s de jour. Travailler ensemble sur des séquences du double DVD ainsi que sur des récits de leur pratique a permis de commencer à construire une certaine interconnaissance entre des institutions fréquentées souvent par un même public, mais dont les professionnel.le.s se côtoient très peu. Cette expérience nous a également amenées à élaborer de nouveaux scénarios pédagogiques, notamment en exploitant la deuxième partie du DVD, qui à la fois souligne les différentes histoires des institutions et, en même temps, les inscrit dans un même territoire et une même politique. A la suite de cette journée, organisée en 2016, il a été décidé de reconduire cette formule pour 2017, mais en augmentant le temps de formation à un jour et demi. De plus, la demande nous a été faite par le service d'intégration de la Ville de Renens de mettre en place une formation spécifique pour les accueillant.e.s de jour.

Nous avons répondu positivement à une invitation de participation à une table ronde organisée par l'équipe de recherche de Laurent Filliettaz, de l'Université de Genève, rassemblant différent.e.s intervenant.e.s du champ de la petite enfance à Genève ainsi que plusieurs chercheur.e.s intéressé·e.s à modéliser leur pratique. En nous appuyant sur l'intervention à Renens, nous avons ainsi pu souligner l'intérêt à faire se rencontrer différents corps professionnels travaillant auprès ou avec des enfants, alimentant ainsi une intervention de Sandra Capeder, cheffe du Service de la petite enfance de la Ville de Genève, appelant le développement d'une 
politique de l'enfance rassemblant plusieurs champs professionnels: éducation à la petite enfance, enseignement, animation parascolaire, ludothèque, éducation en foyer.

Nous avons également présenté ce coffret à l'école Horizon du jeune enfant à Paris, en 2016; nos interventions étaient principalement adressées à des étudiant.e.s en formation professionnelle initiale. Des formatrices et formateurs étaient cependant également présents dans la salle et, en 20I7, lorsque nous y sommes retournées, nous avons également organisé une rencontre avec elles et eux. Cela nous a permis de tester ces DVD et notre approche dans un autre contexte politique et institutionnel (autour des enjeux français de restructuration du champ professionnel de la petite enfance), tout en retrouvant des problèmes pratiques des stagiaires, en partie similaires.

Ouverture pour d'autres aventures

de construction d'un matériel pédagogique

Nous envisageons de poursuivre en proposant une offre de formation courte à destination des formatrices et formateurs qui s'appuierait sur l'usage de ces DVD, et dont les contenus seraient orientés sur une discussion au sujet des conséquences pédagogiques de leur usage, selon les modèles de professionnalité utilisés (qualification, compétence, agencement).

A la suite de cette expérience, d'autres aventures vers la construction de matériel audiovisuel ont vu le jour et sont, pour certaines, encore en cours: une plateforme web pour visibiliser le travail des modératrices et modérateurs de Noctambus et accompagner les nouvelles personnes engagées (recherche mandatée par l'Association Noctambus et financée par la Fondation Meyrinoise), ${ }^{12}$ une plateforme web de formation pour les professionnel-le.s travaillant à la Résidence de la Rive auprès de personnes atteintes de troubles cognitifs (recherche mandatée par la Fondation Butini) et une recherche avec Action Innocence visant, là encore, la fabrication d'une plateforme, pour à la fois accompagner les nouvelles personnes engagées et la transformation des pratiques actuelles d'intervention en milieu scolaire (recherche mandatée par l'association Action Innocence). 


\section{Spécificités et conditions d'une aventure singulière}

Nos projets ne bénéficient pas d'un «kit» théorique et méthodologique tout prêt à être appliqué, d'un mandat de prestation ou d'une recherche à l'autre. $\mathrm{Si}$, au fil des projets, nous avons construit et stabilisé une manière de modéliser les activités, en appui sur un cadre théorique et sur une méthodologie exprimant nos présupposés épistémologiques, d'un projet à l'autre, ce socle est enrichi et repris, en un mot transformé par les nouveaux apports spécifiques. Une démarche à chaque fois singulière se construit, avec les équipes et les directions; une démarche qui prend toujours la forme d'une aventure intégrant différentes activités et différentes dynamiques.

C'est ainsi que chaque projet impose ses effets à notre outillage conceptuel et méthodologique, en l'amenant à expérimenter l'intégration de nouvelles idées et de nouvelles manières de procéder selon les contingences de chaque projet. Dans cette transformation se jouent tout à la fois de la nouveauté (via la découverte d'un nouveau champ de pratiques ou d'un nouveau terrain, d'un nouveau questionnement d'équipe ou plus largement d'institution aux prises avec son champ de pratiques) et de la continuité, depuis des idées avec lesquelles nous avons travaillé jusqu'ici et qui ont porté leurs fruits en termes de modélisation du savoir-faire (par exemple, le développement dans cette recherche des notions d'ajustement et d'agencement à l'aide des notions de territoire et de perspective...).

Une telle aventure est rendue possible grâce à une certaine organisation dans l'équipe de recherche. Nous favorisons une logique programmatique sur plusieurs années, qui dessine des perspectives en termes de thématiques et de questionnements de recherche. Mais cette logique programmatique doit être suffisamment souple et ouverte pour, d'une part, intégrer à l'intérieur de l'équipe les intérêts d'étude des un·e.s et des autres et, d'autre part, accueillir favorablement des demandes externes qui surviennent en sus des projets déposés par l'équipe à des fonds spécialisés de subventionnement de la recherche. ${ }^{13}$ Cette projection à moyen, voire scientifique (FNS) ou l'Agence suisse pour l'encouragement de l'Innovation (Innosuisse). 
long terme exige un maillage serré des un·ess et des autres entre les projets et c'est cette participation systématique de tous et toutes, quoique à des intensités différentes d'un projet à l'autre, qui permet d'alimenter une programmatique collective qui évolue de manière cohérente et continue au fil des projets. Ce fonctionnement nécessite une certaine dynamique d'équipe pour que les projets soient portés de façon alternée et ajustée aux situations du moment, faite de souplesse dans les réponses et les engagements; programmatique qui est aussi transformée au fil des projets qui redessinent son orientation depuis ses apports.

Et surtout, ce qui nous permet tout à la fois de travailler dans la continuité en absorbant de la nouveauté est que chaque projet s'inscrit dans une perspective pragmatiste, qui nous amène à considérer l'activité comme étant à la fois située et induisant des savoir-faire locaux, mais aussi en prenant en considération la continuité de l'expérience et la circulation des idées et des connaissances dans le champ professionnel plus large. Considération qui nous fait accueillir les idées ou concepts depuis cette perspective.

Soulignons enfin des conditions de travail qui sont favorables à un tel fonctionnement. Tout d'abord, nous bénéficions d'un forfait d'heures dans la feuille de charge qui est laissé libre de tout engagement prédéfini, sans être attribué à des activités en début d'année et qui permet de répondre en cours d'année à des demandes qui ne sont pas prévisibles ou anticipables. Ensuite, le fait de déposer régulièrement des projets de recherche pour des demandes de financement au Fonds national de la recherche (FNS) s'avère essentiel pour garantir une certaine qualité ou solidité théorique et méthodologique et permet de dégager du temps pour cette réflexion. Pour finir, une confiance et une liberté accordées par notre institution pour les montages de projets de recherche et/ou de prestations nous permettent de bien faire correspondre nos budgets à nos intentions, et donc de sauvegarder un travail ajusté aux terrains.

\section{Conclusion: des recherches où l'on pense avec les professionnel.le.s sans quitter notre spécificité de chercheur·e.s}

Notre manière de faire de la recherche est à la fois fondamentale et orientée vers l'application, au sens où nous adoptons à la fois une position de chercheur·e classique, qui modélise et opère un travail théorique, et où nous 
travaillons en partenariat avec les professionnel.le.s au plus proche de ce qui leur importe, les intéresse. Les professionnel.le.s sont donc étroitement associé.e.s à la recherche à chacune de ses étapes, non pas au sens où elles et ils sont seulement consultés pour l'analyse ou la restitution des résultats, depuis un dualisme séparant les chercheur.e.s, qui pensent et expliquent, des professionnel.le.s qui agissent; ni, non plus, au sens d'une démarche de recherche appliquée visant à opérationnaliser dans des environnements particuliers des connaissances produites en amont par des recherches fondamentales; mais bien au sens d'une démarche de recherche visant à «faire connaissance» avec les professionnel.le.s (Stroumza \& Mezzena, 2016): apprendre à connaître leur milieu et la pratique qui s'y construit, et produire avec eux une connaissance au sujet de leur savoir-faire et des pratiques en travail social.

Cette façon de travailler implique, depuis nos présupposés pragmatistes, que le processus de recherche est non linéaire. Contrairement à la conception habituelle des liens entre chercheur.e.s et partenaires, l'ajustement aux situations dans le cours de la recherche est constant et n'est pas contingent. Il ne s'agit pas pour nous de produire nos résultats «dans le dos» des professionnel.le.s, puis de leur présenter nos résultats sous forme orale et écrite. Si le processus ne peut être décrit de manière linéaire, c'est parce qu'il est intégré. La survenue prolongée des chercheur.e•s sur le terrain des professionnel.le.s crée une perturbation potentiellement source de développement, au sens où elle exige des un.e.s et des autres d'enquêter pour réduire l'incertitude. La méthodologie, qui combine des traces des activités ainsi que des discours sur ces activités, ne laisse intacts ni les professionnel.le.s ni les chercheur.e.s. Mais on peut en dire autant de toutes les formes d'interaction entre elles et eux, des plus ordinaires au plus formalisées. C'est ainsi, tout au long du processus, qu'une démarche d'intéressement (Callon, I985) se réalise, qui reconfigure la situation de recherche et produit de nouvelles données pour les chercheur·e.s.

Dans ce fonctionnement, il n'y a pas de séparation entre recherche fondamentale et recherche appliquée, c'est-à-dire de catégorisation entre types de recherche qui correspondraient à des contrats types qui prédéfiniraient le déroulement de la recherche et la forme de ses résultats. D'un projet à l'autre, nous constatons un enrichissement de notre pensée et de nos contacts avec les terrains et une continuité liée au fait que chacun des projets concrétise une aventure féconde pour la suite. Chaque projet est ainsi lié au précédent tout en ouvrant au suivant, depuis une continuité 
dont le fil rouge est tissé au fil des transformations successives et mutuelles de nos «objets» et de notre «pensée».

En prenant au sérieux les intérêts des professionnel.le.s et les spécificités de leur contexte à l'intérieur même de notre démarche scientifique, et en étant attentives à toujours être guidées par notre intérêt de recherche, ces différents ajustements ou utilisations par les terrains de l'intervention sociale ne trahissent pas tant notre démarche (ou l'instrumentalisent) qu'ils ne l'enrichissent.

En tant que chercheures nous estimons ne pouvoir faire l'économie d'une réflexion sur la façon dont la recherche est agie dans et par le paysage actuel, d'autant plus lorsque l'entrée choisie est l'analyse des activités depuis la mouvance plus large des recherches en analyse du travail. Pour être à la hauteur de ces enjeux et répondre aussi à des demandes de supervision, nous menons une recherche financée par la HES-SO sur les effets de la nouvelle gestion publique auprès des hiérarchies (directions et cadres intermédiaires) et des éducatrices et éducateurs afin d'examiner également à quelles conditions la confiance peut permettre qu'elles et ils œuvrent ensemble à la sauvegarde du sens de leur métier.

\section{Références bibliographiques}

Barbier, J.-M. \& Durand, M. (Dir.). (20I7). Encyclopédie d’analyse des activités. Paris: Presses Universitaires de France.

Béguin, P. \& Cerf, M. (2009). Dynamique des savoirs, dynamique des changements. Toulouse: Octarès.

Callon, M. (I985). Eléments pour une sociologie de la traduction: la domestication des coquilles Saint-Jacques des marins pêcheurs dans la baie de Saint-Brieuc. L'Année sociologique, numéro spécial, La sociologie des Sciences et des Techniques, 36, 169-208.

Despret, V. (2009). Penser comme un rat. Versailles: Quae.

Despret, V. \& Porcher, J. (2007). Etre bête. Arles: Actes Sud.

Dewey, J. (I993 [1938]). Logique. La théorie de l'enquête. Paris: Presses Universitaires de France.

Durand, M. (2008). Un programme de recherche technologique en formation d'adultes. Education et Didactique, 2(3), 97-I 2 I.

Fornel, M. de, Ogien, A. \& Quéré, L. (Dir.). (200I). L'ethnométhodologie: une sociologie radicale. Paris: La Découverte.

Friedrich, J., Mezzena, S., Seferdjeli, L. \& Stroumza, K. (20I4). Comment une équipe éducative d'un centre de jour genevois agit au quotidien pour éviter l'exclusion des adolescents qu'elle accueille. Dans S. Voelin, M. Eser Davolio \& M. Lindenau (Dir.), Le travail social entre résistance et innovation/Soziale Arbeit zwischen Widerstand und Innovation (pp. 267-273). Genève: Editions ies. 
Gillet, A. \& Tremblay, D.-G. (20I7). Recherches partenariales et collaboratives. Rennes/Québec: Presses Universitaires de Rennes/Presses de l'Université du Québec.

Jonckheere, C. de (20I0). 83 mots pour penser l'intervention en travail social. Genève: Editions ies.

Kostulski, K. et al. (20I I). L'horizon incertain de la transformation en clinique de l'activité: une intervention dans le champ de l'éducation surveillée. Activités, 8(I), I29-I 45 .

Libois, J. (20I3). La part sensible de l'acte. Genève: Editions ies.

Lyet, P. \& Paturel, D. (2012). Pour dépasser les oppositions entre une recherche en, dans ou sur le travail social: une science-action en travail social. Pensée plurielle, 30-3I, 255-268.

Mezzena, S. (20I2). Engagement des travailleurs sociaux depuis le partenariat avec l'environnement: agencements et formation des valeurs. Forum, I3, 37-47.

Mezzena, S. (20I4). Connaissance et professionnalité dans la pratique comme territoire à équilibrer. Enquêtes et perspective dans l'activité des éducateurs [Thèse de doctorat]. Genève: Université de Genève.

Mezzena, S., Seferdjeli, L. \& Stroumza, K. (20I4). Des recherches qui prennent pour objet les savoir-faire. Dans D. Paturel (Dir.), Recherche en travail social: les recherches participatives (pp. 220-235). Nîmes: Champ social.

Mezzena, S. \& Stroumza, K. (2012a). Des idées agissantes dans l'activité: analyses d'enquêtes dans l'activité réelle d'éducateurs spécialisés. DIRE, 2. Consulté le 26.04.2018 sur http://docplayer.fr/30700072-De-la-reflexivite-du-sujet-auxenquetes-pratiques-dans-l-activite-d-educateurs-specialises.html

Mezzena, S. \& Stroumza, K. (20I2b). Des enquêtes pratiques à la connaissance dans les accomplissements: place des éducateurs, des chercheur.e.s et des théories dans une recherche en éducation spécialisée. Pensée plurielle, 30, I75-189.

Mezzena, S., Stroumza, K. \& Borel, C. (2014a). Travail d'intégration à l'œuvre dans les activités des éducateurs/trices de l'enfance de la Ville de Vernier. Revue [petite] Enfance, I I3, pp.8I-95.

Mezzena, S., Stroumza, K. \& Borel, C. (2014b). L'intégration dans les activités de la petite enfance: analyse du travail réalisé dans les institutions de la Ville de Vernier. Dans Actes du colloque organisé par le Service Petite Enfance de la Ville de Genève, décembre 2013 (pp.93-103). Consulté le 21.06.I8 sur http://www.villegeneve.ch/fileadmin/public/Departement_5/Documents_d_actualite/Actes_ du_Ioe_colloque_petite_enfance_L_education_l_affaire_de_tous_20I3.pdf

Mezzena, S., Stroumza, K., Seferdjeli, L. \& Baumgartner, P. (2013). De la réflexivité du sujet aux enquêtes pratiques dans l'activité d'éducateurs. Activités, Io(2), I93206. Consulté le 21.06.18 sur journals.openedition.org/activites/pdf/799

Ogien, A. \& Quéré, L. (2005). Le vocabulaire de la sociologie de l'action. Paris: Ellipses.

Quéré, L. (2006). L'environnement comme partenaire. Dans J.-M. Barbier \& M. Durand (Dir.), Sujets, activités, environnements. Approches transverses (pp.7-29). Paris: Presses Universitaires de France.

Ryle, G. (2005). La notion d'esprit: pour une critique des concepts mentaux. Paris: Payot \& Rivages. 
Seferdjeli, L. \& Stroumza, K. (20I I). Partenariat de recherche en hautes écoles spécialisées Santé Travail social de Suisse occidentale: engagement des acteurs et apprentissages croisés. SociologieS. Consulté le 26.04.2018 sur http://sociologies.revues.org/3622

Seferdjeli, L. \& Stroumza, K. (20I7). Pertinence et positionnement de l'analyse du travail comme théorie et méthode pour l'étude de la recherche partenariale. Dans A. Gillet \& D.-G. Tremblay, Recherches partenariales et collaboratives (pp.8I-Ioo). Rennes/Québec: Presses Universitaires de Rennes/Presses de l'Université du Québec.

Stengers, I. (1997). Cosmopolitiques VI. La vie et l'artifice: visages de l'émergence. Paris: La Découverte.

Stroumza, K. \& Mezzena, S. (2016). Un dispositif d'autoconfrontation pour «faire connaissance» avec les modérateurs des bus nocturnes genevois. Dans F. Ligozat, M. Charmillot \& A. Muller (Ed.), Le partage des savoirs dans les processus de recherche en éducation. Louvain-la-Neuve/Paris: De Boeck.

Stroumza, K., Mezzena, S., Friedrich, J. \& Seferdjeli, L. (2014). L'ajustement dans tous ses états: règles, émotions, distance et engagement dans les activités éducatives d'un centre de jour genevois. Genève: Editions ies.

Stroumza, K., Mezzena, S., Krummenacher, L. \& Reichel, N. (2017). Le savoir-faire de la modération dans les bus nocturnes genevois: quand peur et anticipation des risques riment avec convivialité. Revue SAS, 8. Consulté le 26.04.2018 sur http://www.sas-revue.org/index.php/n-conception/47-n-8/varia-n8/i i g.le. savoir-faire-de-la-moderation-quand-peur-et-anticipation-des-risques-rimentavec-convivialite-dans-les-bus-nocturnes

Suchman, L. (1987). Plans and situated action. Cambridge: Cambridge University Press. Thiery, O. \& Cerf, M. (2009). Penser la recherche participative comme une pratique. Une proposition de diagnostic. Dans P. Béguin \& M. Cerf (Dir.), Dynamique des savoirs, dynamique des changements (p.44). Toulouse: Octarès. 


\section{L'imbrication heuristique entre terrain, recherche et enseignement}

Le cas du rapport à l'argent des AS

face à des situations de surendettement

Sophie Rodari et Laurence Bachmann

\section{Introduction}

Notre contribution vise à mettre en exergue, à partir de la question du rapport à l'argent des assistantes sociales et assistants sociaux (AS) face à des situations de surendettement, la manière dont les expériences des professionnel.le.s de terrain, l'enseignement et la recherche se nourrissent mutuellement. Cet article veut ainsi souligner la richesse des apports professionnels et de recherche au développement de l'enseignement professionnel supérieur en montrant comment l'articulation entre ces trois pôles peut favoriser le renouvellement des pratiques par une évaluation continue des besoins des professionnel-le.s et des publics concernés.

Dans le domaine de l'action sociale, c'est à partir des demandes de terrain dans les années 2000 qu'ont été initiées des réformes dans l'enseignement méthodologique professionnel; enseignement portant sur les compétences et les outils nécessaires à la modélisation des interventions sociales. Ces réformes s'inscrivent dans un contexte de transformation politique des attentes en matière d'enseignement et de recherche dans les hautes écoles (Loi fédérale sur les hautes écoles spécialisées LHES, I995) et son règlement d'application (OHES, I996). Les exigences d'accréditation nécessitent dorénavant un pilotage institutionnel des activités de recherche et d'enseignement par une meilleure coordination de celles-ci dans les sites et les filières. Notre propos constitue dès lors un bilan réflexif de cette expérience de collaboration scientifique et pédagogique. Ce bilan est construit sur le déroulement chronologique des événements à partir de l'étude du rapport à l'argent des AS en cas de surendettement. 


\section{L'évolution des besoins en matière de formation dans le service social}

L'histoire de la professionnalisation du service social ${ }^{1}$ nous informe qu'en France, dès le $19^{\text {e }}$ siècle, l'examen des conditions d'existence des personnes laissées pour compte par l'industrialisation est au cœur de cette nouvelle profession. Héritier des formes classiques de la charité, le service social construit une approche rationnelle et pédagogique de la prise en charge des "pauvres", compatible avec les exigences politiques et économiques de l'ère moderne. Cette approche repose sur une analyse des situations effectuée à partir de l'exposé des difficultés de celles et ceux qui sont aidé.e.s. Des travaux socio-historiques et anthropologiques (Zelizer, 2005; Ribert, 2005; Lazuech, 20I2) ont mis en évidence, à travers le contrôle moral et social de l'argent destiné aux «pauvres", puis aux "assisté·e·s», comment s'élaborent les normes sociales et juridiques qui fondent le versement par l'Etat d'une aide matérielle aux personnes les plus fragiles au nom de la solidarité. Avec le développement de l'Etat social moderne, les idéaux de justice et d'émancipation fondent désormais l'intervention sociale. Conseiller, orienter, assurer un revenu de substitution, défendre des droits, exercer une fonction de médiation et accompagner constituent le socle de celle-ci. L'action sociale se construit ainsi en prenant mieux en considération les aspirations et les compétences des bénéficiaires dans les interventions. Les AS demeurent des généralistes formé.e.s à la relation d'aide qui soutiennent les projets de leurs usagères et usagers par leurs compétences sociales, administratives et juridiques. ${ }^{2}$

La consolidation de l'Etat social après la Seconde Guerre mondiale en Europe et le plein emploi font reculer la grande pauvreté pendant les décennies des "Trente Glorieuses". Les questions économiques et leurs effets sociaux apparaissent ainsi moins problématiques, car le système social assure, pour la grande majorité des salarié·e·s, son rôle de protection lors d'un licenciement, d'une séparation, d'un accident ou d'une maladie. De plus, les systèmes de retraite et d'invalidité instaurés à la même époque permettent une amélioration des conditions de vie des personnes âgées et/ou invalides. Cette période de prospérité économique est à l'origine de l'essor de la société de consommation. Pour démocratiser l'accès aux biens et aux services, apparaissent les supermarchés et avec eux se généralisent différentes formes de crédit à la consommation. La déconnexion 
entre l'achat et le paiement s'instaure progressivement (Chatriot, 2006; Lazarus, 2009; 20I2). L'avènement de la société de consommation fait ses premiers dommages dans les milieux ouvriers, qui entrevoient avec le crédit l'opportunité de concrétiser leurs rêves d'insertion et d'ascension sociale en accédant à l'électroménager, à la voiture, à la propriété et aux études. Quel que soit le motif de l'endettement, c'est une faute pour l'opinion publique, qui associe pauvreté, consommation excessive et comportement irresponsable selon des représentations héritées du passé. Dans ce contexte, les personnes endettées qui s'adressent aux services sociaux reçoivent une réponse intervenant lorsque les effets de l'endettement sont visibles et que leurs conditions socioéconomiques sont détériorées. Nous qualifions cette réponse de «réparatrice», dans le sens qu'elle «répare», à la demande des personnes surendettées, les dommages subis par les créancières et créanciers. Cette réponse peut devenir «coercitive» en cas de perte durable de la capacité de discernement pour protéger les personnes et leur entourage. ${ }^{3}$ Ici, l'autorité judiciaire impose une réparation aux personnes surendettées et contrôle son effectivité.

Ainsi, les réponses du service social aux préoccupations financières des personnes en difficulté et à leur endettement seront essentiellement des réponses individuelles réparatrices, complémentaires et subsidiaires

1 Nous faisons ici référence au «best-seller» de l'époque: Le visiteur du pauvre du baron Joseph-Marie de Gérando, publié chez Louis Colas à Paris en I820.

2 L'exercice des métiers du social en Suisse se fonde sur la Déclaration universelle des droits de l'homme (I948), la Convention européenne des droits de l'homme (I953), la Charte sociale européenne (I96I), les droits fondamentaux, les droits civiques et les objectifs sociaux de la Constitution fédérale (I999) et, enfin, le document «Déontologie dans le travail social, déclaration de principes» de la Fédération internationale des travailleurs sociaux (FITS, 2004).

3 «Lorsqu'une personne est empêchée, en partie ou en totalité, d'assurer elle-même la sauvegarde de ses intérêts à cause d'une déficience mentale, d'un trouble psychique (ce qui inclut les dépendances) ou d'un autre état de faiblesse affectant la condition personnelle, l'autorité de protection de l'adulte institue une curatelle» (http://www.guidesocial.ch/fr/fiche/I4I/, consulté le 23.05.I7). De fait, la question du règlement et/ou de la gestion des dettes s'effectue rarement dans le cadre d'une procédure judiciaire instituant des mesures de curatelle, qui constitue dans l'ordre juridique suisse une mesure de dernier recours. Ces mesures impliquent, pour les curatrices et curateurs, la tenue de comptes, la gestion des revenus, le contrôle des dépenses et si possible l'assainissement des dettes, ces éléments faisant l'objet d'un rapport aux autorités judiciaires compétentes. 
à d'autres formes d'aides publiques et privées. C'est avec l'apparition du chômage de longue durée et le recul consécutif des prestations sociales de l'Etat que les questions d'argent tiendront à nouveau une place déterminante dans l'activité des AS. Toutefois, l'explosion de l'endettement privé en Suisse, ces dix dernières années, révélera que les formes habituelles de prises en charge ne déploient plus tous leurs effets. En $2014,{ }^{4}$ selon une enquête conduite par l'OFS, 40\% de la population vivent dans un ménage endetté et $8 \%$ des ménages cumulent trois types de dettes. Les dettes les plus fréquentes sont les arriérés de paiement. Une part de i $8 \%$ de la population vit dans un ménage avec au moins un arriéré de paiement. Les crédits les plus courants sont le leasing pour voiture (I5,5\%) et les crédits à la consommation (10,5\%). Plusieurs constats vont alors s'imposer. La majorité des AS devraient pouvoir se spécialiser dans le domaine pour répondre à la complexité des demandes et à la spécificité des procédures juridiques et comptables qui sous-tendent une épuration partielle ou complète des dettes. Par ailleurs, trop peu de personnes peuvent avoir accès à des services spécialisés car ceux-ci n'existent ni dans toutes les communes ni dans tous les cantons. De plus, des politiques publiques sont envisagées pour coordonner les actions des AS avec les représentant.e.s des consommatrices et consommateurs, les spécialistes des poursuites, les autorités scolaires et les employeur.e.s pour prévenir l'endettement.

\section{Concevoir des formations pour prévenir les multiples effets de l'endettement}

La prise de conscience politique et sociale que l'endettement est une forme de pauvreté pernicieuse, du fait qu'elle est invisible et que ses effets économiques et sociaux se feront sentir ultérieurement, a modifié les attentes en matière de compétences professionnelles dans le service social. L'importance et la multifactorialité de l'endettement nécessitent dès lors une adaptation de la formation supérieure en service social. Les AS qui interviennent auprès de populations en grandes difficultés financières demeurent des spécialistes de la relation, mais ils et elles disposent dorénavant de connaissances juridiques et économiques pointues, pour entreprendre des négociations avec les créancières et créanciers et représenter les usagères et usagers en justice, expertises qui sortent du champ habituel de leurs compétences (Cambier et al., 2005; Rodari, 2010). 
Pour faciliter l'accès à l'emploi des étudiant.e.s en service social au terme de leur formation, les hautes écoles (HES) en travail social ont décidé de combler ces lacunes dans l'enseignement en formation Bachelor et dans la formation continue. A Genève, un enseignement modulaire articulant droit du contrat et méthodologie d'intervention du désendettement a été mis en place dès 2003. ${ }^{5}$ Aux besoins des étudiant.e.s s'est ajoutée une demande de consolidation et de développement de l'offre de formation continue pour les AS en activité. En 2006, la demande de professionnel.le·s du service social de la mise sur pied d'un CAS en gestion de dettes visait à combler une lacune institutionnelle dans les dispositifs de formation postgrade développés par les HES en Suisse romande et à doter les AS d'une meilleure reconnaissance de leur expertise par un titre spécifique. En effet, dans ce domaine, seules des formations de courte durée organisées par des institutions sociales "privées » existaient. Le premier programme de ce CAS, conduit par la HES-SO en collaboration avec l'association professionnelle Dettes Conseils Suisse (DCS), a été largement construit à partir des expériences et des lacunes perçues sur le terrain. Ces lacunes concernent la maîtrise des procédures juridiques et administratives, l'art de la négociation et des compétences pour initier et conduire des projets de prévention. Mieux informer et conseiller, voire éduquer différents groupes de la population aux prises avec la financiarisation de la vie quotidienne, ont nécessité une transformation de l'ingénierie de formation pour y répondre. Toutefois, la mise en place d'une formation ne s'est pas avérée suffisante. En effet, l'évaluation de cette première édition du CAS a démontré que l'assise scientifique des enseignements méthodologiques devait être renforcée pour accompagner l'adéquation de la formation à l'évolution des besoins des métiers de service social. Il nous fallait, dès lors, développer la recherche sur le rapport à l'argent dans des situations d'endettement.

4 Les chiffres que nous mentionnons sont extraits de l'enquête sur les revenus et les conditions de vie (SILC) conduite par l'Office fédéral des statistiques (OFS) en 20I4. Consulté le 03.02.20I7 sur https://www.bfsadmin.ch/bfs/fr/home/statistiques/situation-economique-sociale-population/revenus-consommationet-fortune/endettement.assetdetail.39949.html

5 Il s'agit du module E7 du plan d'études cadre romand, intitulé Méthodes, techniques et outils d'intervention en service social, dont la coordination est assurée par Sophie Rodari à la Hets. 


\section{Une analyse sociologique de l'intervention auprès de populations endettées}

Pour mieux répondre aux préoccupations exprimées par les milieux professionnels et pour fonder un enseignement méthodologique sur des apports scientifiques, nous avons élaboré un projet de recherche sur le rapport à l'argent des AS dans six structures accompagnant des personnes surendettées. A cela, s'ajoutent d'autres actions:

, Un échange professionnel, en 2013-20I4, avec la Haute école de travail social de Louvain en Hainault (B) a permis l'élargissement du réseau et la coordination du $n^{\circ} 37$ de la revue francophone Pensée plurielle, consacré au rapport à l'argent et aux problématiques financières (Rodari, 20I4).

- Dans le cadre des Midis de la recherche du CERES, un cycle de conférences sur les usages sociaux de l'argent a été réalisé en 201420I5. Ces conférences, ouvertes au public, s'adressent autant aux chercheur.e.s, étudiant.e.s, enseignant.e.s qu'aux professionnel.e.s et directions d'institutions sociales. Ces conférences nous ont ainsi permis un dialogue avec des chercheur.e.s confirmé.e.s suisses, étrangères et étrangers, qui a nourri nos réflexions et soutenu nos liens avec d'autres équipes de recherche.

C'est dans ce contexte que nous avons entrepris, en 20I4, une recherche sur l'argent dans des structures sociales genevoises. Le choix de s'intéresser au rapport à l'argent des AS nous est apparu comme une évidence en regard du développement foisonnant des politiques publiques de lutte contre le surendettement, des préoccupations qu'elles soulèvent et du sentiment diffus, parmi les professionnel.le.s, de ne pas disposer des outils et des techniques d'intervention utiles pour y répondre. L'originalité de notre étude a été d'investiguer les questions d'argent dans un champ où ces thématiques ont peu été théorisées en Suisse, celui du travail social, en prenant appui sur une fonction professionnelle, celle de l'AS. Si un certain nombre de travaux, dans les domaines de l'ethnologie et de la sociologie, ont été effectués sur l'allocation de crédits par des banques de la Communauté européenne (mais pas en Suisse), mettant en avant, à l'instar des travaux de Zelizer (2005), la signification sociale de l'argent (par exemple, Lazarus, 2009; Aglan et al., 20I I), peu de recherches qualitatives avaient 
été effectuées sur le rapport à l'argent dans le cadre spécifique de la relation d'accompagnement de personnes surendettées. Si plusieurs études (Fassin, 200I ; Bessin, 2005; Tabin, 2008; Ossipow, 2008) se sont intéressées aux nouvelles formes de contractualisation de l'intervention sociale, peu de travaux ont mis l'accent sur le rôle de l'argent comme support d'agentivité et de régulation des problématiques sociales. Rappelons ici que, pour bénéficier d'un accompagnement social comme d'une aide financière, les individus ne peuvent plus se référer à leur seule qualité de potentiel.le.s bénéficiaires, mais sont désormais astreints à coopérer dans le dispositif. Pour Robert Castel: «Il s'agit de passer de la consommation passive de prestations sociales à une mobilisation des bénéficiaires qui doivent participer à leur réhabilitation" (Castel, 2003, cité dans Bingen \& Lebrun, 2007: I). Les difficultés financières ne sont donc plus seulement appréhendées comme des problématiques sociales. Elles peuvent se transformer en support à l'activation des personnes par la gestion budgétaire et administrative, considérée ici comme outil pédagogique, devant faciliter leur retour à l'autonomie économique. Si cette perspective socio-éducative n'est pas nouvelle, elle était jusqu'ici employée volontairement par les AS. Son inscription dans les règlements d'application de différentes lois sociales, à partir des années 2000, en a donc transformé les buts et l'usage. De par leur fonction, les AS disposent donc d'une marge d'appréciation du bien-fondé des problématiques financières comme des capacités de mobilisation des usagères et usagers à sortir de la spirale des dettes, qui est plus visible par son institutionnalisation (Astier, 2007). Cette institutionnalisation de l'activation peut toutefois entamer la crédibilité des interventions sociales, car elle exacerbe un sentiment d'injustice parmi les usagères et usagers qui ne seraient pas reconnus dans leurs droits et alimentent des controverses sur les critères individuels d'accès aux dispositifs d'aide (Avenel, 2003). Nous avons cherché, dans notre étude, à comprendre comment est intériorisée cette norme sociale de responsabilisation des usagères et usagers et comment sont portés par les AS les processus d'activation dans le cadre d'un assainissement partiel ou total des dettes.

\section{Problématique}

Nous nous nous sommes intéressées à la double dimension du rapport à l'argent des AS, comme révélateur de rapports sociaux et comme levier de transformation et de subjectivation, dans le cadre de leur contribution 
à l'accès aux prestations. Pour légitimer leur décision d'octroi de prestations auprès des bénéficiaires et des autorités, les AS invoquent plusieurs critères: une loi de référence et son règlement d'application, un cahier des charges, une déontologie professionnelle et des usages institutionnels. Mais aussi des valeurs et des représentations personnelles face aux usages de l'argent des potentiel.le.s bénéficiaires. Ils et elles disposent donc à la fois de critères objectifs et interprétatifs pour fonder leur décision. Nous avons postulé, dès lors, que leur rapport à l'argent constitue un révélateur particulièrement privilégié pour saisir l'utilisation de leur pouvoir d'appréciation et le type spécifique de prise en charge soutenant la mobilisation des bénéficiaires.

\section{Méthodologie}

Les AS s'appuient sur la définition juridique de l'endettement pour ancrer leur action de désendettement. Pour le travail social, le terme de désendettement qualifie à la fois la démarche processuelle d'accompagnement et les procédures administratives, comptables et juridiques qui la sous-tendent. Plusieurs modalités d'intervention sont proposées aux personnes surendettées, en fonction de leur solvabilité et de leur degré d'endettement. Dans tous les cas, elles impliquent de se restreindre financièrement, pour réserver une somme d'argent mensuelle au remboursement des dettes. Du côté des AS, la décision d'attribution d'une aide financière et/ou d'un accompagnement implique une évaluation qui expose et démontre la nécessité ou non de l'octroi d'une aide (Serre, 2009). Cette évaluation engage un travail intellectuel qui mobilise des normes, des critères, des savoirs qui permettent de départager les demandes d'aide et d'établir des priorités dans l'action. Elle donne lieu à une production raisonnée, sous forme de présentation orale de situation lors de colloques ou de rapport écrit à la hiérarchie qui, en dernier ressort, décidera de l'attribution ou non de l'aide financière et/ou du suivi demandé.

Pour identifier les fondements des décisions et leur articulation aux prises en charge développées, nous avons effectué quatorze entretiens compréhensifs avec les professionnel.le.s (treize femmes et un homme); des observations in situ de colloques où les AS attribuent les situations et examinent leurs modalités d'intervention; et, enfin, des analyses de dossiers de l'année écoulée, qui ont nécessité une intervention professionnelle comportant une gestion financière. En nous inspirant de l'approche 
compréhensive (Kaufmann, I996), nous avons donné une posture particulière aux AS questionnés, celle d'être considéré.e non pas comme un «objet» de recherche, mais comme un.e "partenaire», actrice ou acteur du développement de savoirs. Le traitement des données et l'élaboration théorique s'est effectué selon la théorie fondée sur les faits ou Grounded Theory (Glaser \& Strauss, I995). Notre angle d'analyse a privilégié le va-etvient entre éléments théoriques et immersion sur le terrain pour alimenter notre questionnement et notre processus de récolte des données tout au long de notre étude.

Résultats principaux

Sur la base de notre recueil de données, nous avons analysé le rapport des AS à l'argent de leurs bénéficiaires à partir de leur fonction professionnelle et de leurs représentations du «bon usage» de l'argent (PerrinHeredia, 20I I).

\section{Structure et dimensions des accompagnements}

Nous avons mis en exergue que l'accompagnement des AS s'effectue dans le cadre d'une démarche structurée autour de quatre grandes étapes: cerner la demande; établir un bilan qui s'affinera au fil des entretiens; élaborer un plan d'intervention sur la base des informations recueillies et attestées lors des entretiens; mettre en œuvre une prise en charge négociée avec l'usagère ou l'usager. Les deux dimensions imbriquées sur lesquelles s'articulent leurs projets d'intervention sont la dimension juridicoadministrative et la dimension relationnelle.

Nous avons pu dégager que les AS rencontré.e.s mobilisent dans la dimension juridico-administrative des éléments législatifs et réglementaires et des éléments relatifs aux rapports sociaux et aux inégalités sociales. Dans leur référence au cadre, les AS rappellent que le respect des règles, des convenances, des hiérarchies est un facteur primordial d'intégration sociale. Ces convictions renvoient d'une part à leur trajectoire personnelle et professionnelle et d'autre part à la nécessaire distance, selon elles et eux, qu'exige leur intervention. A contrario, l'invocation des règles est aussi mobilisée dans un souci d'émancipation, qui se manifeste par la préservation des droits des personnes auprès des caisses d'assurance-maladie, des services des contributions, des gérances immobilières, par exemple. Les droits restaurés par les recours gagnés sont les signes tangibles de cette posture. Sur 
le plan relationnel, les AS peuvent privilégier tantôt le soutien à la compassion, comme l'a déjà montré Didier Fassin (2004), tantôt le renforcement du pouvoir d'agir (agentivité), comme l'ont, entre autres, relevé Ana PerrinHeredia (2009; 20I I ; 20I6) dans le contexte français et Ruth Lister (I997) et Beverly Skeggs (20I5) concernant la situation anglaise. «Ne pas juger, établir la confiance, faire baisser le stress, responsabiliser les personnes, valoriser leurs efforts " constituent, selon les témoignages recueillis, les principaux leviers relationnels que les AS mobilisent dans leur prise en charge. En soutenant la mobilisation des usagères et usagers dans leur gestion administrative et budgétaire, les AS espèrent encourager des apprentissages économiques nécessaires à une gestion financière équilibrée.

En fonction du poids accordé à ces dimensions dans l'activité quotidienne, les logiques d'intervention varient d'un·e AS à l'autre et d'un service à un autre. A l'instar de Jean-François Gaspar (2012), ces logiques oscillent entre pragmatisme (répondre aux demandes en appliquant la loi), militantisme (préserver et défendre les droits sociaux des citoyen·ne.s) et humanisme (écouter et valoriser les décisions prises par les usagères et usagers). En tant que processus dynamique, l'accompagnement implique des capacités d'évaluation et d'adaptation continue. Comme le résume une AS: «Vous devez toujours faire avec ce qui est présenté par la personne. On peut faire des additions, des soustractions, mais après le bilan, il faut être créatif et avancer avec elle. " Ces constats tempèrent donc une impression, souvent répandue dans la presse et dans l'opinion publique, du caractère extrêmement directif des interventions des AS.

Le budget comme outil incontournable

Pour résoudre les problèmes financiers de leurs usagères et usagers, les AS recourent, dans leur grande majorité, au budget comme outil d'intervention dans l'assainissement financier. Son attrait est lié à sa puissance symbolique, matérialisée par la traduction concrète des difficultés économiques en chiffres. Par une confrontation chiffrée à leur situation, les AS tentent de créer une prise de conscience du processus de dégradation financière. Le budget sert ainsi à soutenir les efforts des bénéficiaires pour sortir de la spirale des dettes, en visualisant au fur et à mesure la progression de leur épuration.

Par ailleurs, le budget sert d'instrument de pilotage de l'intervention. A travers la maîtrise budgétaire s'apprécient le degré d'autonomie administrative des usagères et usagers pour effectuer leurs paiements et leur sens 
des responsabilités dans l'usage de leurs ressources. Les AS espèrent, par le réinvestissement de leurs bénéficiaires dans la gestion de leur argent, développer leurs apprentissages économiques et leur regard critique en matière de crédits à la consommation. Pour ce faire, les AS recourent à des supports concrets faciles à reproduire. Par exemple, les personnes sont incitées à classer les différents types de courriers par leur contenu et leur échéance, à faire des enveloppes regroupant les paiements et à remplir un tableau hebdomadaire ou mensuel de leurs dépenses pour mieux visualiser où va l'argent.

Nos résultats ont toutefois montré les limites d'une approche budgétaire des problématiques financières. Cette approche n'est pas pertinente dans des situations où les personnes ne disposent pas des minimas sociaux leur garantissant suffisamment d'argent pour vivre, car il n'existe pas de marge de manœuvre pour appliquer ces nouveaux apprentissages. ${ }^{6} \mathrm{De}$ même, lorsque ces apprentissages sont assujettis à des contre-prestations obligatoires pour ouvrir des droits aux prestations sociales. Dans ces situations, l'argent manque et la préservation des droits sociaux précède toute démarche de résolution des problèmes financiers.

Les enjeux des techniques de développement personnel

Nos résultats montrent que nombre d'AS mobilisent des techniques de développement personnel pour tenter de donner du pouvoir d'agir aux usagères et usagers. D'abord, des techniques telles que celles préconisées par l'Approche centrée sur la solution (ACS) écourtent le temps d'écoute de la plainte des usagères et usagers ou d'analyse de leur passé pour trouver des solutions efficaces, s'accordant ainsi avec les nouvelles politiques de gestion de la pauvreté. Ainsi, ces techniques améliorent les conditions de travail des AS. De plus, elles réenchantent leur travail en leur permettant de sortir des activités administratives, considérées par certain.e.s comme ennuyeuses, pour se consacrer au travail sur la relation, jugé passionnant. Enfin, elles permettent de contrer partiellement les rapports de force dans leurs relations avec les usagères et usagers (cadrer les personnes trop exigeantes ou

6 L'article i2 de la Constitution fédérale suisse stipule que «quiconque est dans une situation de détresse et n'est pas en mesure de subvenir à son entretien a le droit d'être aidé et assisté et de recevoir les moyens indispensables pour mener une existence conforme à la dignité humaine". Consulté le I5.05.2017 sur https://www.admin.ch/opc/fr/classified-compilation/_19995395/index.html 
gagner de la crédibilité face à des hommes plus âgés qu'elles, par exemple). Ensuite, en se focalisant sur la relation, ces techniques donnent la satisfaction aux AS de pouvoir mesurer les avancées significatives de la prise en charge, contrairement à un travail qui serait effectué sur un plan collectif, visant par exemple l'amélioration de la réglementation du petit crédit ou la simplification des procédures d'octroi des aides financières.

Nous avons décelé une confusion, souvent présente chez les AS, entre l'usage des techniques et leurs finalités (préserver et défendre les droits sociaux des citoyen-ne.s, améliorer les conditions de travail des professionnel.le.s, se débarrasser de surnuméraires, répondre aux injonctions de la loi, etc.). L'usage de ces techniques sert avant tout à pacifier le climat relationnel et peut se détacher des finalités de l'intervention sociale d'assainissement, qui impliquent l'épuration totale ou partielle des dettes. Ainsi, l'usage de ces techniques se heurte au traitement des urgences dans le cadre d'une procédure d'assainissement, lorsqu'il s'agit d'éviter une expulsion du logement ou de restaurer les droits à l'assurance-maladie, par exemple. L'AS intervient ici en ne pouvant pas prendre en compte la capacité des usagères et usagers à intégrer les enjeux de ces décisions pour préserver leurs conditions d'existence. Toutefois, certain.e.s AS relient l'usage de ces techniques à une action de mobilisation vers une sortie de la spirale des dettes, en valorisant par la parole les efforts déjà consentis ainsi que le temps passé à résoudre les problèmes afin d'éviter tout renoncement à la poursuite de l'accompagnement.

\section{Renforcer le pouvoir d'agir}

Renforcer les capacités des usagères et usagers en vue de faciliter leur retour à l'autonomie économique s'est avéré un enjeu majeur. La plupart des AS de notre corpus considèrent en effet qu'il relève de leur mission professionnelle d'accompagner les usagères et usagers dans le développement de leur pouvoir d'agir. Cette posture sous-entend l'idée que toute personne, même la plus démunie, est capable d'améliorer certaines de ses habitudes et que leur transformation doit être soutenue. Dans cette approche constructiviste, l'AS soutient l'acquisition de nouvelles compétences et de nouvelles manières de penser et d'agir. Précisons toutefois qu'un petit nombre d'AS considèrent que soutenir le développement du pouvoir d'agir des usagères et usagers ne relève pas de leur mission professionnelle. Pour ces professionnel.le·s, les usagères et usagers doivent intégrer d'eux-mêmes cette nouvelle norme de l'action sociale. Ces AS 
sont dès lors confronté·e·s soit à des personnes qui ont de l'agentivité, qui assument leurs dettes et collaborent activement administrativement à leur épuration, soit à des personnes qui n'en n'ont pas et ne se mobilisent pas. Par exemple, des personnes qui supplient les AS de les aider de toute urgence tout en ne respectant pas les règles administratives et juridiques d'une procédure d'assainissement et en ne faisant pas les efforts nécessaires pour s'impliquer dans une démarche qui nécessite une diminution de leur train de vie pour épurer leurs dettes, ainsi qu'un engagement à ne pas reconstituer de nouvelles dettes pour recouvrer leur autonomie financière. Les AS décrivent souvent ces dernières comme des personnes qui ne sont pas prêtes à changer leurs habitudes financières, ce qui relève de leur choix et de leur responsabilité, et ne les revoient généralement plus.

Les AS dont la mission consiste à soutenir le pouvoir d'agir des usagères et usagers affichent trois types de motivation, non exclusives entre elles, pour expliciter ce soutien. D'abord, dans un contexte où la charge de travail des AS est de plus en plus lourde, les AS peuvent être poussé·e·s à soutenir le pouvoir d'agir des usagères et usagers afin d'alléger leurs charges professionnelles. Une usagère ou un usager qui se débrouille dans l'accomplissement de tâches administratives qui peuvent lui être déléguées (rédaction de courrier type, recherche d'informations dans les administrations, constitution de dossiers administratifs, gestion de ses factures courantes) permet à l'AS de se concentrer sur les aspects administratifs et procéduraux relevant de son expertise. Face à l'augmentation régulière des demandes d'aide, l'indépendance administrative de certain·e•s usagères et usagers constitue ainsi un gain de temps apprécié. Ensuite, les AS peuvent être motivé.e.s à insuffler le pouvoir d'agir aux usagères et usagers pour entretenir la motivation et l'autonomie progressive à la résolution des dettes, en restaurant leur estime de soi et pour conserver une maîtrise sur le processus d'aide engagé. Enfin, les AS peuvent tenter de donner du pouvoir d'agir aux usagères et usagers pour les inciter à développer un esprit critique, notamment sur la société de consommation. Acculéee·s par des difficultés financières qui ont nécessité des privations matérielles et réduit momentanément leurs perspectives d'avenir, certain·e•s usagères et usagers se sont confronté.e.s à une redéfinition de leur rapport à l'argent et de leur consommation. Ce rôle réflexif des AS s'insère dans une contestation sociale du consumérisme en faveur de nouvelles formes de régulation marchande, où les questions de justice sociale et de durabilité sont désormais centrales (Dubuisson-Quellier, 2009). 


\section{Des résultats de recherche au service des professionnel.le·s}

L'inscription de la formation en travail social au niveau académique implique un renforcement de la recherche dans le champ du service social (Rullac, 20I I ; Lyet, 20I4; Chéronnet \& Chauvière, 20I6). En dégageant les mécanismes en jeu dans la relation d'aide des AS aux usagères et usagers surendettés, notre recherche permet une prise de conscience des effets des stéréotypes sociaux et des jugements moraux associés aux problèmes d'argent. La présentation et la discussion des résultats dans les cours permettent d'améliorer le mode de compréhension du «traitement social» du surendettement, selon l'expression de Fassin (200I), pour repenser la place de l'intervention en service social dans les politiques publiques de lutte contre le surendettement.

Notre recherche a également un impact sur la méthodologie professionnelle (comment intervenir à bon escient?) en service social pour diversifier les outils d'intervention et compléter les interventions actuelles par d'autres formes de prise en charge préventives et collectives. En ce qui concerne l'outil budgétaire, nous avons pu observer que les AS rencontré.e.s surestiment l'impartialité du critère chiffré dans leur appréciation des demandes et ignorent les effets stigmatisants du processus de catégorisation des comportements économiques qui en résultent. Ceux-ci doivent donc être déconstruits dans le cadre de la formation de base et continue des AS pour éviter que le rapport aux services sociaux ne représente une "épreuve» supplémentaire à l'épreuve des difficultés financières pour les milieux populaires (Siblot, 2006) et ainsi favoriser une réflexion et une adaptation des outils budgétaires existant à la pluralité des situations vécues par les populations aidées, notamment celles confrontées à de fortes variations de revenus et à des formes de cohabitation distinctes de la famille. Par ailleurs, la confusion autour des techniques de développement personnel devrait être décortiquée dans l'enseignement méthodologique professionnel afin d'étayer les enjeux juridiques, sociaux et éthiques du «diagnostic personnalisé» (Plot, 2009) à la résolution effective de problématiques, dans un contexte socioéconomique où les inégalités sociales demeurent. En outre, un regard nuancé pourrait s'instaurer dans le cadre de la formation sur le sens et la portée pour l'action sociale du succès suscité par les formations sur ces techniques de développement personnel (Bachmann, à paraître). Ainsi, par exemple, en regard des trajectoires 
professionnelles des AS, celles-ci n'attestent pas de compétences qualifiantes supplémentaires permettant leur promotion professionnelle et salariale par leurs employeurs.

Communiquer à toutes les étapes de réalisation de notre recherche a été important à nos yeux pour nourrir notre démarche volontairement inductive. Nous avons privilégié des communications dans des colloques internationaux et locaux qui réunissent responsables de formation, scientifiques et professionnel.e.s, afin que la confrontation des points de vue sur cet objet contribue au débat sur les perspectives possibles dans le domaine de la formation tout en dynamisant notre réflexion scientifique. Les congrès de l'Association internationale pour la formation, la recherche et l'intervention sociale (AIFRIS) ont constitué des opportunités privilégiées: A Genève, en 20I I, notre communication a porté sur la construction de notre problématique et son design méthodologique; à Porto, en 2015, les dimensions et les logiques à l'œuvre dans l'accompagnement des AS ont été au centre de notre propos et à Montréal, en 20I7, nous discutions les effets des processus de catégorisation des usages de l'argent des personnes endettées.

La vulgarisation scientifique nous a également paru utile pour faire connaître nos travaux. Un des outils de communication privilégié en Suisse romande est la revue REISO et nous avons eu à cœur de présenter nos résultats sur cette plateforme, qui permet de diffuser des connaissances et aussi d'alimenter les échanges et les réflexions croisées entre professionnel.le·s et chercheur.e.s (trois articles ont été publiés au printemps 20I7). Les thèmes de nos articles concernent la pratique quotidienne des professionnel.le·s et, à leur lecture, les AS pourront transférer dans leur accompagnement nos résultats de recherche. Nous avons aussi contribué à l'actualité scientifique en proposant et en diffusant nos résultats dans des articles et des contributions d'ouvrages, mettant en évidence leurs apports à la réflexivité dans le service social. Notons ici que la valorisation des résultats de recherche dépend fortement de nos cahiers des charges, des possibilités de dégager du temps pour écrire et des contraintes de nos partenaires de diffusion, ce qui ne nous permet pas de répondre en temps et en heure aux attentes des professionnel-le.s qui souhaiteraient disposer de nos résultats dès la fin de la recherche pour les intégrer à leurs pratiques.

Il serait pertinent de prolonger la réflexion sur l'appropriation par les professionnel.le·s de ces résultats de recherche enseignés et diffusés par divers canaux (articles, colloques, restitution dans les cours et les institutions, etc.). Notre analyse montre que, de par leur formation et leur 
fonction, les AS optent pour une grille de lecture longitudinale individuelle des situations, sur laquelle ils et elles élaborent et déclinent les étapes de leur prise en charge. L'accent mis sur la spécificité des situations sociales et l'aspect relationnel dans l'acquisition des compétences professionnelles place, de fait, au second plan les compétences d'expertise permettant de rendre compte des évolutions des problématiques et de développer des pratiques plus diversifiées. En lien avec un contexte de travail plus segmenté et plus hiérarchisé, la plupart des AS se concentrent dès lors, dans leur travail, sur la qualité des prises en charge offertes aux bénéficiaires. L'agenda professionnel quotidien des AS est ainsi organisé autour des entretiens. Peu de temps demeure disponible pour analyser ces prises en charge et élaborer des réflexions collectives sur les critères d'attribution des aides financières et la définition des priorités lors des interventions entreprises, pour dépasser un traitement au cas par cas des situations et innover en matière d'accompagnement. Notre recherche a donc mis en exergue que la pratique de la majorité des AS se caractérise par l'absence d'une lecture transversale des situations des usagères et usagers, mettant en évidence les contraintes structurelles dans lesquelles leurs problématiques prennent place et les rapports sociaux et de genre qui les sous-tendent. Seul.e.s les AS qui privilégient une logique d'intervention militante se réfèrent explicitement au contexte social et économique pour expliciter les causes de l'endettement de leurs bénéficiaires et leurs priorités d'intervention. Toutefois, ces logiques sont minoritaires au sein des structures sociales actives dans le désendettement. Ce qui ne permet pas leur reprise comme politique générale d'intervention au sein d'un service. Dès lors, nous pouvons nous demander: que font les AS de nos résultats de recherche? Sélectionnent-elles ou ils certaines informations? Ces savoirs transforment-ils leurs pratiques? Ces résultats incitentils au développement d'une posture critique? Et enfin, comment ce savoir est-il perçu dans un environnement professionnel où le savoir pratique a plus de légitimité que le savoir scientifique?

\section{Conclusion: valoriser la diversité des profils au sein des HES}

Nous avons montré dans cet article l'articulation entre demandes du terrain, enseignement et recherche. Cette articulation répond à une demande institutionnelle du Rectorat pour accompagner la qualité des enseigne- 
ments dans les écoles professionnelles et leurs applications dans les terrains. L'entrée de profils académiques expose toutefois les hautes écoles à renforcer les clivages entre professeur.e.s chercheur.e.s et professeur.e.s issu.e.s du terrain. Or, il s'agit de travailler ces liens à travers l'articulation entre terrain, enseignement et recherche. Y aurait-il des structures à mettre en place au sein des hautes écoles et avec les partenaires de terrain pour soutenir financièrement et logistiquement ces collaborations? En outre, la HES-SO, désirant articuler terrain-enseignement-recherche, dessine un profil idéal de professeur.e, qui serait (en une personne) expert.e de terrain et de la recherche et de l'enseignement. Or, convaincues que ce profil idéal ne peut pas se réaliser (contraintes pédagogiques et de recherche fondamentale s'opposant aux impératifs du terrain), notre contribution met en exergue un cas concret de l'articulation entre ces profils, mais à travers la collaboration au sein du corps professoral et intermédiaire des HES. Dans notre cas, la richesse de notre processus s'est jouée par la collaboration entre une professeure, ancienne AS, disposant d'une forte expertise de terrain (connaissance des dispositifs de prise en charge romands et suisses, du réseau institutionnel existant et de la conception et de l'exercice de l'enseignement méthodologique professionnel), et une chercheure présentant un profil d' «excellence académique» avec expérience de recherche soutenue (fonds tiers), séjours dans des universités états-uniennes reconnues et publications conséquentes. Des expériences de ce type devraient pouvoir être reconduites pour faire des émules. Il importe de ne pas uniquement soutenir des volontés individuelles, mais de développer institutionnellement les constitutions d'équipes de travail mixtes dans la conception et la direction des modules d'enseignement et des recherches.

\section{Références bibliographiques}

Aglan, A., Feiertag, O. \& Marec, Y. (Dir.). (20I I). Les Français et l'argent. Entre fantasmes et réalités XIXe-XXIe siècle. Rennes: Presses universitaires de Rennes.

Astier, I. (2007). Les nouvelles règles du social. Paris: Presses Universitaires de France.

Avenel, C. (2003). La relation aux aides sociales «du point de vue» des familles bénéficiaires. Recherches et prévisions, 72, 37-52.

Bachmann, L. (2009). De l'argent à soi. Les préoccupations sociales des femmes à travers leur rapport à l'argent. Rennes: Presses universitaires de Rennes.

Bachmann, L. (à paraître). Des hommes appréciables. Transformation du genre à l'ère du développement personnel. Paris: Ithaque.

Bachmann, L., Gaberel, P.-E. \& Modak, M. (2016). Parentalité: perspectives critiques. Lausanne: Editions EESP. 
Bachmann, L. \& Rodari, S. (20I4). Analyser le travail social. Une approche intégrant l'agentivité et les rapports sociaux. Pensée plurielle. Nouveaux modes de gouvernance, 35: I2I-I32. Consulté le 25.04.20I8 sur https://www.cairn.info/ revue-pensee-plurielle-20I4-2-page-I2I.htm

Bachmann, L. \& Rodari, S. (20I7). Dettes: les motivations des assistantes sociales. REISO, Revue d'information sociale. Consulté le I9.03.2018 sur https://www.reiso. org/document/2018

Bessin, M. (2005). Le travail social est-il féminin? Dans J. Ion (Dir.), Le travail social en débat(s) (pp. I52-I69). Paris: La Découverte.

Bingen, A. \& Lebrun, M. (2007). La contractualisation des rapports sociaux à l'épreuve de la subjectivité [Communication]. Deuxième Congrès international des formateurs en travail social et des professionnels francophones de l'intervention sociale, 3-7 juillet 2007, Namur.

Cambier, E., Perler-Isaaz, F. \& Reusse, I. (2005). Le désendettement: une pratique proposée par des services spécialisés pour lutter contre un fléau socioéconomique inquiétant, le surendettement des ménages. Dossier de l'ARTIAS, septembre-octobre. Consulté le 25.04.20I8 sur https://www.artias.ch/wp-content/ uploads/2008/04/Dossier_septembre_2005.pdf

Castel, R. (2003). L'insécurité sociale. Qu'est-ce qu'être protégé? Paris: Seuil.

Chatriot, A. (2006). Protéger le consommateur contre lui-même. La régulation du crédit à la consommation. Vingtième siècle. Revue d'histoire, 3(9I): 95-IO9.

Chéronnet, H. \& Chauvière, M. (20I6). Formation et recherche dans le travail social: de nouveaux espaces de collaboration? Sociétés et jeunesses en difficulté, I6. Consulté le o9.0I.20I8 sur http://journals.openedition.org/sejed/8I44

Dubuisson-Quellier, S. (2009). La consommation engagée. Paris: Presses de Sciences Po.

Fassin, D. (2004). Des maux indicibles. Sociologie des lieux d'écoute. Paris: La Découverte.

Fassin, D., Defossez, A.-C. \& Thomas, V. (200I). Les inégalités des chances dans l'accès aux secours d'urgence. Revue française des affaires sociales, I (janvier-mars), 9I-IIO.

Gaspar, J.-F. (20I2). Tenir! Les raisons d'être des travailleurs sociaux. Paris: La Découverte.

Glaser, B. \& Strauss, A. (1995). La production de la théorie à partir des données. Les terrains de l'enquête, I, I83-195.

Kaufmann, J.-C. (1996). L'entretien compréhensif. Paris: Nathan.

Lazarus, J. (2009). L'épreuve du crédit. Sociétés contemporaines, 76, I7-4I.

Lazarus, J. (20I2). L'épreuve de l'argent: banques, banquiers, clients. Paris: Calmann-Lévy.

Lazuech, G. (2012). L'argent du quotidien. Rennes: Presses universitaires de Rennes.

Lister, R. (I997). La protection sociale individualisée: l'exemple britannique. Les Cahiers du Mage, 3/4, I I3-I 27.

Lyet, P. (20I4). La «re-cherche» par les chercheurs et les acteurs sociaux. Un renouvellement de la construction de la réalité. Dans M. Jaeger, Le travail social et la recherche [Conférence de consensus] (pp. I00-I08). Paris: Dunod.

Ossipow, L., Lambelet, A. \& Csupor, I. (2008). De l'aide à la reconnaissance. Ethnographie de l'aide sociale. Genève: Editions ies.

Perrin-Heredia, A. (2009). Les logiques sociales de l'endettement: gestion des comptes domestiques en milieux populaires. Sociétés contemporaines, 76, 95-I I 9. 
I9I | L'imbrication heuristique entre terrain, recherche et enseignement

Perrin-Heredia, A. (20I I). Faire les comptes: normes comptables, normes sociales.

Genèses, 84, 69-92.

Perrin-Heredia, A. (20I6). L'accompagnement budgétaire, un instrument ambivalent des conduites économiques domestiques. Dans S. Dubuisson-Quellier, Gouverner les conduites (pp.365-398). Paris: Presses de Sciences Po.

Plot, S. (2009). Du flambeur à la victime. Vers une problématisation consensuelle du surendettement. Sociétés contemporaines, 76, 67-93.

Ribert, E. (2005). Dire la dette à travers l'argent ou la taire à travers le don. L'argent en famille. Terrain, 45, 53-66.

Rodari, S. (20I0). Argent et travail social, formes et fonctions de l'argent dans la relation d'aide. Dans Quand les dettes enchaînent à l'aide sociale [Actes du colloque de l'Artias] (en ligne). Lausanne: Artias. Consulté le I9.03.20I8 sur www.artias.ch/wp-content/uploads/20ıo/ı2/2Actes20ı_Rodari.pdf

Rodari, S. (20I4). Usages sociaux de l'argent et problématiques sociales financières [Editorial]. Pensée plurielle, 3(37), 7-I3.

Rodari, S. (20I7). L'éducation financière des jeunes dans le champ de l'éducation sociale». Initiale F, I5, I4-I5. Consulté le I9.03.20I 8 sur https://www.foj.ch/ initialef/numero I5/

Rodari, S. (à paraître). Surendettement et désendettement. Notices du Dictionnaire suisse de politique sociale. Berne: ASPS.

Rodari, S. \& Bachmann, L. (2017a). Contre le surendettement: un art de dentelière. REISO, Revue d'information sociale. Consulté le I9.03.2018 sur https://www.reiso. org/document/I9I8

Rodari, S. \& Bachmann, L. (20I7b). Le budget, outil de contrôle ou d'émancipation? REISO, Revue d'information sociale. Consulté le I9.03.20I8 sur https://www.reiso. org/document/I635

Rullac, S. (20I I). De la scientificité du travail social. Quelles recherches pour quels savoirs? Pensée plurielle, I (26), I I I-I 28.

Skeggs, B. (20I5). Des femmes respectables. Classe et genre en milieu populaire. Traduit de l'anglais par M.-P. Pouly. Marseille: Agone.

Serre, D. (2009). Les coulisses de l'Etat social. Enquête sur les signalements d'enfant en danger. Paris: Raisons d'agir.

Siblot, Y. (2006). Faire valoir ses droits au quotidien. Les services publics dans les quartiers populaires. Paris: Presses de Sciences Po.

Tabin, J.-P., Keller, V., Hoffmann, K. \& Rodari, S. (2004). Le «tourisme social»: mythe ou réalité, l'exemple de la Suisse latine. Lausanne: Editions EESP.

Tabin, J.-P., Keller, V., Frauenfelder, A. \& Togni, C. (2008). Temps d'assistance:

le gouvernement des pauvres en Suisse romande depuis la fin du XIX ${ }^{e}$ siècle. Lausanne: Antipodes.

Zelizer, V. A. (2005). La signification sociale de l'argent. Paris: Seuil. 



\section{Prévenir la maltraitance envers les personnes vulnérables}

De la recherche à la formation

Manon Masse

\section{Introduction}

Le thème de la maltraitance survenant dans les institutions médicales et socio-éducatives est couramment médiatisé, et le public découvre alors avec consternation l'existence de tels faits. Plusieurs recherches existantes sur la prévalence de la maltraitance envers les personnes qui vivent ou travaillent dans différentes institutions socio-éducatives (Bellis, Hughes, Jones et al., 20I2; Krug et al., 2002) exposent des limites du recueil des signalements qui laissent croire à un nombre plus élevé de cas que ceux mentionnés dans la littérature. Le premier obstacle rencontré pour signaler la maltraitance se réfère à l'ambiguïté de sa définition et, de ce fait, à l'incapacité à la reconnaître. La définition du terme maltraitance reste, en effet, équivoque. Elle regroupe des actes, des attitudes variées ou encore, à l'inverse, l'absence d'actes qui, selon le contexte où ils se déploient, pourront être perçus comme bien ou mal traitants. Plusieurs termes sont employés de manière indistincte ou comme synonymes pour parler de ces actes commis à l'encontre des personnes vulnérables et leur occasionnant des souffrances. Dans les institutions, il existe différent.e.s auteur.e.s d'actes maltraitants: il peut s'agir des personnes accompagnées, des membres du personnel salarié ou encore d'individus externes à l'institution (famille, voisins, proches, bénévoles, etc.). Sans nier l'importance ou la gravité de l'ensemble de ces actes $^{1}$, nos travaux portent uniquement sur les situations où la personne

1 Aujourd'hui coexistent plusieurs définitions de la maltraitance, qui sont apparues progressivement dans la littérature spécialisée et dans les rapports de l'OMS et du Conseil de l'Europe. Dès I992, le Conseil de l'Europe identifie divers types de 
en situation de handicap se retrouve victime de maltraitance perpétrée par un.e professionnel.le l'accompagnant. Le second obstacle à la révélation de ces actes dépend des dispositifs de signalement en milieu institutionnel; ces derniers restent rares et, quand ils existent, sont peu accessibles aux populations concernées et à leurs proches. Le troisième obstacle consiste à recevoir la parole des personnes victimes, qui sont fragilisées et dépendantes de leur entourage. En plus d'une difficulté d'expression souvent présente, leur témoignage est régulièrement mis en doute; les événements révélés sont attribués à leurs incapacités ou maladies. Enfin, les professionnel.le·s et les organisations hésitent à signaler les cas de maltraitance afin de ne pas entacher leur réputation. Malgré la difficulté à détecter ces situations, les études déjà évoquées indiquent que les personnes en situation de handicap sont quatre à sept fois plus souvent victimes de maltraitance que les autres.

Ces données sont préoccupantes, d'autant plus lorsque les faits surviennent dans des milieux dont la mission est d'accompagner des personnes vulnérables en visant leur bien-être et le développement ou la préservation de leur autonomie dans différents domaines de la vie. Les faits signalés imposent de réagir, de créer et de mettre en œuvre des moyens d'intervention et de prévention visant à les éviter.

Cette contribution décrit deux recherches menées sur ce thème avec des publics distincts. La première étude cherche à mieux délimiter la représentation de ce qu'est la maltraitance en milieu institutionnel dans des lieux qui accueillent des adultes ayant une déficience intellectuelle. Elle interroge les représentations selon le point de vue des protagonistes (professionnel.le.s, parents, personnes en situation de handicap), en tant que potentiels acteurs et actrices, victimes ou témoins. Elle vise également à identifier quelles sont pour eux les actions à déployer afin d'éviter l'apparition de la maltraitance ou d'en prévenir les conséquences pour les personnes qui en sont victimes. La deuxième recherche ${ }^{2}$ présentée analyse un programme de formation sur ce thème destiné à des étudiant.e.s en dernière année du cursus Bachelor en travail social. ${ }^{3}$ Ce module de formation a pour but de questionner leurs représentations de la maltraitance en institution dans des lieux variés où exercent les travailleuses et travailleurs sociaux auprès de diverses populations (petite enfance, enfance et adolescence, adultes, personnes âgées) ainsi que de la manière de la prévenir. La recherche vérifie en cours de formation l'évolution des représentations du phénomène et les modifications dans les facteurs de risques et de protection identifiés. Elle analyse douze à dix-huit mois après la formation 
quelles sont les actions mises en œuvre par ce groupe d'étudiant.e.s lors de la fin de leur formation et au début de leur vie professionnelle en les comparant à un groupe témoin de la même volée n'ayant pas reçu cette formation.

Finalement, nous envisageons de quelle manière la formation sur ce thème devrait se développer, autant au moment du cursus de formation de base des professionnel.le.s du social et de la santé qu'en formation continue. Ces formations pourraient être diffusées aux différent.e.s protagonistes, y compris aux personnes concernées et à leurs proches.

\section{Postulats de départ}

Les représentations que nous avons d'un phénomène guident nos actions (Moscovici, I984). Selon Jean-Claude Abric (I994), la représentation sociale est un ensemble organisé et cohérent à l'intersection des processus cognitifs

maltraitance: les violences physiques; les violences psychiques et morales; les négligences actives et passives; les violences matérielles et financières (Conseil de l'Europe, 1992).

Au sujet des populations en situation de handicap, en 2003, un groupe de travail placé sous la direction d'Hilary Brown et mandaté par le Conseil de l'Europe a formulé une définition de la maltraitance spécifique à ce groupe. Cette définition privilégie le terme abus (de l'anglais abuse) et le présente comme: «Tout acte, ou omission, qui a pour effet de porter gravement atteinte, que ce soit de manière volontaire ou involontaire, aux droits fondamentaux, aux libertés civiles, à l'intégrité corporelle, à la dignité ou au bien-être général d'une personne vulnérable, y compris les relations sexuelles ou les opérations financières auxquelles elle ne consent ou ne peut consentir valablement, ou qui visent délibérément à l'exploiter" (Brown, 2003: 9).

2 Le texte concernant cette recherche est extrait de la thèse de doctorat, Quel programme de formation pour prévenir la maltraitance en milieu institutionnel? Analyse des retombées d'une formation sur les représentations de la maltraitance, ses facteurs de protection et les actions développées. Cette thèse a été soutenue par Manon Masse à l'Université Lumière Lyon 2, le 9 janvier 2014 et n'a pas été publiée à ce jour.

3 Ce module a eu lieu à la Hets de Genève de 2009 à 2017. Il s'agissait d'un module à choix parmi les modules libres proposés en dernière année de formation, intitulé «La maltraitance en institution, devenir acteur de sa prévention: la reconnaître, rompre le silence et la traiter ». Cette formation a été donnée à environ I 20 étudiant.e.s. Ce module a été interrompu après huit années car le règlement interne de la Hets de Genève spécifie le renouvellement des modules libres après huit années, quelle qu'en soit l'évaluation. 
et de la logique sociale. Elle est formée de nos croyances, de nos valeurs, de nos attitudes, de nos préjugés et de nos savoir-faire. Ce sont nos représentations qui guident nos conduites dans nos rapports avec l'autre. Les représentations sociales permettent de comprendre et d'expliquer la réalité, de définir une identité spécifique au groupe, elles guident les comportements et les pratiques, elles permettent de justifier a posteriori les prises de position et les comportements (Abric, I994). Comme le mentionne Denise Jodelet (I989), toute représentation vise à une meilleure compréhension et à une maîtrise de l'environnement social. Ce qui suggère que, en fonction de la représentation d'actes présumés maltraitants, les actrices et acteurs de terrain les signaleront ou non, pourront offrir une aide à la victime, chercheront à résoudre la situation ou resteront silencieux et n'y donneront aucune suite. Les deux recherches présentées analysent les représentations des protagonistes quant à la maltraitance.

Les modèles de prévention et de promotion de la santé ont guidé les deux démarches de recherche proposées. La notion de promotion de la santé correspond à celle présentée par l'Organisation mondiale de la santé (OMS) dans son Rapport mondial sur la violence et la santé publié en 2002. En promotion de la santé, le but visé est celui de diminuer l'apparition des conduites à risque, par exemple: la violence, le mobbing ou les abus (Papart, 2006). Nous avons été guidée par ces modèles, car ils tiennent compte d'une vision large et complexe de la maltraitance, incluant à la fois les personnes impliquées et leurs environnements proche et éloigné.

Les deux études présentées postulent que les démarches de prévention (OMS, I986) empruntent des voies multiples et complémentaires. L'action préventive pourra porter tant sur la diminution des facteurs de risque que sur l'augmentation des facteurs de protection. ${ }^{4}$ Les dispositifs passifs reposant sur des législations émanant des pouvoirs publics ou basés sur des règlements institutionnels pourront être complétés par des modalités actives de prévention qui impliquent directement la participation des actrices et acteurs concernés (Petitpierre, 2009). Ainsi, les deux recherches proposent une médiation du message de prévention qui encourage la "prise d'autonomie des personnes concernées et la recherche de leurs propres marques. La prévention consiste ici à donner aux sujets des éléments de connaissance sur certains dangers, sur certaines menaces..." (Trefois, 2003: 3I) et à les amener à se positionner face à ceux-ci. Ces démarches mettent en place des dispositifs de formation qui obligent le participant à prendre un rôle actif dans la prévention du phénomène étudié. 


\section{Une première recherche en milieu institutionnel}

La première recherche (Masse \& Petitpierre, 20 I I Petitpierre, Masse, Martini-Willemin \& Delessert, 2013) porte sur l'analyse des représentations de la maltraitance institutionnelle auprès de trois groupes d'acteurs (personnes en situation de handicap, professionnel.le.s et proches aidants) et permet de mieux circonscrire la notion de maltraitance.

L'origine de la démarche émane d'une des deux institutions partenaires qui souhaitaient former leur personnel à la prévention de la maltraitance. L'équipe de recherche a suggéré, à la place, de réaliser une rechercheaction sur ce thème. Le premier défi quant à sa réalisation était de trouver la manière d'aborder le sujet sans menacer les institutions et les personnes concernées. Le thème de la maltraitance menace l'identité des accompagnant.e.s, il peut nuire à la réputation des institutions partenaires. Les personnes accompagnées et leurs proches pourraient perdre la confiance des membres du personnel de l'institution en percevant leur environnement comme nocif et menaçant. Afin d'éviter ces réactions et pour ne pas nuire aux protagonistes, nous avons opté pour la création préalable d'un matériel didactique, en proposant des vignettes fictives et emblématiques à analyser sous forme de scénettes vidéo (Masse, Petitpierre, Vidon \& Jossevel, 2009). Ce matériel a soutenu la réflexion des groupes de participant.e.s durant les trois moments clés de l'étude, qui ont été: I) l'identification des critères qui permettent de décrire la maltraitance; 2) le repérage des facteurs de risque et de protection, respectivement susceptibles de précipiter ou de déjouer la survenue de la maltraitance; et finalement 3) la recherche de mesures concrètes visant à prévenir l'apparition de phénomènes de maltraitance (Masse \& Petitpierre, 20I I).

Les scénettes ont été fabriquées en intégrant les notions et concepts de la maltraitance retrouvés dans la littérature (Brown, 2003; Conseil de l'Europe, I992; Corbet, 2000; Convention internationale des droits de l'enfant, ONU, I989; Rapport mondial sur la violence et la santé, OMS, 2002; Tomkiewicz \& Vivet, 2002). En résumé, ces définitions proposent une vision

Les facteurs de risque correspondent aux éléments quiprédisposent à la survenue d'une situation alors que les facteurs de protection sont ceux qui l'empêchent. 
qui intègre les différents critères à considérer pour définir la maltraitance en milieu institutionnel en décrivant des conditions environnementales, des actes, des comportements ou des attitudes, uniques ou répétés, produits de façon volontaire ou involontaire, existants ou manquants dans le contexte de vie institutionnel ou dans la relation entre une personne accompagnée et son accompagnant.e. Ces actes ou conditions de l'environnement portent atteinte à la liberté, aux droits fondamentaux, à la dignité et causent de la détresse ou un préjudice au développement et à la personnalité d'individus ou de groupes plus ou moins vulnérables, ayant ou non la capacité de discernement.

Ces scénettes ont été retenues pour leur ambiguïté quant aux différents critères présents, qui permettent de reconnaître ou non la maltraitance dans ce qui est présenté. Leur visionnement et leur analyse en groupe a justement permis d'identifier, dans les discours des participant.e.s à la recherche, les critères qu'ils utilisent pour définir la scène observée et s'ils la jugent ou non maltraitante. Trois à quatre personnages interviennent dans chaque vignette. Ils correspondent aux actrices et acteurs impliqué.e.s dans une situation d'abus (victime potentielle d'un acte de maltraitance, auteur.e potentiel.le de l'abus, témoin direct ou indirect). La présence d'un témoin a été jugée importante car celui-ci joue souvent un rôle déterminant dans la suite qui est donnée à une situation d'abus. Pour les tester, ces vignettes ont été visionnées par un public similaire à celui engagé dans la recherche (personnes en situation de handicap, familles, professionnel-le·s). Pour environ la moitié d'entre eux, les scénettes présentées relevaient de maltraitance; pour les autres, elles consistaient en des scènes banales de la vie quotidienne en institution. Après avoir testé ce matériel vidéo-théâtre, nous avons retenu deux vignettes présentant deux thématiques, celle de l'irrespect de l'intimité et celle de l'irrespect du rythme d'autrui avec l'imposition de participer à une activité.

Le déroulement de la recherche a bénéficié de la collaboration de deux grandes institutions spécialisées dans l'accueil en hébergement et l'accompagnement en atelier de travail de personnes ayant une déficience intellectuelle. Ces établissements sont respectivement situés dans les cantons de Vaud et de Genève. En plus des membres de la direction de ces établissements, cinquante-huit personnes ont participé à cette recherche, parmi lesquelles dix-sept personnes en situation de handicap, dix-huit parents et vingt-trois professionnel-le.s. 


\section{Retour sur la méthodologie}

La démarche consiste en une élaboration collective avec les différents partenaires. Ce travail porte d'abord sur les représentations puis sur les concepts afin de dégager avec les participant.e.s, la définition de ce qu'est la maltraitance en milieu institutionnel. A partir de deux courtes situations fictives présentées sous forme de vidéo, le rôle de la chercheure a été de faciliter l'émergence des représentations et d'en faire une restitution aux participant·e·s en introduisant les choix théoriques qui ont guidé l'analyse.

La chercheure recevait la parole du participant, recueillait les données en les transcrivant, les organisait puis les restituait au groupe la séance suivante, en décrivant sa méthode et ses choix théoriques; dans ce cas-ci, le modèle écosystémique de développement de Bronfenbrenner (1979; 1996) ${ }^{5}$ et les concepts de prévention (OMS, 2002; Papart, 2006). (Masse \& Petitpierre, 20I I : 208)

Après un travail de douze mois en sous-groupes distincts, la suite du dispositif méthodologique a consisté à deux reprises en une mise en commun des propos des différents groupes avec débats et échanges. Ces confrontations croisées en direct entre les participant.e.s des différents groupes d'une même institution, puis entre les deux institutions ont enrichi les réflexions et le partage des représentations et les savoirs des un.e.s et des autres. Le climat de confiance a certainement été maintenu par le fait que les membres des groupes ont accepté de présenter eux-mêmes la synthèse des réflexions menées dans leur groupe aux participant.e·s des autres groupes.

Les participant.e-s ont pu constater des préoccupations et propositions similaires émanant des différents groupes. De leur côté, les personnes en situation de handicap ont été plus promptes à identifier l'inacceptable dans les attitudes et conduites de certains protagonistes des scénettes proposées; elles ont largement contribué à une réflexion et au questionnement sur les pratiques (Masse \& Petitpierre, 20I I : 2I I).

5 Le modèle écosystémique (Bronfenbrenner, 1979; 1996) postule une relation entre l'individu et ses environnements proches et éloignés selon différents sous-systèmes imbriqués les uns dans les autres. Il situe la personne au centre d'un cercle autour duquel gravitent différents sous-systèmes en interrelations les uns aux autres. Ce modèle a été utilisé notamment pour analyser les phénomènes de la maltraitance selon les différents sous-systèmes en présence (Belsky, I993). 


\section{Retour sur les résultats}

L'identification de ce qu'est la maltraitance est une étape capitale à sa prévention. Tout comme ce qui se retrouve dans la littérature actuelle (Brown, 2002; Belsky, I993; Corbet, 2000; ONU, 2002), les participant.e.s impliqué.e.s dans la démarche retiennent une définition de la maltraitance plutôt large comprenant différents critères (description de l'acte, contexte, fréquence, intensité, conséquence pour la victime, intention de celui qui commet l'acte). La maltraitance est considérée à la fois dans ses dimensions physiques et psychologique, et peut aussi découler d'une absence d'actes.

Certaines conduites de l'accompagnement éducatif ont été considérées comme inadéquates, non pas en se référant à des critères absolus, mais plutôt en les mettant en relation avec le niveau d'autonomie perçu de la personne à laquelle elles étaient adressées. Le manque de planification des actes socio-éducatifs ou, à l'inverse, l'excès de sollicitations dans certains autres cas ont tous deux suscité des réactions négatives, ce qui laisse penser que la perception de la qualité du suivi réside dans l'état d'équilibre et d'ajustement nécessaire à l'action socio-éducative (Masse \& Petitpierre, 20I I : 2I 2).

Les participant.e·s se sont montré.e·s sensibles face à certains manquements tels que l'irrespect envers la personne (parole, geste, ton de la voix) ou l'omission de certains gestes qui semblait caractériser l'absence de réponse à des besoins fondamentaux (manque d'écoute ou de suivi d'une demande). Toutefois, ils ne faisaient pas du tout appel à la notion de négligence pour qualifier les conduites qui s'expriment par omission (Masse \& Petitpierre, 20I I).

Le terme «maltraitance» se rapporte à des critères multiples conjugués ou non, ainsi qu'à des seuils de tolérances distincts à ces mêmes critères. C'est à partir de l'identification des variables déjà mentionnées telles que: l'acte commis (tirer le bras, injurier, ne pas agir, faire intrusion dans la pièce), le contexte, (se trouver dans une situation d'urgence, devoir gérer les demandes d'une personne tout en prenant en compte celle du groupe, par exemple), son intensité et sa fréquence, ses conséquences pour la personne, l'intention de celui qui le commet, en complément du seuil perçu, que chacun juge un acte maltraitant ou non (Masse \& Petitpierre, 20I I : 2I3).

Les facteurs identifiés et les seuils tolérés pour chacun d'entre eux reposent sur les expériences vécues par chacun·e. Les résultats des analyses ne 
démontrent toutefois pas de différence significative entre les groupes des proches aidant.e.s et ceux des professionnel.le·s. (Masse \& Petitpierre, 20I I).

Dans l'institution vaudoise, les mesures de signalement mises en place étaient connues tout autant du groupe des professionnels et des personnes handicapées que des parents. Celles-ci consistent en des mesures internes (Monsieur et Madame SOS et la commission maltraitance) et externes (signalement des mesures de contrainte à un organisme étatique extérieur à l'institution). Ces dispositifs sont accessibles à la plupart des partenaires et sont utilisés régulièrement. De plus, les mesures mises en place au niveau cantonal ont sans aucun doute favorisé une visibilité des situations d'abus commises en institution, plus particulièrement celles concernant des mesures d'isolement ou de contention. Le secret contenu dans l'univers clos de la sphère institutionnelle a pu être rompu.

Dans l'institution genevoise, il n'existait aucun dispositif externe de signalement, mais plusieurs mesures internes étaient établies. Ces mesures de signalement étaient cependant inconnues des participant.e.s en situation de handicap. Les professionnel-le.s ont réussi, en groupe, à les identifier progressivement. Quant aux familles, elles préfèrent se référer au personnel cadre ou aux éducatrices et éducateurs de référence en cas de difficulté et trouvent là une source d'information en général satisfaisante; elles n'ont pas spontanément énuméré les mesures de signalement existantes. Dans cette institution, apparaît un manque d'accessibilité des mesures de signalement pour les personnes en situation de handicap puisque le système en place propose des mesures écrites qui exigent le passage par un tiers (Masse \& Petitpierre, 20 I I).

Les facteurs de risque et de protection principalement identifiés ont été, par exemple, la difficulté de se déplacer ou de s'exprimer verbalement pour les personnes ayant une déficience intellectuelle ou le manque de formation du personnel accompagnant. Les facteurs mentionnés se réfèrent tout autant aux conditions de l'environnement institutionnel (par exemple, des horaires hebdomadaires continus trop chargés ou une surcharge de tâches organisationnelles). Finalement, la démarche de recherche a abouti à la formulation d'un répertoire d'actions préventives, élaboré par les participantes et les participants. Parmi les actions préconisées figurent, en tête, la formation au moment de la formation initiale et le développement de groupes de parole pour les personnes vivant ou travaillant dans ces institutions. C'est de ces recommandations qu'est née l'idée du deuxième projet de recherche que nous vous présentons ci-dessous. 


\section{Une deuxième recherche axée sur la formation}

Le deuxième projet de recherche (Masse, 20I4) a été mené dans le cadre d'une recherche doctorale. Il analyse un programme de formation à la prévention de la maltraitance et ses retombées. Ce programme a été élaboré dans une perspective de prévention active, en tenant compte de l'évolution du concept de maltraitance, de la nécessité d'un travail préalable sur les représentations et d'une vision écosystémique de cette problématique.

Le programme de formation élaboré propose trois modalités pédagogiques distinctes (pédagogie interactive, pédagogie transmissive et pédagogie par projet) afin d'en vérifier les retombées. Son contenu repose pour une grande part sur les recommandations émises lors de la première recherche. Ce module de formation a été dispensé à une quinzaine de travailleuses et travailleurs sociaux en dernière année d'une formation initiale pendant huit années, de 2009 à 2017.

La démarche de recherche s'est déroulée lors de la première formation (2009-20I0). Ce module représente cinq crédits ECTS et est équivalent à I50 heures de travail étudiant.e, réparties sur une durée de huit mois. La recherche vérifie l'hypothèse que des changements de représentations surviennent sur ce qu'est la maltraitance et ses facteurs de protection en cours de formation et que ces derniers pourront amener le déploiement d'actions spécifiques afin de la prévenir. Elle permet d'une part de dessiner des perspectives méthodologiques pour l'analyse d'une telle formation et d'autre part de faire des recommandations quant aux contenus et aux modalités pédagogiques à prioriser.

\section{Le programme de formation}

Le dispositif de cette recherche inclut la création d'un programme pédagogique. Comme nous étions confrontée à l'absence d'un programme déjà établi et à la rareté du matériel pédagogique sur le thème de la prévention de la maltraitance en milieu institutionnel, nous avons dû créer le dispositif de formation et le matériel pédagogique à proposer aux étudiant.e.s. Dans le cursus Bachelor en travail social, il existait des cours sur la maltraitance survenant au sein des familles, ou encore sur les situations de violence incluant celles des usagères et usagers. En revanche, au moment de mettre en place cette formation, il n'existait rien sur la maltraitance 
des professionnel-le·s à l'égard des personnes accompagnées; il en est de même dans les formations de base dans le domaine de la santé.

C'est à partir des résultats de la première recherche et des rares documents et textes existant sur des formations continues courtes et des sensibilisations offertes tant aux personnes en situation de handicap qu'au personnel accompagnant au sein des institutions socio-éducatives et de la santé, que, avec une enseignante et un enseignant, nous avons conçu le programme de ce module. Nous avons retenu plusieurs dimensions pour la création d'une formation d'une durée de dix jours, qui sont: les représentations de la maltraitance et ses facteurs de risque et de protection; ses dimensions socio-politiques, éthiques, juridiques ainsi que les dispositifs de signalement et d'aide aux victimes. Pour terminer, afin de rendre les participant.e.s davantage actrices et acteurs du processus, nous leur avons demandé de concevoir eux-mêmes une formation fictive pour prévenir la maltraitance en milieu institutionnel, qu'ils devaient présenter à des groupes de pairs travailleuses et travailleurs sociaux. Afin de les accompagner dans la démarche d'élaboration d'une formation, nous avons rajouté des contenus relatifs à la "formation des adultes "6 qui ont été insérés entre les contenus relatifs au thème de la prévention de la maltraitance.

Au niveau pédagogique, le programme du module s'inspire largement de la mise en activité en groupe et en individuel, par la résolution de situations complexes, analysées puis jouées dans sa première partie; par le choix d'un sujet personnel et sa problématisation dans sa deuxième partie; enfin, par le défi de créer un projet de formation fictif dans sa troisième partie. La participante ou le participant est engagé.e tout au long du module dans un processus qui vise à transformer ses représentations par des mises à l'épreuve tant personnelles que collectives.

En outre, le programme de formation élaboré et analysé dans le cadre de cette recherche a été conçu afin de permettre aux participant·e.s à la démarche de constituer une communauté d'apprentissage dans une perspective socio-constructiviste. Cette communauté «intègre un ensemble d'approches et de stratégies pédagogiques spécifiques complémentaires dans les activités d'un groupe qui s'associe autour d'objectifs communs cours des travailleuses et travailleurs sociaux, nous souhaitions mieux outiller les participantes et les participants afin qu'ils puissent concevoir une formation. 
d'apprentissage et qui favorise une dynamique de dialogue pour apprendre ensemble, les uns des autres, les uns avec les autres»(Orellana, 2006: 70). Par ce moyen, l'étudiant.e confronte ses idées avec ses pairs du même groupe. Il est envisagé que l'expérience de cette communauté d'apprentissage l'oriente à s'intégrer dans des communautés de pratique, une fois ses études terminées.

Le module de formation a été conçu avec pour objectif son analyse dans le cadre d'une recherche doctorale. Sa conception repose, au niveau des fondements théoriques, sur le triangle pédagogique de Jean Houssaye (2000), ${ }^{7}$ complété des apports de Richard Faerber (2002). Faerber conçoit un tétraèdre et fait du groupe un nouveau pôle en y rajoutant une dimension de coapprentissage entre pairs. Ce pôle permet de mettre l'accent sur les interactions entre individus qui pourront contribuer à l'acquisition des connaissances. Ce modèle correspond à ce que nous avons observé lors du module de formation. Il met en évidence des interactions permettant l'apprentissage par la participation, le partage et la facilitation qui émane de l'expérimentation en situation, ce qui a pu être fait par les étudiant.e.s au moment de la mise en place de leur projet de formation.

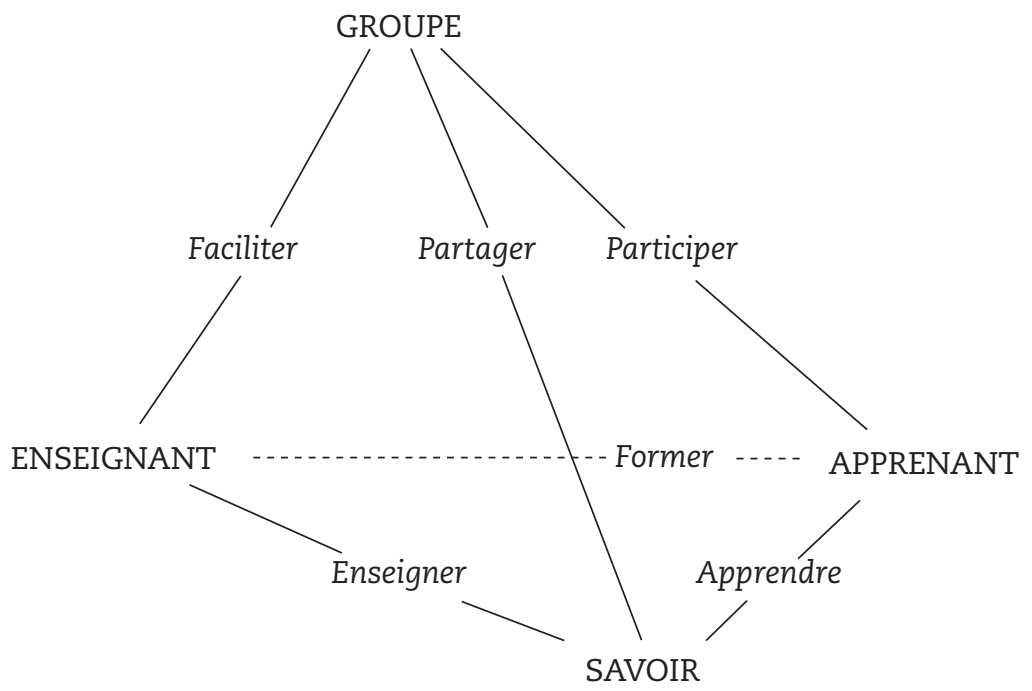

Figure I: Tétraèdre pédagogique de Richard Faerber (2002) 
Pour le choix de l'ordre des modalités pédagogiques proposées, comme nous souhaitions vérifier l'évolution des représentations sur la maltraitance et ses facteurs de protection en fonction des modalités d'apprentissage et contenus apportés, nous avons spontanément opté pour que la première modalité soit celle du travail sur les représentations de la maltraitance; la deuxième partie se concentrant sur les apports théoriques et la troisième sur l'élaboration d'un projet de formation fictif, présenté ensuite à un public de pairs.

Cette séquence d'apprentissage nous est apparue comme celle qui respectait à la fois l'importance d'un travail sur les représentations a priori des étudiant.e.s et les exigences d'une formation préventive éducative et non persuasive, afin de permettre d'impliquer progressivement l'étudiant.e à sa démarche de formation pour le rendre de plus en plus actrice ou acteur de la prévention à la maltraitance en milieu institutionnel.

\section{Retour sur la méthodologie}

Cette recherche développe une méthodologie qualitative doublée d'un plan de recherche à mesures répétées. Comme le relatent Thierry Karsenti et Lorraine Savoie-Zajc, plusieurs chercheures et chercheurs dans le domaine éducatif sont tenant.e.s des méthodes qualitatives et postulent que:

7 Le modèle du triangle pédagogique de Jean Houssaye a aidé à entrevoir comment organiser les modalités pédagogiques proposées aux étudiant.e.s dans le module de formation. Houssaye (2000) représente les situations d'apprentissage par un triangle pédagogique. Il y décrit trois pôles représentés par les trois angles d'un triangle isocèle où se retrouvent l'apprenant·e, l'enseignant·e et le savoir. Ces pôles sont eux-mêmes entourés d'un contexte qu'il nomme l'institution. Pour Jean Houssaye (2000), les situations pédagogiques ont tendance à associer deux des pôles pendant que le troisième devient inexistant, il «fait le mort». Selon lui, dans un programme pédagogique, il importe de laisser une place aux diverses associations possibles. Lorsque la transmission de savoir se fait par l'enseignant·e, l'étudiant·e «fait le mort», c'est-à-dire qu'il écoute, c'est l'action «enseigner» qui domine. Quand l'enseignant.e crée une relation particulière avec l'apprenant.e sans chercher à lui imposer son savoir, mais plutôt en accordant une grande place à leur relation, c'est alors le pôle "former" qui domine et, finalement, quand l'apprenant.e cherche par lui-même à s'approprier le savoir, c'est le pôle «apprendre » qui devient prédominant; dans ce troisième cas, c'est l'enseignant.e qui «fait le mort». 
[...] le monde social est significativement différent du monde naturel, que nous ne pouvons pas le concevoir par nos sens, mais plutôt par un travail d'interprétation, que l'expérience humaine se caractérise par la continuité, mais aussi par le changement et qu'en raison de cela, elle est imprévisible, que les problèmes humains sont locaux, toujours contextuels et, par conséquent, que les sciences sociales et humaines ne peuvent pas prétendre à l'élaboration de lois générales (Karsenti \& Savoie-Zajc, 2004: 28).

Cette démarche analyse un processus d'apprentissage par l'observation en situation des acteurs en présence et de leurs interactions; elle décrit de façon détaillée les modalités pédagogiques mises en œuvre et les contenus enseignés. Son but est de mieux comprendre ce qui se joue au moment d'une situation d'apprentissage sur la thématique de la prévention de la maltraitance, puis d'en vérifier les retombées du point de vue des changements de représentations chez les étudiant.e.s pour en explorer les applications concrètes qui découlent de cet apprentissage.

Il s'agit d'une démarche participative, qui implique les sujets dans l'élaboration et l'approfondissement de la thématique étudiée. La posture du ou de la chercheur.e en est une de proximité, sans implication directe. Dans cette démarche, les étudiant.e.s sont invité.e.s à analyser leurs représentations et le processus de formation qui leur a été proposé. De leur côté, les enseignantes et enseignants analysent la formation qu'ils ont donnée et ils collaborent à sa conception de différentes manières.

Le recueil de données s'est fait par observations simples, par questionnaires individuels répétés administrés avant, au moment de la formation et après celle-ci, par entretiens collectifs durant la formation, par des entretiens individuels avant et après la formation. Les personnes interrogées ont été les participant.e.s ainsi que les enseignant.e.s impliqué.e.s dans la démarche.

La démarche nous semble a posteriori bien adaptée à l'analyse des retombées d'un programme de formation. Complexe et exigeante à appliquer en termes d'ajustement au programme de formation et de temps alloué, elle a toutefois permis de bien décrire le contexte, la formation, ses participant.e.s et de vérifier nos hypothèses.

Enfin, les recueils de données ont été perçus positivement par les étudiant.e.s. Ils ont été bien intégrés au fil du module sans perturber le déroulement des enseignements. La plupart des étudiant.e.s ont participé à toutes les étapes et aux différents recueils de données sur une longue durée, car 
la démarche faisait sens pour eux et contribuait à l'approfondissement de leur réflexion sur la thématique.

Ainsi, cette démarche qualitative, sans se définir comme une rechercheaction, «cherche à mieux comprendre pour ensuite agir... [elle] poursuit souvent des buts pragmatiques et utilitaires, c'est-à-dire qui peuvent déboucher sur des applications pratiques des résultats obtenus" (Karsenti \& SavoieZajc, 2004: I 25). Cependant, étant donné l'engagement des étudiant.e.s au processus et des similitudes avec certaines dimensions d'une rechercheaction, nous y avons appliqué des recommandations propres à la réalisation de ce type de démarche. La recherche-action exige du ou de la chercheur.e, au moment de l'élaboration de son projet, de considérer l'ensemble des éléments du système en présence et de définir au préalable les rôles de chacun·e, la transformation visée, l'environnement dans lequel s'inscrit la recherche ainsi que la vision du monde du ou de la chercheur·e impliqué·e (Dolbec \& Clément, 2004).

\section{Retour sur les résultats}

Nos premières hypothèses ont été confirmées. Dans la plupart des cas, il existe des changements de représentations sur la maltraitance et ses facteurs de protection en cours de formation. Par exemple, les personnes qui expliquaient la maltraitance par des mauvaises conditions de l'environnement institutionnel (problème de dotation en personnel, horaire inadapté, mauvaise gestion, etc.) prennent davantage en considération les dimensions reliées aux relations interpersonnelles entre les protagonistes; les participant.e.s qui attribuaient la maltraitance à des conflits interpersonnels l'analysent de manière plus complexe en considérant également le contexte et ses contraintes, les caractéristiques des protagonistes, etc.

L'analyse documentaire réalisée préalablement à notre partie empirique nous avait permis de constater que, au cours des trente dernières années, des changements étaient survenus au niveau des représentations de la maltraitance quant aux critères retenus pour la définir. Cette évolution apparaît par une inclusion tardive des formes passives de maltraitance (négligence, abus psychologique) et par celles qui sont commises de façon involontaire, sans intention de nuire à la personne concernée. Lorsque ces formes d'abus portent préjudice aux personnes qui les subissent, elles ont été progressivement rajoutées dans plusieurs définitions. Ainsi, dans les dernières définitions de la maltraitance, la mention des conséquences des 
actes commis sur la victime devient plus importante que la nature de l'acte ou que l'intention, malveillante ou non, des actes commis.

Les résultats de la recherche montrent qu'à propos des changements de définition de la maltraitance, les effets de la formation sont variables selon les sujets. Cependant, tout comme ce qui a été observé dans la littérature, les critères les plus fréquemment rajoutés à la définition de ce qu'est la maltraitance chez les participant.e.s concernent la reconnaissance d'actes non intentionnels et les conséquences de ceux-ci pour la victime. Le rajout de ce dernier critère montre une attention plus grande portée au vécu de la victime, qui permet de questionner certains fonctionnements institutionnels. Selon les participantes et les participants, l'intrusion dans l'intimité et les abus de pouvoir seraient des actes fréquemment rencontrés en milieu institutionnel.

De surcroît, en élargissant le panel des actes initialement considérés comme maltraitants, en y incluant la négligence et des abus psychologiques tels que l'irrespect de la personne, de son intimité, ou encore l'abus de pouvoir ou l'ignorance face à des demandes formulées survenant dans la vie quotidienne, les participant.e.s ont relevé la manifestation très fréquente d'actes de maltraitance au sein des institutions où ils avaient travaillé ou réalisé des formations pratiques. Il en surviendrait chaque semaine, voire chaque jour, selon certain.e.s. Aux dires des étudiant.e.s, cette maltraitance semble se développer progressivement, en commençant précisément par des actes d'apparence anodine qui sont tolérés. Afin de la prévenir et d'en éviter l'escalade, il nous semble primordial de ne pas hiérarchiser les abus commis envers les résidentes et résidents, envers les travailleuses et travailleurs de ces lieux d'accueil, à partir d'un critère pris isolément. En effet, concernant la nature des actes maltraitants, il paraît évident que les abus sexuels et physiques sont plus rapidement identifiés par les participant.e.s à la formation car ils sont particulièrement traumatiques et touchent le corps propre et l'intégrité de la personne. Il n'en reste pas moins que les souffrances et séquelles dues à la négligence (ignorance, oubli) ou les abus psychologiques (paroles dégradantes, injures, mépris) peuvent entraîner des conséquences graves pour les victimes lorsqu'ils se produisent souvent et se perpétuent. Lors d'un exercice d'analyse des conséquences de l'irrespect de la sphère privée ou du rythme de la personne, et ce de façon répétée, les étudiant.e.s ont identifié un risque accru pour la personne de manque de confiance et d'estime de soi, de dépression, d'auto-agression ou d'hétéro-agression, de diminution des échanges 
sociaux, de renfermement sur soi, de manque d'appétit, de négligence de son apparence et de son hygiène, etc. Pourtant, au premier abord, les comportements visionnés étaient décrits comme très routiniers en milieu institutionnel. Ainsi, c'est dans l'agencement de ces différents critères (forme, durée, contexte, répétition, intention) que la gravité d'un acte pourra être mesurée, et cela en tenant compte des conséquences exprimées par la victime lorsqu'elle a la possibilité de le faire. Seule une analyse approfondie permettra d'évaluer l'importance de l'abus et il nous semble arbitraire d'établir une classification en négligeant cette étape.

Les facteurs de protection identifiés sont multiples et se répartissent dans l'ensemble des sous-systèmes du modèle écosystémique (Bronfenbrenner, I979; Belsky, I993); ils peuvent être cumulatifs ou interférer les uns avec les autres.

L'analyse de l'évolution de l'identification des facteurs de protection au cours de la formation permet de confirmer l'hypothèse de leur modification. Les participant.e.s ajoutent des facteurs de protection appartenant à différents niveaux. Par exemple, l'analyse a permis de dégager, des questionnaires et entretiens des étudiant.e.s, des actions possibles qui visent l'organisation, la formation, le soutien des personnes, l'expression, l'information, l'amélioration des dispositifs de signalement et, de façon moindre, l'amélioration des procédures, des lois et des règlements ainsi que le développement de systèmes de contrôle. La plupart des mesures à actualiser viseraient directement le ou la professionnel-le, puis la personne en situation de handicap et, de manière moins prononcée, les parents, la direction de l'établissement et la société.

Au sujet des actions concrètes réalisées douze à dix-huit mois après la formation, en comparaison avec le groupe témoin de la même volée, nous avons constaté que plusieurs actions avaient été mises en route par les étudiant.e.s en cours de formation ou dans les douze mois après l'avoir suivie, qu'il s'agisse d'un approfondissement à la fin des modules de leur formation initiale ou lors d'une formation continue ou d'actions directes dans leur pratique. Même si ces dernières restent limitées, les retombées portent sur la réflexion personnelle, une plus grande facilité à échanger avec les pairs et la direction sur ce thème ainsi que sur une augmentation de la vigilance face à des actes maltraitants commis par autrui ou par soi-même dans la pratique. De plus, les étudiant.e.s ayant suivi cette formation abordent le thème de la maltraitance lors de supervisions individuelles ou collectives. En comparaison, les étudiant·ess du groupe témoin 
n'avaient pratiquement pas, à ce moment-là, accompli d'actions, ni en termes d'approfondissement de cette thématique en fin de formation ou en formation continue ni par des réalisations dans leur pratique de terrain.

Cette recherche nous permet de suggérer certains contenus qui, au regard des résultats, nous paraissent essentiels à traiter dans une formation sur cette thématique. Il s'agit des dimensions éthiques et déontologiques (valeurs, responsabilité, pouvoir dans la relation à l'autre, communication dans le travail d'équipe), des dimensions socio-politiques et culturelles ainsi que des dimensions légales et des dispositifs de prévention institutionnels. En outre, la difficulté d'appréhender les conséquences d'un acte pour la victime afin de le décrire comme maltraitant nous a amenés dans les années suivantes de la formation à rajouter des contenus théoriques sur les indices cliniques permettant de détecter la maltraitance. Dans le même sens, des apports sur la manière de recevoir les témoignages des victimes et le rôle des instances internes et externes à l'institution ont été rajoutés. Le but étant de mettre davantage l'accent sur le vécu de la victime au moment de l'enclenchement d'une démarche de signalement. Ces nouveaux contenus laissent voir aux futur.e.s professionnel.le.s de terrain l'importance de développer des stratégies d'action visant au développement des compétences des personnes accompagnées afin de leur permettre de se protéger du risque de maltraitance. Ces compétences comprendraient notamment l'accès à des formations permettant de développer, entre autres: une meilleure analyse des situations à risque, la capacité de prise de décision face à celles-ci, la possibilité de demander de l'aide ou de se défendre, ou encore celle de signaler ou de porter plainte (Mechling, 2008; Khemka, Hickson \& Reynolds, 2005).

Enfin, même si parler de maltraitance reste difficile et menaçant pour les protagonistes et l'institution en cause, nous jugeons préférable de le faire plutôt que d'évoquer uniquement la bientraitance. En effet, plusieurs institutions privilégient cette voie d'entrée pour la formation de leur personnel; considérée comme positive et valorisante, elle permettrait de dresser un catalogue de bonnes pratiques. Se centrer sur les bonnes pratiques est certainement fondé, mais il importe de traiter aussi de la maltraitance. Une formation axée uniquement sur la bientraitance comporterait le risque de contribuer à la négation de l'existence d'actes maltraitants commis par les professionnel.le.s. Pour identifier ceux-ci, il est essentiel d'en admettre la présence et de pouvoir en parler sans craindre d'être mis à l'écart du système. A cet égard, la formation représente une voie de choix. 


\section{Conclusion}

Ces deux recherches, réalisées dans le cadre institutionnel et dans celui de la formation tertiaire, permettent d'entrevoir la nécessité d'actions et de formation. Cela afin de prévenir un phénomène qui risque, avec la promotion de l'inclusion sociale telle qu'elle est encouragée dans la dernière Convention internationale de l'ONU relative aux droits des personnes handicapées (2006), de se reproduire plus largement dans le contexte élargi de la cité. La ratification en 20 I4 par la Suisse de cette convention suggère la nécessité d'une réflexion approfondie afin de prévenir la maltraitance dans différents contextes de vie où se retrouvent les personnes vulnérables.

Afin d'empêcher la survenue de la maltraitance en milieu institutionnel, son existence doit être reconnue, sans mettre en péril les personnes et l'organisation concernées. La maltraitance constitue un phénomène complexe, qui implique la responsabilité de nombreux acteurs et actrices et, la plupart du temps, l'organisation et le fonctionnement de l'institution. De plus, sa méconnaissance pourra être accentuée selon les normes et la culture de la société ou elle s'exerce.

Pour être contrée, elle nécessite d'être considérée dans sa complexité par une analyse des différents sous-systèmes concernés visant à la réduction des facteurs précipitants et favorisant la mise en place de facteurs protecteurs. Dans les milieux d'accueil socio-éducatifs ou médico-sociaux, elle est régulièrement commise sans mauvaise intention, voire avec la sensation de «bien traiter». Elle est parfois invisible, car coulée dans une routine de fonctionnement qui n'empêche pas de répondre aux besoins physiologiques des personnes. Les repas, l'hygiène, les soins peuvent y être assurés de façon satisfaisante tout en déniant l'autre, son être, ses sentiments, ses désirs, ce qui l'anime et le fait vivre, en ne lui offrant aucune reconnaissance sociale. L'accompagnement des personnes vulnérables devrait dépasser l'unique but de "maintenir en vie» pour contribuer à ce que la personne vive sa vie en ayant le sentiment d'être reconnue et considérée (Masse, 20I4).

Les retombées des programmes de formation demeurent insuffisamment connues. A ce jour, il reste à développer des formations sur le thème de la prévention de la maltraitance et à les analyser afin de permettre de mieux comprendre comment elle advient. Il reste à vérifier quelles sont les mesures les plus efficaces pour rendre l'accompagnante et l'accompagnant 
vigilant.e à ses actes, à ceux de ses pairs et au fonctionnement institutionnel afin que, lorsque le basculement s'est produit, la maltraitance puisse être stoppée avec un minimum de conséquences pour la victime et son entourage. Pour prévenir la maltraitance en milieu institutionnel et en communauté, toutes les personnes qui y travaillent ou y vivent ainsi que leurs proches devraient être formées. Certains programmes pourraient être mixtes ou viser des populations distinctes, ou encore les réunir en alternance. L'expérimentation de différents programmes en formation initiale ou en formation continue permettront de préciser les contenus à traiter, les populations cibles ainsi que les pédagogies les plus efficaces à envisager.

Aujourd'hui, les milieux institutionnels reconnaissent progressivement l'existence de la maltraitance, la définissent dans des procédures de leur système de gestion, créent des chartes. Certaines institutions appliquent des sanctions lorsqu'elle survient. Quelques-unes ont créé des dispositifs internes pour la signaler, même si ces derniers présentent bien souvent des lacunes d'accessibilité (Jordan-Ionescu, I999; Masse et Petitpierre, 20I I). Si prévenir la maltraitance avant même sa survenue (niveau primaire) et la stopper dès son apparition (niveau secondaire) demeure un objectif à atteindre, il importe aujourd'hui de se pencher sur la manière de la traiter et d'aider les actrices et acteurs et les victimes lorsque la maltraitance est survenue (niveau tertiaire). Comment aider les personnes qui la subissent sans oublier les pairs résidant dans le même lieu, les autres professionnel.le.s qui y travaillent, les témoins directs et indirects? Quel soutien offrir aux auteures et auteurs de ces actes? Ce sujet reste peu traité dans les recherches et les travaux sur les personnes dépendantes et vulnérables.

Au-delà de ce qui doit être proposé comme formation pour prévenir la maltraitance dans les milieux institutionnels, ce travail questionne l'accueil des personnes vulnérables en institution. Ces milieux sont-ils propices au déploiement d'actes de maltraitance? Il demeure évident que l'ouverture des institutions et l'appartenance à un environnement social élargi représentent une protection contre la survenue des maltraitances. Les personnes dépendantes et vulnérables devraient pouvoir bénéficier de différentes possibilités d'hébergement afin d'obtenir une meilleure réponse à leurs besoins. Les petites structures et l'accueil en milieu familial ne garantissent toutefois pas une protection face aux abus. Nos sociétés peinent à trouver des modalités d'accueil adaptées aux besoins de chacune et chacun. Par-delà les mots, comment devenir une société inclusive, accordant 
à chacun·e la place qui lui revient au sein d'un groupe d'appartenance demeurant bienveillant malgré ses différences et sa vulnérabilité?

Si l'agressivité fait partie de la vie et, dans certains cas, contribue à l'affirmation de soi ou à la défense de ses biens ou territoires, lorsqu'elle s'exprime par la négation et la déshumanisation de l'autre, en devenant néfaste pour son développement ou son bien-être, elle devient de la maltraitance.

Dans un monde meilleur, il serait possible de vivre dans une société de la reconnaissance (Honneth, 20Io), laquelle s'appliquerait à chaque individu, y compris aux personnes les plus vulnérables. Comme le rappelle Charles Gardou: «Il n'y a ni vie minuscule, ni vie majuscule» (2012: 84). En conséquence, chaque existence devrait être appréhendée dans l'optique de sa contribution à l'humanité, et il s'agit là d'une responsabilité qui incombe à chacune et chacun d'entre nous.

\section{Références bibliographiques}

Abric, J.-C. (I994). Les représentations sociales: aspects théoriques. Dans

J.-C. Abric (Ed.), Pratiques sociales et représentations (pp. I I-35). Paris:

Presses Universitaires de France.

Bellis, M. A., Hughes, K., Jones, L. et al. (2012). Prevalence and risk of violence against adults with disabilities: a systematic review and meta-analysis of observational studies. The Lancet, 379(9826), I62I-I629. Consulté le 29.I0.20I3 sur http://download.thelancet.com/pdfs/journals/lancet/PIISoI406736I I6I85I5.pdf

Belsky, J. (I993). Etiology of child maltreatment: a developmental-ecological analysis. Psychological Bulletin, I I4(3), 4I3-434.

Bronfenbrenner, U. (I979). The ecology of human development. Experiments by nature and design. Cambridge: Harvard University Press.

Bronfenbrenner, U. (I996). Le modèle «Processus-Personne-Contexte-Temps» dans la recherche en psychologie du développement: principes, applications et implications. Dans R. Tessier \& G. M. Tarabulsy (Dir.), Le modèle écologique dans l'étude du développement de l'enfant (pp.9-59). Sainte-Foy: Presses de l'Université du Québec.

Brown, H. (2003). Protection des adultes et des enfants handicapés contre les abus. Strasbourg: Editions du Conseil de l'Europe.

Conseil de l'Europe (1992). La violence envers les personnes âgées. Strasbourg: Conseil de l'Europe.

Corbet, E. (2000). Les concepts de violence et de maltraitance. Actualité et dossier en santé publique, 3 I, 20-25.

Faerber, R. (2002). Le groupe d'apprentissage en formation à distance: ses caractéristiques dans un environnement virtuel. Dans F. Larose \& T. Karsenti (Ed.), La place des TIC en formation initiale et continue à l'enseignement: bilan et perspectives (pp.99-I28). Sherbrooke: Université de Sherbrooke, CRP. 
Hughes, K., Bellis, M. A., Jones, L., Wood, L., Bates, G., Eckley, L. \& Officer, A. (20I 2). Prevalence and risk of violence against adults with disabilities: a systematic review and meta-analysis of observational studies. The Lancet, 379(9826), I62II629. Consulté le 29.I0.20I3 sur http://download.thelancet.com/pdfs/journals/ lancet/PIISoI406736I I6I85I5.pdf

Jodelet, D. (1997). Représentation sociale: phénomènes, concept et théorie. Dans S. Moscovici (Dir.), Psychologie sociale. Paris: Presses Universitaires de France.

Gardou, C. (20I2). La société inclusive, parlons-en! Il n’y a pas de vie minuscule. Toulouse: Erès.

Honneth, A. (2010). La lutte pour la reconnaissance. Paris: Cerf.

Houssaye, J. (2000). Le triangle pédagogique. Théorie et pratiques de l'éducation scolaire. Berne: Peter Lang.

Jordan-Ionescu, C. (I999). Ethique et signalement de la maltraitance. Bulletin de psychologie, 52 (5), 556-56I.

Khemka, I., Hickson, L. \& Reynolds, G. (2005). Evaluation of a decision-making curriculum designed to empower women with mental retardation to resist abuse. American Journal on Mental Retardation, I IO(3), 193-204.

Krug, E. G., Dahlberg, L. L., Mercy, J. A., Zwi, A. \& Lozano-Ascencio, R. (2002). Rapport mondial sur la violence et la santé. Genève: Organisation mondiale de la santé. Consulté le or.09.20I4 sur http://www.who.int/violence_injury_prevention/violence/world_report/en/full_fr.pdf

Masse, M. (20I4). Quel programme de formation pour prévenir la maltraitance en milieu institutionnel?: analyse des retombées d'une formation sur les représentations de la maltraitance, ses facteurs de protection et les actions développées. [Thèse de doctorat]. Lyon: Université Lumière Lyon 2.

Masse, M. \& Petitpierre, G. (20 I I). La maltraitance en institution. Les représentations comme moyen de prévention. Genève: Editions ies.

Masse, M., Petitpierre, G., Vidon, C. \& Jossevel, J.-D. (2009). Prévention de la maltraitance en institution. Collaboration autour de l'élaboration d'un support pédagogique. Dans V. Guerdan et al. (Ed.), La participation, pour une inclusion des personnes en situation de handicap (pp.642-659). Berne: Peter Lang.

Mechling, L. (2008). Thirty year review of safety skill instruction for persons with intellectual disabilities. Education and training in developmental disabilities, 43(3), 3II-323.

Moscovici, S. (1984). The phenomenon of social representations. Dans R. M. Farr \& S. Moscovici (Ed.), Social representations (pp.3-69). Cambridge: Cambridge University Press.

Orellana, I. (2006). Les perspectives d'éducation relative à l'environnement en contexte de communauté d'apprentissage. Dans J.-C. Kalubi (Dir.), L'éducation en débats: analyse comparée. Communautés apprenantes et interventions éducatives (vol.4). Bienne: HEP Béjune.

Organisation des Nations unies. (2006). Convention relative aux droits des personneshandicapées. Consulté le or.09.20I4 sur http://www.admin.ch/opc/fr/classifiedcompilation/201 22488/index.html 
Organisation des Nations unies. (1989). Convention internationale des droits de l'enfant. Consulté le 25.09.2006 sur http://www2.ohchr.org/french/law/crc.htm

Organisation mondiale de la santé. (I986). Charte d'Ottawa pour la promotion de la santé. Consulté le I5.05.2006 sur http://www.euro.who.int/_data/assets/pdf_ file/o003/r 29675/Ottawa_Charter_F.pdf

Organisation mondiale de la santé. (2002). La prévention du suicide: indications pour les professionnels des médias. Consulté le 25.09.2006 sur http://whqlibdoc.who.int/ publications/2002/9242545619_fre.pdf

Papart, J.-P. (2006). Lien social et santé mentale. Chêne-Bourg: Médecine \& Hygiène.

Petitpierre, G. (2009). Maltraitance et déficience mentale: des démarches de prévention passives à la prévention participative. Dans V. Guerdan, G. Petitpierre, J.-P. Moulin \& M.-C. Haelewyck (Ed.), Participation et responsabilités sociales: un nouveau paradigme pour l'inclusion des personnes avec une déficience intellectuelle (pp.660-669). Berne: Peter Lang.

Petitpierre, G., Masse, M., Martini-Willemin, B.-M. \& Delessert, Y. (2013). A complementarity of social and legal perspectives on what is abusive practice and what constitutes abuse? Journal of policy and practice in intellectual disabilities, Io(3), 196-206.

Tomkiewicz, S. (2002). Violences dans les institutions pour enfants, à l'école et à l'hôpital. Dans M. Manciaux, M. Gabel, D. Girodet, C. Mignot \& M. Rouyer (Ed.), Enfances en danger (pp.309-360). Paris: Fleurus.

Trefois, P. (2003). L'usage de la peur: une approche légitime en promotion de la santé? Bruxelles Santé, $n^{\circ}$ spécial, I9-30. Consulté le i0.07.2006 sur http://www. questionsante.org/o3publications/charger/peuretprevention.pdf 

Et au-delà

Troisième partie 



\section{A la recherche du livre perdu}

Ecrire et publier un ouvrage issu

d'une recherche en sciences sociales

\section{Stéphanie Fretz}

\section{A Monique}

«Ils viennent aux livres poussés par un désir.

Quand ils les quittent, parfois quelque chose a changé en eux.»

Alexandre Mirénowicz,

bibliothécaire à la Fondation Jan Michalski (2016)

\section{Introduction ${ }^{1}$}

Publier des livres de sciences humaines et sociales (SHS) peut apparaître comme dépassé: ce type d'ouvrages est réputé peu rentable, souvent trop long, difficile à diffuser; imprimer un ouvrage scientifique est considéré par certaines instances scientifiques comme secondaire. Pourquoi diable, donc, continuer de publier des livres papier qui coûtent cher et qui se vendent peu? Pourquoi donc s'acharner de la sorte? Sans doute, faut-il attribuer cet entêtement à des «nostalgiques " qui ne savent pas s'adapter à la marche du monde et qui ont peur du changement.

Et pourtant, elles et ils sont encore nombreuses et nombreux - maisons d'édition, auteur·e·s, lectrices et lecteurs - à croire qu'une recherche, si elle a besoin de temps pour se déployer (comme le développe Claudio Bolzman

1 Que soient ici remerciés Monica Battaglini, Marc Breviglieri, Stéphane Michaud, responsables de collection, et Marion Schmitz, chargée de la promotion et de la commercialisation des ouvrages des Editions ies, sans lesquel.le.s ce projet éditorial ne pourrait être mené à bien. Que soient aussi remercié.e.s les auteur.e.s qui ont l'audace de faire confiance aux Editions ies. 
dans sa contribution, pp.27-43), a aussi besoin de pages pour être présentée, expliquée, analysée, transmise. Tout ne peut tenir en I80 secondes ou I40 caractères. La pensée ne se stocke pas sous formes de données. Certain.e.s chercheur.e.s considèrent qu'une partie de l'analyse qu'elles et ils font de leur sujet s'effectue à partir d'un processus d'écriture et de réécriture qui exige de reprendre les termes de la problématique, de peser chaque mot, de chercher l'exemple, de donner des détails, de construire puis de déconstruire l'organisation du texte et de faire la synthèse des résultats, tout à la fois. D'aucun·e.s vont même jusqu'à penser que 40000 signes, espaces compris (le format de la plupart des articles), sont encore trop peu pour développer l'ensemble des hypothèses, résultats et pistes abordés dans une recherche. Ils et elles sont encore nombreux à croire au temps long et lent de l'écriture, de la réécriture, puis de la lecture, voire de la relecture. A croire que tourner des pages et annoter des textes n'est pas anodin. Que la valeur (voire l'efficience) d'un ouvrage ne se mesure pas à au nombre de citations qu'on comptabilise dans les moteurs de recherche, mais à la maturation ou à la critique de textes qui permettent de construire son identité de lectrice ou lecteur, de chercheur.e, d'enseignant·e, de citoyen.ne.

\section{Une maison d'édition dans une haute école}

Les Editions ies de la Haute école de travail social de Genève existent depuis plus de quarante ans (le premier ouvrage est paru en I97I). Ces éditions sont étroitement liées à la recherche puisque leur création est concomitante à celle du Centre de recherche sociale (crée en I968). L'idée première associée à la création de ces éditions était de mettre en avant les travaux de recherche, et notamment les travaux de recherche d'étudiant.e.s.

Actuellement, dans le cadre de la politique éditoriale des Editions ies, la valorisation de la recherche est toujours au cœur de sa mission; la valorisation de pratiques pédagogiques et professionnelles (par des professionne.le.s issu.e.s du terrain ou de l'enseignement) est aussi encouragée, ainsi que la publication d'ouvrages classiques de sciences humaines et sociales (SHS) ou d'essais plus personnels dans les domaines ayant trait au travail social et à la petite enfance. ${ }^{2}$

Au fil des ans, les collections des Editions ies ont évolué, de même que les contenus. Si le lien entre théorie et pratique a toujours été au centre des ouvrages, les modalités de publication ont changé. On compte actuellement 
trois collections actives, et une en gestation, mais sept ont existé et se sont éteintes depuis la création du service. ${ }^{3}$ La dernière collection à disparaître, ou plutôt à se transformer, est celle de la «Collection du Centre de recherche sociale», dans laquelle étaient publiés, le plus rapidement possible, des rapports de recherche relativement bruts. Le fait que ces rapports ne soient plus exigés par les mandant.e.s ou les instances finançant la recherche et le fait que de tels rapports puissent être mis en ligne sur des plateformes dédiées font que cette collection s'est arrêtée et que les ouvrages publiés à partir de recherches ont pris une autre forme dans la collection «Le social dans la cité».

Les publications sont un des vecteurs de diffusion de la recherche. A travers l'évolution des modes éditoriaux, on perçoit l'évolution du monde scientifique (et vice versa). La politique éditoriale (comme l'enjeu de cet ouvrage) reflète l'oscillation - ou l'équilibre - entre l'accent mis sur l'insertion des publications dans la cité (tournée plutôt vers un public hors formation, de politiques, de travailleuses et travailleurs sociaux, etc.), une volonté d'alimenter l'enseignement en priorité (et de s'adresser aux enseignant.e.s ou étudiant.e.s) ou encore de stimuler la recherche elle-même (en s'adressant à d'autres scientifiques des SHS). Pour une institution comme la Haute école de travail social de Genève, soutenir l'existence d'un service de publication en son sein relève d'une politique institutionnelle affirmée et permet de mettre en avant la diversité des manières de mener des recherches, de les valoriser et de réinjecter les retombées de celles-ci notamment vers l'enseignement et/ou la cité.

Dans les lignes à suivre, nous nous attacherons à la manière dont un ouvrage - plus spécifiquement un ouvrage lié à une recherche - est actuellement publié aux Editions ies. Ce processus sera appréhendé selon trois axes: tout d'abord, à partir de la motivation à publier (Le moteur du livre), puis en fonction des destinataires de l'ouvrage (A qui profite le livre?) et, enfin, selon les modes de diffusion pour atteindre ceux-ci (Accessibilité potentiellement illimitée versus diffusion ancrée).

2 Pour plus de détails, voir les pages de présentation des Editions ies. Consulté le 31.0I.20I7 sur https://www.hesge.ch/hets/editions-ies/presentation-et-historique

3 Le nom des différentes collections est disponible sur le site des Editions ies. Consulté le 3I.OI.20I7 sur https://www.hesge.ch/hets/editions-ies/informationsgenerales/collections 


\section{Le moteur du livre}

Le désir de liure

Au sein de l'équipe éditoriale des Editions ies, il est souvent question du "désir de livre». Le désir que le ou la chercheur.e peut éprouver à voir son travail de recherche se concrétiser sous la forme d'un ouvrage, de se positionner comme auteur.e (et non pas seulement comme «contributrice» ou "contributeur»). Le désir de partager sa pensée et son travail avec de potentiels lecteurs et lectrices, dans une forme particulière. Le désir d'être lu.e par ses pairs - mais aussi par d'autres. Le désir de donner une visibilité au fruit de son labeur. Le désir de contribuer au débat autour d'une question donnée. Ce désir dépasse l'exigence d'établir un rapport, de rendre des comptes ou de justifier de l'aboutissement d'une recherche pour que celle-ci soit comptabilisée ou administrativement bouclée.

Le désir de publier contient le désir d'une rencontre. La rencontre entre un.e chercheur.e et son sujet, entre un.e chercheur.e et d'autres chercheur.e.s, entre un.e chercheur.e et ses lectrices et lecteurs. La potentielle rencontre est donc inscrite dans l'acte rédactionnel.

Publier, c'est aussi poser des éléments, laisser des traces, rendre publics des résultats, exposer des analyses de phénomènes sociaux et les inscrire dans la durée (acte relevant d'une certaine résistance, à l'ère de l'obsolescence programmée). Lorsque ces éléments sont posés, on peut s'y référer, les prolonger, les discuter, alimenter la réflexion, construire une pensée collective. Publier, c'est aussi s'exposer à la critique et la forme du livre permet d'affiner ses développements et arguments davantage que le format d'un article. Rendre sa pensée publique, c'est encore la fixer dans un temps et dans un environnement donnés.

Publier un ouvrage, c'est aussi une aventure de longue haleine. Qui a déjà publié sait la tâche ardue, parfois ingrate et fastidieuse. Il faut reprendre son texte maintes fois. Le texte est expertisé sous l'angle du domaine dans lequel il se situe, de la manière de traiter la problématique, de sa pertinence par rapport à la recherche sur des sujets similaires, selon son aspect formel, sur sa lisibilité, sur la clarté de l'exposition... Autant d'aspects qui génèrent différentes étapes de réécriture.

Il faut donc une motivation et un temps certains pour se lancer dans cette entreprise. 


\section{Un processus d'accompagnement}

Les auteur.e.s potentiels qui se présentent aux Editions ies peuvent se trouver à différentes phases de leur «désir de livre». Certain.e.s chercheur.e.s se présentent avec un texte rédigé, d'autres avec un projet en cours et prennent contact au cours de l'élaboration de la recherche elle-même.

Le processus de publication aux Editions ies mêle à la fois, concertation, expertise collective et accompagnement personnalisé.

Lorsqu'un manuscrit ou projet est soumis, il est d'abord appréhendé par le comité éditorial puis, lorsque le manuscrit est considéré comme suffisamment avancé, il est soumis à une commission de lecture. ${ }^{4}$

Le comité éditorial va tout d'abord s'assurer de la pertinence du texte avec la politique éditoriale de la maison et tenter de voir dans quelle collection le manuscrit pourrait s'insérer. Ce comité est composé de la responsable du service, de la chargée de diffusion et de la promotion et des responsables de chaque collection, professeur.e ou maître d'enseignement. Suivant le type de texte, trancher entre une collection et une autre n'est pas toujours évident et un texte peut être pressenti dans une collection puis être publié dans une autre, suivant l'orientation finalement prise par son auteur.e.

Lors des réunions du comité éditorial, un premier regard collectif est donc posé sur le manuscrit. La qualité d'écriture du texte est, bien sûr, prise en considération, mais sont aussi et surtout considérés le lien de la problématique traitée avec le travail social, la psychomotricité ou des pratiques pédagogiques, l'apport du texte au champ concerné, ainsi que l'intérêt pour au moins un public cible des Editions - à savoir les professionnel.le.s, chercheur.e.s et enseignant.e.s, étudiant.e.s, actrices et acteurs de la politique sociale. A ce stade, les Editions ies décident d'entrer en matière, ou pas, dans l'accompagnement d'un projet vers la publication.

Un.e des responsables de collection se met ensuite en contact avec l'auteur.e pour reprendre certains points du manuscrit. Un travail de réécriture, déconstruction, reconstruction (travail détaillé ci-après) est entrepris et peut durer plusieurs mois, jusqu'à ce que, de concert, l'auteur.e et 
le ou la responsable de collection considèrent que le texte peut être présenté à la commission de lecture.

Cette commission est composée du comité éditorial et de membres externes et internes à la Hets. Ces membres ont été choisis pour leur profils professionnels variés; certain.e.s sont issus du milieu du livre - bibliothécaire ou libraire -, d'autres du milieu académique, ou encore du milieu professionnel du travail social. Des expert.e.s externes du thème, des pairs issus du milieu académique sont aussi sollicités ponctuellement. A travers cette diversité de regards sur les manuscrits, à travers cette manière de considérer l'objet sous différents angles, les publications reflètent les préoccupations des HES suivantes: quelle articulation entre des concepts théoriques et les pratiques professionnelles le manuscrit offre-t-il? Comment l'ouvrage pourra-t-il être utile à la cité, aux politiques, aux professionnel.le.s, aux usagères et usagers? Comment le texte alimente-t-il le champ académique? Et celui de la formation?

Après discussion, cette commission émet des recommandations, mais la décision de publication revient au comité éditorial, et plus particulièrement à la personne responsable de collection. Le manuscrit peut être accepté quasiment tel quel, avec des modifications formelles ou de contenu (plus ou moins importantes), ou peut aussi être refusé. Ces derniers cas sont désormais plus rares, étant donné que le comité éditorial fait office de premier filtre. En revanche, un.e auteur.e peut renoncer aux modifications demandées si elles lui semblent non pertinentes ou trop coûteuses (en temps surtout) et retirer son projet.

Toutes ces étapes visent une plus-value en termes de contenu: une problématisation clairement posée et traitée, un recentrage si nécessaire, un ciblage du public, une clarté et une précision du propos, une lisibilité maximale. Une fois le manuscrit accepté, le texte est aussi soigné formellement; il est relu pour des questions de cohérence, de syntaxe, puis vient la chasse aux coquilles, à la ponctuation erronée, aux erreurs typographiques, à l'orthographe créative, etc. La bibliographie est vérifiée et unifiée. Un soin particulier est apporté à la mise en page et à l'impression. Et désormais, il faut ajouter à ces éléments le formatage pour l'édition électronique (qu'elle soit en libre accès ou non) et le référencement. Plus largement, la diffusion et la promotion sont des aspects fondamentaux du travail de l'éditeur.e. Ces éléments formels et promotionnels font aussi partie de cette plus-value éditoriale. L'objet livre est choyé afin que la lecture soit la plus agréable possible. 
Il faut aussi savoir valoriser le manuscrit auprès d'organismes subventionneurs, qu'il s'agisse du Fonds national suisse de la recherche scientifique (FNS) ou de la HES-SO en ce qui concerne les publications issues de recherches menées au sein de la Hets, ou les universités ou institutions auxquelles appartiennent les auteur·e•s, voire des fondations privées, les terrains ou les instances politiques. Là aussi, il faut un certain savoir-faire (et du temps) pour établir les budgets, les plans de financement et les dossiers qui inspireront confiance aux organismes financeurs.

Difficile transformation du rapport de recherche en liure

Travail de réécriture

Classiquement, un rapport de recherche se présente comme suit: une introduction pose la problématique et annonce le plan, on rend compte de la littérature convoquée, de la méthodologie déployée, des résultats obtenus, on procède à l'analyse de ceux-ci et on insère une bibliographie. Le rapport de recherche apporte une justification scientifique aux résultats, il décrit principalement le protocole de recherche suivi. Or, le développement du pôle numérique est devenu le mode de prédilection de diffusion de ce type de rapport. L'accessibilité à des blogs de recherche (du type Carnets de recherche du portail OpenEdition), ${ }^{5}$ à des archives ouvertes, aux sites des universités, à la littérature grise, à des revues en ligne sont autant de canaux qui dispensent des rendus de recherche. Les «rapports» de recherche ne sont pas forcément l'objet de la publication d'un livre, «on les publie en ligne». Néanmoins, les Editions ies, parmi d'autres, continuent de croire à l'importance de la publication de monographies issues de travaux de recherche. Problématique, épistémologie et méthodologie constituent alors un matériau qui peut se trouver dispersé au fil du texte plutôt qu'organisé en parties distinctes; cet appareil est réduit au profit de l'analyse des résultats, des éléments qui peuvent en être retirés, des clés de lecture possibles sur une réalité donnée, de pistes d'action à envisager.

Les propositions de manuscrits sont rarement acceptées tels quels. Les rapports de recherche sont souvent longs et détaillés. Si le détail du processus est essentiel à la recherche, il l'est en général moins à la publication.

5 Consulté le 31.0I.20I7 sur https://www.openedition.org/catalogue-notebooksrecherche/open-access/Pages/default.aspx 
Les auteur·e·s sont souvent amené·e·s à réduire leur texte, à en supprimer certaines parties ou à les synthétiser. L'utilisation des citations d'entretiens doit parfois être affinée, l'analyse des propos cités sera parfois développée, des implicites explicités.

\section{Une temporalité contrariée}

Lorsqu'un·e auteur.e soumet un rapport de recherche aux Editions ies, la recherche est, de fait, déjà terminée (et ce, parfois depuis longtemps). Les exigences de la maison d'édition ne sont pas celles de l'organisme mandant et l'expertise des textes peut prendre un certain temps. Au moment où un.e chercheur.e doit reprendre son texte, comme auteur.e, elle ou il est en général déjà plongé.e dans une nouvelle recherche, pris·e par d'autres mandats. Il faut alors une forte dose de ténacité et de persévérance pour maintenir vivace le «désir de livre». On peut se demander ce qu'il en sera de ce désir au moment de la diffusion dudit livre, quelques mois plus tard, l'auteur.e devant s'impliquer dans la promotion de son ouvrage en sollicitant son réseau, en faisant des présentations publiques, en osant recommander l'ouvrage dans des cours, etc.

Le monde académique l'exige, à un instant $\mathrm{T}$, il faut valoriser la recherche $\mathrm{A}$, mener les recherches BI voire B2 et B3, tout en soumettant et en cherchant des financements pour la recherche $C$ !

Penser le liure en amont dans la recherche

Aux Editions ies, nous sommes confronté·e·s aux difficultés de réécriture, de frustration, de découragement, de manque de temps, de décalage entre l'actualité de la publication et celle de la recherche. C'est pourquoi nous encourageons les futurs auteur.e.s à envisager la forme de publication souhaitée alors qu'ils réalisent leur recherche, nous les encourageons à prendre contact avec l'équipe éditoriale avant que celle-ci soit achevée. De cette manière, il est possible d'orienter, non pas la recherche, mais le rendu de celle-ci. Ensemble, responsable éditorial.e et auteur.e peuvent envisager un plan, un aspect à développer, à mettre en lumière. En cherchant à définir ensemble le lectorat envisagé, l'écriture elle-même sera influencée.

Ce travail d'accompagnement de projet est conséquent pour l'équipe éditoriale, mais également passionnant. Il permet un gain de temps substantiel dans la publication et un gain de temps pour les auteur.e.s, 
dont le travail de réécriture sera moindre. La maison d'édition agit alors comme une "accoucheuse» de livre et cherche à établir un dialogue et une confiance avec l'auteur.e.

Le plaisir de lire

Le plaisir de lecture n'est pas une question purement hédoniste, que d'aucun·e.s pourraient trouver déplacée lorsqu'il est question de littérature scientifique. Outre le fait qu'un lien entre plaisir et apprentissage existe, il est ici question de lisibilité du texte et de plaisir intellectuel. En effet, pour entrer dans la pensée d'autrui, il faut que l'invite du lecteur ou de la lectrice soit réelle, que la problématique soit claire, le déroulement de la pensée limpide et balisé, la démonstration argumentée, etc. Lorsque ces conditions sont réunies, le lecteur ou la lectrice peut se confronter aux hypothèses, méthodologies, théories, problématiques et résultats énoncés, se positionner et entamer un dialogue avec le texte. Là encore, l'éditrice ou l'éditeur peut accompagner l'auteur.e pour parfaire son texte en vue d'une meilleure intelligibilité, en posant plus clairement la problématique, en replaçant celle-ci dans un contexte plus large ou en réorganisant la structure du texte, par exemple.

\section{A qui profite le livre}

\section{Un laboratoire de la pensée sociale}

En tant que service de publication d'une haute école, les Editions ies ont à l'esprit les missions qui sont celles de son établissement: participer à la réflexion dans les domaines en lien avec le travail social, alimenter les enseignements, s'insérer dans la cité et apporter aux actrices et acteurs de la politique sociales des éléments permettant des décisions éclairées, valoriser le travail social comme discipline académique.

La problématique de cet ouvrage - le lien entre la recherche et l'enseignement d'une part et la cité d'autre part - est également au cœur des préoccupations d'un service de publication tel que celui des Editions ies. Les Editions ies fonctionnent comme une sorte de laboratoire de la pensée sociale ou comme courroie de transmission. La publication de recherches, notamment, permet de renforcer le lien entre milieu académique, enseignement et cité. 
En considérant à qui est destiné l'ouvrage, les auteur.e.s mettent l'accent sur un aspect ou l'autre selon le texte qu'ils désirent publier.

Les destinataires

Un ouvrage, quel qu'il soit, ne peut se concevoir sans imaginer son lectorat. Pour qui écrit-on? Pour l'organisme mandant, pour les collègues chercheur.e.s, pour les formateur.e.s, pour les étudiant·e.s, pour les contradicteur.e.s, pour les professionnel.le.s? Quel est le niveau de compétence des destinataires visés? Quelle est sa connaissance du sujet? Quel message veut-on faire passer?

La question de la ou du destinataire est sans cesse au cœur des expertises réalisées au sein des Editions ies. C'est en ayant cet aspect-là à l'esprit que l'ouvrage pourra se façonner, tirer son fil rouge et élaborer sa forme. Lorsque le ou la chercheur.e a le ou la destinataire à l'esprit, il sait que certains aspects plutôt que d'autres sont à expliciter. Si le public visé est large, il devra éviter un jargon de spécialiste et clarifier certains implicites.

L'ouverture vers un lectorat large n'est pas toujours aisée à mener car, si l'ouvrage vise un tel public, il vise aussi souvent les pairs de l'auteur.e, pour qui l'explicitation de certaines notions peut apparaître superflue et répétitive. La publication jongle parfois avec une diversité de publics dont les niveaux de connaissances peuvent être hétérogènes. Cela constitue une gageure pour ce type de livre. Des encarts, des synthèses, des parties introductives ou des résumés, des notes, un glossaire peuvent permettre à des lectrices et lecteurs ayant des niveaux de connaissances différents de s'approprier le contenu offert.

\section{L'importance d'une politique éditoriale}

L'insertion dans un programme éditorial ou dans une collection définie permet aussi de clarifier l'horizon d'attente du lectorat. Aux Editions ies, la collection consacrée en priorité à la recherche est la collection «Le social dans la cité». Le nom même de la collection évoque bien l'ambition de diffuser les fruits de la recherche au-delà du milieu académique et implique une écriture accessible.

Un ouvrage sur l'autorité dans les institutions sociales (Parazelli \& Ruelland, 20I7) est paru dernièrement dans la collection «Le social dans la cité». Cet ouvrage est issu d'une recherche qualitative menée essentielle- 
ment au Québec, dans un contexte institutionnel particulier. Les auteur·e.s rendent compte de résultats, mais sollicitent également la littérature autour de cette notion, en offrent une approche socio-historique et font appel à l'approche d'intervention sociopsychanalyste de Mendel. Si cet ouvrage se base sur une recherche, son propos dépasse celui du rapport et a pour objectif de réintroduire de la démocratie dans les milieux du travail social. L'analyse des résultats peut, dès lors, avoir une portée au-delà du contexte étudié.

La publication de résultats de recherche ne se cantonne néanmoins pas à une seule collection; selon l'axe ou la forme donnée à une publication, des textes peuvent aussi paraître dans d'autres collections. Roms en cité (Battaglini et al., 20I5), par exemple, issu d'une recherche-action, met en avant la méthodologie de recherche adoptée. Les chercheures-actrices ont sollicité des techniques de participation par groupes de parole (thérapie communautaire systémique et intégrative, approche centrée sur la solution) pour entrer en relation avec des personnes roms. La mise en avant du travail effectué sur le terrain et le fait que cette démarche puisse être reproduite et adoptée par des actrices et acteurs de terrain travaillant avec des personnes en situation de vulnérabilité ont fait que la collection "Pratique.s", axée davantage sur les pratiques pédagogiques et professionnelles, a été retenue pour publier cette recherche. De même, Articuler genre et diversité (Eckmann \& Földhazi, 2013), après un tour d'horizon de plusieurs pays mettant en œuvre des pratiques pédagogiques visant l'intégration dans la diversité de toutes et tous, centre son propos sur des questions de mise en œuvre et établit des fiches à utiliser de manière concrète dans la préparation des enseignements. Cet aspect pragmatique du texte a également orienté l'ouvrage vers la collection «Pratique.S». Dans les deux cas, c'est le caractère duplicable et applicable dégagé des recherches qui a déterminé ce choix de collection.

La traduction de l'enquête menée dans les années I960 par Aaron Cicourel, The Social Organization of Juvenile Justice (20I8) - qui a fait date pour les études concernant la délinquance et la déviance juvénile - paraît dans la collection «Le geste social », qui accueille des ouvrages prenant la forme d'essais. Ici, c'est en considérant le caractère «classique» de cet ouvrage que cette collection a été retenue. La publication de cette traduction (cinquante ans après la première édition) souligne la pérénnité qu'un ouvrage peut acquérir avec le temps.

La publication de résultats de recherche peut prendre plusieurs formes, il peut s'agir d'un article ou d'un livre. Un ouvrage, quant à lui, peut prendre plusieurs formes et donner lieu à divers types de publications: ouvrage 
spécialisé ou ouvrage de vulgarisation, mettant en avant une méthodologie ou s'attachant davantage à l'analyse des résultats, à proprement parler, etc. Il peut être destiné en priorité à un type de lectorat ou à un autre, à un type de lecture ou à une autre (lecture exhaustive ou par bribes). Nous essayons de maintenir et de marquer cette diversité au sein des Editions ies. Chaque collection a une identité clairement définie et, suivant le visage que l'auteur.e souhaite donner à son ouvrage, suivant la ou le destinataire visé.e en priorité, une collection sera favorisée plutôt qu'une autre. Cette définition claire des collections oriente la lecture et permet une catégorisation des textes qui en facilite la réception; la connaissance des collections permet de clarifier l'attente quant au type de texte publié, ou de s'orienter vers une collection ou une autre en fonction desdites attentes. La publication d'ouvrages au sein d'un catalogue défini selon une ligne éditoriale précise et structurée en collections spécifiques manifeste la recherche de cohérence et d'intelligibilité de la maison d'édition.

\section{Accessibilité potentiellement illimitée versus diffusion ancrée}

La publication en ligne en libre accès offre une accessibilité potentiellement illimitée et internationale. Néanmoins, ce mode de diffusion - quand il atteint sa cible - concerne essentiellement les actrices et acteurs de la communauté scientifique, se fait principalement en anglais et atteint un public relativement homogène. Paradoxalement, une diffusion plus traditionnelle de livres imprimés permet, à travers les médias, les événements publics, la présence en librairie, etc., une diffusion certes plus locale, mais plus diversifiée, et peut atteindre un public plus hétérogène.

La tendance actuelle est à la généralisation de la publication des résultats de recherche en libre accès ${ }^{6}$ et le soutien à la publication d'ouvrages imprimés et commercialisés n'est déjà plus pris en considération par le Fonds national suisse de la recherche scientifique. Il n'est pas question de remettre en cause l'utilité de la publication en libre accès de certains textes scientifiques, ou de certains formats, mais il peut être intéressant de réfléchir à la pertinence de ce mode de publication pour tout type de publication issue de la recherche, pour tout type d'éditeur, pour tout type de domaine, pour tout type de lectorat, pour tout type de diffusion. La publication en libre accès, si elle n'interdit pas formellement la publication d'un livre papier, influence la commercialisation de celui-ci et nécessite 
de repenser la production, la diffusion et le financement des ouvrages scientifiques (papier et/ou au format électronique). «Un paysage diversifié s'installe progressivement car la mutation numérique n'engendre pas les mêmes réorganisations dans les différents champs scientifiques" (Chartron \& Schöpfel, 20I7: §24).

\section{Le type de publication}

Un article est-il lu de la même manière qu'un ouvrage? Est-on immergé de la même manière dans l'un que dans l'autre? Le format condensé d'un article implique certainement un mode de lecture différent de celui d'un ouvrage, forcément plus développé et plus détaillé, plus lent dans son exposition. On tend à lire en diagonale ou en séquences à l'écran et ce mode de lecture implique une perception cognitive différente d'une lecture linéaire.

En outre, si les frais de publication pour un article en libre accès, les APC (Article processing charges), s'élèvent déjà à plusieurs milliers de francs, quels sont, de fait, les BPC (Book processing charges)? Une publication en libre accès ne permettant pas de rentrées financières par le biais de ventes, la totalité des frais de production des ouvrages doit, de fait, être assumée par des financements publics ou des recherches de fonds privés en amont.

\section{Le type de maison d'édition}

L'open access s'est, entre autres, développé pour contrecarrer les coûts élevés d'abonnements de revues pratiqués par certaines éditeurs de publications académiques. Le fait que ces maisons d'édition touchent des subventions publiques pour la publication de périodiques qui sont ensuite vendus à des bibliothèques, elles aussi publiques (Chillier, 2017), est critiqué (et critiquable). Néanmoins, faut-il considérer toutes les maisons d'édition

6 Le FNS ne soutient les publications que si elles sont publiées au format numérique et en libre accès. Le FNS n'entre pas en matière pour les frais d'impression d'un ouvrage. Si un éditeur choisit d'imprimer un livre et de le commercialiser, l'ouvrage soutenu par le FNS devra être rendu disponible en libre accès simultanément. Le FNS soutient l'Initiative internationale OA2O20 et revendique «une politique globale d'Open Access pour toutes les publications». Consulté le I I.oI.20I 8 sur http://www.snf.ch/fr/leFNS/points-de-vue-politique-de-recherche/open-access/Pages/default.aspx 
de la même manière, et vouloir évincer le travail de transmission qu'elles effectuent sous prétexte que certaines, les plus puissantes certes, ont une approche essentiellement commerciale du travail éditorial?

Si l'on considère le paysage romand (voire même français) de sciences humaines et sociales, on s'aperçoit qu'il est constitué principalement de structures relativement modestes ou de presses universitaires (Chartron, 2016: §49-53). On ne fait guère fortune en publiant des ouvrages de sciences humaines. En Suisse romande, les salaires des éditrices et éditeurs, dans des structures privées notamment, de sciences humaines et sociales sont en général plus bas que ce que leur niveau de qualification pourrait leur offrir dans d'autres branches (Pahud, 2013).

\section{Le type de domaine}

Les sciences sociales - dans lesquelles des recherches qualitatives plutôt que quantitatives sont menées - ont un cycle de vie plus long et un rythme de déploiement plus lent que les sciences dures. Même si notre société contemporaine évolue à un rythme effréné, les mécanismes sociaux prennent du temps à se déployer, du temps à se déceler, du temps à s'analyser. La recherche peut aussi avoir besoin de pages pour être valorisée et d'une lecture approfondie pour s'appréhender.

S'il est important de publier des recherches sur des données qui soient toujours actuelles et pertinentes, les sciences humaines et sociales ne sont pas astreintes à la course à la découverte et à la publication en primeur de résultats de recherche.

En outre, le travail social, en particulier, est souvent lié à des réalités locales en lien avec une organisation, une législation ou des politiques nationales, régionales ou communales.

Le livre imprimé, dans les pages duquel on peut se replonger, n'est donc pas incompatible avec le déploiement des sciences humaines et sociales. Le soin apporté à l'objet livre, dans sa matérialité, participe également de l'ancrage de la recherche dans la cité.

\section{Le type de lectorat}

La question du ou de la destinataire d'un ouvrage issu d'une recherche en sciences humaines et sociales a déjà été évoquée en termes d'écriture, on peut aussi s'intéresser au lectorat en termes de diffusion. Si ce sont 
les pairs qui sont visés, alors oui, la publication en ligne en libre accès peut faire sens. Ce mode de diffusion correspond souvent au retour de la recherche scientifique vers elle-même. Mais les directives actuelles des instances de soutien à la recherche et les institutions universitaires visent toujours plus à une vulgarisation de la recherche, à rendre les milieux académiques moins fermés sur eux-mêmes, à s'insérer dans la cité. Or, les sites sur lesquels les textes scientifiques sont mis en ligne sont peu connus du public intéressé non universitaire. Le livre imprimé permet une visibilité en dehors du sérail académique.

Les chercheur.e.s sont encouragé.e.s à publier des textes scientifiques en ligne et souvent en anglais. De fait, on peut se demander qui sont les destinataires de telles publications. En publiant en anglais, ce ne sont pas les partenaires de proximité qui sont visés. Si la publication en anglais n'est pas sans conséquence sur la constitution du lectorat, elle a aussi des répercussions sur la production du texte lui-même. Soit une traductrice ou un traducteur doit être sollicité.e (ce qui augmente le temps de production et les frais), soit l'auteur.e pourra rédiger en anglais, mais rares sont celles et ceux qui seront aussi à l'aise dans cette langue que dans leur langue d'étude.

\section{Le type de diffusion}

Il ne suffit pas qu'un texte soit publié gratuitement en ligne pour que ce texte soit lisible et visible par un large public. La valorisation de la recherche passe encore par la valorisation effectuée par les éditrices et éditeurs lors de vernissages, de tables rondes, de discussions autour d'un livre, de lectures, de mise à disposition en librairie, lors de colloques internationaux ou locaux, de comptes rendus dans des revues, d'articles dans la presse écrite, voire même sur des sites ou blogs, ou d'émissions radio, etc. Les Editions ies promeuvent leur catalogue au sein de l'institution, mais également dans des espaces publics du quartier, lors des Sorties ies - au café des Recyclables ou au restaurant Un $\mathrm{R}$ de famille, par exemple - lors de présentations dans des librairies - à la Librairie du Boulevard - ou encore, lors de manifestations en lien avec le livre: Salon du livre de Genève, d'Alger, Salon du livre du travail social (Caen), etc. -, lors de manifestations thématiques - Festival du film et forum international sur les droits humains -, lors de congrès et colloques internationaux ou de journées d'étude en lien avec le travail social - AIFRIS, UNAFORIS, congrès de la Société suisse de sociologie, etc. 
Le «libre accès» équivaut à une gratuité de lecture, mais pas forcément à la gratuité pour la bibliothèque, ${ }^{7}$ et en tout cas pas pour l'instance de production. Le travail éditorial conséquent effectué sur les manuscrits issus de recherches en SHS apportant une meilleure accessibilité et diffusion des résultats doit être rétribué. Ce mode de diffusion implique une rémunération des coûts de production plus grande pour les instances universitaires. Cet aspect doit être pris en considération dans leur budget. De même pour les instances publiques de soutien à la recherche. Il faut parfois avoir aussi recours à des instances privées pour soutenir une publication. On dénote actuellement déjà des frais très élevés d'APC pratiqués par certaines revues de grands groupes. Ainsi, un article de Guillaume Chillier, paru dans Le Courrier le 9 février 20I7, rapportait les propos d'Olivier Legendre, conservateur numérique de l'Université de Clermont-Ferrand: "Tout comme les tarifs des abonnements avaient dérapé, les tarifs de l'OA [open access] dérapent». Eliminer la possibilité de vendre un ouvrage, c'est réduire la possibilité de le promouvoir, de le rendre accessible à un plus large public, de faire en sorte qu'une partie des frais de production soient remboursés par cet apport financier.

Il y a quelques années, d'aucun.e.s parlaient de la disparition du livre papier; or les milieux professionnels du livre semblent revenir sur cette vision et l'idée d'une cohabitation entre papier et format électronique se répand. La lecture numérique ne semble pas décoller autant qu'anticipé et elle semblait même stagner en 2016 dans les pays anglophones (là où elle est la plus importante), donnant ainsi raison à Umberto Ecco et JeanClaude Carrière: N'espérez pas vous débarrasser des livres (2009). Si l'émergence des publications électroniques, gratuites ou non, ne va pour l'heure ni remplacer ni éliminer les publications papier, elles présentent une possibilité supplémentaire de publication - propre à certains types ou formats de texte -, s'adressent à un public spécifique - bien que l'accessibilité technologique se veuille paradoxalement très large -, et, bien loin de simplifier le travail de production des ouvrages et le travail éditorial, le complexifient et l'augmentent (augmentant, de ce fait, les coûts de production...).

\section{Conclusion}

Lors d'une journée d'étude à l'Université de Genève, intitulée «L'Open access dans les carrières académiques " (I I novembre 20I6), Axel Marion,8 
exposait la nécessité de viser $80 \%$ de publications scientifiques en libre accès à l'horizon 2020 (I00\% en 2024) - tout en insérant des mesures transitoires adaptées au paysage éditorial suisse. Il évoquait comme «forme alternative de publication » celle de «recréer des Presses universitaires en Suisse, dignes de ce nom afin qu'on puisse avoir, en utilisant notre potentiel non seulement d'infrastructures mais aussi évidemment de qualité du personnel académique [...] On peut créer des outils de publication qui permettent de garder le contrôle [...] et en même temps, on a cette qualité assurée par du peer review [...]". Axel Marion soutenait cette démarche pour la publication en libre accès, mais ce soutien peut également s'entendre pour des publications imprimées. De tels projets impliquent une volonté institutionnelle marquée et une politique éditoriale solide et résolument ouverte vers la cité. En finançant sa maison d'édition depuis plus de quarante ans et en ayant repensé et mis en place un nouveau projet éditorial depuis quelque trois ans, c'est ce à quoi s'attelle la Haute école de travail social de Genève.

La diffusion de la recherche se modifie, les exigences par rapport à celleci aussi. La science, qu'elle soit dure, pure, exacte, humaine et/ou sociale, doit s'adresser au plus grand nombre et être «virtuellement» la plus accessible possible. Accessible en termes de visibilité, mais également en termes de lisibilité et d'intelligibilité. Dès lors, si des clés de compréhension de notre société pluraliste et multiculturelle, que l'on dit en crise, se doivent d'être exposées au plus grand nombre, des stratégies pour ne pas véhiculer des approches tronquées ou trop pointues des mécanismes sociaux complexes de notre environnement sont à favoriser. Prendre le temps d'exprimer, de comprendre, d'entrer dans la finesse de la réalité, des terrains, trouver les mots pour dire le monde et l'appréhender sont une manière de participer au projet démocratique. Voilà l'ambition qui peut être celle d'un service de publication d'une Haute école de travail social.

Comme le «temps» de Proust, le livre n'est pas complètement perdu, pour autant qu'on le réinvestisse, voire le réinvente afin que sa capacité

7 En octobre 20I7, la plateforme OpenEdition Books a lancé un projet de financement d'ouvrages en souscription par des bibliothèques, "OpenEdition Books Select, une collaboration entre éditeurs et bibliothèques pour promouvoir l'accès ouvert aux ouvrages académiques en langue française».

8 Responsable du domaine «Politique des hautes écoles» de Swissuniversities. Vidéo consultée le I4.02.2017 sur https://mediaserver.unige.ch/play/98072 
créatrice et son potentiel émancipateur puissent se déployer pleinement, en lui accordant le temps de la lecture.

\section{Références bibliographiques}

Battaglini, M., Eckmann, M., Hasdeu, I. \& Savelieff, P. (20I5). Roms en cité. Témoignages, participations et politiques publiques. Genève: Editions ies.

Chartron, G. (2017). Stratégie, politique et reformulation de l'open access. Revue française des sciences de l'information et de la communication [En ligne], 8. Consulté le I2.0I.20I8 sur http://journals.openedition.org/rfsic/I836; DOI: I0.4000/ rfsic. 1836

Chartron, G. \& Schöpfel, J. (20I7). Open access et Open science en débat. Revue française des sciences de l'information et de la communication [En ligne], I I. Consulté le I r.or.20I8 sur http://journals.openedition.org/rfsic/333I

Chillier, G. (2017, 9 février). La coûts cachés du libre accès. Le Courrier, 3.

Cicourel, A. (20I8). La justice des mineurs au quotidien de ses services. Traduction de Samuel Bordreuil. Genève: Editions ies.

Carrière, J.-C. \& Ecco, U. (2009). N'espérez pas vous débarrasser des liures. Paris: Grasset.

Eckmann, M. \& Földhazi, À. (20I3). Articuler diversité et genre. Un défi pour les hautes écoles. Genève: Editions ies.

Mirénowicz, A. (2016, 2I avril). Bibliothèque: papier ou numérique? Hebdo.ch. Consulté le I5.09.17 sur http://www.hebdo.ch/hebdo/culture/detail/ biblioth\%C3\%A8que-des-lecteurs-et-des-livres.

Pahud, C. (2013). Editer... c'est imprimer? Pourquoi, au fond, publier des ouvrages de sciences sociales? Bulletin de la Société suisse de sociologie, I44, 9- I2.

Parazelli, M. \& Ruelland, I. (2017). Autorité et gestion de l'intervention sociale. Entre servitude et actepouvoir. Genève: Editions ies. 


\title{
Recherche en contexteS
}

\author{
Les apports du travail social aux sciences sociales
}

\section{Postface d'Eva Nada ${ }^{1}$}

"Je me suis efforcé de décrire le monde, non pas comme il est

mais comme il est quand je m'y ajoute, ce qui, évidemment, ne le simplifie pas» (Giono, 1953: 57)

Cet ouvrage collectif ${ }^{2}$ est parti d'une double volonté, à la fois historique et théorique: célébrer les 50 ans du Centre de recherche de la Haute école de travail social (CERES) et réfléchir aux apports de la recherche pour penser le travail social dans la cité et la formation. De manière plus générale, cet ouvrage entend également contribuer à la réflexion autour de la place des hautes écoles spécialisées dans le champ de la recherche, leurs apports et leurs liens avec les instances de financement tout comme avec les

1 Sociologue et adjointe scientifique à la Haute école de travail social de Genève depuis 20I2, j'écris cette postface à partir des différents rôles que j'ai occupés et occupe dans cette école. En 20I I, j'ai tout d'abord été engagée en tant que chercheure pour une enquête sur les centres éducatifs fermés, qui fait l'objet d'un article dans cet ouvrage, puis entre 2012 et 2017, en tant qu'adjointe scientifique au CERES, dirigé par Laurence Ossipow. Depuis 20I2, j'enseigne aussi en formation de base dans le cadre de cours sur la jeunesse, la méthode de recherche pour le travail de Bachelor et la sociologie de la pauvreté et des inégalités sociales. J'accompagne également, en tant que responsable de formation pratique (RFP), un groupe d'étudiantes de la volée 20I6. En tant que chercheure en doctorat, j'ai bénéficié d'une bourse de la relève HES-SO me permettant de me consacrer à ma thèse. Ces rôles variés m'ont fait voir l'institution sous différents angles, en expérimentant les enjeux propres aux missions de la HETS depuis la place du corps intermédiaire. En ce sens, la manière dont j'aborde et je traite théoriquement des apports du travail social aux sciences sociales est informée par ma pratique de l'institution et mes pratiques dans l'institution ainsi que mes pratiques de recherche.

2 Je remercie Monica Battaglini, Stéphanie Fretz et Laurence Ossipow pour leurs commentaires et remarques sur une version précédente de ce texte. 
universités. Dans cette visée, les dix contributions qui nous sont données à lire ont exploré les apports de la recherche pour l'étude du travail social selon deux axes: ses liens avec la cité et ses relations avec l'enseignement, soulevant des questions autour de la production, de la réception et des modes de diffusion de la recherche. A ce titre, les façons variées et diverses dont les thématiques du travail social sont abordées et les disciplines mobilisées, voire articulées, dans ces dix contributions interrogent certains schémas épistémologiques classiques des sciences sociales, desquels trois thématiques transversales émergent: I) le pluralisme des sciences ou les limites des frontières disciplinaires; 2) le rapport entre différents types de recherche ou le dépassement de l'opposition entre recherche fondamentale et appliquée; et enfin 3) l'implication du ou de la chercheur.e dans la production de connaissance ou la pluralité épistémique des sciences sociales et le savoir situé comme mode de production. Pour terminer ce voyage à travers la recherche en travail social dans les hautes écoles spécialisées, cette contribution vise donc à retourner le miroir en interrogeant non plus l'apport de la recherche au travail social comme objet, mais ce que le travail social apporte aux sciences sociales en dénouant les trois fils épistémologiques susmentionnés. En d'autres termes, il s'agit de s'interroger sur le fait que les sciences sociales se trouvent bousculées dans leurs sources, leurs cadres, leurs grilles d'analyses et leurs concepts théoriques par l'étude du travail social. A ce titre, nous discuterons du travail social non pas seulement comme objet sur lequel portent les recherches, comme savoirs des professionnel.le.s ou comme savoir des usagères et usagers, mais aussi comme sujet, c'est-àdire comme perspective depuis laquelle la connaissance se produit en prenant en compte ces différentes formes de savoirs. Cette postface vise ainsi à contribuer au débat autour des savoirs du travail social, leurs différents types et leur articulation, tout comme leur mutualisation.

\section{Etudier les multiples facettes du travail social: varier les disciplines, les approches, les méthodes d'enquête et les échelles d'analyse}

Les dix contributions de cet ouvrage illustrent comment le travail social est tout d'abord un objet constitué de multiples facettes mobilisant différentes approches, méthodes et disciplines pour l'appréhender. A cet égard, le travail social comme domaine d'enquête interroge le savoir disciplinaire et la prééminence de l'une ou l'autre des disciplines et approches pour son 
étude. Dans leurs chapitres sur le lien entre recherche et formation, Francis Loser, Manon Masse, Sylvie Mezzena et Kim Stroumza, Laurence Ossipow, Sophie Rodari et Laurence Bachmann montrent comment les différentes disciplines enseignées peuvent être conjointement mobilisées dans un enseignement autour d'un thème spécifique, mais aussi dans des modules thématiques. Appréhender son enseignement à partir d'un programme multidisciplinaire permet, comme le rappelle Laurence Ossipow, de susciter la réflexion et la réflexivité des étudiant·e•s. De son côté, Francis Loser propose, dans son enseignement sur la notion de posture professionnelle, d'aborder celle-ci à partir des différentes perspectives que cette notion ouvre, soit une perspective psychologique, sociale, éthique et esthétique. Mêlant des approches méthodologiques, variant les positionnements de la chercheure, des professionnel.le.s du travail social et des étudiant·e.s, Manon Masse fait dialoguer différentes méthodes et modes de production du savoir, de sa transmission et de sa réception. En s'inspirant de leurs expériences de recherche sur le rapport à l'argent des AS, Sophie Rodari et Laurence Bachmann soutiennent, de leur côté, la nécessité d'une approche plurielle des problématiques. Pour ces deux contributrices, cette approche plurielle, que cela soit dans l'enseignement de base, dans la formation continue ou dans leurs différentes pratiques de la recherche, doit être portée par des profils professionnels variés et spécifiques, soit, dans leur cas, une chercheure ayant une expertise de terrain et de ses méthodologies propres, et une chercheure bénéficiant d'une expérience de recherche d' 'excellence académique». Les deux chercheures soulignent l'importance de la pluralité des profils et de leur collaboration pour une circularité des savoirs entre la formation en travail social, la recherche sur le travail social et les institutions du travail social.

Les contributions de Claudio Bolzman, Monica Battaglini et Laurent Wicht, Arnaud Frauenfelder, Géraldine Bugnon et Eva Nada, et celle de Théogène-Octave Gakuba se situent chacune dans une approche disciplinaire et une approche méthodologique particulières. Au contraire des contributions autour du lien entre enseignement et recherche, les textes traitant du lien entre recherche et cité font moins dialoguer différentes perspectives et disciplines, dans la mesure où elles rendent compte des recherches menées dans des approches théoriques distinctes. Néanmoins, lues ensemble, les analyses menées à partir de perspectives différentes illustrent que le travail social comme objet d'étude implique de recourir à différentes disciplines pour en éclairer les différentes facettes et enjeux. A 
ce titre, et de manière plus générale, ces contributions participent au débat autour de la reconnaissance du travail social comme discipline, tout comme son inscription disciplinaire plurielle, qui traverse le champ professionnel du travail social et le champ académique en France (Rullac, 20I I) et en Europe (Rullac, Tabin \& Frauenfelder, 20I8). Toutefois, plutôt que de déterminer quelles seraient les conditions de constitution d'une discipline du travail social distincte et autonome des sciences sociales, ces différentes contributions sur l'étude du travail social par différentes disciplines - sociologie, psychologie, linguistique, anthropologie, sciences de l'éducation - appellent une interrogation épistémologique autour de la place des disciplines et de leur pluralisme dans la production de la connaissance en travail social.

Dans un ouvrage récent, Léo Coutellec (2015) rappelle que ce questionnement sur la pluralité des sciences traverse la philosophie des sciences depuis la fin des années I970, interrogeant la construction d'une pensée du pluralisme dans la science. Léo Coutellec propose d'appréhender la question de la pluralité comme un principe épistémologique ou une méthode qui repose sur l'idée d'une science qui ne se fonde pas sur des logiques disciplinaires, mais sur une logique de l'objet. Cette logique appelle les disciplines par fragments, c'est-à-dire que «les disciplines sont convoquées par les objets» en fonction de leur pertinence à les comprendre. Ce n'est plus la discipline qui commande les objets, qui les crée et qui détermine la manière d'agencer les savoirs. Cette «logique de l'objet» est une des conditions de la diversité épistémique, c'est-à-dire la possibilité d'accueillir des hypothèses, des approches et des connaissances alternatives (Coutellec, 20I5). Il ne s'agit toutefois pas de défendre une posture de «laisser-faire» ou une pensée du relativisme, mais de définir certains critères de la diversité épistémique, qui peuvent être variés et définis en contexte, influençant à chaque fois le contenu à donner à cette diversité tout en s'assurant que «les facteurs choisis de la diversité épistémique maintiennent la dissidence et suscitent la créativité» (Solomon, cité par Coutellec, 2015: 70). Dans cette optique, le travail social comme objet détermine et définit les approches et les «fragments de disciplines» les plus pertinents pour éclairer ses différentes facettes et répondre à ses enjeux. L'ensemble des contributions de cet ouvrage illustre comment la variété des méthodes d'analyse et des disciplines permet d'éclairer les multiples facettes et problématiques du travail social ainsi que de rendre visible des populations, des problèmes ou des enjeux impensés, qu'une discipline unique ne permettrait pas de faire, même si, historiquement, la sociologie en tant que discipline entretient 
une relation forte avec le travail social, dont elle fait un objet d'étude privilégié ${ }^{3}$ (Gutknecht, 2016; Ion, 2005; Ravon, 2003).

Dès lors, il ne s'agit pas d'enquêter sur le travail social, mais à partir du travail social, ce qui permet de déterminer quelles sont les approches, les méthodes ou les perspectives les plus heuristiques dans une situation donnée, dans un contexte spécifique et avec des actrices et acteurs particuliers. Dans cette optique, enquêter et enseigner à partir du travail social, et non plus sur le travail social, peut constituer une perspective et une problématisation qui s'autonomisent, en soumettant en retour les disciplines des sciences sociales et les concepts mobilisés à un regard critique pour penser le travail social comme domaine qui peut «se considérer théoriquement pour lui-même et surtout par lui-même» (Rullac, 20I I : 89). Il nous semble ainsi que cette approche épistémologique, qui pense d'abord la production de connaissance en termes d'objet plutôt que de discipline, s'avère heuristique pour le travail social, défini comme "des incertitudes à traiter et nécessitant une approche plurielle quant à leur compréhension" (Gutknecht, 20I6: I94), et cela tant pour les chercheur·e·s et les professionnel.le·s du travail social que pour les usagères et usagers. Ainsi, le rapport entre "savoir et pouvoir» interroge également les différents modes de production de la connaissance en travail social, ce qui se retrouve dans les différentes contributions, notamment à travers les débats qu'engagent les distinctions établies entre «recherche fondamentale et recherche appliquée» que nous discutons dans la section suivante.

\section{La recherche en travail social: savoir situé, savoir impliqué}

Comme le rappelle Claude de Jonckheere (20I6), le travail social entretient une relation intrinsèque avec la notion de problème: problème social, problème d'intervention, pris dans les problèmes que le travail social cherche à résoudre et dans les problèmes des sujets que ce dernier accompagne. A ce titre, la recherche en travail social est toujours une recherche située. En effet, elle s'inscrit dans des problèmes particuliers liés au contexte et au territoire tout comme dans des relations entre les chercheur.e.s, les

3 Ces liens forts et cette relation privilégiée avec le travail social trouvent leur origine dans la construction de la sociologie comme discipline (Berthelot, 20I4). 
professionnel.le.s du travail social et les populations concernées par l'intervention sociale, comme nous le rappellent différentes contributions de cet ouvrage. Les contributrices et contributeurs recourent à des concepts sociologiques ou anthropologiques qu'elles et ils s'attellent à interroger et à revoir à l'aune du contexte dans lequel les recherches sont menées, ainsi que le rappellent Arnaud Frauenfelder, Géraldine Bugon et Eva Nada dans leur étude sur les centres éducatifs fermés en Suisse, Monica Battaglini et Laurent Wicht sur la notion de mixité sociale, ou encore Sylvie Mezzena et Kim Stroumza par leur approche pragmatique. Etudier le travail social engage alors les chercheur.e.s à emprunter une perspective située et historicisée sur son objet. Ces conditions de production des connaissances en travail social les amènent à interroger la différenciation, voire la hiérarchisation, entre «recherche appliquée et fondamentale». Comme cela a été évoqué dans certaines des contributions, cette différence entre les deux types de recherche est le propre de la distinction établie entre les hautes écoles spécialisées et les universités. Elle prend toutefois des formes particulières en travail social, dans la mesure où la recherche se fait non pas seulement sur des phénomènes ou problèmes sociaux, mais sur des personnes qui agissent sur ces dernières, de même que sur les usagères et usagers qui sont affectés par ces problèmes ou phénomènes sociaux.

Dans sa contribution, Claudio Bolzman rappelle cette condition de savoir situé de la recherche en travail social, préférant à l'opposition entre recherche fondamentale et recherche appliquée la notion de recherche collaborative, en tant que recherche à la fois fondamentale et appliquée dont les aproches se complètent. La recherche collaborative se co-construit entre les partenaires concernés et joue sur les deux registres de production de connaissance, soit la recherche fondamentale et les «besoins du terrain". A travers les travaux menés au cours de sa carrière, Claudio Bolzman conclut que la condition même de la recherche en travail social, quelles qu'en soient les modalités, est le souci de son articulation avec les pratiques professionnelles. Il ne s'agit plus tant d'interroger la hiérarchisation entre ces deux formes de recherche que leur articulation. exprimée sous la forme d'un continuum que l'on retrouve dans certaines des contributions de cet ouvrage.

Ce questionnement autour de l'articulation des différentes formes de recherche traverse les débats sur les formes de savoir du travail social. Il est possible de distinguer trois types de savoir en travail social, qui se déclinent selon leur fonction et leur degré de scientificité (Gutknecht, 2016: I28-133): I) un savoir élaboré dans une visée d'intervention sociale, qui repose sur 
des concepts généralement issus des sciences humaines. Ce savoir pratique se construit en vue d'une intervention, autour de laquelle se cristallisent des discussions sur sa scientificité et la constitution du travail social comme discipline; 2) un savoir sur le travail social, qui vise à analyser son fonctionnement, ses dispositifs, l'activité professionnelle dans une optique de description, d'identification, de mise en discussion ou d'une perspective critique du travail social. Ce savoir est considéré comme le plus scientifique des trois, dans la mesure où il s'inscrit dans une discipline établie, soit la sociologie; 3) un savoir pratique élaboré par les professionnel.le.s face à une situation spécifique. Ce savoir est considéré comme ayant probablement le degré de scientificité le plus bas. Stimulante pour réfléchir aux savoirs du travail social, à leur articulation et à leurs effets, cette typologie reste néanmoins structurée par la notion de scientificité du savoir, celle-ci renvoyant à la plus ou moins grande extériorité de la démarche de la ou du chercheur.e (Tabin, 20I4), conception qui se retrouve en partie dans la notion de continuum.

Néanmoins, ce continuum entre recherche fondamentale en tant que condition de production de connaissance par son indépendance et recherche appliquée en tant que réponse aux «besoins des terrains » est de plus en plus interrogé par les épistémologies des sciences sociales, notamment dans ce qu'Elsa Dorlin et Annie Bidet-Mordrel (2009) nomment les épistémologies de la domination, mais aussi plus largement au sein de la philosophie et de l'épistémologie des sciences. Ainsi, Léo Coutellec (20I5) défend une épistémologie des sciences impliquées, c'est-à-dire visant le dépassement des catégories de science appliquée et de science expliquée (autre manière de définir une science fondamentale) pour créer entre elles du sens commun. L'épistémologie d'une science impliquée vise à la reconnaître, non pas comme neutre et autonome, mais comme « une science qui tient sa pertinence à sa tolérance au pluralisme et aux valeurs et donc qui abandonne son idéal d'autonomie et de neutralité sans abandonner son exigence d'impartialité" (Coutellec, 2015: 39). Ainsi, pour l'auteur, le fait que le savoir soit impliqué dans un contexte et médiatisé par des valeurs ou des intentions n'est pas un problème en soi, mais le devient quand ces éléments ne sont pas reconnus. L'auteur conçoit donc l'impartialité - notre rapport au réel et les procédures de vérification - comme impliquée, c'està-dire «comme étant prise dans un contexte et contrainte dans un jeu de valeurs" (Coutellec, 20I5: 42) qu'il s'agit de reconnaître comme condition de production de connaissance. 
Etudier le travail social sous l'angle de différents phénomènes sociaux, tels que la mixité sociale, le rapport à l'argent, la situation de handicap, la maltraitance institutionnelle ou le don met en évidence que le travail social n'est pas seulement un domaine d'enquête, mais peut aussi être une catégorie d'analyse des rapports sociaux. Toutes les contributions n'appréhendent pas forcément leur objet en ces termes. Néanmoins, en s'intéressant à des thèmes comme le handicap (Loser), la maltraitance institutionnelle (Masse), les personnes âgées migrantes (Bolzman), l'enfermement des jeunes (Frauenfelder, Bugnon \& Nada), le rapport à l'argent des AS (Rodari \& Bachmann), la question de la «différence» sociale des populations concernées par ces problématiques rend également visibles des formes de hiérarchisation, de normes et valeurs dominantes, ainsi que des rapports de pouvoir. A ce titre, les chercheur.e.s dans le domaine du travail social sont pris dans et participent au rapport de pouvoir et de savoir, ce qui interroge leur positionnement à l'égard de celui-ci et de ses objets.

A cet égard, nous défendons l'idée que le travail social est plus qu'un objet d'étude: il doit aussi être envisagé comme une perspective, n'ouvrant pas seulement sur l'historicisation de l'objet d'étude et la production de savoirs situés, mais aussi sur l'implication des chercheur.e.s à l'encontre de leur objet d'étude. Comme l'illustrent certaines contributions de cet ouvrage, le savoir du travail social impose aux chercheur.e.s de revoir leur positionnement, leur mode de production de connaissance et de restitution de celle-ci, en impliquant les professionnel-le-s ainsi que les usagères et usagers dans le processus en tant que sujets porteurs d'un savoir - et non pas seulement comme objet de savoir - ainsi que de l'importance de ce savoir dans le travail social notamment ${ }^{4}$ (Gutknecht, 20I6). Ce propos souligne à quel point l'articulation des différents types de savoir du travail social, et plus généralement le travail social comme objet, vient bousculer les frontières entre "recherche appliquée et recherche fondamentale» et redéfinit les objets de recherche et les processus de restitution.

\section{Vers une pluralité épistémique de la recherche en travail social}

Les enjeux soulevés autour de la différenciation établie entre recherche fondamentale et recherche appliquée posent frontalement la question des manières de considérer les savoirs, de les différencier et de les hiérarchiser. Si cette tension est exacerbée dans le champ du travail social, c'est qu'elle 
est constitutive de ce même travail social. En effet, si le travail social se charge des questions sociales d'une société, c'est à partir de ses marges qu'il le fait, d'où son aporie; il doit prendre en charge les questions sociales en les rendant invisibles, en les laissant dans l'ombre pour qu'elles ne viennent pas perturber l'ordre social (Castel, I995). Dans ce contexte, faire de la recherche en travail social permet de rendre visible des objets et des phénomènes sociaux qui restent par nature dans la marge tout comme le savoir des professionnel.le.s du travail social, et des usagères et usagers. Si la recherche permet de visibiliser ce savoir, il peut également amener les chercheur.e.s à revoir leur objet, leur méthode, voire à intégrer les actrices et acteurs comme sujet connaissant, à l'instar de ce que montre la contribution de Kim Stroumza et Sylvie Mezzena. Dans ce sens, le travail social comme objet à la marge et peu visible oblige les chercheur.e.s - productrices et producteurs d'une connaissance située et locale, donc d'un pouvoir -, à se poser la question de la pluralité épistémique, c'est-à-dire de la place accordée aux différents points de vue dans la production du savoir.

Dans sa contribution, Théogène-Octave Gakuba se confronte et affronte cette pluralité en enquêtant auprès de jeunes avec lesquels il partage la même origine. Ces jeunes, partageant leur expérience de migration et d'exil avec lui, le conduisent à se positionner face à ce que la démarche d'entretien produit en termes d'attentes et de reconnaissance. Il est ainsi amené à devoir, non seulement porter attention à leurs points de vue, mais aussi à leur accorder le privilège épistémique, c'est-à-dire à reconnaître la pertinence de leur connaissance située et construite à partir de leurs expériences et position marginales, en saisissant toutes les occasions qui lui sont offertes - par sa position de chercheur - pour faire reconnaître leurs points de vue et transformer l'expression de leurs expériences en savoir (Haraway, 2007; Harding, I99I). Cette confrontation à l'expérience et au savoir des jeunes l'amène à revoir les principes de l'objectivation ou de la neutralité axiologique et à devoir se positionner face aux jeunes et à leurs expériences, de même que dans la transmission de ce savoir dans des arènes publiques. Cette contribution soulève, de fait, la question de la validité des savoirs produits, en tant que savoirs situés. Prendre en considération le point de vue et le savoir minoritaires entendus comme

4 Pour approfondir le sujet, nous renvoyons la lectrice ou le lecteur aux pages I9II98 de l'ouvrage de Thierry Gutknecht (2016), Actualité de Foucault: une problématisation du travail social, et plus généralement à l'ensemble du chapitre IV. 
émanant de celles et ceux qui n'ont pas le privilège épistémique dans la production du savoir renvoie au concept d'objectivité forte (Harding, I99I). Ce concept a deux principes: un principe d'étrangeté (partir des positions minoritaires qui n'ont pas le privilège épistémique) et un principe de réflexivité (processus d'objectivation du sujet connaissant).

Dans son article, Francis Loser relève la nécessité de se positionner comme sujet connaissant, tant pour le ou la chercheur.e que pour le ou la professionnel.le du social. Il montre que les expériences de mise en situation dans le processus de recherche et dans l'enseignement s'avèrent un mode heuristique pour prendre conscience de son positionnement et des processus de stigmatisation à l'égard des personnes en situation de handicap, tout comme de son propre privilège de personne valide. Toutefois, en ne considérant que la position du sujet connaissant, le risque est de ne penser les personnes en situation de handicap que comme des victimes et de gommer le principe de privilège épistémique, c'est-à-dire la connaissance des personnes en situation de handicap. En effet, selon le contexte et le point de vue adoptés sur l'objet, le privilège n'est pas toujours dans ce qui apparaît au premier regard comme évident, soit, ici, la personne reconnue comme valide physiquement ou mentalement. En fait, dans des conditions matérielles particulières, le «stigmate» peut devenir un privilège dans le rapport à soi et au monde qu'il permet, comme nous le montre le film Touch me not (Pintilie, 20I8). ${ }^{5}$ En interrogeant la question de l'intimité à travers le rapport au toucher (au sens propre et figuré) entre personnes de différents horizons et origines sociales, le film documentaire montre que les personnes en situation de handicap peuvent être en situation d'avantage et non de handicap. En effet, les différent.e.s protagonistes du film qui ne sont pas en situation de handicap physique semblent nettement moins à l'aise avec leur corps, dans leur rapport à l'autre et avec ce qui s'exprime à travers le sens du toucher, que la personne physiquement et gravement handicapée. Elles entretiennent un rapport à elles-mêmes, à leurs corps et aux autres qui leur ouvre un champ des possibles conduisant les «autres» à redéfinir leur regard pour les amener à revisiter leur rapport à elles-mêmes et leur rapport au monde.

Ce principe de pluralité épistémique habite les productions et l'organisation des collections des Editions ies. Le désir de partager les savoirs produits selon des positions et des cadres conceptuels variés caractérise cette maison d'édition. L'enjeu réside alors dans un savant mélange entre rendre compte de formes de savoir produites à partir de positions et points de vue différents, sans céder à une hiérarchisation de ces productions ou à un relativisme. 
Ainsi, comme le relève Stéphanie Fretz dans sa contribution, les différentes collections des Editions ies visent à rendre accessibles et visibles, non pas seulement des recherches propres aux sciences sociales - notamment à travers la traduction d'ouvrages classiques des sciences sociales-, mais aussi le savoir issu des pratiques des professionnel.le·s, tout comme des recherches menées dans une perspective de travail social par des chercheur.e.s établi.e.s ou par des étudiant·e.s lors de leurs travaux de bachelor.

La collection "Pratique.s " ${ }^{6}$ donne à voir des praticen.ne.s du travail social qui ne sont plus seulement un objet de connaissance, mais des sujets participant à produire du savoir, en inventant des nouvelles formes de langage et de partage. Le dernier ouvrage publié, Traceurs de chemin (Hauser \& Michaud, 2018), en est une belle illustration. Yolande Hauser, éducatrice de la petite enfance, partage ses expériences de travail et son savoir pédagogique, et rend compte par son parcours professionnel des transformations de la pratique du métier d'éducatrice et d'éducateur ainsi que de ses implications en termes pédagogiques. Elle et son co-auteur, Stéphane Michaud, maître d'enseignement à la HETS et pédagogue, ont ajouté des encadrés, distinguant ainsi deux formes de langage au sein d'un même texte, posant véritablement "des arrêts sur image et des formes réflexives" issus de leur dialogue. ${ }^{7}$ Ainsi, Yolande Hauser et Stéphane Michaud ne tracent pas seulement les chemins d'une pratique pédagogique, mais sont aussi traceuse et traceur de chemin de nouvelles formes de langage et de partage du savoir articulant recherches, expériences, concepts et réflexivités. Si les enjeux de distinction des rôles et des savoirs sont visibles dans la mise en forme du texte, donnant parfois le sentiment d'une différenciation et d'une hiérarchisation du savoir, la publication prend le risque de s'ouvrir aux questionnements d'une pluralité d'actrices et d'acteurs et

5 Film de la cinéaste roumaine Andina Pintille qui a obtenu l'Ours d'Or au Festival du cinéma de Berlin en 2018.

6 La collection «Pratique.s» se veut être un lieu d'expression pour les professionnel.le.s, les formatrices et formateurs ainsi que pour les étudiant.e.s.

Pour illustrer l'organisation du livre, prenons comme exemple le chapitre 2, «Tracer le chemin ", qui raconte la construction d'un jardin d'enfants, le Chat botté, de son agencement intérieur à la constitution d'une équipe en passant par la mise en place d'une pédagogie. Dans cette histoire s'intercalent des encadrés renvoyant à des notions plus théoriques et transversales à la pratique pédagogique, comme «construire une posture professionnelle» ou «le concept de médiation dans la petite enfance». 
"à d'autres processus de légitimation tout aussi importants et pas plus faciles", comme le rappelle Coutellec (2015: 72). A ce titre, Kim Stroumza et Sylvie Mezzana, par la publication d'un DVD comme objet hybride - de formation et de transmission de savoir -, prennent aussi le risque de s'ouvrir à d'autres processus de légitimation, avec des enjeux scientifiques pour les chercheures dans la possibilité de transformer leur propos dans un langage visuel et pédagogique, mais aussi dans un langage professionnel pour les éducatrices, les politiques et les responsables d'institution. Enfin, comme le montrent, chacune à leur manière, les contributions de Laurence Ossipow et Manon Masse, la pratique de l'enseignement, en dialoguant avec les étudiant.e.s, peut amener d'une part les chercheur.e.s à revoir leurs concepts, mais aussi à considérer le savoir des étudiant.e.s en lui-même et à le valoriser par la publication ou la formation. ${ }^{8}$

Pour conclure cette discussion, il nous faut rappeler que le CERES est né en I968, pourrait-on dire, de cette ambition d'inventer de nouvelles formes de légitimité épistémique et sociale, en souhaitant valoriser et diffuser les travaux de recherche des étudiant·e.s, soit des travaux produits à partir de positions minoritaires, auxquelles est reconnu par là un privilège épistémique au même titre que celui des enseignant.e.s ou des chercheur.e.s. En ce sens, le CERES est peut-être précurseur de ce que Léo Coutellec appelle de ses vœux comme une épistémologie des sciences impliquées. La recherche, la formation et la publication à la HETS rappellent ainsi la nécessité d'un savoir situé, produit dans un contexte spécifique, visant non pas seulement à valoriser et publiciser les recherches des chercheur·e·s, mais aussi différents types de savoirs émanant des professionnel.le.s de l'intervention et des usagères et usagers ou encore des étudiant.e.s.

\section{Vers une perspective de travail social comme condition d'une science démocratique}

«Malinowski alla aux îles Trobriand et étudia des populations qu'il n'aurait probablement pas aimé inviter à dîner; Radcliffe-Brown étudia les habitants des îles Andaman qui mangeaient du cochon cuit à la broche - cela s'appelle l'anthropologie, l'étude de l'homme. Lorsque nous nous sommes mis enfin à étudier les populations qui se trouvaient ici, dans nos propres villes, nous avons appelé cela la «sociologie», l'étude de nos concitoyens. Dans les deux cas, notre but n'était pas d'étudier les populations qui sont les plus proches. 
Nous continuons à étudier les populations qui sont relativement défavorisées. En pratique, nous gardons l'idée qu'il y a ceux qui ont un mandat pour faire des études, et ceux dont le destin est d'être étudiés afin d'être préservés comme les aborigènes, ou afin d'être éclairés et réhabilités. Mais, que nous nous nommions anthropologues ou sociologues, nous nous acheminons vers l'égalité avec ceux qui sont les objets ou les sujets de nos études. Nous pourrons un jour n'étudier que nos égaux, c'est-à-dire des gens qui pourraient très bien nous étudier» (Hughes, 2004 [1974]: 314-3I5)

Comme cet extrait d'Everett Hughes nous le rappelle ici, les disciplines de l'anthropologie et de la sociologie se sont construites sur un rapport de pouvoir et de disciplinarisation des observant.e.s sur les observé.e.s. Dans cette perspective, le travail social a souvent été étudié comme une entreprise de normalisation des personnes défavorisées, telles que, par exemple, les classes populaires (Donzelot, I977; Muel-Dreyfus, I983; Frauenfelder, 2016). Ces travaux historiques et sociologiques ont participé à produire un savoir sur le travail social qui permet l'historicisation du pouvoir du travail social et la conscientisation de celui-ci. Sans nier les dimensions de contrôle social et de normalisation du travail social, la conscience de son pouvoir s'accompagne aussi d'un savoir sur la société et de sa problématisation permettant à de nouvelles pensées et modes d'action d'émerger, comme nous le rappellent Claude de Jonckheere et Thierry Gutknecht (2016), et comme l'illustrent notamment les contributions de Stéphanie Fretz et de Théogène-Octave Gakuba dans le présent ouvrage. ${ }^{9}$ En tirant des dix

8 Dans une approche voisine, l'ouvrage de Nicolas Jounin (20I4) Voyage de classes relate l'enseignement méthodologique de recherche donné à des étudiant.e.s de sociologie de l'Université de Saint-Denis, Paris 8. Le but de son enseignement est de permettre aux étudiant.e.s issu.e.s d'un milieu social populaire de faire une expérience de recherche, et cela dans un milieu social éloigné du leur, ici les beaux quartiers parisiens. Cette expérience d'enseignement et de recherche est également portée par un souci de démocratisation, non pas seulement d'accès au savoir, mais aussi à sa production, en montrant que celles et ceux qui sont généralement objets d'étude, jeunes issus de milieux populaires, peuvent aussi étudier celles et ceux qui les étudient habituellement.

Sur ce point, nous renvoyons aux travaux de Shirley Roy (20I4), notamment à l'article «Entre terrain et monde universitaire: les défis du partenariat», ou encore à l'ouvrage Le travail social à la recherche de nouveaux paradigmes (Tschopp, Libois \& Bolzman, 2013), qui discute également ces questions. 
contributions de cet ouvrage trois fils épistémologiques - le pluralisme des sciences, le rapport entre différents types de recherche et la pluralité épistémique des sciences sociales - nous avons essayé de décrire ce qu'emprunter une perspective du travail social sur les problèmes d'une société implique en termes de positionnement et de recherche pour nous engager vers la possibilité d'une science démocratique, c'est-à-dire partageant une responsabilité épistémique dans la production des savoirs. L'enjeu est de donner les moyens à des populations qui sont généralement plutôt l'objet d'études d'être aussi productrices de connaissance et qu'elles puissent tout autant étudier des espaces éloignés de leur milieu social, ce qu'Everett Hughes appelait déjà de ses vœux dans les années I970. Tant l'objectif originel du CERES que le développement de la recherche en travail social vont dans ce sens. En retour, la confrontation à d'autres formes d'expériences, et donc de savoirs, bouscule les modes de production de la connaissance et remet en question la division du travail de recherche entre «recherche fondamentale et recherche appliquée», que celle-ci soit envisagée comme un continuum ou une dichotomie irréconciliable. A cet égard, entretenir les liens avec les usagères et usagers du travail social, les professionnel.le.s et les institutions sociales confère aux chercheur.e.s de la HETS une responsabilité à faire constamment retour sur leur positionnement, leurs outils, leurs objets de recherche et leurs pratiques. Plus encore, comme nous l'avons discuté tout au long de cette postface, ce positionnement particulier de la recherche en travail social implique de se confronter à la question de l'implication du ou de la chercheur.e par rapport à son objet d'étude, de son positionnement, de ses valeurs, de ce qui l'amène à choisir ce sujet (Jounin, 20I4), mais aussi de prendre en considération le savoir de celles et ceux pris pour objets d'enquête. Ainsi que le souligne Thierry Gutknecht, «ces entreprises de croisement de regards et des savoirs permettent un enrichissement des connaissances des différents acteurs, de nouvelles représentations de ce qu'est le savoir, et surtout donnent lieu à une meilleure compréhension des situations sur lesquelles interviennent les professionnels" (Gutknecht, 20I6: I3I). En ce sens, étudier le travail social oblige à enquêter à partir du travail social en tant que perspective, et non pas dans, sur ou avec.

Néanmoins, et pour conclure, une des craintes qui s'exprime sous différentes formes dans ces diverses contributions est le risque que les sciences sociales - en tant qu'elles sont constituées par différentes disciplines perdent leur autonomie comme champ académique, notamment en se voyant imposer les termes du débat scientifique et les modes de faire de la 
recherche à travers des enjeux économiques ou des réponses aux «besoins des terrains ". Ces enjeux amènent à cristalliser les positions des un.e.s et des autres autour de la défense de leur doxa et de leurs champs d'expertise. Pourtant, nous avons voulu montrer, à travers cette discussion, que l'enjeu nous semble moins de défendre ses frontières disciplinaires ou professionnel.le·s que de reconnaître la nécessité d'une pluralité épistémique comme mode de résistance et de défense de la sociologie, et plus largement des sciences sociales, ainsi que le propose Raewyn Connell, célèbre sociologue, dans son article In Praise of sociology (2017). S'inscrivant dans le contexte anglo-saxon, elle reconnaît que «si les managers et politiciennes et politiciens néolibéraux n'ont peut-être plus besoin de la sociologie, elle est en revanche vitale pour une société démocratique. Ce savoir est nécessaire pour informer le débat public et pour prendre des décisions collectives intelligentes [...] Aujourd'hui, d'autres idées puissantes émergent et l'étendue de la pensée sociale créative est immense. C'est ce type de sociologie dont nous avons besoin et elle peut être réalisée „10 (Connell, 20I7: 294295 [trad.]). Une perspective en travail social peut contribuer à cet objectif, dans le sens où elle s'avère heuristique comme mode de production de connaissance, comme méthode pour penser les différents types de savoir du travail social, et ce qu'ils apportent comme modes de compréhension du monde, et donc comme possibilités de changement.

Texte original: «Though sociology is not much needed by neoliberal managers and politicians, it is vital for a democratic society. Its knowledge is needed for informed public debate, and for intelligent collective decision making. [...] Yet other powerful ideas are emerging and there is enormous scope for creative social thought. That is the kind of sociology we need, and it can be made."

\section{Références bibliographiques}

Berthelot, J.-M. (20I4). La construction de la sociologie. Paris: Presses Universitaires de France.

Castel, R. (I995). Les métamorphoses de la question sociale: une chronique du salariat. Paris: Fayard.

Connell, R. (20I7). In Praise of Sociology. Canadian Review of Sociology/Revue canadienne de sociologie, 54(3), 28I-296.

Coutellec, L. (20I5). La science au pluriel. Essai d'épistémologie pour des sciences impliquées. Versailles: Quæ. 
Donzelot, J. (1977). La police des familles. Paris: Minuit.

Dorlin, E. \& Bidet-Mordrel, A. (Ed.). (2009). Sexe, race, classe: pour une épistémologie de la domination. Paris: Presses Universitaires de France.

Frauenfelder, A. (20I6). Le retour de l'«enfance en danger». Seuil de sensibilité, modes d'intervention et normes de parentalité en mutation. Dans B. Vittori (Ed.), Au risque de la prévention. Genève: Editions ies.

Ion, J. (Ed.). (2005). Le travail social en débat(s). Paris: La Découverte.

Giono, J. (1953). Voyage en Italie. Paris: Gallimard.

Gutknecht, T. (2016). Actualité de Foucault: une problématisation du travail social. Genève: Editions ies.

Haraway, D. (2007). Manifeste cyborg et autres essais. Sciences, fictions, féminismes. Anthologie établie par L. Allard, D. Gardey \& N. Magnan. Paris : Exils.

Harding, S. (I99I). Strong objectivity and socially situated knowledge. Dans S. Harding (Ed.), Whose science? Whose Knowledge? Ithaca, NY: Cornell University Press.

Hauser, Y. \& Michaud, S. (2018). Traceurs de chemin: parcours d'une pédagogue de la petite enfance. Genève: Editions ies.

Hughes, E. C. (2004). Le regard sociologique: essais choisis. Paris: EHESS.

Jonckheere, C. de (2016). Préface. Dans T. Gutknecht, Actualité de Foucault. Une problématisation du travail social (pp. I I-I7). Genève: Editions ies.

Jounin, N. (20I4). Voyage de classes: des étudiants de Seine-Saint-Denis enquêtent dans les beaux quartiers. Paris: La Découverte.

Muel-Dreyfuss, F. (1983). Le métier d'éducateur. Les instituteurs de I900. Les éducateurs spécialisés de 1968. Paris: Minuit.

Pintilie, A. (2018). Touch Me Not. Consulté le 10.05.I8 sur http://www.imdb.com/title/ tt4949 I I $2 /$

Ravon, B. (Ed.). (2003). Le travail social. Paris: La Documentation française.

Roy, S. (20I4). Entre terrain et monde universitaire: les défis du partenariat. Dans S. Voélin, M. Eser Davolio \& M. Lindenau (Ed.), Le travail social entre résistance et innovation/Soziale Arbeit zwischen Widerstand und Innovation (pp. I23-133). Genève/Lucerne: Editions ies/Interact Verlag.

Rullac, S. (20 I I). Le travail social et la science. VST - Vie sociale et traitements, Io9, 89-95.

Rullac, S., Tabin, J.-P. \& Frauenfelder, A. (2018). La fabrique du doctorat en travail social. Controverses et enjeux. Rennes: Presses de l'EHESP.

Tabin, J.-P. (20I4). Jalons épistémologiques de la recherche en travail social. Dans S. Voélin, M. Eser Davolio \& M. Lindenau (Ed.), Le travail social entre résistance et innovation/Soziale Arbeit zwischen Widerstand und Innovation (pp.349-358). Genève/Lucerne: Editions ies/Interact Verlag.

Tschopp, F., Libois, J., \& Bolzman, C. (Ed.). (2013). Le travail social à la recherche de nouveaux paradigmes: inégalités sociales et environnementales. Genève: Editions ies. 


\section{Les contributrices et contributeurs}

\section{Laurence Bachmann}

Sociologue, elle est professeure assistante à la Haute école de travail social (HESSO/Genève) et directrice du MAS-DAS en Direction d'institutions éducatives, sociales et socio-sanitaires de la HES-SO. Ses principaux domaines de recherche sont liés au processus de transformation de soi inhérent à l'acquisition d'une posture professionnelle et, dans ce cadre, à la question de l'appropriation des sciences sociales.

\section{Monica Battaglini}

Docteure en Sciences politiques, elle est professeure associée à la Haute école de travail social (HES-SO/Genève). Elle est spécialisée dans l'analyse des politiques publiques en lien avec les récentes transformations de l'Etat social et ses relations avec les actrices et acteurs de la société civile. Elle étudie l'impact de ces développements sur les actions publiques au niveau urbain, en Suisse particulièrement.

\section{Claudio Bolzman}

Sociologue, il est professeur ordinaire à la Haute école de travail social (HESSO/Genève). Ses travaux portent notamment sur les questions migratoires et les relations à l'altérité. Il est spécialiste de la thématique " âge et migration "sur laquelle il a mené diverses recherches, et a contribué à de nombreuses publications.

Géraldine Bugnon

Docteure en Sociologie, elle est adjointe scientifique à la Haute école de travail social (HES-SO/Genève). Elle s'intéresse aux diverses formes de régulation sociale et pénale des déviances juvéniles.

\section{Monique Eckmann}

Sociologue, elle est professeure honoraire de la Haute école de travail social (HESSO/Genève). Ses travaux portent sur les dialectiques d'inclusion et d'exclusion, les conflits intergroupes, les relations entre minorités et majorités, les questions d'identité et de mémoire, en particulier à propos de racismes, d'extrémismes, d'antisémitisme et autres formes de haine à l'encontre de groupes. 
Arnaud Frauenfelder

Sociologue, il est professeur ordinaire à la Haute école de travail social (HESSO/Genève) et responsable du Centre de recherche sociale (CERES). Il mène des recherches au croisement de la sociologie de la socialisation, de la jeunesse, du travail et des classes sociales, de la déviance et du contrôle social, et ce sur différents terrains (aide sociale, jardins ouvriers, naturalisation, prison, protection des mineur.e.s).

\section{Stéphanie Fretz}

Diplômée de Lettres modernes, elle est responsable du service des Editions ies de la Haute école de travail social (HES-SO/Genève). Elle exerce depuis plus de quinze ans dans le milieu éditorial universitaire. Elle entrevoit des similitudes entre son activité d'éditrice et celle d'une sage-femme.

\section{Théogène-Octave Gakuba}

Docteur en Sciences de l'éducation avec une spécialisation en Psychologie interculturelle et diplômé en sciences politiques et relations internationales, il est adjoint scientifique à la Haute école de travail social (HES-SO/Genève). Ses intérêts de recherche portent sur les aspects psychologiques de la migration et de l'exil, la résilience des personnes migrantes et réfugiées, les jeunes migrant·e.s, la qualité de vie des personnes migrantes âgées.

\section{Francis Loser}

Docteur en Sciences de l'éducation, diplômé en éducation sociale et en art-thérapie, il est professeur associé à la Haute école de travail social (HES-SO/Genève). Ses activités de recherche et d'enseignement s'intéressent aux pratiques situées à la croisée de différents champs - travail en réseau, médiation artistique en travail social, art et handicap, etc. - et à la professionnalité - récits professionnels, analyses des pratiques et supervision - selon une perspective pragmatique et esthétique.

\section{Manon Masse}

Docteure en Sciences de l'éducation et détentrice d'un DES en Psychologie clinique, elle est professeure associée à la Haute école de travail social (HES-SO/Genève) et au bénéfice d'une longue pratique de terrain auprès des personnes en situation de handicap et de leurs familles. Ses domaines de recherche portent sur la prévention de la maltraitance, l'autodétermination, l'empowerment et la participation sociale des personnes en situation de handicap.

\section{Sylvie Mezzena}

Docteure en Sciences de l'éducation, elle est professeure associée à la Haute école de travail social (HES-SO/Genève). Elle consacre ses recherches à la construction de la professionnalité dans l'expérience de l'activité ainsi que dans les dispositifs d'alternance dans les métiers de l'intervention sociale. Inscrits dans le courant de l'analyse de l'activité et dans un cadre théorique pragmatiste, ses travaux questionnent tout particulièrement la construction de la connaissance et des valeurs dans l'activité. 
Eva Nada

Chercheure en Sociologie, elle est adjointe scientifique à la Haute école de travail social (HES-SO/Genève). Elle s'intéresse aux modalités de prise en charge de la transition de l'école à l'emploi dans les dispositifs d'activation des politiques sociales, aux relations professionnelles dans le travail social et aux différentes expériences de la prise en charge des jeunes bénéficiaires.

Laurence Ossipow

Docteure ès Lettres et anthropologue, elle est professeure ordinaire à la Haute école de travail social (HES-SO/Genève). Après avoir travaillé sur la thématique de l'alimentation dite alternative ainsi que sur celles de l'immigration et de la citoyenneté, elle concentre ses recherches sur l'aide sociale, le placement juvénile et l'aide alimentaire dans les pays dits riches.

Sophie Rodari

Sociologue et assistante sociale diplômée, spécialisée en évaluation des politiques publiques, elle est professeure associée à la Haute école de travail social (HESSO/Genève). Ses domaines de recherche principaux sont le rapport à l'argent dans les interventions sociales, les modalités de socialisation professionnelle et la méthodologie professionnelle.

\section{Kim Stroumza}

Docteure en Sciences du langage, elle est professeure associée à la Haute école de travail social (HES-SO/Genève). Elle s'intéresse aux relations entre action, formation et langage. Ses enseignements, ses recherches et ses interventions portent sur les pratiques professionnelles du travail social. Ils s'inscrivent dans le courant de l'analyse de l'activité et visent à modéliser, avec les professionnel.le·s, les savoirfaire que ceux-ci inventent, développent, transforment pour pouvoir répondre à leur mission.

Laurent Wicht

Licencié en Sciences de l'éducation, il est professeur associé à la Haute école de travail social (HES-SO/Genève). Spécialisé dans les questions liées à la jeunesse, ses travaux portent notamment sur les processus de marginalisation et d'intégration des jeunes et sur l'intervention des travailleuses et travailleurs sociaux. Dans ce cadre, il s'intéresse en particulier au travail social de proximité et au territoire local. 
Enseigner, former, publier au cœur de la cité a été achevé d'imprimer en octobre 2018 